\title{
Duality between Wilson loops and gluon amplitudes
}

\author{
Johannes Henn
}

henn@physik.hu-berlin.de

Humboldt-Universität zu Berlin, Institut für Physik, Newtonstr. 15, 12489 Berlin, Germany

\begin{abstract}
An intriguing new duality between planar MHV gluon amplitudes and light-like Wilson loops in $\mathcal{N}=4$ super Yang-Mills is investigated. We extend previous checks of the duality by performing a two-loop calculation of the rectangular and pentagonal Wilson loop. Furthermore, we derive an all-order broken conformal Ward identity for the Wilson loops and analyse its consequences. Starting from six points, the Ward identity allows for an arbitrary function of conformal invariants to appear in the expression for the Wilson loop. We compute this function at six points and two loops and discuss its implications for the corresponding gluon amplitude. It is found that the duality disagrees with a conjecture for the gluon amplitudes by Bern et al. A recent calculation by Bern et al indeed shows that the latter conjecture breaks down at six gluons and at two loops. By doing a numerical comparison with their results we find that the duality between gluon amplitudes and Wilson loops is preserved. This review is based on the author's PhD thesis and includes developments until May 2008.
\end{abstract}

\section{Contents}

1 Introduction 1

2 (Super-)conformal symmetry 6

2.1 Definition of conformal transformations . . . . . . . . . . . . 7

2.2 Conformal correlation functions . . . . . . . . . . . . . . . . . . . . 9

2.3 Supersymmetric extension of the algebra . . . . . . . . . . . . . . . . 11

2.4 The superconformal field theory $\mathcal{N}=4 \mathrm{SYM} \ldots \ldots \ldots \ldots \ldots \ldots$

2.5 Consequences of conformal symmetry for perturbative calculations . . . . . . . 14

2.6 Conformal four-point integrals . . . . . . . . . . . . . . . 20

3 Gluon amplitudes in $\mathcal{N}=4$ SYM

3.1 Introduction . . . . . . . . . . . . . . . . . . 24

3.2 Dual conformal properties of the four-gluon amplitude . . . . . . . . 34

4 Wilson loops and gluon amplitudes at strong coupling 38

4.1 Prescription for computing scattering amplitudes at strong coupling . . . . . . 38

4.2 Computation of 4-cusp Wilson loop at strong coupling . . . . . . . . . . . . . 39

5 Wilson loops

5.1 Renormalisation properties . . . . . . . . . . . . . . . . 42

5.2 Wilson loops in the AdS/CFT correspondence . . . . . . . . . . . . . 44

5.3 Loop equations . . . . . . . . . . . . . . . . . . . . 45

5.4 Light-like Wilson loops . . . . . . . . . . . . . . . . . . . . 45

\footnotetext{
${ }^{1}$ Based on the author's PhD thesis at the university Lyon 1 (France) and prepared at LAPTH, Annecy-le-Vieux (France).
} 
6 Duality between Wilson loops and gluon amplitudes 50

6.1 IR divergences and their relation to Wilson loops . . . . . . . . . . . . . 50

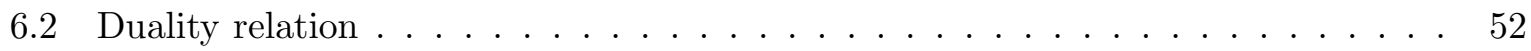

6.3 Duality at one loop . . . . . . . . . . . . . . . 53

6.4 Checks of the duality at two loops and beyond . . . . . . . . . 55

7 Two loop tests of the duality

7.1 Rectangular Wilson loop . . . . . . . . . . . . . . . . 55

7.2 Pentagonal Wilson loop . . . . . . . . . . . . . . . . . 58

7.3 Check of the duality at two loops . . . . . . . . . . . . . 59

8 Conformal symmetry of light-like Wilson loops 59

8.1 Anomalous conformal Ward identities . . . . . . . . . . . . . . 60

8.2 Dilatation Ward identity . . . . . . . . . . . . . . . . . . 61

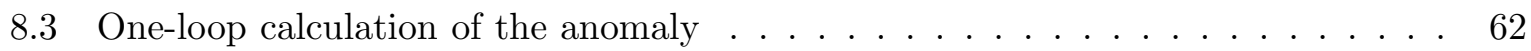

8.4 Structure of the anomaly to all loops . . . . . . . . . . . . . . 63

8.5 Special conformal Ward identity . . . . . . . . . . . . . . 65

8.6 Solution and implications for $F_{n} \ldots \ldots \ldots \ldots \ldots$

9 Hexagon Wilson loop and six-gluon MHV amplitude 68

9.1 Finite part of the hexagon Wilson loop . . . . . . . . . . . . . 68

9.2 Numerical evaluation . . . . . . . . . . . . . . . . . . . . . . . . . 70

9.3 Collinear behaviour . . . . . . . . . . . . . . . . . . 72

9.4 The hexagon Wilson loop versus the six-gluon MHV amplitude . . . . . . . 74

10 Conclusions and outlook $\quad 75$

11 Acknowledgements $\quad 76$

A Alternative proof of $\Phi^{(3)}=\Psi^{(3)}$ using the Mellin-Barnes representation $\mathbf{7 7}$

A.1 Introduction to the Mellin-Barnes technique . . . . . . . . . . . 77

A.2 Example: One-loop integral . . . . . . . . . . . . . . . . 78

A.3 Alternative proof of the magic identity at three loops . . . . . . . . . . . 79

B Two-loop calculation of the rectangular light-like Wilson loop 82

B.1 Computation of individual diagrams . . . . . . . . . . . . 82

B.2 Useful formulae for diagrams with three-gluon vertex . . . . . . . . . . . . . 90

B.3 Basic integrals . . . . . . . . . . . . . . . . . . . 91

B.4 Identities for polylogarithms of related arguments . . . . . . . . . . . . . 91 


\author{
THESE \\ présentée \\ devant l'UNIVERSITE CLAUDE BERNARD - LYON 1 \\ pour l'obtention \\ du DIPLOME DE DOCTORAT \\ (arrêté du 7 août 2006)
}

présentée et soutenue publiquement le 29.09.2008

par

Johannes HENN

\title{
Dualité entre boucles de Wilson et amplitudes de gluons
}

Directeur de thèse : Prof. Emery SOKATCHEV

\begin{tabular}{|c|c|c|c|}
\hline JURY : & $\begin{array}{l}\text { Prof. Costas BACHAS, } \\
\text { Prof. François GIERES, } \\
\text { Prof. Jan PLEFKA, } \\
\text { Prof. Emery SOKATCHEV, } \\
\text { Prof. Kellogg STELLE, }\end{array}$ & $\begin{array}{l}\text { Ecole Normale Supérieure, } \\
\text { Université Lyon 1, } \\
\text { Humboldt Universität Berlin, } \\
\text { Université de Savoie } \\
\text { Imperial College London }\end{array}$ & Rapporteur \\
\hline
\end{tabular}




\section{Introduction}

In the standard model of elementary particles, the strong interactions are described by quantum chromodynamics (QCD). It is a non-Abelian Yang-Mills gauge theory, with the quarks being in the fundamental representation of the gauge group $\mathrm{SU}(3)$. In contrast to quantum electrodynamics, the non-Abelian nature of the gauge group allows the gauge bosons to interact with each other. It is this property which leads to asymptotic freedom and the confinement of quarks. Despite the simplicity and elegance of the QCD Lagrangian, many open problems remain, such as understanding the transition from the high-energy region (short distances), where perturbation theory is valid, to the low-energy region (long distances) of confined quarks.

't Hooft proposed to consider Yang-Mills theories with gauge group $S U(N)$, for $N$ very large, while keeping the 't Hooft coupling $g^{2} N$ (with $g$ being the Yang-Mills coupling constant) fixed [1]. In this way, one may hope to see simplifications for $N$ large, and eventually get insight into QCD by computing terms in an $1 / N$ expansion. The latter resembles that of the genus expansion of string theory. For $N \rightarrow \infty$, non-planar diagrams are suppressed, which is why the 't Hooft limit is also referred to as the planar limit. Hints that simplifications may occur in the 't Hooft limit appeared in [2, 3], where integrable structures were found when studying high-energy scattering in gauge theory. Nevertheless, QCD in the "t Hooft limit is still far from being solved. It seems natural to study supersymmetric Yang-Mills theories, which are much simpler. In particular, the maximally supersymmetric Yang-Mills theory, $\mathcal{N}=4 \mathrm{SYM}$, has many special properties. It was shown long ago that its $\beta$ function vanishes, and hence its coupling constant does not run. It is an interacting superconformal field theory, where the coupling constant is a free parameter. Moreover, through the AdS/CFT correspondence it is expected to be dual to type IIB superstring theory on $A d S_{5} \times S_{5}$ [4]. This is a realisation of 't Hooft's idea of a gauge/string duality in the $N \rightarrow \infty$ limit. The nature of this duality, which relates field theory at strong coupling with string theory at weak coupling, implies that the perturbative series of 'observables' (such as e.g. correlation functions of gauge-invariant operators) in $\mathcal{N}=4$ SYM has to reproduce perturbative string theory results. In order for this to happen, one may suspect that the perturbative expansion of these quantities must have special properties. Indeed, remarkably simple structures have been observed in $\mathcal{N}=4 \mathrm{SYM}$, mainly in two domains: firstly, anomalous dimensions of composite operators and secondly, on-shell $n$-particle scattering amplitudes.

The AdS/CFT correspondence identifies string states with composite, gauge-invariant operators on the gauge theory side. In testing the correspondence, computing scaling dimensions of such operators in $\mathcal{N}=4 \mathrm{SYM}$ plays an important role. The first tests were done for 'protected' operators, whose scaling dimension can be shown to be coupling independent. It therefore equals their classical (tree-level) dimension, which has to agree with the string theory prediction. For generic operators, the scaling dimension depends on the coupling constant, and a comparison with string theory is difficult because of the weak/strong nature of the duality, i.e. it would require summing the complete perturbation series.

Important progress in computing anomalous dimensions in $\mathcal{N}=4 \mathrm{SYM}$ was made with the discovery of integrability in the planar (large $N$ ) limit. In QCD, integrable structures at one loop were observed some time ago [5, 6, 7, 8, 9, 10]. In certain cases, the dilatation operator, which measures the conformal dimension of a given operator, turns out to be described by an integrable spin chain. In $\mathcal{N}=4 \mathrm{SYM}$, the integrability of the complete dilatation operator at one loop was found in [11, 12, 13, 14. Most importantly, in some sectors it was shown to extend to higher loop levels [15, 16, 17, 18, 19, 20, 21, 22, 23, 24, 25]. If this integrability persists to arbitrary loop levels, then one can hope to make contact with strong coupling results. Indeed, 
on the string theory side of the AdS/CFT correspondence, the classical Green-Schwarz superstring action for $A d S_{5} \times S_{5}$, constructed in [26], is integrable [27]. If the integrability survives quantisation [28, 29], it might be possible to identify the integrable structures on both sides of the correspondence.

Integrability, or in certain cases the assumption of integrability, allowed the computation of the anomalous dimensions of various operators in $\mathcal{N}=4 \mathrm{SYM}$. Spectacular progress was made by the all-order conjecture of Beisert, Eden and Staudacher [30. (see also [31, 32, 33, 34, 35]) for the asymptotic Bethe ansatz 2 describing the anomalous dimensions of composite operators in the $S L(2)$ sector of $\mathcal{N}=4 \mathrm{SYM}$. Their proposal leads to an integral equation (BES equation) for the cusp anomalous dimension [36, 37, 38, valid to all orders in the coupling constant. It correctly reproduces the known perturbative values of the cusp anomalous dimension [38, 16, 39, 40] and also agrees with results at strong coupling [41, 42, 43] (for the strong-coupling expansion of the BES equation see [44, 45, 46]).

The second domain where unexpected simplicity was found is that of on-shell gluon scattering. Indeed, the scattering amplitudes turn out to be much simpler than one would expect on general grounds. Even at tree-level, the number of Feynman diagrams contributing to a given amplitude increases factorially with the number of external gluons. Nevertheless, some classes of tree-level amplitudes are known for an arbitrary number of legs. For example, the maximally helicity-violating (MHV) amplitudes are given by very simple one-line expressions [47, 48], and all tree-level amplitudes satisfy recursion relations derived by Britto, Cachazo and Feng (and Witten) [49, 50]. Moreover, tree-level amplitudes have simple properties in twistor space [51].

Furthermore, enormous progress has been achieved to compute loop level amplitudes, mainly using unitarity-based techniques [52, 53], which are inspired by the Cutkosky rules [54]. These have allowed the computation of a large class of one-loop amplitudes, and in the case of $\mathcal{N}=4$ SYM even some two-, three-, and four-loop amplitudes [55, 56, 39, 40]. Anastasiou, Bern, Dixon and Kosower (ABDK) noticed that the four-gluon amplitude at two loops has a remarkable iterative structure [56]. For the infrared divergent terms of the amplitude, such an iteration is expected on general grounds, but a similar iteration was found to hold also for the finite part. Following years of intensive studies of gluon scattering amplitudes [52, 55, 57] and based on the observation of ABDK, the conjecture was put forward by Bern, Dixon and Smirnov [39] that the maximally helicity-violating (MHV) planar gluon amplitudes in $\mathcal{N}=4$ SYM have a remarkably simple all-loop iterative structure. In general, these amplitudes have the following form:

$$
\ln \mathcal{M}_{n}^{(\mathrm{MHV})}=[\mathrm{IR} \text { divergences }]+F_{n}^{(\mathrm{MHV})}\left(p_{1}, \ldots, p_{n} ; a\right)+O(\epsilon) .
$$

Here $\mathcal{M}_{n}^{(\mathrm{MHV})}$ is the colour-ordered planar gluon amplitude, divided by the tree amplitude. The first term on the right-hand side describes the infrared (IR) divergences and the second term is the finite contribution dependent on the gluon momenta $p_{i}$ and on the 't Hooft coupling $a=g^{2} N /\left(8 \pi^{2}\right)$. The structure of IR divergences is well understood in any gauge theory [58, 59, 60, 61, 62, 63, 64, 65, 66, 67, 68, 69, 70, 71, 72, 73. In particular, in theories with a vanishing $\beta$ function like $\mathcal{N}=4 \mathrm{SYM}$, the leading IR singularity in dimensional regularisation is a double pole, whose coefficient is the universal cusp anomalous dimension appearing in many physical processes [38, 74, 75, 16]. Interestingly, the latter is also predicted by integrable models, as mentioned above. The BDS conjecture provides an explicit expression for the finite part, $F_{n}^{(\mathrm{MHV})}=F_{n}^{(\mathrm{BDS})}$, for an arbitrary number $n$ of external gluons, to all orders in the coupling $a$. Remarkably, the dependence of $F_{n}^{(\mathrm{BDS})}$ on the kinematical invariants is described by a function which is coupling independent and, therefore, can be determined at one

\footnotetext{
${ }^{2}$ Asymptotic means that it is valid only for 'long' operators, i.e. valid to $O\left(g^{2 L-2}\right)$, where $L$ is the number of elementary fields constituting the composite operator.
} 
loop. At present, the BDS conjecture has been tested up to three loops for $n=4$ [39] and up to two loops for $n=5$ [76]. The results of this thesis are relevant for the case $n=6$ at two loops.

The aforementioned scattering amplitudes in $\mathcal{N}=4$ have even more interesting features. Drummond, Sokatchev, Smirnov, and the present author found that all integrals appearing in the four-gluon amplitude up to three loops are of a special type [77. In dual momentum variables defined by

$$
p_{i}=x_{i+1}-x_{i}
$$

all integrals can be seen to have broken conformal properties. This is unexpected since this broken dual conformal symmetry is not, at least not in an obvious way, a consequence of the usual (super-)conformal symmetry of $\mathcal{N}=4 \mathrm{SYM}$. This surprising observation was confirmed at four loops [40] and was used as an assumption for constructing the four-gluon amplitude at five loops [78].

In an important recent development in the study of the AdS/CFT correspondence, Alday and Maldacena proposed [79] the strong coupling description of planar gluon scattering amplitudes in $\mathcal{N}=4 \mathrm{SYM}$ and were able to make a direct comparison with the BDS prediction based on weak coupling results for the same amplitudes. According to their proposal, certain planar gluon amplitudes at strong coupling are related to the area of a minimal surface in $\mathrm{AdS}_{5}$ space attached to a specific closed contour $C_{n}$,

$$
\ln \mathcal{M}_{n}=-\frac{\sqrt{g^{2} N}}{2 \pi} A_{\min }\left(C_{n}\right) .
$$

The contour $C_{n}$ is a polygon with light-like edges $\left[x_{i}, x_{i+1}\right]$ defined by the gluon momenta through relation (2), and with the cyclicly condition $x_{n+1} \equiv x_{1}$. Notice that the $x_{i}$, which correspond to the $n$ cusps of the polygon $C_{n}$, are the same dual momentum variables that were introduced previously for discussing the broken conformal properties of integrals appearing in the gluon amplitudes! The prescription of [79] is insensitive to the helicity configuration of the gluon amplitude under consideration.

For $n=4$ the minimal surface $A_{\min }\left(C_{4}\right)$ was found explicitly in [79], by making use of the conformal symmetry of the problem. With the appropriate AdS equivalent of dimensional regularisation, the divergent part of $\ln \mathcal{M}_{4}$ has the expected pole structure, with the coefficient in front of the double pole given by the known strong coupling value of the cusp anomalous dimension. Most importantly, the finite part of $\ln \mathcal{M}_{4}$ is in perfect agreement with $F_{4}^{(\mathrm{BDS})}$ from the BDS ansatz. For $n \geq 5$ the practical evaluation of the solution of the classical string equations turns out to be difficult, but it becomes possible for $n$ large [80. In the limit $n \rightarrow \infty$ the strong coupling prediction for $\ln \mathcal{M}_{n}$ disagrees with the BDS ansatz. This indicates [80] that the BDS conjecture should fail for a sufficiently large number of gluons and/or at sufficiently high loop level.

Alday and Maldacena also pointed out [79 that their prescription (3) is mathematically equivalent to the strong coupling calculation of the expectation value of a Wilson loop $W\left(C_{n}\right)$, defined on the light-like contour $C_{n}$ [81, 82],

$$
W\left(C_{n}\right)=\frac{1}{N}\left\langle 0\left|\operatorname{Tr} \mathrm{P} \exp \left(i g \oint_{C_{n}} d x^{\mu} A_{\mu}\right)\right| 0\right\rangle .
$$

This should not come as a total surprise, since the intimate relationship between the infrared divergences of the scattering of massless particles and the ultraviolet divergences of Wilson loops with cusps is well known in QCD [38, 74, 75]. Inspired by this, Drummond, Korchemsky and 
Sokatchev conjectured that a similar duality relation between planar MHV gluon amplitudes and light-like Wilson loops also exists at weak coupling [83. They illustrated this duality by an explicit one-loop calculation in the simplest case $n=4$. This was later extended to the case of arbitrary $n$ at one loop by Brandhuber, Heslop and Travaglini [84. The duality relation identifies the two objects up to an additive constant and non-planar corrections,

$$
\ln \mathcal{M}_{n}^{(\mathrm{MHV})}=\ln W\left(C_{n}\right)+\mathrm{const}+O(\epsilon, 1 / N) .
$$

This means that upon a specific identification of the regularisation parameters and the kinematical invariants, the infrared divergences of the logarithm of the scattering amplitude $\ln \mathcal{M}_{n}$, match the ultraviolet divergences of the light-like Wilson loop $\ln W\left(C_{n}\right)$, and, most importantly, the finite parts of the two objects also coincide (up to an additive constant and non-planar corrections),

$$
F_{n}^{(\mathrm{MHV})}=F_{n}^{(\mathrm{WL})}+\text { const }+O(1 / N) .
$$

While the former property follows from the known structure of divergences of scattering amplitudes and of Wilson loops in generic gauge theories [38, 85, 74], the property (6) is extremely non-trivial.

In this report we present work in collaboration with Drummond, Korchemsky and Sokatchev that provided further evidence in favour of the duality relation (6). To begin with, we carried out explicit two-loop calculations of $\ln W\left(C_{n}\right)$ for $n=4$ [86] and $n=5$ [87]. Our results are in perfect agreement with the two-loop MHV gluon amplitude calculations [56, 76], and hence with the BDS ansatz for $n=4,5$.

Furthermore, in [86] we argued that we can profit from the (broken) conformal symmetry of the light-like Wilson loops. Due to the presence of a cusp anomaly in the Wilson loops, conformal invariance manifests itself in the form of anomalous Ward identities. In [86] we proposed and in [87] we proved an anomalous conformal Ward identity for the finite part of the Wilson loops, valid to all orders in the coupling constant 3 . It reads

$$
K^{\mu} F_{n}^{(\mathrm{WL})}=\sum_{i=1}^{n}\left(2 x_{i}^{\mu} x_{i}^{\nu} \frac{\partial}{\partial x_{i}^{\nu}}-x_{i}^{2} \frac{\partial}{\partial x_{i \mu}}\right) F_{n}^{(\mathrm{WL})}=\frac{1}{2} \Gamma_{\text {cusp }}(a) \sum_{i=1}^{n} \ln \frac{x_{i, i+2}^{2}}{x_{i-1, i+1}^{2}} x_{i, i+1}^{\mu},
$$

where $x_{i, j}=x_{i}-x_{j}$ and $\Gamma_{\text {cusp }}(a)$ is the (coupling-dependent) cusp anomalous dimension. This identity uniquely fixes the functional form of the finite part of $\ln W\left(C_{n}\right)$ for $n=4$ and $n=5$, up to an additive constant, to agree with the conjectured BDS form for the corresponding MHV gluon amplitudes. For $n \geq 6$, (17) gives partial restrictions on the functional dependence on the kinematical variables. Quite remarkably, the BDS ansatz (1) for the $n$-gluon MHV amplitudes satisfies the conformal Ward identity for arbitrary n [86].

However, for $n \geq 6$ the conformal Ward identity allows $F_{n}^{(\mathrm{WL})}$ to differ from the BDS ansatz $F_{n}^{(\mathrm{BDS})}$ by an arbitrary function of conformal invariants (for $n=6$ there are three such invariants) [86. This result provided a possible explanation of the BDS conjecture for $n=4,5$ (assuming that the MHV amplitudes have the same conformal properties as the Wilson loop), but left the door open for potential deviations from it for $n \geq 6$. To verify whether the BDS conjecture and/or the proposed duality relation (6) still hold for $n=6$ to two loops, it was necessary to perform explicit two-loop calculations of the finite parts of the six-gluon MHV amplitude $F_{6}^{(\mathrm{MHV})}$, and of the hexagon Wilson loop $F_{6}^{(\mathrm{WL})}$.

\footnotetext{
${ }^{3}$ Later, similar Ward identities were also obtained at strong coupling using the AdS/CFT correspondence in Refs. 88, 89 .
} 
By doing an explicit two-loop calculation we were able to derive a (multiple) parameter integral representation for $F_{6}^{(\mathrm{WL})}$, which we evaluated numerically. We found that $F_{6}^{(\mathrm{WL})}$ differs from the BDS ansatz at two loops by a non-trivial function of conformal cross-ratios. We studied this function numerically and found that it was consistent with the collinear limit (of gluon amplitudes) [90. Later, when the results of the two-loop calculation of the six-gluon MHV amplitude became available, we compared the two results numerically and found agreement with the duality relation (6) [91, 92. The BDS ansatz fails at two loops and at $n=6$, but it fails just in such a way as to preserve the duality with Wilson loops! We take this as strong evidence that the duality relation (6) holds for arbitrary $n$ and to an arbitrary number of loops.

We would like to point out that a weaker form of the duality (6) has already been observed in QCD in the special, high-energy (Regge) limit $s \gg-t>0$ for the four-gluon amplitude up to two loops [75]. The same relationship holds in any gauge theory ranging from QCD to $\mathcal{N}=4$ SYM. The essential difference between these theories is that in the former case the duality is only valid in the Regge limit, whereas in the latter case it is exact in general kinematics. We would like to mention that a thorough analysis of the Regge limit of planar multi-gluon amplitudes in $\mathcal{N}=4$ SYM was recently performed in [93, 94] and it provided further evidence that the BDS ansatz needs to be corrected [94]. 
This report is organised as follows:

Sections 2, 6 are mainly introductory. In section 2, we recall some notions of conformal symmetry and its implications for correlation functions of gauge invariant operators in $\mathcal{N}=4 \mathrm{SYM}$. section 3 briefly introduces the reader to gluon scattering amplitudes, focusing on multi-loop results for maximally helicity-violating (MHV) amplitudes and the BDS conjecture. Following that, section 4 summarises the prescription of Alday and Maldacena to compute gluon scattering amplitudes at strong coupling. This will lead us to consider Wilson loops, and after introducing them in section 5 and summarising their renormalisation properties, we discuss in section 6 the main point of study of this thesis: the duality between gluon amplitudes and Wilson loops. The remaining sections present original work by the author and collaborators. In particular sections 2.6 and 3.2 are based on [77, and sections 7, 8, 9] and appendix B] are based on [86, 87, 90, 91, 95].

The only exceptions to the introductory nature of sections 26 are sections 2.6 and 3.2 , where we present original work on off-shell conformal four-point integrals, and their relevance for onshell gluon scattering amplitudes, respectively. We study the known (to four loops) four-gluon scattering amplitude in $\mathcal{N}=4 \mathrm{SYM}$ and find that it is given in terms of 'pseudo-conformal' integrals.

In section 7, we verify the Wilson loop/gluon amplitude duality in the non-trivial cases of four and five points/gluons at two loops by an explicit Feynman graph calculation of the rectangular and pentagonal Wilson loops.

In section 8, we derive all-order Ward identities for the light-like Wilson loops and discuss their consequences.

From this discussion of the Ward identities in section 8 it will become clear that a crucial test of the duality is at two loops and for six points/gluons. We therefore compute the hexagonal Wilson loop at two loops in section 9 and study its properties in the collinear limit (of gluon amplitudes). We present a numerical comparison with recently available results for the six-gluon MHV amplitude at two loops.

In section 10, we present our conclusions.

There are two appendices. Appendix $\mathrm{A}$ contains an alternative proof of an identity between two conformal integrals derived in section 2.6, using the Mellin-Barnes technique. In appendix B we present the previously unpublished details of the calculation of the four-point Wilson loop at two loops.

\section{2 (Super-)conformal symmetry}

Conformal symmetry in quantum field theory was extensively studied beginning from the late 1960's, see e.g. [96, 97] and references therein. The conformal properties of correlation functions were studied. Conformal symmetry has very strong consequences in two dimensions, where the conformal group has an infinite number of generators. In four-dimensional field theories, which are more relevant for particle physics, the conformal group has only 15 parameters, and as a consequence, it is less restrictive than in two dimensions. Moreover, in generic field theories, conformal symmetry is broken by quantum corrections. An example is (massless) QCD. Nevertheless, studying the deviation from conformal invariance can be useful in practice, as discussed in the review 98. The situation is much better in a field theory which is conformally invariant also at the quantum level. As we will see in section 2.4, the maximally supersymmetric 
Yang-Mills theory in four dimensions, $\mathcal{N}=4 \mathrm{SYM}$, which was discovered in [99, 100], has this remarkable property.

\subsection{Definition of conformal transformations}

The presentation of conformal symmetry follows roughly chapter four of the book [101], where the reader can find more details. Let us consider a $d$-dimensional space with flat metric $\eta_{\mu \nu}$ (the treatment for Euclidean and Minkowski space is identical). By definition, conformal transformations leave the metric invariant up to a local rescaling

$$
x^{\mu} \rightarrow x^{\prime \mu}, \quad d x_{\mu} d x^{\mu} \rightarrow \Lambda(x) d x^{\prime}{ }_{\mu} d x^{\prime \mu} .
$$

Geometrically, (8) means that conformal transformations preserve angles. In order to find the most general solution to (8), consider an arbitrary infinitesimal coordinate transformation

$$
x^{\mu} \rightarrow x^{\prime \mu}=x^{\mu}+\epsilon^{\mu}(x) .
$$

It is then easy to show that in $d>2$ dimensions, the most general form of $\epsilon_{\mu}$ compatible with (8) is given by

$$
\epsilon_{\mu}=a_{\mu}+m_{\mu \nu} x^{\nu}+\lambda x_{\mu}+2(b \cdot x) x_{\mu}-b_{\mu} x^{2}, \quad m_{\mu \nu}=-m_{\nu \mu},
$$

where we used the notations $b \cdot x=b^{\nu} x_{\nu}$ and $x^{2}=x^{\nu} x_{\nu}$. The two-dimensional case is special and we will not treat it here, since we are interested in $d=4$. For more information on conformal symmetry in two dimensions, see for example [101. The infinitesimal transformations in (10) corresponding to the parameters $a^{\mu}$ and $m^{\mu \nu}$ are translations and rotations, respectively. Thus the Poincaré group is a subgroup of the conformal group. The transformations corresponding to $\lambda$ and $b^{\nu}$ are dilatations, and special conformal transformations, respectively. Let us define generators for the infinitesimal transformations according to

$$
x^{\prime \rho}=\left(1+i a^{\mu} P_{\mu}+i m^{\mu \nu} M_{\mu \nu}+i \lambda D+i b^{\mu} K_{\mu}\right) x^{\rho} .
$$

By comparing (11) and (10) it is easy to see that the generators of the conformal group are given by

$$
\begin{aligned}
P_{\mu} & =-i \partial_{\mu}, \\
M_{\mu \nu} & =i\left(x_{\mu} \partial_{\nu}-x_{\nu} \partial_{\mu}\right), \\
D & =-i x^{\mu} \partial_{\mu}, \\
K_{\mu} & =-i\left(2 x_{\mu} x^{\nu} \partial_{\nu}-x^{2} \partial_{\mu}\right) .
\end{aligned}
$$

Here we used the shorthand notation $\partial_{\mu}=\frac{\partial}{\partial x^{\mu}}$. From (12) we can see that the conformal group in four dimensions has 15 generators, i.e. four translation generators $P_{\mu}$, six rotations $M_{\mu \nu}$, a dilatation $D$ and four special conformal boosts $K_{\mu}$. In addition to the usual commutation relations of the Poincaré algebra,

$$
\begin{aligned}
& {\left[P_{\mu}, P_{\nu}\right]=0, \quad\left[M_{\mu \nu}, P_{\rho}\right]=i\left(\eta_{\nu \rho} P_{\mu}-\eta_{\mu \rho} P_{\nu}\right),} \\
& {\left[M_{\mu \nu}, M_{\rho \sigma}\right]=i\left(-\eta_{\mu \sigma} M_{\nu \rho}+\eta_{\nu \sigma} M_{\mu \rho}+\eta_{\mu \rho} M_{\nu \sigma}-\eta_{\nu \rho} M_{\mu \sigma}\right),}
\end{aligned}
$$

the generators (12) have the following commutation relations:

$$
\begin{array}{cl}
{\left[D, P_{\mu}\right]=i P_{\mu},} & {\left[K_{\rho}, M_{\mu \nu}\right]=i\left(\eta_{\rho \mu} K_{\nu}-\eta_{\rho \nu} K_{\mu}\right),} \\
{\left[D, K_{\mu}\right]=-i K_{\mu},} & {\left[K_{\mu}, P_{\nu}\right]=2 i\left(\eta_{\mu \nu} D-M_{\mu \nu}\right) .}
\end{array}
$$

Relations (13) and (14) define the conformal algebra. 
Let us now consider finite conformal transformations. For translations $P^{\mu}$, rotations $M^{\mu \nu}$ and dilatations $D$, the form of the finite transformations are easy to find. Furthermore, it is possible to show that the finite transformation corresponding to the infinitesimal conformal boosts is given by

$$
x^{\prime \mu}=\frac{x^{\mu}-b^{\mu} x^{2}}{1-2 b \cdot x+b^{2} x^{2}} .
$$

For practical purposes it is convenient to introduce another finite transformation, the inversion,

$$
I: x^{\mu} \rightarrow \frac{x^{\mu}}{x^{2}}
$$

The special conformal transformation (15) can be obtained by composing an inversion, a translation by $-b^{\mu}$, and another inversion. Because of this, it will often be convenient to check the conformal invariance (or covariance) of a given translation invariant expression by doing inversions (16) rather than the more complicated special conformal transformations (15). In particular, inversions will be useful to us in order to determine the consequences of conformal symmetry on correlation functions. Note that the inversion (16) is an element of the conformal group not connected to the identity. In other words, there is no infinitesimal generator corresponding to (16).

So far we discussed the action of the conformal group on coordinates. In a quantum field theory, one has to define the action of the conformal generators on fields as well. The conformal generators acting on fundamental fields $\phi_{I}(x)$ with conformal weight $\Delta$ and Lorentz indices $I$ are 4

$$
\begin{aligned}
\mathbb{M}^{\mu \nu} \phi_{I} & =\left(x^{\mu} \partial^{\nu}-x^{\nu} \partial^{\mu}\right) \phi_{I}+\left(m^{\mu \nu}\right)_{I}^{J} \phi_{J} \\
\mathbb{D} \phi_{I} & =x \cdot \partial \phi_{I}+\Delta \phi_{I} \\
\mathbb{P}^{\mu} \phi_{I} & =\partial^{\mu} \phi_{I} \\
\mathbb{K}^{\mu} \phi_{I} & =\left(2 x^{\mu} x \cdot \partial-x^{2} \partial^{\mu}\right) \phi_{I}+2 x^{\mu} \Delta \phi_{I}+2 x_{\nu}\left(m^{\mu \nu}\right)_{I}^{J} \phi_{J} .
\end{aligned}
$$

Here $m^{\mu \nu}$ is the generator of spin rotations, e.g., $m^{\mu \nu}=0$ for a scalar field and $\left(m^{\mu \nu}\right)_{\lambda}{ }^{\rho}=$ $g^{\nu \rho} \delta_{\lambda}^{\mu}-g^{\mu \rho} \delta_{\lambda}^{\nu}$ for a gauge field. From (17) one can in principle determine the variation of fields under finite transformations. For example, a scalar conformal field has the transformation property

$$
\phi(x) \rightarrow \phi^{\prime}\left(x^{\prime}\right)=\left|\frac{\partial x^{\prime}}{\partial x}\right|^{-\Delta / d} \phi(x),
$$

where $\Delta$ is the conformal dimension of $\phi(x)$. Here

$$
\left|\frac{\partial x^{\prime}}{\partial x}\right|=\Lambda(x)^{-d / 2}
$$

is the Jacobian of the conformal transformation of coordinates, c.f. (8). Fields obeying (18) are called primary.

As was already said, we will consider conformal symmetry in four dimensions only. Examples of classically conformal field theories are theories which have only dimensionless parameters in the action (i.e. dimensionless coupling constant, no masses). An explicit example is the scalar $\phi^{4}$ model in four dimensions, whose action reads

$$
S=\int d^{4} x\left[\frac{1}{2} \partial^{\mu} \phi(x) \partial_{\mu} \phi(x)+\frac{g}{4 !} \phi^{4}(x)\right] .
$$

\footnotetext{
${ }^{4}$ The generators $\mathbb{G}$ determine the infinitesimal transformations with parameters $\varepsilon: \phi^{\prime}(x)=\phi(x)+\varepsilon \cdot \mathbb{G} \phi(x)$.
} 
Under translations and rotations, the scalar field transforms as $\phi^{\prime}\left(x^{\prime}\right)=\phi(x)$, so that (20) is invariant under Poincaré transformations. Further, one can see that (201) is invariant under dilatations and special conformal transformations, if $\phi(x)$ transforms according to (18) with weight $\Delta=1$. Note that the coupling constant $g$ in (20) is dimensionless. Dimensional parameters in the Lagrangian, as for example $m$ corresponding to a mass term $m^{2} \phi^{2}(x)$, would spoil conformal symmetry.

Let us stress that the conformal invariance of (20) is only valid classically, and it is broken by quantum corrections. The reason for this is that due to ultraviolet divergences, the coupling constant $g$ and the elementary field $\phi$ have to be renormalised. This is done in the usual way by introducing appropriate renormalisation factors $Z_{\phi}, Z_{g}$ [102]. The net result is that the renormalised coupling constant $g$ depends on the renormalisation scale $\mu$, i.e. the $\beta$ function is nonvanishing,

$$
\mu \frac{d g}{d \mu}=\beta(g) \neq 0 .
$$

This is the generic situation in quantum field theory: the conformal symmetry is lost in the quantum theory, and may reappear only at certain fixed points $g_{c}$ where $\beta\left(g_{c}\right)=0$.

\subsection{Conformal correlation functions}

In this section, we briefly review the implications of conformal symmetry for correlation functions. For a comprehensive review of conformal symmetry in quantum field theory, see e.g. [96, 97.

In a conformal field theory one usually considers primary operators because they have simple transformation properties under conformal transformations, see (18). Requiring conformal covariance of these operators under conformal transformations according to (18) restrains the functional form that their correlation functions can have. As we will see, conformal symmetry is most restrictive for two- and three-point functions of scalar primary operators.

\subsubsection{Two-point functions}

Take for example two scalar operators $O_{1}$ and $O_{2}$ possessing conformal dimension $\Delta_{1}$ and $\Delta_{2}$, respectively. Under conformal transformations their two-point function has to transform according to (18), i.e.

$$
\left\langle O_{1}\left(x_{1}\right) O_{2}\left(x_{2}\right)\right\rangle=\left|\frac{\partial x^{\prime}}{\partial x}\right|_{x=x_{1}}^{\Delta_{1} / d}\left|\frac{\partial x^{\prime}}{\partial x}\right|_{x=x_{2}}^{\Delta_{2} / d}\left\langle O_{1}\left(x_{1}^{\prime}\right) O_{2}\left(x_{2}^{\prime}\right)\right\rangle,
$$

where $d$ is the space-time dimension. From invariance under the Poincaré group (i.e. under translations and rotations) we immediately deduce that their two-point function can only depend on the translation and rotation invariant variable $x_{12}^{2}=\left(x_{1}^{\mu}-x_{2}^{\mu}\right)^{2}$, i.e.

$$
\left\langle O_{1}\left(x_{1}\right) O_{2}\left(x_{2}\right)\right\rangle=f\left(x_{12}^{2}\right) .
$$

Requiring covariance under dilatations with conformal weights $\Delta_{1}$ and $\Delta_{2}$ (c.f. equation (18)), respectively, we find

$$
\left\langle O_{1}\left(x_{1}\right) O_{2}\left(x_{2}\right)\right\rangle=C\left[x_{12}^{2}\right]^{-\left(\Delta_{1}+\Delta_{2}\right) / 2},
$$

where $C$ is an arbitrary normalisation constant. Finally, covariance under conformal boosts requires $\Delta_{1}$ to be equal to $\Delta_{2}$ (this is easiest seen by doing an inversion), and therefore we have

$$
\left\langle O_{1}\left(x_{1}\right) O_{2}\left(x_{2}\right)\right\rangle=C\left[x_{12}^{2}\right]^{-\Delta}, \quad \text { for } \Delta_{1}=\Delta_{2} \equiv \Delta, \quad 0 \text { otherwise. }
$$

Thus, the functional form of $\left\langle O_{1}\left(x_{1}\right) O_{2}\left(x_{2}\right)\right\rangle$ is completely fixed, and the only dynamically determined quantities are the overall normalisation $C$ and scaling dimensions $\Delta_{1}$ and $\Delta_{2}$. 


\subsubsection{Three-point functions}

The same reasoning as in the previous section leads to the most general form of the three-point function of scalar operators,

$$
\left\langle O_{1}\left(x_{1}\right) O_{2}\left(x_{2}\right) O_{3}\left(x_{3}\right)\right\rangle=C\left[x_{12}^{2}\right]^{-\left(\Delta_{1}+\Delta_{2}-\Delta_{3}\right) / 2}\left[x_{23}^{2}\right]^{-\left(\Delta_{2}+\Delta_{3}-\Delta_{1}\right) / 2}\left[x_{13}^{2}\right]^{-\left(\Delta_{1}+\Delta_{3}-\Delta_{2}\right) / 2}
$$

We can immediately check that under an inversion (16), (26) has the correct conformal weight $\Delta_{i}$ at points $i=1,2,3$. An example of relation (26) is the well-known star-triangle identity [103].

Three-point functions of operators with spin are also constrained. For example, consider a spin one operator $V^{\mu}$ of conformal dimension $\Delta_{1}=3$ (for example a conserved current). Its three-point function with two scalar fields of conformal dimension $\Delta_{2}=\Delta_{3}=2$ is

$$
\left\langle V^{\mu}\left(x_{1}\right) O\left(x_{2}\right) O\left(x_{3}\right)\right\rangle=\frac{1}{x_{13}^{2} x_{12}^{2} x_{23}^{2}} Y_{1 ; 23}^{\mu},
$$

where the vector

$$
Y_{1 ; 23}^{\mu}=\frac{x_{12}^{\mu}}{x_{12}^{2}}-\frac{x_{13}^{\mu}}{x_{13}^{2}}
$$

is conformally covariant at point 1 and invariant at points 2 and 3 . The generalisation of (27) to tensor fields of arbitrary spin and to arbitrary conformal dimensions is straightforward (see for example [104]). An application to twist-two operators in $\mathcal{N}=4 \mathrm{SYM}$ can be found in [105]. Three-point functions of spin one operators [106] were studied in the context of anomalies in [107.

\subsubsection{Four-point functions}

An interesting new phenomenon occurs starting from four points. It is then possible to write down conformal invariants in the form of cross-ratios, which in general read

$$
\frac{x_{i j}^{2} x_{k l}^{2}}{x_{i k}^{2} x_{j l}^{2}} .
$$

At four points, there are two independent cross-ratios,

$$
u=\frac{x_{12}^{2} x_{34}^{2}}{x_{13}^{2} x_{24}^{2}}, \quad v=\frac{x_{14}^{2} x_{23}^{2}}{x_{13}^{2} x_{24}^{2}} .
$$

Thus a four-point correlation function contains in general an arbitrary function of $u$ and $v$,

$$
\left\langle O_{1}\left(x_{1}\right) O_{2}\left(x_{2}\right) O_{3}\left(x_{3}\right) O_{4}\left(x_{4}\right)\right\rangle=\frac{1}{x_{13}^{2} x_{24}^{2} x_{12}^{2} x_{34}^{2}} f(u, v),
$$

and the prefactor of $f$ on the r.h.s. of (31) carries the overall conformal weight of the external points (we have chosen $\Delta_{1}=\Delta_{2}=\Delta_{3}=\Delta_{4}=1$ for simplicity).

Let us remark that correlation functions of operators with spin are also constrained, see e.g. [108, 109]. As an example, the most general form of the four-point function of two scalars and two spin-one operators is found to be

$$
\begin{aligned}
\left\langle O_{1}\left(x_{1}\right) O_{2}\left(x_{2}\right)\right. & \left.V_{3}^{\mu}\left(x_{3}\right) V_{4}^{\nu}\left(x_{4}\right)\right\rangle=\frac{1}{x_{12}^{2}}\left[I_{34}^{\mu \nu} f_{1}(u, v)+Y_{3 ; 12}^{\mu} Y_{4 ; 12}^{\nu} f_{2}(u, v)\right. \\
+Y_{3 ; 14}^{\mu} Y_{4 ; 12}^{\nu} f_{3}(u, v) & \left.+Y_{3 ; 12}^{\mu} Y_{4 ; 13}^{\nu} f_{4}(u, v)+Y_{3 ; 14}^{\mu} Y_{4 ; 13}^{\nu} f_{5}(u, v)\right] .
\end{aligned}
$$

Here, the conformal tensor

$$
I_{12}^{\mu \nu}=\frac{\eta^{\mu \nu}}{x_{12}^{2}}-2 \frac{x_{12}^{\mu} x_{12}^{\nu}}{x_{12}^{4}}
$$

carries spin 1 and dimension 1 at points one and two. 


\subsection{Supersymmetric extension of the algebra}

One can extend the Poincaré algebra (13) by supplementing it with fermionic generators satisfying the anti-commutation relations (for an introduction to supersymmetry, see e.g. [110, 111])

$$
\left\{Q_{\alpha}, \bar{Q}_{\dot{\alpha}}\right\}=-2 \sigma_{\alpha \dot{\alpha}}^{\mu} P_{\mu} .
$$

The supersymmetry generators $Q_{\alpha}$ and $\bar{Q}_{\dot{\alpha}}$ commute with $P_{\mu}$, and they transform under the representation $(1 / 2,0)$ and $(0,1 / 2)$ of the Lorentz group, respectively. It is possible to give a representation of the Super-Poincaré algebra on a superspace. This space consists, in addition to the usual commuting variables $x^{\mu}$, of two anticommuting spinors $\theta^{\alpha}$ and $\bar{\theta}^{\dot{\alpha}}$. Then, we can define a supersymmetry transformation with anticommuting parameters $\xi^{\alpha}, \bar{\xi}^{\dot{\alpha}}$ by

$$
\delta_{\xi} x_{\mu}=i\left(\xi \sigma_{\mu} \bar{\theta}-\theta \sigma_{\mu} \bar{\xi}\right), \quad \delta_{\xi} \theta=\xi, \quad \delta_{\xi} \bar{\theta}=\bar{\xi} .
$$

From (35) we can see that the infinitesimal supersymmetry generators are defined by

$$
Q_{\alpha}=\frac{\partial}{\partial \theta^{\alpha}}-i\left(\bar{\theta} \sigma^{\mu}\right)_{\alpha} \partial_{\mu}, \quad \bar{Q}_{\dot{\alpha}}=-\frac{\partial}{\partial \bar{\theta}^{\dot{\alpha}}}+i\left(\bar{\theta} \sigma^{\mu}\right)_{\dot{\alpha}} \partial_{\mu} .
$$

They satisfy the anticommutation relation (34).

If one combines supersymmetry generators and conformal generators (note that from commuting them one gets additional generators), one obtains the superconformal group. In section 2.4. we will study a field theory which has an $\mathcal{N}=4$ extended superconformal symmetry, with symmetry algebra $P S U(2,2 \mid 4)$, see e.g. [112] for more details on the algebra.

We remark that superconformal symmetry constrains correlation functions of (superconformal) primary operators in a similar way as discussed in section 2.2 for conformal symmetry. For more details, see e.g. [113].

\subsection{The superconformal field theory $\mathcal{N}=4 \mathrm{SYM}$}

Let us introduce the field theory studied in this thesis. $\mathcal{N}=4 \mathrm{SYM}$ is the maximally supersymmetric Yang-Mills theory in four dimensions.

\subsubsection{Action in components and supersymmetry transformations}

Its action was first found by dimensional reduction of $\mathcal{N}=1 \mathrm{SYM}$ in ten dimensions [100]. The original action in ten dimensions is

$$
\mathcal{L}=\operatorname{Tr}\left(-\frac{1}{4} F_{M N} F^{M N}+i g \frac{1}{2} \bar{\Psi} \Gamma^{N} \mathcal{D}_{N} \Psi\right)
$$

The (trivial) dimensional reduction consists in requiring that the fields in (37) do not depend on six of the ten spacetime dimensions, i.e.

$$
\partial^{4+m} A^{N}=0, \quad \partial^{4+m} \Psi=0, \quad \partial^{4+m} \bar{\Psi}=0, \quad m=1, \ldots 6 .
$$

Then, one splits the ten-dimensional indices $M, N$ up into four- and six-dimensional ones and makes the following definitions. One has to make a choice for the representation of the ten dimensional $\Gamma$ matrices in terms of four and six dimensional $\gamma$ matrices, e.g. (see [112])

$$
\begin{gathered}
\Gamma_{\mu}=\gamma_{\mu} \otimes 1, \quad \text { for } \mu=1, \ldots, 4, \\
\Gamma_{4+m}=\gamma_{5} \otimes \tilde{\Gamma}_{m}, \quad \text { for } m=1, \ldots 6,
\end{gathered}
$$


where $\gamma_{\mu}$ and $\gamma_{5}$ are the standard Dirac matrices in four-dimensional Minkowski space, and $\tilde{\Gamma}_{m}$ are Dirac matrices in a six-dimensional Euclidean space, which can be written as

$$
\tilde{\Gamma}_{m}=\left[\begin{array}{cc}
0 & \tilde{\sigma}_{m} \\
\tilde{\sigma}_{m}^{-1} & 0
\end{array}\right] \text {. }
$$

The scalars are defined as the last six components of the ten dimensional gauge field $A_{N}$ :

$$
\phi_{m} \equiv A_{4+m} \quad \text { for } m=1, \ldots 6 .
$$

Finally, with the help of the six dimensional $\tilde{\sigma}_{m}$ matrices we can define

$$
\phi_{i j}=-\frac{1}{2}\left(\tilde{\sigma}_{m}\right)_{i j} \phi_{m}
$$

This leads to the following form of the four-dimensional action

$$
\begin{aligned}
\mathcal{L}= & \operatorname{Tr}\left(-\frac{1}{4} F_{\mu \nu} F^{\mu \nu}+i \lambda_{i} \sigma^{\mu} \mathcal{D}_{\mu} \bar{\lambda}^{i}+\frac{1}{2} \mathcal{D}_{\mu} \phi_{i j} \mathcal{D}^{\mu} \phi^{i j}\right. \\
& \left.+i g \lambda_{i}\left[\lambda_{j}, \phi^{i j}\right]+i g \bar{\lambda}^{i}\left[\bar{\lambda}^{j}, \phi_{i j}\right]+g^{2} \frac{1}{4}\left[\phi_{i j}, \phi_{k l}\right]\left[\phi^{i j}, \phi^{k l}\right]\right) .
\end{aligned}
$$

The theory contains a gauge field $A^{\mu}$, four complex fermions $\lambda^{\alpha i}(i=1,2,3,4)$, and six real scalars $\phi^{i j}=-\phi^{j i}$. All fields are in the adjoint representation of the gauge group $S U(N)$.

The action following from (44) can be seen to be invariant under $\mathcal{N}=4$ on-shell supersymmetry transformations (which follow from the $\mathcal{N}=1$ supersymmetry of (37),

$$
\begin{aligned}
\delta A_{\mu} & =i \xi_{i} \sigma_{\mu} \bar{\lambda}^{i}-i \lambda_{i} \sigma_{\mu} \bar{\xi}^{i} \\
\delta \phi_{i j} & =\xi_{i} \lambda_{j}-\xi_{j} \lambda_{i}+\epsilon_{i j k l} \bar{\xi}^{k} \bar{\lambda}^{k} \\
\delta \lambda_{i} & =-\frac{1}{2} i \sigma^{\mu \nu} \xi_{i} F_{\mu \nu}+2 i \sigma^{\mu} \mathcal{D}_{\mu} \phi_{i j} \bar{\xi}^{j}+2 i g\left[\phi_{i j}, \phi^{j k}\right] \xi_{k},
\end{aligned}
$$

where the transformation parameters $\xi$ and $\bar{\xi}$ are chiral and antichiral spinors, respectively. The algebra of the supersymmetry transformations (45) closes up to a gauge transformation and on-shell (i.e. using the equations of motion).

\subsubsection{Finiteness}

The form and relative factors in (44) are completely fixed by $\mathcal{N}=4$ supersymmetry, and for the same reason there is just one coupling constant $g$. A special property of $\mathcal{N}=4 \mathrm{SYM}$ is that its superconformal symmetry is not broken by quantum corrections. The $\beta$ function of $\mathcal{N}=4 \mathrm{SYM}$ was shown to vanish up to three loops by direct calculations [114, 115, 116]. Furthermore, there exist several arguments for the vanishing of the $\beta$ function to all loops [117, 118, 119, 120, 121]. For more details and further references, see the review [112].

Let us remark on different formalisms in which $\mathcal{N}=4 \mathrm{SYM}$ can be studied. Unfortunately, an off-shell $\mathcal{N}=4$ formalism is not available to date, and there are reasons to believe that it does not exist. For this reason one has to resort to less supersymmetric formalisms. For example, one can very well use the action (44), after the usual gauge fixing procedure, for practical calculations. However, it may be advantageous to utilise a manifestly supersymmetric setup, either for calculational convenience, or because of conceptual advantages. For example, one can take a formulation of $\mathcal{N}=4 \mathrm{SYM}$ in terms of $\mathcal{N}=1$ superfields. We will give the necessary definitions in the next section, and present a sample calculation in section 2.5.4. It is 
also possible to use a manifestly $\mathcal{N}=2$ supersymmetric formalism, which employs harmonic superspace [122]. For examples of recent calculations in this setup, see [123, 124]. Further, there is even a $\mathcal{N}=3$ harmonic superspace formalism [122], and its quantisation was considered in [125]. However up to now, no supergraph calculations were carried out in this formalism.

Let us clarify a point that might otherwise lead to confusion. $\mathcal{N}=4 \mathrm{SYM}$ is often referred to as a 'finite' field theory. In a supersymmetric formalism, it is indeed true that propagators and the coupling constant acquire no or only finite corrections in perturbative calculations. On the other hand, in a non-supersymmetric gauge (e.g. the Wess-Zumino gauge), the gauge dependent propagators do get divergent corrections and need to be renormalised by appropriate wavefunction renormalisations, and the same is true for the coupling constant. It is only the $\beta$ function (which is gauge independent) that vanishes.

Furthermore, even in a finite superspace setup, divergences can and usually do arise when one studies composite operators. The latter are traces of products of several operators at the same space-time point. Such operators generically have short distance singularities which have to be regularised. We will see an example in section 2.5 .

\subsubsection{Action of $\mathcal{N}=4 \mathrm{SYM}$ in $\mathcal{N}=1$ superspace}

The field content of $\mathcal{N}=4 \mathrm{SYM}$ can be realised in $\mathcal{N}=1$ superspace by introducing three chiral superfields $\Phi$ and one real gauge superfield $V$. The gauge fixed action in the Feynman gauge, using the conventions of [126] 5 , reads

$$
\begin{aligned}
S=\int d^{4} x d^{2} \theta d^{2} \bar{\theta} & \left\{V^{a} \square V_{a}-\Phi_{I}^{a} \square \Phi_{a}^{\dagger I}-i 2 g f_{a b c} \Phi_{I}^{\dagger a} V^{b} \Phi^{I c}+2 g^{2} f_{a b e} f_{e c d} \Phi_{I}^{\dagger a} V^{b} V^{c} \Phi^{I d}\right. \\
& \left.-\frac{i g \sqrt{2}}{3 !} f^{a b c}\left[\epsilon_{I J K} \Phi_{a}^{I} \Phi_{b}^{J} \Phi_{c}^{K} \delta(\bar{\theta})-\epsilon^{I J K} \Phi_{a I}^{\dagger} \Phi_{b J}^{\dagger} \Phi_{c K}^{\dagger}\right]+\ldots\right\}
\end{aligned}
$$

Here the dots stand for other vertices involving three and more gluons and also ghosts. We do not display them since they will not appear in the one-loop calculation we present later. The $f_{a b c}$ are the structure constants of the gauge group $S U(N)$.

For the study of conformal field theories, it is suitable to write correlators in coordinate (super-)space, rather than in momentum space. The coordinate space propagators following from (46) are

$$
\left\langle\Phi_{I a}^{\dagger}\left(x_{i}, \theta_{i}, \bar{\theta}_{i}\right) \Phi_{b}^{J}\left(x_{j}, \theta_{j}, \bar{\theta}_{j}\right)\right\rangle=-\frac{\delta_{I}^{J} \delta_{a b}}{4 \pi^{2}} e^{i\left(\xi_{i i}+\xi_{j j}-2 \xi_{j i}\right) \cdot \partial_{i}} \frac{1}{x_{i j}^{2}}
$$

and

$$
\left\langle V_{a}\left(x_{i}, \theta_{i}, \bar{\theta}_{i}\right) V_{b}\left(x_{j}, \theta_{j}, \bar{\theta}_{j}\right)\right\rangle=\frac{\delta_{a b}}{8 \pi^{2}} \frac{\delta\left(\theta_{i j}\right) \delta\left(\bar{\theta}_{i j}\right)}{x_{i j}^{2}},
$$

where

$$
x_{i j}=x_{i}-x_{j}, \quad \theta_{i j}=\theta_{i}-\theta_{j}, \quad \xi_{i j}^{\mu}=\theta_{i}^{\alpha} \sigma_{\alpha \dot{\alpha}}^{\mu} \bar{\theta}_{j}^{\dot{\alpha}} .
$$

The Feynman rules can be read off from (46).

\footnotetext{
${ }^{5}$ Except for the definition of the coupling constant, which is $2 g$ here compared to $g$ in $[126$.
} 


\subsection{Consequences of conformal symmetry for perturbative calculations}

In this section, we give a number of examples to show how the abstract conformal correlation functions discussed in section 2.2 are implemented in $\mathcal{N}=4$ SYM. A subtlety arises because the gauge fixing procedure usually (e.g. when using a covariant gauge) breaks the conformal invariance of the action following from (44). Therefore, the conformal predictions for correlation functions of section 2.2 only apply to gauge invariant quantities, in which the gauge dependent variation of the gauge fixing term drops out.

In the next section we give examples of gauge-invariant operators. Then, we introduce the concept of anomalous dimensions and briefly discuss operator mixing in a conformal field theory. Finally, in section 2.5.4 we show how a particular four-point function can be used to compute anomalous dimensions using the operator product expansion.

\subsubsection{Gauge invariant operators}

From what was just said it is clear that in order for conformal properties to be manifest, one should consider gauge independent quantities. There are different possibilities. For example, one can take a trace over several elementary fields multiplied together at the same space-time point. For the gauge group $S U(N)$, the minimal number of fields is two, since $\operatorname{Tr}\left(t_{a}\right)=0$.

Let us give two examples which often appear in the literature on $\mathcal{N}=4 \mathrm{SYM}$. The first,

$$
\mathcal{Q}_{20^{\prime}}^{i j}=\operatorname{Tr}\left(\phi^{i} \phi^{j}-\frac{1}{6} \delta^{i j} \phi_{k} \phi^{k}\right),
$$

is in the representation $\mathbf{2 0}^{\prime}$ of $S U(4)$. It is the lowest component of the energy momentum supermultiplet. By applying supersymmetry transformations, one can generate the conserved currents of $\mathcal{N}=4 \mathrm{SYM}$. For example, in the supersymmetry variation of (50) the $S U(4)$ current of $\mathcal{N}=4$ SYM appears. It reads

$$
J_{A}^{\mu B}=\operatorname{Tr}\left\{\mathcal{D}^{\mu} \phi_{A C} \phi^{C B}-\phi_{A C} \mathcal{D}^{\mu} \phi^{C B}-\frac{i}{2}\left(\bar{\lambda}_{A} \sigma^{\mu} \lambda^{B}-\frac{1}{4} \delta_{A}^{B} \bar{\lambda}_{C} \sigma^{\mu} \lambda^{C}\right)\right\}
$$

and satisfies the conservation equation $\partial_{\mu} J_{A}^{\mu B}=0$. From the point of view of representation theory of $P S U(2,2 \mid 4), \mathcal{Q}_{20^{\prime}}^{i j}$ belongs to a short representation of fixed conformal dimension $\Delta=2$ [127. Thus, its conformal dimension stays at its classical value to all orders in perturbation theory. Furthermore, it is known (see e.g. the review [128]) that two- and three-point function of (50) do not receive quantum corrections.

The second example,

$$
\mathcal{K}=\frac{1}{3} \operatorname{Tr}\left(\phi^{i} \phi_{i}\right),
$$

is a singlet of $S U(4)$. It is the lowest component of the Konishi supermultiplet [129, 130].

Finally, one can also define non-local gauge invariant operators. An example is the Wilson loop

$$
W[C]=\frac{1}{N} \operatorname{Tr} \mathrm{P} \exp \left(i g \oint_{C} d x_{\mu} A^{\mu}\right),
$$

which is a functional of the (closed) contour $C$. Wilson loops defined on particular polygonal contours are in fact one of the main objects of study of this thesis. We will discuss them in much more detail in section 5 . 


\subsubsection{Anomalous dimensions}

In a quantum field theory, the conformal dimension $\Delta$ of an operator depends in general on the coupling constant, and one writes

$$
\Delta(g)=\Delta_{0}+\gamma(g)
$$

where $\Delta_{0}$ is the classical, i.e. tree-level dimension and $\gamma(g)$ is the anomalous dimension of the operator.

Let us start with the operators $\mathcal{Q}_{2 \mathbf{0}^{\prime}}^{i j}$, for which $\Delta(g)=\Delta_{0}$. For practical calculations, it is convenient to employ the $\mathcal{N}=1$ superfield formalism presented in section 2.4.3, Since the $S U$ (4) symmetry of $\mathcal{N}=4 \mathrm{SYM}$ is not manifest in this formalism, we have to decompose $S U(4) \rightarrow S U(3) \times U(1)$. Under this decomposition, $\mathcal{Q}_{20^{\prime}}^{i j}$ gives rise to the following operators (among others):

$$
\mathcal{C}^{I J}=\operatorname{Tr}\left(\Phi^{I} \Phi^{J}\right), \quad \mathcal{C}_{I J}^{\dagger}=\operatorname{Tr}\left(\Phi_{I}^{\dagger} \Phi_{J}^{\dagger}\right) .
$$

Here $I, J=1,2,3$. It is convenient to use them for quantum calculations since they are gauge invariant without the need to include gauge links like $\exp (2 g V)$. Their lowest component is

$$
C^{I J}=\operatorname{Tr}\left(\phi^{I} \phi^{J}\right), \quad C_{I J}^{\dagger}=\operatorname{Tr}\left(\phi_{I}^{\dagger} \phi_{J}^{\dagger}\right)
$$

Let us compute the two-point function of $C^{11}$ and $C_{11}^{\dagger}$ in order to check the absence of perturbative corrections at one loop. We find that the sum of the graphs contributing to the one-loop

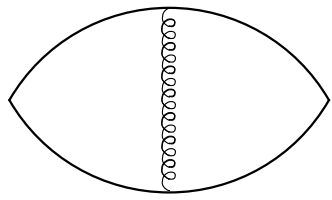

(a)

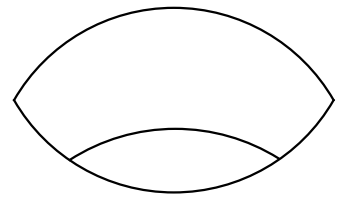

(b)

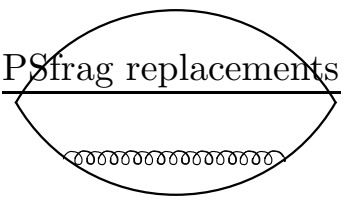

$(c)$

Figure 1: Feynman graphs contributing to $\left\langle C^{11}\left(x_{1}\right) C_{11}^{\dagger}\left(x_{2}\right)\right\rangle$ and $\left\langle\mathcal{K}\left(x_{1}\right) \mathcal{K}\left(x_{2}\right)\right\rangle$ at one loop. Solid lines denote the chiral superfield propagator (47), wiggly lines the gluon superfield propagator (48).

propagator corrections, Fig. 1 (b,c), vanishes identically6. The graph in Fig. 11 (a) leads to an integral that can be shown to vanish for $x_{12} \neq 0$. Thus, up to contact terms, we have

$$
\left\langle C^{11}\left(x_{1}\right) C_{11}^{\dagger}\left(x_{2}\right)\right\rangle=\frac{c}{\left(x_{12}^{2}\right)^{2}}+O\left(g^{4}\right), \quad c=\frac{N^{2}-1}{2(2 \pi)^{4}} .
$$

As we already said, (57) is in fact valid to all orders in the coupling constant. Comparing (57) to (25), one can see that $C^{11}$ has the conformal dimension $\Delta=2$, which is just the sum of the classical dimensions of its constituents $\phi^{1}$.

The second example is the Konishi operator (52). In the $\mathcal{N}=1$ formalism it is given by

$$
\mathcal{K}=\frac{2}{3} \operatorname{tr}\left(e^{-2 g V} \Phi_{I}^{\dagger} e^{2 g V} \Phi^{I}\right)
$$

The exponentials in (58) are needed to make $\mathcal{K}$ gauge invariant. It has the same classical dimension $\Delta_{0}=2$ as $\mathcal{Q}_{\mathbf{2 0}}^{i j}$, but unlike $\mathcal{Q}_{\mathbf{2 0}}^{i j}$, it does get renormalised. Starting from one loop 7 ,

\footnotetext{
${ }^{6}$ This is true in the Feynman gauge.

${ }^{7} \mathrm{By}$ a calculation to $n$ loops, we mean up to order $g^{2 n}$ in the coupling constant. For composite operators, the pictures would suggest a different loop order.
} 


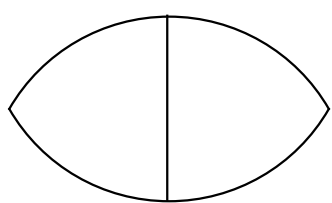

(a)

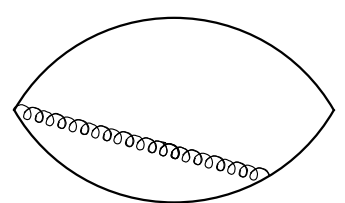

(b)

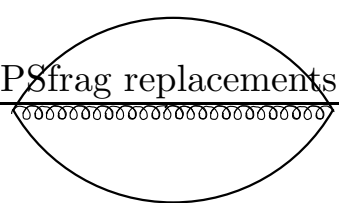

$(c)$

Figure 2: Additional Feynman graphs contributing to $\left\langle\mathcal{K}\left(x_{1}\right) \mathcal{K}\left(x_{2}\right)\right\rangle$ at one loop. Solid lines denote the chiral superfield propagator (47), wiggly lines the gluon superfield propagator (48).

$\mathcal{K}$ has UV divergences typical for composite operators. They come from diagrams (a) and (b) in Figure 2, Evaluating them in dimensional regularisation, one finds (for simplicity, we will compute the lowest, i.e. $\theta=\bar{\theta}=0$, component of $\left\langle\mathcal{K}\left(x_{1}\right) \mathcal{K}\left(x_{2}\right)\right\rangle$ only)

$$
\operatorname{Fig}, 2(a)+\operatorname{Fig}, 2(b)=\frac{1}{\left(x_{12}^{2}\right)^{2-2 \epsilon}} g^{2}\left(x_{12}^{2} \mu^{2}\right)^{\epsilon}\left[\frac{1}{\epsilon} \frac{\left(N^{2}-1\right) N}{(2 \pi)^{6}}+O\left(\epsilon^{0}\right)\right] .
$$

The first factor on the r.h.s. of (59) accounts for the engineering dimension of $\left\langle\mathcal{K}\left(x_{1}\right) \mathcal{K}\left(x_{2}\right)\right\rangle$ in dimensional regularisation, and in addition one gets a factor of $g^{2}\left(x_{12}^{2} \mu\right)^{\epsilon}$ for each loop, with $\mu$ being the dimensional regularisation scale. Furthermore, diagrams of the same topology as those in Fig. 1 contribute, but the only difference is the orientation of some matter lines, and they still vanish for $x_{12} \neq 0$. The graph in Fig. 2(c) leads to a finite contribution.

In dimensional regularisation, one defines renormalised operators by multiplying by a suitable renormalisation factor $Z$ [102]. This means that the renormalised operators $[\mathcal{K}]$ are defined by

$$
[\mathcal{K}]=Z \mathcal{K}, \quad Z=1+\frac{z_{1 ; 1}}{\epsilon} g^{2}+O\left(g^{4}\right) .
$$

The $Z$-factor subtracts the poles in $\epsilon$, so that one is left with a finite result. From (59) we can see that one should take $z_{1 ; 1}=(3 N) /\left(8 \pi^{2}\right)$. Expanding in $\epsilon$, we find for the renormalised operator $[\mathcal{K}]$, to one loop

$$
\left\langle[\mathcal{K}]\left(x_{1}\right)[\mathcal{K}]\left(x_{2}\right)\right\rangle=\frac{N^{2}-1}{(2 \pi)^{4}} \frac{1}{x_{12}^{4}}\left[c\left(g^{2}\right)-g^{2} \frac{N}{4 \pi^{2}} \ln \left(x_{12}^{2} \mu^{2}\right)\right]+O\left(g^{4}\right)+O(\epsilon),
$$

with $c\left(g^{2}\right)=1 / 3+g^{2} N /\left(8 \pi^{2}\right)$. One might think that the appearance of logarithms is surprising in a conformal field theory. The reason is that to a given order in the coupling constant, the expansion of (25) leads to these logarithms. Indeed, one can rewrite (61) in the manifestly conformal form (25),

$$
\left\langle[\mathcal{K}]\left(x_{1}\right)[\mathcal{K}]\left(x_{2}\right)\right\rangle=\frac{N^{2}-1}{(2 \pi)^{4}}\left[c\left(g^{2}\right)\left(x_{12}^{2}\right)^{-\Delta_{\mathcal{K}}}\left(\mu^{2}\right)^{-\gamma \mathcal{K}}\right]+O\left(g^{4}\right)+O(\epsilon),
$$

with the conformal dimension of $\mathcal{K}$ and anomalous dimension $\gamma_{\mathcal{K}}$ at one loop being

$$
\Delta_{\mathcal{K}}\left(g^{2}\right)=2+\gamma_{\mathcal{K}}\left(g^{2}\right), \quad \gamma_{\mathcal{K}}=\frac{g^{2} N}{8 \pi^{2}} 6+O\left(g^{4}\right)
$$

\subsubsection{Operator mixing}

Let us briefly remark on the topic of operator mixing (cf. e.g. [102]) in the context of a conformal field theory [104, 131, 132, 133, 134]. Operator mixing occurs, generally speaking, when there are several operators that have the same quantum numbers. A particular example that often appears in the literature are operators in the $s l(2)$-sector of $\mathcal{N}=4 \mathrm{SYM}$, see e.g. [17, 124]. Of 
these, the twist-two operator: 8 are built from two elementary fields, e.g. $\phi^{1}$ and an arbitrary number $j$ of (covariant) derivatives $\mathcal{D}^{\mu}$. For $j=0$, we already encountered one of these operators in the form of $C^{11}=\left(\phi^{1} \phi^{1}\right)$. For general (even) $j$, they read

$$
\mathcal{O}_{j}=\sum_{k=0}^{j} c_{j k} O_{j k}, \quad O_{j k}=\operatorname{tr}\left(\mathcal{D}^{\left\{\mu_{1}\right.} \ldots \mathcal{D}^{\mu_{k}} \phi^{1} \mathcal{D}^{\mu_{k+1}} \ldots \mathcal{D}^{\left.\mu_{j}\right\}} \phi^{1}\right) .
$$

Here, the curly brackets stand for traceless symmetrisation of the vector indices, so that $\mathcal{O}_{j}$ is in a representation of spin $j$. In a generic situation, there is more than one possible distribution of the derivatives on the two fields $\phi^{1}$, which is reflected by the sum in (64) with a priori arbitrary coefficients $c_{j k}$. As a consequence, the operators $O_{j k}$ for a given $j$ mix under renormalisation.

In a generic quantum field theory, the mixing matrix $c_{j k}$ at order $g^{n}$ can be determined by a calculation at order $g^{(n+2)}$. In a conformal field theory, this mixing problem can be resolved using conformal methods that significantly reduce the complexity of the calculations [104, 131]. We developed these methods further in [135]. As a particular example, we were able to determine the mixing matrix $c_{j k}$ at order $g^{2}$ by evaluating only order $g$ Feynman graphs, compared to a conventional calculation at order $g^{4}$. Similarly, the computation of the anomalous dimensions of the twist two operators in (64) can also be reduced in loop order (by one unit of $g^{2}$ ).

One might object that the resolution of the mixing problem is not relevant, since it is known that mixing matrices are scheme dependent. This scheme dependence, however, is under control and is governed by a renormalisation group equation. Moreover, one can define a conformal scheme, in which the properties of conformal correlation functions are realised (see e.g. the review [98]). In contrast, in a generic scheme, such as for example minimal subtraction, the conformal properties discussed in section 2.2 are in general not present.

The anomalous dimensions of the operators in (64) have received considerable attention recently. They are known up to two loops and conjectured at three loops [136. They are also predicted by a conjectured integrable model [30]. It would be desirable to confirm these conjectures to three loops and beyond. A promising technique to achieve this is the determination of the anomalous dimensions through the operator product expansion (OPE) of conformal four-point functions. We conclude this introductory section with a one-loop example of this technique.

\subsubsection{Conformal four-point functions}

Let us consider the four-point function of $\mathcal{Q}_{\mathbf{2} \mathbf{0}^{\prime}}^{i j}$. We already saw in section 2.5 .2 that $\mathcal{Q}_{20^{\prime}}^{i j}$ does not need to be renormalised. Its conformal dimension is equal to its classical dimension, $\Delta=2$. In this case, from formula (31) we find for the four-point correlator

$$
\left\langle C^{11}\left(x_{1}\right) C^{22}\left(x_{2}\right) C^{\dagger}{ }_{11}\left(x_{3}\right) C^{\dagger}{ }_{22}\left(x_{4}\right)\right\rangle=\frac{1}{x_{12}^{2} x_{34}^{2} x_{13}^{2} x_{24}^{2}} f\left(u, v ; g^{2}\right)
$$

The correlator (65) is known up to two loops in perturbation theory [137, 126]. One reason for being interested in it is that the function $f\left(u, v ; g^{2}\right)$ allows to determine the anomalous dimensions of the twist-two operators (64) via the operator product expansion (OPE). This relation has been worked out in [138, 139].

As was already discussed, the two-point functions of chiral primary operators that could in principle contribute to the disconnected piece of (65) vanish (up to possible contact terms, see e.g. [140] for a discussion). The only nonvanishing Feynman graph contributing to (65) at one

\footnotetext{
${ }^{8}$ The twist of an operator is defined as the difference between its classical dimension and its spin.
} 


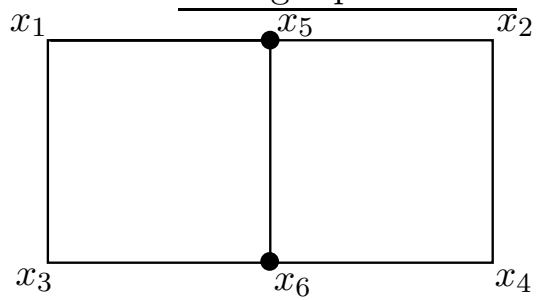

Figure 3: The only connected Feynman graph contributing to $\left\langle C^{11}\left(x_{1}\right) C^{22}\left(x_{2}\right) C^{\dagger}{ }_{11}\left(x_{3}\right) C^{\dagger}{ }_{22}\left(x_{4}\right)\right\rangle$ at one loop. Solid lines denote the chiral superfield propagator (47). The dots represent the (chiral) superspace integrations at points $x_{5}$ and $x_{6}$.

loop is shown in Fig. 3, For (65) we need its lowest component only, i.e. we can set all external $\theta$ 's and $\bar{\theta}$ 's to zero. In that case, using the superfield propagator (47), we obtain

$$
\text { Fig. } 3=\frac{g^{2} N\left(N^{2}-1\right)}{2(2 \pi)^{12}} \frac{1}{x_{12}^{2} x_{34}^{2}} \int d^{4} x_{5} d^{4} x_{6} \int d^{2} \theta_{5} d^{2} \bar{\theta}_{6} \exp \left(i \theta_{5} \partial_{56} \bar{\theta}_{6}\right) \frac{1}{x_{15}^{2} x_{25}^{2} x_{56}^{2} x_{63}^{2} x_{64}^{2}} .
$$

The notation $\partial_{56}$ means that the derivative acts on the term $1 / x_{56}^{2}$ only. The integrals over $\theta_{5}$ and $\bar{\theta}_{6}$ 'see' only the quadratic term in the exponential, i.e.

$$
\int d^{2} \theta_{5} d^{2} \bar{\theta}_{6} \exp \left(i \theta_{5} \partial_{56} \bar{\theta}_{6}\right)=\frac{1}{2} \square_{56},
$$

where $\square=\partial^{\mu} \partial_{\mu}$. Then, we use that

$$
\square \frac{1}{x^{2}}=-4 \pi^{2} \delta^{(4)}(x)
$$

to undo one of the space-time integrals in (66). We arrive at

$$
\text { Fig. } 3=-\frac{g^{2} N\left(N^{2}-1\right)}{2(2 \pi)^{11}} \frac{1}{x_{12}^{2} x_{34}^{2}} h^{(1)}\left(x_{1}, x_{2}, x_{3}, x_{4}\right),
$$

with

$$
h^{(1)}\left(x_{1}, x_{2}, x_{3}, x_{4}\right)=\int \frac{d^{4} x_{5}}{x_{15}^{2} x_{25}^{2} x_{35}^{2} x_{45}^{2}}=\frac{1}{x_{13}^{2} x_{24}^{2}} \Phi^{(1)}(u, v) .
$$

Here $x_{i j}=x_{i}-x_{j}$ and the conformal cross-ratios $u$ and $v$ were defined in (30). The fact that

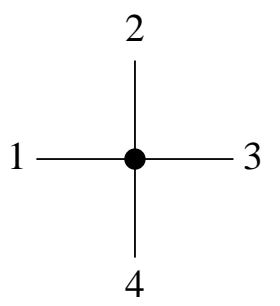

Figure 4: The one-loop ladder integral. Each line represents a propagator with the integration point given by a solid vertex. The reason for the names ladder and box is clearer in the momentum representation of the same integral.

the integral is characterised by a single function of two variables follows from its conformal covariance [141]. Indeed, performing a conformal inversion on all points,

$$
x^{\mu} \longrightarrow \frac{x^{\mu}}{x^{2}} \Longrightarrow x_{i j}^{2} \longrightarrow \frac{x_{i j}^{2}}{x_{i}^{2} x_{j}^{2}}, \quad d^{4} x_{5} \longrightarrow \frac{d^{4} x_{5}}{x_{5}^{8}},
$$


we find that the integral transforms covariantly with weight one at each point,

$$
h^{(1)}\left(x_{1}, x_{2}, x_{3}, x_{4}\right) \longrightarrow x_{1}^{2} x_{2}^{2} x_{3}^{2} x_{4}^{2} h^{(1)}\left(x_{1}, x_{2}, x_{3}, x_{4}\right) .
$$

Since rotation and translation invariance are manifest, we conclude that the integral is given by a conformally covariant combination of propagators multiplied by a function of the conformally invariant cross-ratios (30), in agreement with (65).

The function $\Phi^{(1)}(u, v)$ has been calculated in [142, 143], where it was also shown that the same function appears in a three-point integral. The latter can be obtained from the four-point one by sending one of the points to infinity [141]. We can multiply equation (170) by $x_{13}^{2}$, say, and then take the limit $x_{3} \longrightarrow \infty$. This gives,

$$
h_{3 \mathrm{pt}}^{(1)}\left(x_{1}, x_{2}, x_{4}\right)=\lim _{x_{3} \rightarrow \infty} x_{13}^{2} h^{(1)}\left(x_{1}, x_{2}, x_{3}, x_{4}\right)=\int \frac{d^{4} x_{5}}{x_{15}^{2} x_{25}^{2} x_{45}^{2}}=\frac{1}{x_{24}^{2}} \Phi^{(1)}(\hat{u}, \hat{v}),
$$

where the cross-ratios $u$ and $v$ have become $\hat{u}$ and $\hat{v}$ in the limit,

$$
u \longrightarrow \hat{u}=\frac{x_{12}^{2}}{x_{24}^{2}}, \quad v \longrightarrow \hat{v}=\frac{x_{14}^{2}}{x_{24}^{2}} .
$$

Thus the three-point integral contains the same information as the four-point integral, i.e. the same function of two variables. The reason is that one can use translations and conformal inversion to take the point $x_{3}$ to infinity and the function of the cross-ratios is invariant under these transformations. The explicit formula for $\Phi^{(1)}$ is [144] 9

$$
\Phi^{(1)}(u, v)=\frac{1}{\lambda}\left[2\left(\operatorname{Li}_{2}(-\rho u)+\operatorname{Li}_{2}(-\rho v)\right)+\ln \frac{v}{u} \ln \frac{1+\rho v}{1+\rho u}+\ln (\rho u) \ln (\rho v)+\frac{\pi^{2}}{3}\right],
$$

where

$$
\lambda(u, v)=\sqrt{(1-u-v)^{2}-4 u v}, \quad \rho(u, v)=2(1-u-v+\lambda)^{-1} .
$$

In order to extract the anomalous dimensions of twist two operators, we need $\Phi^{(1)}(u, v)$ in the OPE limit $x_{12} \rightarrow 0$, i.e. $u \rightarrow 0$, only. In this limit, one finds (taking $0<v<1$ for simplicity)

$$
\Phi^{(1)}(u, v)=\frac{1}{1-v}\left[\ln (u) \ln (v)+2 \operatorname{Li}_{2}(1-v)\right]+O(u),
$$

Using the results of [139], one can deduce from (77) the well-known expression for the one-loop anomalous dimensions of the twist-two operators (64),

$$
\gamma(j)=4 a \sum_{k=1}^{j} \frac{1}{k}+O\left(a^{2}\right), \quad a=\frac{g^{2} N}{8 \pi^{2}} .
$$

One can in principle determine the anomalous dimensions at higher orders in perturbation theory from the four-point function (65). It has been computed to two loops in perturbation theory [137, 126]. It would be very interesting to determine its three-loop, i.e. order $g^{6}$, correction in order to test the conjectured formula [136] for the twist-two anomalous dimensions at three loops. Moreover, one may wonder whether the conjectured integrability of $\mathcal{N}=4 \mathrm{SYM}$ contrains the four-point function itself, beyond the contraints coming from conformal symmetry?

\footnotetext{
${ }^{9}$ Valid for $u, v>0$, see [145] for a discussion of the analytic continuation.
} 


\subsection{Conformal four-point integrals}

In section 2.5.4 we saw that from the four-point function of chiral primary operators (65) one can deduce the anomalous dimensions of the twist-two operators (64). Due to the absence of divergences of the chiral primaries, such four-point functions are finite and do not require any regulator. Because of its conformality, a calculation at a given loop order (e.g. with Feynman diagrams) must eventually yield an expression in terms of (finite) conformal integrals. In [77, we took this as a motivation to study an infinite class of conformal four-point integrals. We found that there are simple identities between integrals corresponding to different diagrams. We describe these 'magic identities' in this section.

At the same time, let us stress that in this report, the interest in the conformal integrals mainly comes from an entirely different motivation. We found that, very surprisingly, conformal integrals also appear in the completely unrelated context of on-shell gluon scattering amplitudes in momentum space. As will be explained in section 3.2, their appearance suggests that the latter have a (broken) conformal symmetry in a dual space defined through the gluon momenta. We hope that the example of (65), which illustrates where conformal integrals normally appear (off-shell, finite, in $x$-space) helps clarifying the difference to the integrals that appear when analysing on-shell gluon scattering amplitudes (which are on-shell, IR-divergent, and defined in momentum space) in section 3.2 .

\subsubsection{Proof of conformality}

We will discuss an infinite class of conformal four-point integrals in four dimension 10 , each of which is essentially described by a function of two variables. We already discussed the simplest example, the one-loop ladder integral (70), in section 2.5.4. It is the first in an infinite series of conformal integrals, the $n$-loop ladder (or scalar box) integrals, which have all been evaluated [144]. In particular the 2-loop ladder integral is given by

$$
h^{(2)}\left(x_{1}, x_{2}, x_{3}, x_{4}\right)=x_{24}^{2} \int \frac{d^{4} x_{5} d^{4} x_{6}}{x_{15}^{2} x_{25}^{2} x_{45}^{2} x_{56}^{2} x_{26}^{2} x_{46}^{2} x_{36}^{2}}=\frac{1}{x_{13}^{2} x_{24}^{2}} \Phi^{(2)}(u, v) .
$$

The prefactor $x_{24}^{2}$ is present to give conformal weight one at each external point.

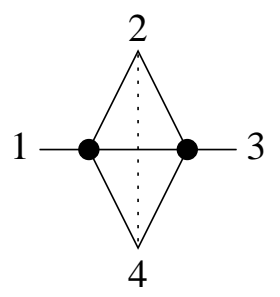

Figure 5: The two-loop ladder integral. The dashed line represents the numerator $x_{24}^{2}$.

Again conformal transformations can be used to justify the appearance of the 2-variable function $\Phi^{(2)}$. The r.h.s. of (79) is invariant under the pairwise swap $x_{1} \longleftrightarrow x_{2}, x_{3} \longleftrightarrow x_{4}$, hence

$$
h^{(2)}\left(x_{2}, x_{1}, x_{4}, x_{3}\right)=h^{(2)}\left(x_{1}, x_{2}, x_{3}, x_{4}\right) .
$$

This symmetry is not immediately evident from the integral. It is its conformal nature which allows this identification. At three loops we consider two conformal integrals, the three-loop

\footnotetext{
${ }^{10}$ In this section we consider and prove identities for Euclidean integrals. The corresponding Minkowskian version of the identities can be obtained through Wick rotation of the integrals. In the Euclidean context we consider integrals with separated external points, $x_{i j} \neq 0$. This is the Euclidean analogue of the off-shell regime, $x_{i j}^{2} \neq 0$, for a Minkowskian integral.
} 


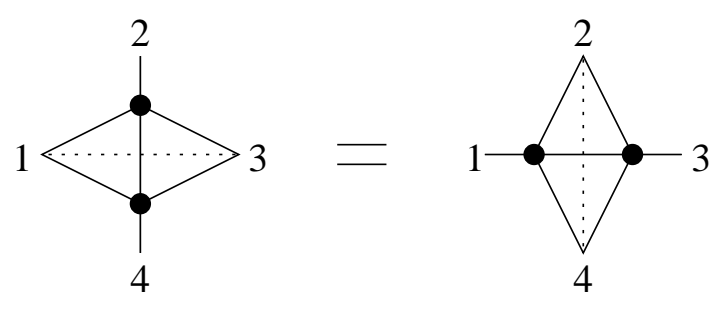

Figure 6: The two-loop turning identity obtained from the pairwise point swap, $x_{1} \longleftrightarrow x_{2}, x_{3} \longleftrightarrow x_{4}$.

ladder,

$$
h^{(3)}\left(x_{1}, x_{2}, x_{3}, x_{4}\right)=x_{24}^{4} \int \frac{d^{4} x_{5} d^{4} x_{6} d^{4} x_{7}}{x_{15}^{2} x_{25}^{2} x_{45}^{2} x_{56}^{2} x_{26}^{2} x_{46}^{2} x_{67}^{2} x_{27}^{2} x_{47}^{2} x_{37}^{2}}=\frac{1}{x_{13}^{2} x_{24}^{2}} \Phi^{(3)}(u, v),
$$

and the so-called 'tennis court' [39],

$$
g^{(3)}\left(x_{1}, x_{2}, x_{3}, x_{4}\right)=x_{24}^{2} \int \frac{x_{35}^{2} d^{4} x_{5} d^{4} x_{6} d^{4} x_{7}}{x_{15}^{2} x_{25}^{2} x_{45}^{2} x_{56}^{2} x_{57}^{2} x_{67}^{2} x_{26}^{2} x_{47}^{2} x_{36}^{2} x_{37}^{2}}=\frac{1}{x_{13}^{2} x_{24}^{2}} \Psi^{(3)}(u, v),
$$

which are shown in Fig. 7. Notice the presence of the numerator $x_{35}^{2}$ in the integrand of the
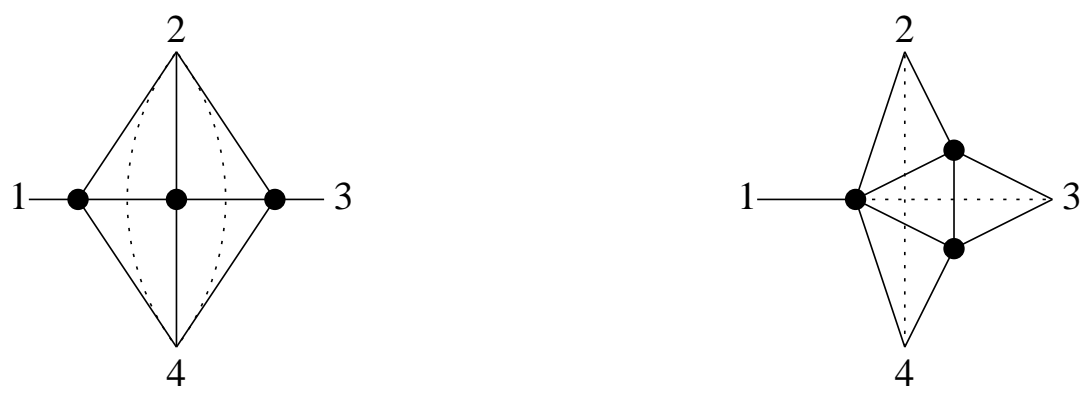

Figure 7: Two examples of three-loop conformal four-point integrals, the three-loop ladder and the 'tennis-court'.

tennis court. It is needed to balance the conformal weight of the five propagators coming out of point 5 .

\subsection{2 'Magic identities' between conformal integrals}

We will show that the three-loop ladder and the tennis court are in fact the same, i.e. we will prove $\Phi^{(3)}=\Psi^{(3)}$. First we shall present a diagrammatic argument. We consider the $n$-loop ladder as being iteratively constructed from the $(n-1)$-loop ladder by integrating against a 'slingshot' (the '0-loop' ladder is a product of free propagators). For example we write the three-loop ladder as

$$
h^{(3)}\left(x_{1}, x_{2}, x_{3}, x_{4}\right)=x_{24}^{2} \int \frac{d^{4} x_{5}}{x_{15}^{2} x_{25}^{2} x_{45}^{2}}\left(x_{24}^{2} \int \frac{d^{4} x_{6} d^{4} x_{7}}{x_{56}^{2} x_{26}^{2} x_{46}^{2} x_{67}^{2} x_{27}^{2} x_{47}^{2} x_{37}^{2}}\right),
$$

where inside the parentheses we recognise the two-loop ladder integral (79). We can then show the equality of the three-loop ladder and the tennis court by using the turning symmetry (80) on the two-loop ladder sub-integral. Then the tennis court integral (82) can be recognised as 

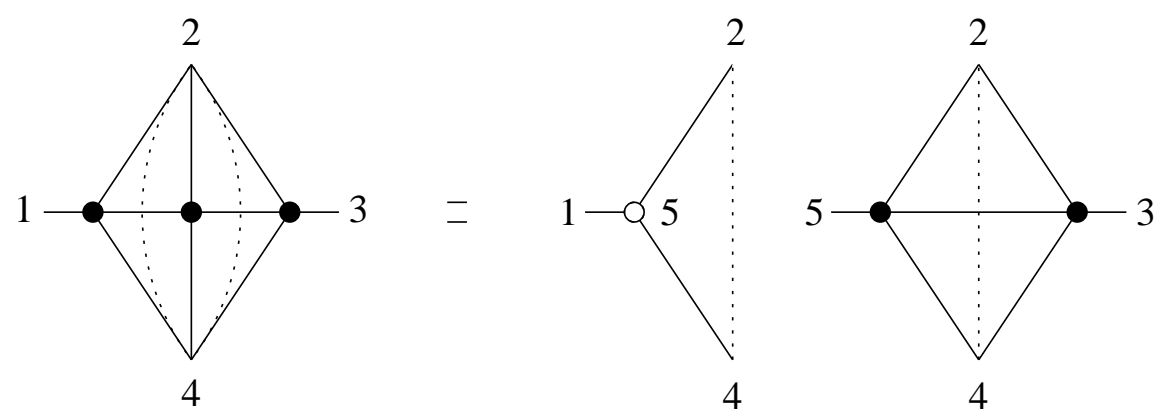

Figure 8: The three-loop ladder expressed as the integral of the two-loop ladder against the 'slingshot'. The empty vertex is the point $x_{5}$ which must be identified with the point $x_{5}$ from the two-loop ladder sub-integral before being integrated over.

the turned two-loop ladder integrated against the slingshot,

$$
\begin{aligned}
h^{(3)}\left(x_{1}, x_{2}, x_{3}, x_{4}\right) & =x_{24}^{2} \int \frac{d^{4} x_{5}}{x_{15}^{2} x_{25}^{2} x_{45}^{2}} h^{(2)}\left(x_{5}, x_{2}, x_{3}, x_{4}\right), \\
& =x_{24}^{2} \int \frac{d^{4} x_{5}}{x_{15}^{2} x_{25}^{2} x_{45}^{2}} h^{(2)}\left(x_{2}, x_{5}, x_{4}, x_{3}\right), \\
& =g^{(3)}\left(x_{1}, x_{2}, x_{3}, x_{4}\right) .
\end{aligned}
$$

This proof can be more easily seen in the diagram (Fig. 9). In using the turning identity (80)
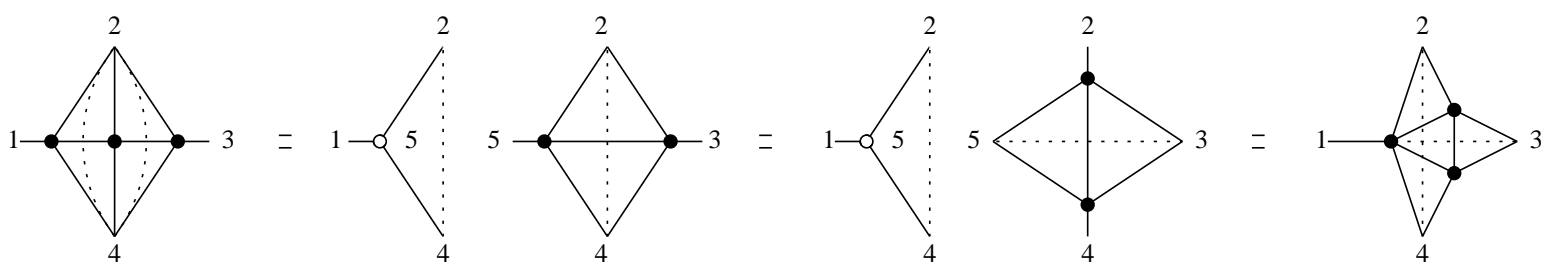

Figure 9: Diagrammatic representation of the proof of equality of the tennis court and the three-loop ladder. The identity follows from the turning identity (80) for the two-loop subintegral.

we have ignored the possibility of contact terms. These could, in principle, spoil the derivation of identities like $\Phi^{(3)}=\Psi^{(3)}$ as the proof (84) involves turning a subintegral. Contact terms could then generate regular terms upon doing one further integration. We now give an argument why this cannot happen for any conformal four-point integral. We again use the example of the 3-loop ladder and tennis court identity.

Consider inserting the $n$-loop subintegral (the 2-loop ladder in this case) into an H-shaped frame with a dashed line across the top, as illustrated below. This generates an $(n+2)$-loop integral which is conformal with weight 1 at each external point (provided the subintegral is conformal with weight 1 at each external point). When inserting the 2 -loop ladder in this way the 4-loop integral one obtains is

$$
\begin{aligned}
f^{(4)}\left(x_{1}, x_{2}, x_{3}, x_{4}\right) & =x_{34}^{2} \int \frac{d^{4} x_{5} d^{4} x_{6}}{x_{15}^{2} x_{45}^{2} x_{56}^{2} x_{26}^{2} x_{36}^{2}} x_{35}^{2} \int \frac{d^{4} x_{7} d^{4} x_{8}}{x_{67}^{2} x_{57}^{2} x_{37}^{3} x_{78}^{2} x_{58}^{2} x_{38}^{2} x_{48}^{2}} \\
& =\frac{1}{x_{13}^{2} x_{24}^{2}} f(u, v) .
\end{aligned}
$$




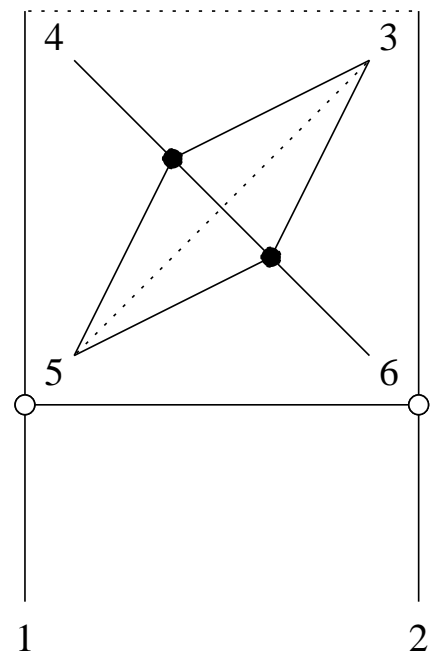

Figure 10: The 2-loop ladder inserted into an H-shaped frame, generating a 4-loop integral.

As usual, the second equality follows from conformality. Now we consider the action of $\square_{1}$ on the above integral using

$$
\square \frac{1}{x^{2}}=-4 \pi^{2} \delta^{(4)}(x)
$$

On the integral one obtains

$$
-4 \pi^{2} \frac{x_{34}^{2} x_{13}^{2}}{x_{14}^{2}} \int \frac{d^{4} x_{6} d^{4} x_{7} d^{4} x_{8}}{x_{26}^{2} x_{16}^{2} x_{36}^{2} x_{67}^{2} x_{17}^{2} x_{37}^{2} x_{78}^{2} x_{18}^{2} x_{38}^{2} x_{48}^{2}}=-\frac{4 \pi^{2} x_{34}^{2}}{x_{13}^{4} x_{14}^{2} x_{24}^{2}} \Phi^{(3)}(u, v) .
$$

On the functional form of (85) one uses the chain rule to derive the action of a differential operator on the function $f$. In this way we find the differential equation,

$$
\frac{x_{23}^{2} x_{34}^{2}}{x_{13}^{6} x_{24}^{4}} \Delta_{u v}^{(2)} f(u, v)=-\frac{\pi^{2} x_{34}^{2}}{x_{13}^{4} x_{14}^{2} x_{24}^{2}} \Phi^{(3)}(u, v) .
$$

The operator $\Delta_{u v}^{(2)}$ is given explicitly by

$$
\Delta_{u v}^{(2)}=u \partial_{u}^{2}+v \partial_{v}^{2}+(u+v-1) \partial_{u} \partial_{v}+2 \partial_{u}+2 \partial_{v} .
$$

Similarly we can act with $\square_{2}$ on the 4-loop integral to obtain the following integral,

$$
-4 \pi^{2} \frac{x_{34}^{2}}{x_{23}^{2}} \int \frac{d^{4} x_{5} d^{4} x_{7} d^{4} x_{8} x_{35}^{2}}{x_{15}^{2} x_{25}^{2} x_{45}^{2} x_{57}^{2} x_{58}^{2} x_{78}^{2} x_{27}^{2} x_{48}^{2} x_{37}^{2} x_{38}^{2}}=-\frac{4 \pi^{2} x_{34}^{2}}{x_{23}^{2} x_{13}^{2} x_{24}^{4}} \Psi^{(3)}(u, v),
$$

and the corresponding differential equation,

$$
\frac{x_{14}^{2} x_{34}^{2}}{x_{24}^{6} x_{13}^{4}} \Delta_{u v}^{(2)} f(u, v)=-\frac{\pi^{2} x_{34}^{2}}{x_{23}^{2} x_{13}^{2} x_{24}^{4}} \Psi^{(3)}(u, v)
$$

From (88191) it follows that $\Phi^{(3)}=\Psi^{(3)}$, the point being that one obtains the same differential operator $\Delta_{s t}^{(2)}$ under the two $\square$ operations. The argument has the obvious generalisation of placing any conformal integral (in any orientation) inside the frame. This argument indirectly shows that the previous argument (84) based on turning the subintegral cannot suffer from contact term contributions. 
The identity we have obtained at three loops is just the first example of an infinite set of identities which all come from the turning symmetry of subintegrals. We generate $(n+1)$-loop integrals by integrating $n$-loop integrals against the slingshot in all possible orientations. The resulting integrals are equal by turning identities of the form (80). At two loops we get just one integral (the two-loop ladder). At three loops we have already seen two equivalent integrals (ladder and tennis court). At four loops we generate two equivalent integrals from the three-loop ladder and three equivalent integrals from the tennis court. Finally, all five four-loop integrals obtained in this way are equivalent by the three-loop identity for the ladder and tennis court (see Fig. 11).

In general it is more common to give the diagrams in the 'momentum' representation (which has nothing to do with the Fourier transform) where we regard the integrations as integrals over loop momenta rather than coordinate space vertices. This representation is neater but the numerators need to be described separately as they do not appear in the diagrams. The momentum-space version of the four generations of integrals from Fig. 11 is given in Fig. 12, The transition between the two notations will be discussed in more detail in section 3. As was already mentioned, integrals related to the ones discussed here will reappear in the analysis of loop corrections to gluon scattering amplitudes in section 3.2,

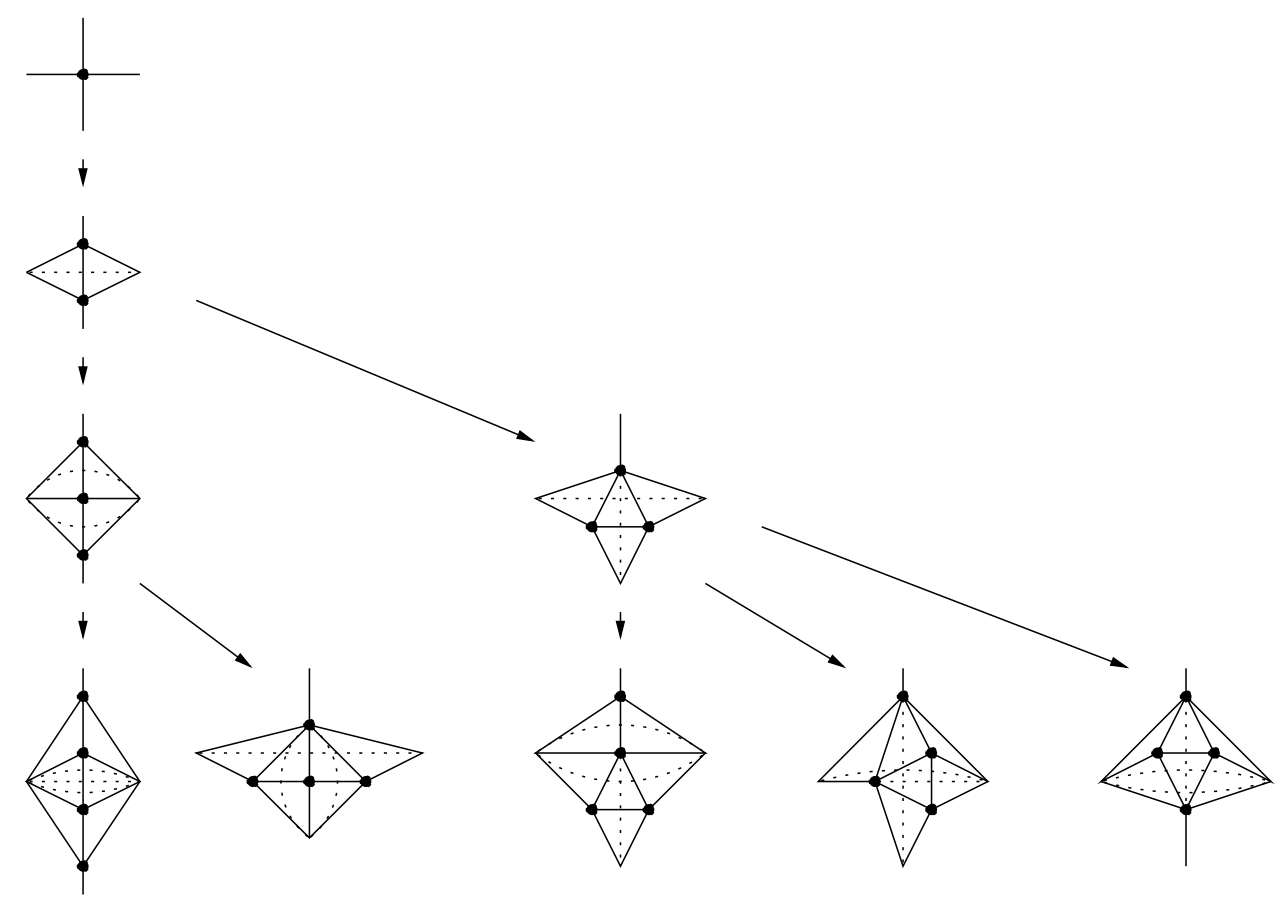

Figure 11: The integrals in a given row are all equivalent. They generate the integrals in the next row by being integrated in all possible orientations against the slingshot attached from above. The ladder series is in the left-most column.

\section{Gluon amplitudes in $\mathcal{N}=4 \mathrm{SYM}$}

\subsection{Introduction}

What are the motivations for computing scattering amplitudes in $\mathcal{N}=4$ SYM? There are several answers to this question. Firstly, being four-dimensional gauge theories, $\mathcal{N}=4 \mathrm{SYM}$ and QCD share some properties, but calculations are generally much harder in the latter. Having efficient calculational methods for computing QCD amplitudes is important for collider physics. The 


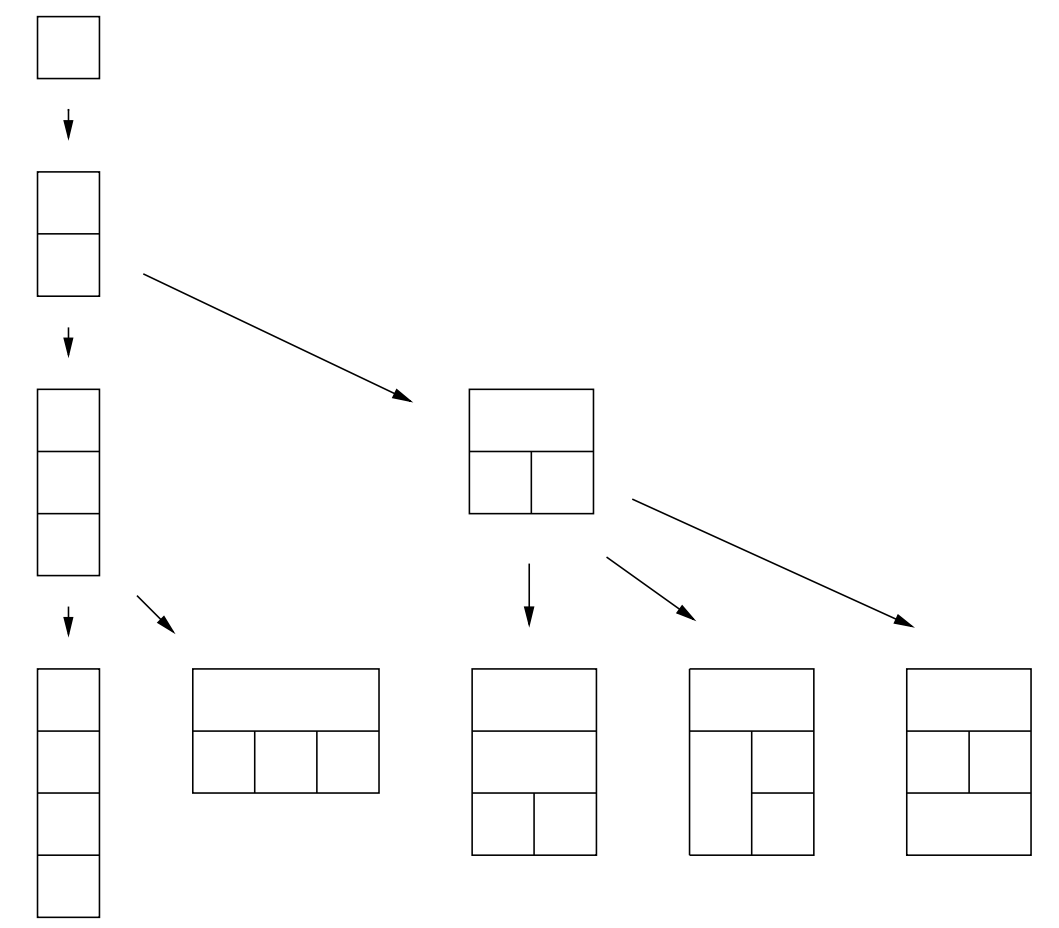

Figure 12: The momentum notation for our integrals up to four loops. The slingshot translates into the top box in each diagram, beneath which are the integrals at one loop lower, arranged in all possible orientations. The ladder series is again in the left-most column.

reason is that in searches for new physics, the theoretical uncertainties for the standard model background processes are often high, in particular in proton-proton collisions [146]. $\mathcal{N}=4$ SYM can be used as a testing ground for new calculational methods. Moreover, a part of QCD amplitudes can be predicted from their $\mathcal{N}=4$ counterparts. For example, tree-level gluon amplitudes in pure Yang-Mills are identical to those in $\mathcal{N}=$ SYM. This follows simply from the Feynman rules. Further, at one loop, gluon amplitudes in pure Yang-Mills can be decomposed in the following way:

$$
A_{g}=(\underbrace{A_{g}+4 A_{f}+3 A_{s}}_{\mathcal{N}=4})-4(\underbrace{A_{f}+A_{s}}_{\mathcal{N}=1})+A_{s} .
$$

Here the abbreviations $g, f, s$ stand for gluon, fermion, and scalar, respectively. They denote the particles circulating in the one-loop diagrams. In this way, a generic one-loop Yang-Mills amplitude can be written in terms of two supersymmetric amplitudes and an amplitude with a scalar circulating in the loop, which is much simpler than the original amplitude.

Another motivation comes from the fact that, as we will discuss in this section, scattering amplitudes contain the cusp anomalous dimension $\Gamma_{\text {cusp }}$, which has received considerable attention over the last years in the study of the AdS/CFT correspondence. Its value is predicted (in principle at any given order) from conjectured integrable models that describe the spectrum of anomalous dimensions in $\mathcal{N}=4 \mathrm{SYM}$. Therefore, knowing $\Gamma_{\text {cusp }}$ to high orders in perturbation theory is important to test and fine-tune these models. The three- and four-loop values of $\Gamma_{\text {cusp }}$ were indeed computed via four-gluon scattering amplitudes.

Gluon scattering amplitudes in $\mathcal{N}=4$ are also studied within the relation between $\mathcal{N}=4$ SYM and $\mathcal{N}=8$ supergravity [147], see [148] for a review. This may give new insights into the ultraviolet properties of $\mathcal{N}=8$ supergravity, see [149] and references therein for a recent discussion. 


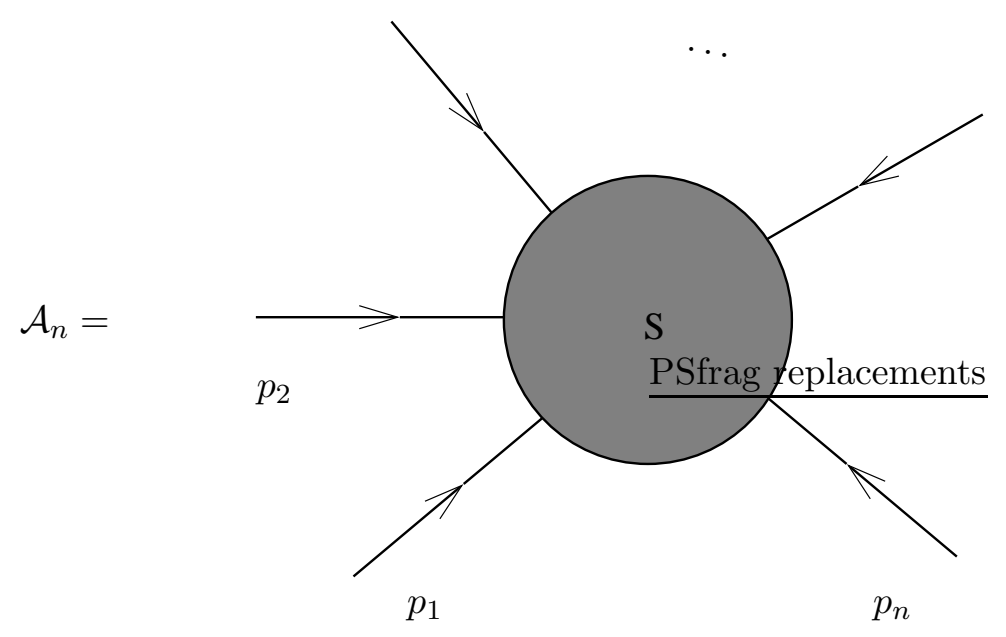

Figure 13: n-gluon scattering

Finally, the scattering amplitudes themselves reveal interesting properties, on which we will focus in this report. As will be described in this section, an iterative structure for the scattering amplitudes in $\mathcal{N}=4 \mathrm{SYM}$ was uncovered by Anastasiou, Bern, Dixon and Kosower (ABDK) [56] and generalised to higher loops by Bern, Dixon and Smirnov (BDS) [39]. In particular, it turns out that their finite part seems to be much simpler than could be expected on general grounds. These discoveries give hope that scattering amplitudes in the large $N$ limit of $\mathcal{N}=4$ SYM may be solvable.

Let us consider an $n$-gluon scattering amplitude in $\mathcal{N}=4 \mathrm{SYM}$, as shown in Fig. 13, All gluons are treated as ingoing, furthermore they are massless and on-shell. They are characterised by their momenta $p_{i}$ (with $p_{i}^{2}=0$ ), their helicity $h_{i}= \pm 1$ and their colour index (in the adjoint representation of the gauge group $S U(N)$ ). There is momentum conservation, i.e. $\sum_{i=1}^{n} p_{i}^{\mu}=0$. Taking the gluon momenta on-shell introduces infrared (IR) divergences that have to be regularised. For this one usually uses a supersymmetry preserving regulator, such as for example dimensional reduction (or a variant of it, the four-dimensional helicity scheme [150, 151]). We will discuss the structure of IR divergences in section 3.1.2.

A generic contribution to an $n$-gluon scattering amplitude can consist of a vast number of terms. For this reason it is very convenient to classify the amplitudes according to their quantum numbers. Let us start by considering the colour structure of the amplitudes. It is clear that the gauge theory factor of a given contribution to the amplitude can be written as a trace or a product of several traces over colour matrices. We are interested in the large $N$ limit, so we neglect multiple traces. It is convenient to define partial amplitudes which correspond to one particular colour structure,

$$
\mathcal{A}_{n}=\operatorname{Tr}\left[T^{a_{1}} T^{a_{2}} \ldots T^{a_{n}}\right] A_{n}^{h_{1}, h_{2}, \ldots, h_{n}}\left(p_{1}, p_{2}, \ldots, p_{n}\right)+\ldots .
$$

The (planar) partial amplitudes $A_{n}^{h_{1}, h_{2}, \ldots, h_{n}}\left(p_{1}, p_{2}, \ldots, p_{n}\right)$ depend on the helicity configuration and on the momenta of the gluons only.

\subsubsection{Helicity structure}

The next simplification comes from classifying the different helicity configurations. In order to do this, let us first introduce the necessary spinor helicity notation. The massless gluon momenta 
$p^{\mu}=\sigma_{\alpha \dot{\alpha}}^{\mu} p^{\alpha \dot{\alpha}}$ can be written as a product of commuting spinors,

$$
p^{\alpha \dot{\alpha}}=\lambda_{p}^{\alpha} \bar{\lambda}_{p}^{\dot{\alpha}}
$$

We also introduce a shorthand notation for spinor products,

$$
\langle p q\rangle=\lambda_{p}^{\alpha} \lambda_{q}^{\beta} \epsilon_{\alpha \beta} \equiv \lambda_{p}^{\alpha} \lambda_{q \alpha}, \quad[p q]=\bar{\lambda}_{p}^{\dot{\alpha}} \bar{\lambda}_{q}^{\dot{\beta}} \epsilon_{\dot{\alpha} \dot{\beta}} \equiv \bar{\lambda}_{p \dot{\alpha}} \bar{\lambda}_{q}^{\dot{\alpha}} .
$$

This allows to write for example a scalar product between two light-like vectors $p^{\mu}$ and $q^{\mu}$ as

$$
\left(p^{\mu}+q^{\mu}\right)^{2}=2 p \cdot q=2\langle p q\rangle[p q] .
$$

The gluons in the partial amplitudes $A_{n}^{h_{1}, h_{2}, \ldots, h_{n}}\left(p_{1}, p_{2}, \ldots, p_{n}\right)$ are projected according to their helicities $h_{i}= \pm 1$ with the following polarisation vectors:

$$
\epsilon_{\mu}^{+}(p)=\left(\sigma_{\mu}\right)_{\alpha \dot{\alpha}} \frac{\lambda_{q}^{\alpha} \bar{\lambda}_{p}^{\dot{\alpha}}}{\sqrt{2}\langle q p\rangle}, \quad \epsilon_{\mu}^{-}(p)=\left(\sigma_{\mu}\right)_{\alpha \dot{\alpha}} \frac{\lambda_{p}^{\alpha} \bar{\lambda}_{q}^{\dot{\alpha}}}{\sqrt{2}[q p]} .
$$

Here $q^{\mu}$ is an arbitrary (up to $q^{\mu} \neq p^{\mu}$ ) reference momentum. A change in $q^{\mu}$ corresponds to a gauge transformation. More information on the spinor helicity notation can be found for example in [152, 153].

In $\mathcal{N}=4 \mathrm{SYM}$, one can define analogous scattering amplitudes where some of the gluons are replaced by fermions or scalars. By requiring invariance of the $S$-matrix under supersymmetry transformations and the invariance of the vacuum state $|0\rangle$, [154, 155], one can derive relations between scattering amplitudes involving different particles and/or helicities. The action of the $\mathcal{N}=4$ supersymmetry generators on gluons $g^{ \pm}$, fermions $f_{i}^{ \pm}$, and scalars $s_{i j}$ is [152]

$$
\begin{aligned}
& {\left[Q_{i}(q, \theta), g^{+}(k)\right]=\theta[k q] f_{i}^{+},} \\
& {\left[Q_{i}(q, \theta), f_{j}^{+}(k)\right]=\theta \delta_{i j}\langle k q\rangle g^{+}+\theta[k q] s_{i j},} \\
& {\left[Q_{i}(q, \theta), s_{j k}(k)\right]=\theta \delta_{i j}\langle k q\rangle f_{k}^{+}-\theta \delta_{i k}\langle k q\rangle f_{j}^{+}+\theta[q k] \epsilon_{i j k l} f_{l}^{-},} \\
& {\left[Q_{i}(q, \theta), f_{j}^{-}(k)\right]=\theta \delta_{i j}[q k] g^{-}+1 / 2 \theta\langle q k\rangle \epsilon_{i j k l} s_{k l},} \\
& {\left[Q_{i}(q, \theta), g^{-}(k)\right]=\theta\langle q k\rangle f_{i}^{-} .}
\end{aligned}
$$

Supersymmetry can be used to derive relations between some amplitudes. For example, acting with $Q_{i}(q, \theta)$ on $\left\langle 0\left|f_{j}^{-}(1) g^{+}(2) \ldots g^{+}(n)\right| 0\right\rangle$, one finds

$$
0=\delta_{i j}\langle q 1\rangle\left\langle 0\left|g^{+}(1) g^{+}(2) \ldots g^{+}(n)\right| 0\right\rangle+[q 1]\left\langle 0\left|s_{i j}(1) g^{+}(2) \ldots g^{+}(n)\right| 0\right\rangle .
$$

Here we have used that amplitudes with two $f^{+}$vanish because of fermion helicity conservation. From (98) it follows 11 that both terms in it vanish. We are mostly interested in gluon scattering, so we note

$$
\left\langle 0\left|g^{+}(1) g^{+}(2) \ldots g^{+}(n)\right| 0\right\rangle=A^{++\ldots+}=0 .
$$

In a similar manner, it can be shown by acting with $Q_{i}(q, \theta)$ on $\left\langle 0\left|g^{-}(1) f_{j}^{+}(2) g^{+}(3) \ldots g^{+}(n)\right| 0\right\rangle$ that

$$
A^{-+\ldots+}=0 .
$$

Furthermore, relations between amplitudes with different particles can be obtained from supersymmetry. Given (99) and (100), the first non-zero pure gluon amplitude has two flipped helicities, and is called maximally helicity-violating (MHV),

$$
A^{(\mathrm{MHV})}=A^{--+\ldots+}, A^{-+-+\ldots+}, \quad \ldots .
$$

\footnotetext{
${ }^{11}$ For $n>3$, because for $n=3$ the prefactor $\langle q 1\rangle$ vanishes for any choice of $q$.
} 
We remark that for four and five gluons, all gluon amplitudes are MHV (or $\overline{\mathrm{MHV}}$, which have all helicities plus except for two, e.g. $A_{5}^{--+++}$), e.g.

$$
\left\{A_{4}^{++--}, \quad A_{4}^{+-+-}, \ldots\right\}, \quad\left\{A_{5}^{+++--}, \quad A_{5}^{+-+--}, \ldots\right\} .
$$

Amplitudes with different helicity ordering are related by cyclicity. It can be shown that in $\mathcal{N}=4 \mathrm{SYM}$, at four and five gluons there is only one independent MHV amplitude.

Amplitudes with more flipped helicities are called NMHV (next to maximally helicityviolating), NNMHV, and so on. NMHV amplitudes (where three helicities are flipped) start appearing at six points, e.g.

$$
A_{6}^{+++---}, \quad A_{6}^{-+--++}, \ldots
$$

MHV amplitudes have the special property that for them, there is only one possible helicity structure, and hence it can be factorised. Using the shorthand notation $\left\langle p_{i} p_{j}\right\rangle=\langle i j\rangle$, we have for $n \geq 4$ [17, 48, up to an inessential normalisation

$$
A_{n}^{(\text {tree })}\left(1^{+}, \ldots, l^{-},(l+1)^{+}, \ldots, m^{-},(m+1)^{+}, \ldots, n^{+}\right)=i \frac{\langle l m\rangle^{4}}{\langle 12\rangle\langle 23\rangle \cdots\langle n 1\rangle},
$$

and all loop corrections are proportional to $A^{\text {(tree) }}$. It is then natural to define the loop corrections to MHV amplitudes by factorising out the tree amplitude, i.e.

$$
A_{n}=A_{n}^{(\text {tree })} M_{n}=A_{n}^{(\text {tree })}\left[1+M_{n}^{(1)}+M_{n}^{(2)}+\ldots\right] .
$$

Here $M_{n}$ is a function of the momentum invariants $p_{i} \cdot p_{j}$ and of the dimensional regulator only. We already said that four- and five-gluon amplitudes are special because all of them are MHV. In contrast, amplitudes with more than two flipped helicities have a more complicated form. Their loop corrections involve in general new helicity structures, and hence they are not proportional to the tree level term. It remains an interesting open question how to extend the duality between Wilson loops and MHV gluon amplitudes discussed in this report to those amplitudes.

\subsubsection{Infrared divergences}

As we already mentioned, the on-shell amplitudes suffer from infrared divergences. In dimensional regularisation, the divergences appear as poles in the regulator $\epsilon$. The structure of these divergences is well understood in any gauge theory $[58,59,60,61,62,63,64,65,66,67,68,69$, 70, 71, 72, 73]. In order to explain the general structure, let us proceed by giving an example. Consider the one-loop corrections to the four-gluon amplitude. They can be written as [155] (with $a=g^{2} N /\left(8 \pi^{2}\right)$ )

$$
A_{4}=A_{4}^{\text {tree }}\left[1-\frac{a}{2} s t I^{(1)}(s, t ; \epsilon)+O\left(a^{2}\right)\right],
$$

where the one-loop scalar box integral $I^{(1)}$ is defined by

$$
I^{(1)}=\overbrace{}^{3}=C \int \frac{d^{4-2 \epsilon} k}{k^{2}\left(k-p_{1}\right)^{2}\left(k-p_{1}-p_{2}\right)^{2}\left(k+p_{4}\right)^{2}},
$$

where $C=\mu^{2 \epsilon} e^{-\epsilon \gamma_{E}}(4 \pi)^{-2+\epsilon}$ and $s=\left(p_{1}+p_{2}\right)^{2}$ and $t=\left(p_{2}+p_{3}\right)^{2}$ are the usual Mandelstam variables. $I^{(1)}$ has infrared divergences coming from two regimes: the soft regime where $k^{2} \sim 0$,

\footnotetext{
${ }^{12}$ One can also define on-shell three-particle scattering for complex momenta.
} 
and the collinear regime where $k^{\mu} \sim p^{\mu}$, with $p^{\mu}$ being one of the on-shell momenta entering the scattering process.

Evaluating (107) one finds, up to terms vanishing as $O(\epsilon)$,

$$
A_{4}=A_{4}^{\text {tree }}\left[1-\frac{a}{\epsilon^{2}}\left(\frac{\mu^{2}}{-s}\right)^{\epsilon}\right]\left[1-\frac{a}{\epsilon^{2}}\left(\frac{\mu^{2}}{-t}\right)^{\epsilon}\right]\left[1+a\left(\frac{1}{2} \ln ^{2} \frac{s}{t}+4 \zeta_{2}\right)\right]+O\left(a^{2}, \epsilon\right) .
$$

We can see from (108) that the loop corrections to $A_{4}$ factorise into divergent pieces, each depending on one kinematical variable only, and a finite part. This factorised structure is known to hold to higher loop orders as well (in the planar case). For non-MHV amplitudes, the only difference compared to (108) is that the finite part in general depends on the helicity structure of the amplitude as well. As we already pointed out, at four points there are only MHV amplitudes, and this problem does not appear. Their general form is known to factorise [64, 71, 156, 157] according to

$$
M_{4}=A_{4} / A_{4}^{\text {tree }}=\operatorname{div}\left(\frac{\mu^{2}}{-s}\right) \operatorname{div}\left(\frac{\mu^{2}}{-t}\right) \operatorname{fin}\left(\frac{s}{t}\right)+O(\epsilon),
$$

where $\operatorname{div}(x)$ contains all poles in $\epsilon$ and $\operatorname{fin}(x)$ is finite as $\epsilon \rightarrow 0$. Notice that the finite part was chosen such that it is independent of the dimensional regularisation parameter $\mu$. The dependence on it is contained in the divergent part and in $O(\epsilon)$ terms only. The divergent factors in (109) are governed by an evolution equation [158, 68, 159, 160, 70. For theories with vanishing $\beta$ function, such as $\mathcal{N}=4 \mathrm{SYM} 13$ the factors containing the divergences take the particularly simple form

$$
\operatorname{div}(x)=\exp \left\{-\frac{1}{2} \sum_{l=1}^{\infty} a^{l} x^{l \epsilon}\left[\frac{\Gamma_{\text {cusp }}^{(l)}}{(l \epsilon)^{2}}+\frac{G^{(l)}}{l \epsilon}\right]\right\}
$$

where

$$
\Gamma_{\text {cusp }}(a)=\sum_{l} a^{l} \Gamma_{\text {cusp }}^{(l)}, \quad G(a)=\sum_{l} a^{l} G_{\text {cusp }}^{(l)} .
$$

Here $\Gamma_{\text {cusp }}$ is the cusp anomalous dimension (of Wilson loops), and $G$ is the scheme-dependent collinear anomalous dimension. Their perturbative expansion in the coupling constant up to two loops is (in the DRED scheme)

$$
\Gamma_{\text {cusp }}(a)=2 a-2 \zeta_{2} a^{2}+O\left(a^{3}\right), \quad G(a)=-\zeta_{3} a^{2}+O\left(a^{3}\right) .
$$

We can see that (108) is indeed of the factorised form expected on general grounds, see formula (109), and the coefficient in front of the double pole agrees with the known expression (112) at one-loop for the cusp anomalous dimension.

By definition [36, 37, 38], the cusp anomalous dimension $\Gamma_{\text {cusp }}(a)$ describes specific ultraviolet divergences of a Wilson loop evaluated over a contour with a cusp. Its appearance in the infrared divergent part of the scattering amplitude (110) is not accidental. It has its roots in the deep relation between scattering amplitudes in gauge theory and Wilson loops evaluated over specific contours in Minkowski space-time, defined by the particle momenta [37, 159, 75]. A distinguishing feature of this contour is that it has cusps. As was found in Refs. [37, 159, 75], the infrared divergences of planar scattering amplitudes are in one-to-one correspondence with the cusp ultraviolet divergences of light-like Wilson loops. It should be mentioned that this relation is not specific to $\mathcal{N}=4 \mathrm{SYM}$ and it holds in any gauge theory, including QCD. We

\footnotetext{
${ }^{13}$ This necessarily implies that a regulator is used where the $\beta$ function is indeed zero
} 
explain this relationship in a one-loop example in section 6.1.

The two-loop expression for $\Gamma_{\text {cusp }}(a)$ in a generic (supersymmetric) Yang-Mills theory was found in Refs. [38, 16. We remark that in $\mathcal{N}=4 \mathrm{SYM}$ theory, $\Gamma_{\text {cusp }}(a)$ is known at weak coupling to four loops [40, and it is conjectured to be governed to all loops by the BES equation 30. At strong coupling, the solution of the latter produces a strong coupling expansion of $\Gamma_{\text {cusp }}(a)$ 44, 45, 46]. The first few terms of this expansion are in agreement with the existing quantum superstring calculation of Refs. [41, 42, 43]. The non-universal collinear anomalous dimension $G(a)$ is known to four loops at weak coupling [39, 161] and there exists a prediction at strong coupling [79].

For planar MHV amplitudes (where the common helicity structure can be factored out), the generalisation of (109) to an arbitrary number $n$ of gluons is

$$
\mathcal{M}_{n}=\ln M_{n}^{(\mathrm{MHV})}=Z_{n}+F_{n}+O(\epsilon),
$$

with

$$
Z_{n}=-\frac{1}{4} \sum_{l=1}^{n} a^{l}\left(\frac{\Gamma_{\text {cusp }}^{(l)}}{(l \epsilon)^{2}}+\frac{G^{(l)}}{l \epsilon}\right) \sum_{i=1}^{n}\left(-\frac{t_{i}^{[2]}}{\mu^{2}}\right)^{-l \epsilon},
$$

where $t_{i}^{[2]} \equiv s_{i, i+1}=\left(p_{i}+p_{i+1}\right)^{2}$ is the invariant mass of two adjacent gluons with indices $i$ and $i+1$, and the periodicity condition $i+n \equiv i$ is tacitly implied. The finite part $F_{n}=$ $F_{n}\left(t_{i}^{[j]}\right)$ is a dimensionless function of the momentum invariants $t_{i}^{[j]}=\left(p_{i}+\ldots p_{i+j-1}\right)^{2}$ of the scattering process 14. From (113) we can see that MHV amplitudes contain two interesting pieces of information: the cusp anomalous dimension $\Gamma_{\text {cusp }}$ in the double pole term, and an $a$ priori arbitrary function of the momentum invariants in the finite part. What makes the latter particularly interesting is that it turns out not to be arbitrary, but seems to have a very simple form, as we will discuss in section 3.1.4. In some sense, the finite part $F_{n}$ is more interesting than $\Gamma_{\text {cusp }}$ because at a given loop order, it is a function (in general of many variables), whereas $\Gamma_{\text {cusp }}^{(l)}$ is merely one number. Finally, note that $G$ is scheme dependent because one can always arbitrarily redefine the dimensional regularisation scale $\mu$. For the same reason $F_{n}$ contains in general a scheme-dependent constant, but its functional form is scheme independent.

\subsubsection{Calculational technique}

The loop corrections to $M_{4}$ can be represented in terms of scalar loop integrals, as for example the scalar box integral $I^{(1)}$ encountered in the previous section. How do these integrals arise? In a standard approach, one would write down all Feynman graphs contributing to a given amplitude, involving gluons, fermions, scalars, and also ghosts. Then, one has to work out the numerator algebra in the integrals corresponding to these graphs. Usually one also has to use integral reduction identities in order to express the amplitude in terms of master integrals [162]. One can think of the scalar integrals as the result of going through this (lengthy) procedure. With increasing loop order (or number of gluons) this approach involves writing down a large number of Feynman graphs and would be very cumbersome if not impossible. For example, even the tree level formula (104) for large $n$ would require writing down an astronomical number of Feynman graphs, since their number grows factorially with $n$.

This is why one often employs a different technique based on the unitarity of the $S$-matrix, see [163] and references therein. The latter, which describes the scattering of particles in a

\footnotetext{
${ }^{14}$ Note that $F_{n}$ does not depend on all $t_{i}^{[j]}$ independently, because (more than four) four-dimensional vectors have to satisfy Gram determinant constraints.
} 


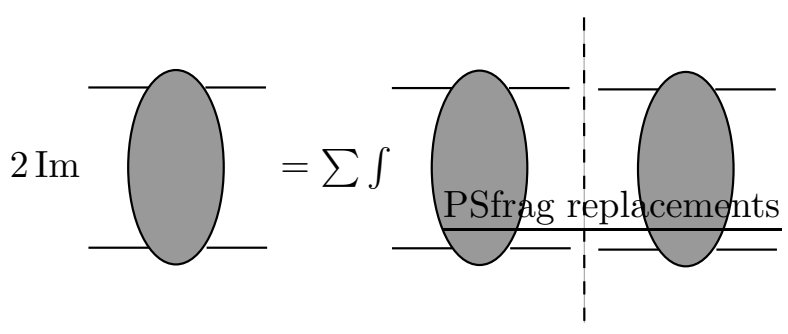

Figure 14: Graphical representation of the unitarity relation $2 \operatorname{Im}(T)=T T^{\dagger}$ for the four-gluon amplitude. The sum is over a complete set of intermediate states.

quantum theory, can be written as

$$
S=1+i T .
$$

Requiring unitarity of the $S$-matrix, $S^{\dagger}=S^{-1}$, one obtains

$$
2 \operatorname{Im}(T)=i\left(T^{\dagger}-T\right)=T T^{\dagger} .
$$

If one inserts a complete set of states between $T$ and $T^{\dagger}$ on the right-hand side of (116), one gets the diagrammatical relation shown in Fig. 14. There, the sum is over all allowed intermediate states and the integral is over their on-shell momenta.

Equation (116) relates objects at different orders in perturbation theory. For example, the imaginary part of a one-loop amplitude on its left-hand side is expressed through tree-level amplitudes on its right-hand side. One can think of the r.h.s. as being a one-loop amplitude that was cut into two tree-level pieces.

In the unitarity based method of Bern et al [52, 53, one does not compute the integral on the right-hand side of (116) directly. Rather, the idea is to reconstruct the amplitudes from their cuts. More precisely, one uses (116) in order to construct the loop integrand of integrals that have the correct behaviour under unitarity cuts. A generic one-loop amplitude can be expressed in terms of master integrals by integral reduction techniques [162]. Therefore it is sufficient to know the coefficients with which the master integrals contribute to the amplitude. Moreover, in supersymmetric field theories, it is known from integral reduction methods, combined with power counting arguments, that only a certain subclass of scalar integrals can appear in the amplitude [53. All of them have either $s$ - or $t$-channel cuts, so they can be detected by the corresponding cuts. Therefore, one-loop amplitudes in $\mathcal{N}=4$ SYM are 'cut-constructible'.

Let us give a one-loop example of the cutting technique, following [153]. Consider the fourgluon amplitude $A_{4}\left(1^{-}, 2^{-}, 3^{+}, 4^{+}\right)$at one loop. From the $s$-channel cut, we have

$$
\begin{aligned}
\left.A_{4}\left(1^{-}, 2^{-}, 3^{+}, 4^{+}\right)\right|_{\mathrm{s}-\text { cut }}= & \sum \int \frac{d^{D} k}{(2 \pi)^{D}} 2 \pi \delta^{(+)}\left(l_{1}^{2}\right) 2 \pi \delta^{(+)}\left(l_{2}^{2}\right) \\
& \times A_{4}^{\text {tree }}\left(-l_{1}, 1^{-}, 2^{-}, l_{2}\right) A_{4}^{\text {tree }}\left(-l_{2}, 3^{+}, 4^{+}, l_{1}\right)
\end{aligned}
$$

Here the cut momenta are $l_{1}=k$ and $l_{2}=k-p_{1}-p_{2}$, with $k$ being the loop momentum. The sum in (117) is over all allowed particles and helicity states for the cut lines. In this example, due to supersymmetric Ward identities, the contribution of scalars and fermions to (117) vanishes. Hence, the only non-vanishing contribution comes from gluon tree amplitudes with the following 
helicities 15

$$
\begin{aligned}
A_{4}^{\text {tree }}\left(-l_{1}^{+}, 1^{-}, 2^{-}, l_{2}^{+}\right) & =i \frac{\langle 12\rangle^{4}}{\left\langle-l_{1} 1\right\rangle\langle 12\rangle\left\langle 2 l_{2}\right\rangle\left\langle l_{2}-l_{1}\right\rangle}, \\
A_{4}^{\text {tree }}\left(-l_{2}^{-}, 3^{+}, 4^{+}, l_{1}^{-}\right) & =i \frac{\left\langle-l_{2} l_{1}\right\rangle^{4}}{\left\langle-l_{2} 3\right\rangle\langle 34\rangle\left\langle 4 l_{1}\right\rangle\left\langle l_{1}-l_{2}\right\rangle} .
\end{aligned}
$$

We now replace the delta functions $2 \pi \delta^{(+)}\left(l_{i}\right)$ by full propagators $i / l_{i}^{2}$, while still using the conditions $l_{i}^{2}=0$ in the numerator. In this way, we construct the integrand of an integral that has the correct behaviour in the $s$-cut, as was explained above.

$$
\begin{aligned}
\left.A_{4}\left(1^{-}, 2^{-}, 3^{+}, 4^{+}\right)\right|_{\text {s-cut }}=\int \frac{d^{D} k}{(2 \pi)^{D}} \frac{1}{l_{1}^{2}} & \frac{\langle 12\rangle^{4}}{l_{2}^{2}} \\
& \times\left.\frac{\left\langle-l_{1} l_{2}\right\rangle^{4}}{\left\langle-l_{1} 1\right\rangle\langle 12\rangle\left\langle 2 l_{2}\right\rangle\left\langle l_{2}-l_{1}\right\rangle}\right|_{\text {s-cut }}
\end{aligned}
$$

By construction, (119) has the correct properties in the $s$-channel. Since we want to arrive at usual loop integrals, we make propagators appear in the denominator by writing e.g.

$$
\frac{1}{\left\langle 2 l_{2}\right\rangle}=-\frac{\left[2 l_{2}\right]}{\left(k-p_{1}\right)^{2}} \text {. }
$$

Using identities like (120), we see that we can factor out the tree amplitude,

$$
\left.A_{4 ; 1}\left(1^{-}, 2^{-}, 3^{+}, 4^{+}\right)\right|_{\mathrm{s}-\mathrm{cut}}=-\left.i A_{4}^{\text {tree }} \int \frac{d^{D} k}{(2 \pi)^{D}} \frac{\left[l_{1} 1\right]\langle 14\rangle\left[4 l_{1}\right]\left\langle l_{1} l_{2}\right\rangle\left[l_{2} 3\right]\langle 32\rangle\left[2 l_{2}\right]\left\langle l_{2} l_{1}\right\rangle}{k^{2}\left(k-p_{1}\right)^{4}\left(k-p_{1}-p_{2}\right)^{2}\left(k+p_{4}\right)^{4}}\right|_{\text {s-cut }} .
$$

After some algebra, the numerator in the integrand can be simplified to $-s t\left(k-p_{1}\right)^{2}\left(k+p_{4}\right)^{2}$, up to a term involving the totally antisymmetric Levi-Civita tensor, which drops out of a four-point amplitude. Thus, the final answer is

$$
\left.A_{4 ; 1}\left(1^{-}, 2^{-}, 3^{+}, 4^{+}\right)\right|_{\mathrm{s}-\text { cut }}=-\left.s t A_{4}^{\text {tree }} \int \frac{d^{D} k}{(2 \pi)^{D}} \frac{1}{k^{2}\left(k-p_{1}\right)^{2}\left(k-p_{1}-p_{2}\right)^{2}\left(k+p_{4}\right)^{2}}\right|_{\mathrm{s}-\text { cut }} .
$$

The $t$-channel cut can be evaluated in a similar manner. There, supersymmetry allows scalars and fermions to contribute as well. Taking all contributions into account, one again finds the scalar box integral, just as in the $s$-channel. By consistency, the coefficients obtained in both channels have to match. Thus, we conclude that in $\mathcal{N}=4 \mathrm{SYM} 16$,

$$
A_{4 ; 1}\left(1^{-}, 2^{-}, 3^{+}, 4^{+}\right)=-s t A_{4}^{\text {tree }} \int \frac{d^{D} k}{(2 \pi)^{D}} \frac{1}{k^{2}\left(k-p_{1}\right)^{2}\left(k-p_{1}-p_{2}\right)^{2}\left(k+p_{4}\right)^{2}},
$$

in agreement with (106).

One-loop amplitudes in supersymmetric theories were shown to be 'cut-constructible' in the above sense. We remark that in non-supersymmetric theories, there are rational terms in the amplitude that cannot be detected by cuts, and have to be determined by other means. This problem can be solved, however, by using $D$-dimensional cuts. The reason is that in $D$ dimensions, the rational terms produce cuts that can be detected. This approach further uses a supersymmetric decomposition of one-loop amplitudes (92). This means that only cuts of scalar

\footnotetext{
${ }^{15}$ Note the change of helicity in $l_{1,2}$ depending on whether the lines are ingoing or outgoing

${ }^{16}$ Strictly speaking, the preceding calculation proves (123) up to terms vanishing as $O(\epsilon)$ only, since we used 4-dimensional cuts. To extend the derivation to $O(\epsilon)$ terms one could use $D$-dimensional cuts. However, in 53 it was argued that (123) is valid to all orders in $\epsilon$ (see also [55]).
} 
loops have to be evaluated in $D$ dimensions. These techniques have allowed the computation of several QCD amplitudes at one loop.

In $\mathcal{N}=4 \mathrm{SYM}$ the cutting techniques were successfully applied to higher loop amplitudes as well. They allowed the computation of amplitudes that would have been practically impossible to determine on the basis of Feynman diagrams. We will summarise the results for MHV amplitudes in $\mathcal{N}=4 \mathrm{SYM}$ in the next section. Finally, we mention another important development, that of 'generalised cuts', where one requires more than two propagators to be on shell, see [164].

\subsubsection{Results of perturbative calculations}

Over the last thirty years, considerable efforts have been undertaken to compute loop corrections to scattering amplitudes in QCD and its supersymmetric extensions, see e.g. the review [57]. Most progress has been made for MHV amplitudes in $\mathcal{N}=4 \mathrm{SYM}$. Up to now, the following MHV amplitudes have been computed in $\mathcal{N}=4$ SYM:

- four gluons at two loops [55, 56] and three loops [39]; the four-gluon amplitude is known in terms of loop integrals at four loops [40, and the integrals were evaluated (numerically) up to the second pole term, which allowed to determine $\Gamma_{\text {cusp }}$ at four loops; a conjecture for the integrals appearing at five loops was put forward in 78

- five gluons to two loops [165, 76]

- six gluons to two loops 92 ]

- $n$ gluons to one loop 52

Similar computations in QCD are much harder and up to now are restricted to lower loop orders.

In [56, Anastasiou, Bern, Dixon and Kosower (ABDK) noticed an interesting iterative structure in the two-loop result for the four-gluon amplitude in $\mathcal{N}=4 \mathrm{SYM}$. Based on [56] and the result of their three-loop calculation, Bern, Dixon and Smirnov (BDS) formulated a conjecture for the $n$-gluon amplitudes in $\mathcal{N}=4 \mathrm{SYM}$ to all orders in the coupling constant [39]. As we already discussed, the divergent part of the scattering amplitudes is well understood, so their conjecture is essentially a statement about the finite part. Their conjecture implies that the finite part $F_{n}$, c.f. (113) has the same functional form as at one loop, $F_{n}^{(1)}$, multiplied by the (coupling-dependent) cusp anomalous dimension,

$$
F_{n}=\frac{1}{2} \Gamma_{\text {cusp }}(a) F_{n}^{(1)}+\text { const } .
$$

For example, in application to the four- and five-gluon amplitudes this means that

$$
\begin{aligned}
& F_{4}=\frac{1}{4} \Gamma_{\text {cusp }}(a)\left[\ln ^{2} \frac{s}{t}+\text { const }\right] \\
& F_{5}=\frac{1}{4} \Gamma_{\text {cusp }}(a)\left[\sum_{i=1}^{5} \ln \frac{s_{i, i+1}}{s_{i+1, i+2}} \ln \frac{s_{i+2, i+3}}{s_{i+3, i+4}}+\text { const }\right]
\end{aligned}
$$

where $s, t$ and $s_{i, i+1}=\left(p_{i}+p_{j}\right)^{2}$ are the momentum invariants of the scattering processes.

Why should the finite part of the amplitudes be so simple? One might speculate that some symmetry could be at the origin of this simplicity. In the next section, we will study the four-gluon amplitude in more detail. We will see that indeed one can observe 'dual' conformal properties of the integrals contributing to it, thus providing hints for a broken conformal symmetry governing the gluon amplitudes. We will see in section 8 that the conjectured duality with 


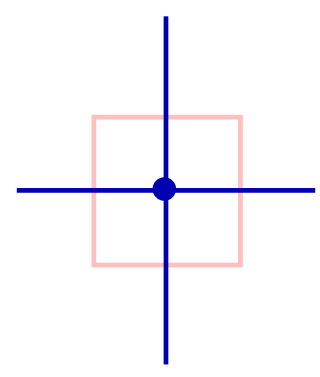

$I^{(1)}$

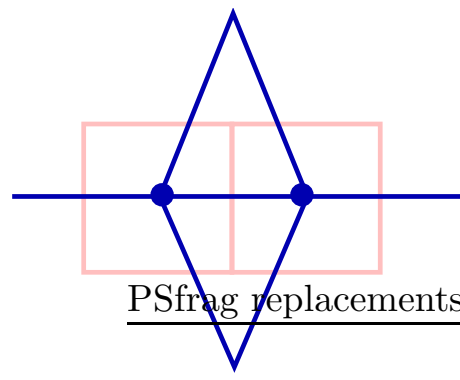

$I^{(2)}$

Figure 15: One-loop box integral $I^{(1)}$ and two loop double box integral $I^{(2)}$ and their dual pictures.

Wilson loops, if true, can explain these observations, and even make them more quantitative, by providing broken conformal Ward identities.

We should stress that very recently, the BDS conjecture was found to fail at six gluons and two loops 92. We will see in section 9 that it is modified in a way predicted by the duality (5) between Wilson loops and gluon amplitudes. The duality, if true, also implies that the BDS conjecture is correct for four and five gluons to all loops (which is consistent with all available data).

\subsection{Dual conformal properties of the four-gluon amplitude}

Let us focus for now on four-gluon amplitudes, where results from high-order calculations in perturbation theory are available. We will see in this section that the integrals contributing to the four-gluon amplitude are all from a set of 'pseudo-conformal' integrals, in the sense that will be defined presently.

Let us come back to the scalar box integral (107). An important observation made in [77] is that it has particular properties in a dual 'coordinate' space, defined by the change of variables

$$
p_{1}=x_{1}-x_{2} \equiv x_{12}, \quad p_{2}=x_{23}, \quad p_{3}=x_{34}, \quad p_{4}=x_{41} .
$$

We stress that (127) is simply a change of variables, and not a Fourier transform. Under the identification (127) and that of $k=x_{15}$, one obtains the expression

$$
I^{(1)}(s, t ; \epsilon)=C \int \frac{d^{4-2 \epsilon} k}{k^{2}\left(k-p_{1}\right)^{2}\left(k-p_{1}-p_{2}\right)^{2}\left(k+p_{4}\right)^{2}}=C \int \frac{d^{4-2 \epsilon} x_{5}}{x_{15}^{2} x_{25}^{2} x_{35}^{2} x_{45}^{2}} .
$$

The integral together with its dual picture is shown in Fig. 15. The point is that in the dual 'coordinate' space defined by (127), the integral $I^{(1)}$ has (broken) conformal properties. Indeed, if it was not for the on-shell conditions $p_{i}^{2}=0$ which lead us to use dimensional regularisation, $I^{(1)}$ would be given by the finite off-shell conformal integral $\Phi^{(1)}$ encountered in section 2.5.4. Recall that $\Phi^{(1)}$ is conformal because under a conformal inversion, the variation of the integral measure is precisely canceled by the variation of the four propagators going to the vertex. In $D=4-2 \epsilon$, we see that the cancelation is no longer exact, and hence the (dual) conformal symmetry is broken. So we conclude that $I^{(1)}$ has broken conformal properties in the dual coordinate space.

In order to avoid confusion with (broken) conformal Ward identities for Wilson loops discussed in later parts of this thesis, we will call integrals of this type 'pseudo-conformal' [40]. 
At this stage one might think that the appearance of the pseudo-conformal integral $I^{(1)}$ is just a one-loop coincidence. However, we will see presently that this pattern continues to hold to higher loops. At two loops,

$$
M_{4}^{(2)}=\frac{1}{4}\left[s^{2} t I^{(2)}(s, t ; \epsilon)+s t^{2} I^{(2)}(t, s ; \epsilon)\right],
$$

one encounters one new integral, the double box integral

$$
I^{(2)}(s, t ; \epsilon)=C^{2} \int \frac{d^{4-2 \epsilon} p d^{4-2 \epsilon} q}{q^{2}\left(p-k_{1}\right)^{2}\left(p-k_{1}-k_{2}\right)^{2}(p+q)^{2} q^{2}\left(q-k_{4}\right)^{2}\left(q-k_{3}-k_{4}\right)^{2}} .
$$

Going to dual coordinates (c.f. Fig. 15), we see that it is the dimensionally continued version of $\Phi^{(2)}$ considered in section 2.6. Notice the dotted line between external points one and three, which is there to make sure that all external points have the same conformal weight. There is an easy way to implement the change of variables (127) by using the following pictorial rule:

- for each loop integration in $p$-space, draw a dot inside the loop, which corresponds to an integration vertex in the dual coordinate space.

- the external points in the dual coordinate space are situated between two consecutive external momenta, denote them by a point.

- draw a straight line between points that are separated by a momentum line.

The numerator lines have to be added by going through the change of variables (127). For pseudo-conformal integrals they are usually easy to find since they have to be chosen such that each integration vertex has conformal weight four, and from the convention that external points have conformal weight one.

We remark that the notion of dual graphs exists for planar graphs only [166]. For this reason it is not clear how to apply this notion to non-planar corrections to gluon scattering amplitudes.

At three loops, the four-gluon amplitude is given by two integrals (see Fig. 16),

$$
M_{4}^{(3)}=\frac{1}{8}\left[s^{3} t I_{4}^{(3) a}(s, t ; \epsilon)+s t^{3} I_{4}^{(3) a}(t, s ; \epsilon)+2 s t^{2} I_{4}^{(3) b}(s, t ; \epsilon)+2 s^{2} t I_{4}^{(3) b}(t, s ; \epsilon)\right],
$$

which are again both 'pseudo-conformal'. The two three-loop integrals appearing in the fourpoint amplitude (131), and depicted in Fig. 16 are

$$
\begin{aligned}
I_{4}^{(3) a}(s, t ; \epsilon)= & C^{3} \int \frac{\mathrm{d}^{4-2 \epsilon} p \mathrm{~d}^{4-2 \epsilon} r \mathrm{~d}^{4-2 \epsilon} q}{p^{2}\left(p-k_{1}\right)^{2}\left(p-k_{1}-k_{2}\right)^{2}} \\
& \times \frac{1}{(p+r)^{2} r^{2}(q-r)^{2}\left(r-k_{3}-k_{4}\right)^{2} q^{2}\left(q-k_{4}\right)^{2}\left(q-k_{3}-k_{4}\right)^{2}},
\end{aligned}
$$

and

$$
\begin{aligned}
I_{4}^{(3) b}(s, t ; \epsilon)= & C^{3} \int \frac{\mathrm{d}^{4-2 \epsilon} p \mathrm{~d}^{4-2 \epsilon} r \mathrm{~d}^{4-2 \epsilon} q(p+r)^{2}}{p^{2} q^{2} r^{2}\left(p-k_{1}\right)^{2}\left(p+r-k_{1}\right)^{2}} \\
& \times \frac{1}{\left(p+r-k_{1}-k_{2}\right)^{2}\left(p+r+k_{4}\right)^{2}\left(q-k_{4}\right)^{2}(r+p+q)^{2}(p+q)^{2}} .
\end{aligned}
$$

The first integral is the triple box integral, which in the dual space corresponds to the dimensionally continued version of $\Phi^{(3)}$. The other three-loop integral, the so-called 'tennis court', 
or rather 'squash court' integral, exhibits a new interesting feature. Its expression contains a numerator $(p+r)^{2}$ (which is not depicted in the momentum space picture), given by $x_{15}^{2}$ in the dual space and depicted by a dotted line. We see that five propagators are connected to integration point five, which is one to many to cancel the conformal weight coming from the transformation of the measure under a conformal inversion. This extra weight is precisely canceled by the presence of the numerator.

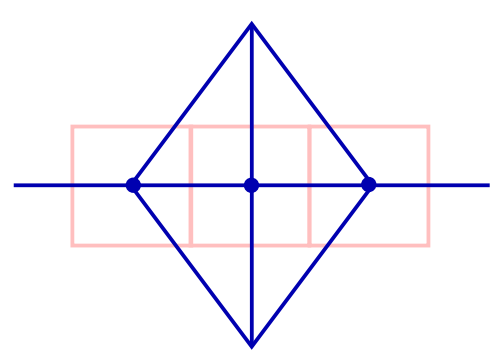

(a)

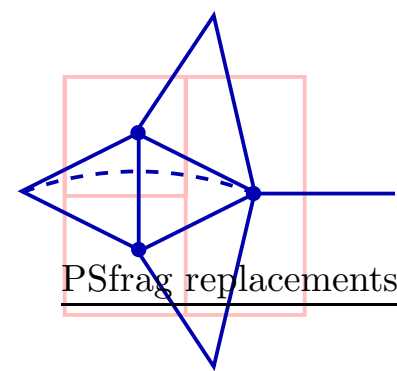

(b)

Figure 16: Three loop ladder or triple box integral $I_{4}^{(3) a}$ and the tennis court integral $I_{4}^{(3) b}$, together with their dual pictures.

At four loops, the number of pseudo-conformal integrals contributing to the amplitude increases compared to three loops, and one finds eight of them [40. They are shown in Figs. 17]18, along with their dual conformal pictures. Let us comment that the integrals shown in Fig. 17] were predicted by the rung rule [39, whereas the integrals shown in Fig. 18 cannot be obtained from it. The rung rule came from the observation that when constructing $(n+1)$-loop integrals from $n$-loop integrals by the unitarity method, the two-particle cuts have an iterative structure, and ( $n+1)$-loop integrals can be obtained by adding a 'rung' to $n$-loop integrals in all possible ways. (see also [167]). It is also interesting to note that in the calculation of [40, two integrals that one might (naively) call pseudo-conformal came with coefficient zero, i.e. they did not appear. A possible explanation for this was found in [83], where it was shown that those latter integrals are not finite in four dimensions for off-shell gluons. It is striking that of all 10 pseudo-conformal integrals, the two integrals that did not appear are both ill-defined in this sense.

Based on the 'conformal hypothesis', i.e. that the expectation that the amplitude should be given by a linear combination of pseudo-conformal integrals, an amplitude in terms of integrals was conjectured at five loops [78. Strikingly, it was found from unitarity cuts that integrals that are ill-defined in the sense discussed in the previous paragraph are absent in the five-gluon amplitude.

We remark that, compared to a generic gauge theory, such as for example QCD, the number of integrals appearing in the final expression for the amplitudes is very small. It seems to be a special property of $\mathcal{N}=4 \mathrm{SYM}$ that the amplitudes can be reduced to such a small set of integrals. In particular, in calculations done so far, it was observed that no diagrams (representing integrals) with triangle or bubble subgraphs appear 17 If true in general, this seems to be a special feature of $\mathcal{N}=4 \mathrm{SYM}$. We remark that if one demands or conjectures that the amplitude should be built from pseudo-conformal integrals only, then triangle and bubble subgraphs are automatically excluded.

Based on [77, 40], in [83] it was conjectured that the four-gluon amplitude is a sum over

\footnotetext{
${ }^{17}$ In the four-loop computation 40 this was used as an assumption.
} 


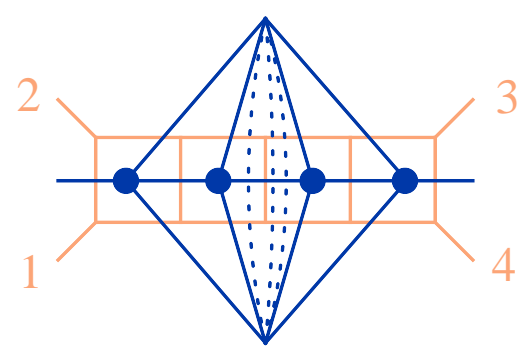

(a)

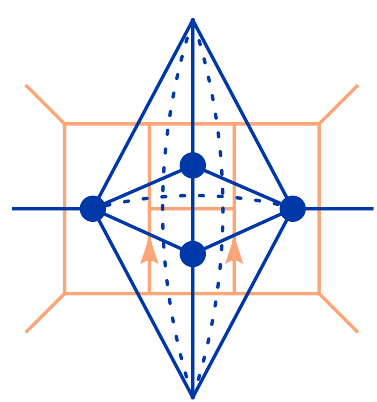

(d)

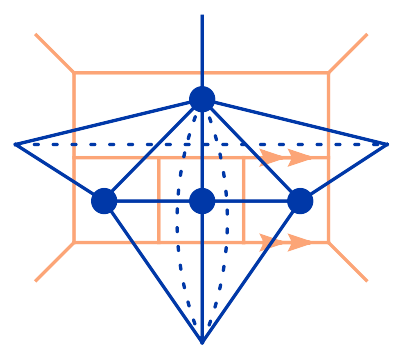

(b)

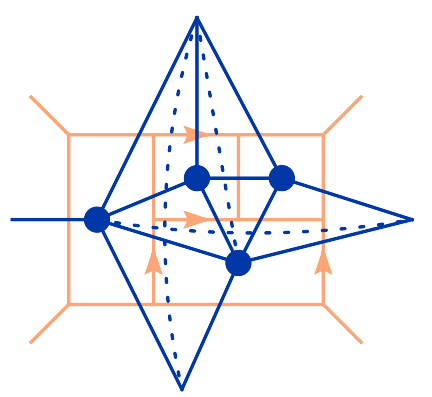

(e)

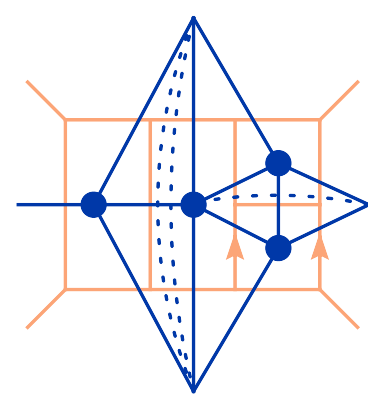

(c)

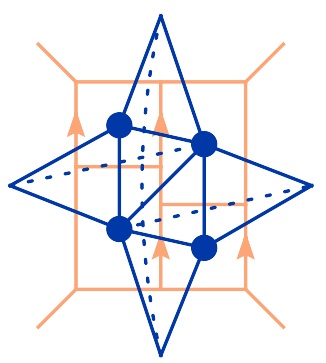

(f)

Figure 17: The rung-rule dual diagrams. A factor of st has been removed.Figure taken from [40].

pseudo-conformal integrals

$$
M_{4}=1+\sum_{\mathcal{I}} a^{l(\mathcal{I})} \sigma_{\mathcal{I}} \mathcal{I},
$$

where the sum runs over all pseudo-conformal integrals (which would be finite off-shell in four dimensions), $\mathcal{I}, a$ is the coupling constant and $l(\mathcal{I})$ is the loop order of a given integral. According to (134), the four-gluon amplitude would be fixed by the relative coefficients $\sigma_{\mathcal{I}}$ coming with the pseudo-conformal integrals. Can these coefficients be predicted as well? One constraint on them comes from the known exponential form of the divergences of the amplitude, c.f. (109),

$$
\ln M_{4}=-\sum_{l=1}^{\infty} a^{l} \frac{\Gamma_{\text {cusp }}}{(l \epsilon)^{2}}+O(1 / \epsilon) .
$$

The $\epsilon$ expansion of individual integrals at a given loop order $l$ typically contains divergences up to $\epsilon^{-2 l}$. This means that the coefficients in (134) have to be such that when one takes the logarithm, the leading divergences cancel, and at most double poles remain. This relates integrals appearing at different loop orders. A practical problem is that in order to use this relation, one has to be able to extract divergences of integrals. It would be highly desirable to have an efficient way of determining at least the leading singularities of a given integral in order to fix some of the relative coefficients (c.f. [168, 169, 167] for related work).

Recall that $\Gamma_{\text {cusp }}$, the coefficient of the double pole, is predicted from a (conjectured) integrable model [30]. This implies that so should be the coefficients $\sigma_{\mathcal{I}}$ in (134), since they implicitly determine $\Gamma_{\text {cusp. }}$. At the moment, integrability is mainly applied to determine the spectrum of anomalous dimensions. Solving the problem of determining the coefficients $\sigma_{\mathcal{I}}$ would be an 


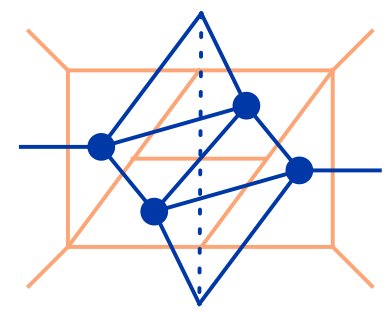

$\left(\mathrm{d}_{2}\right)$

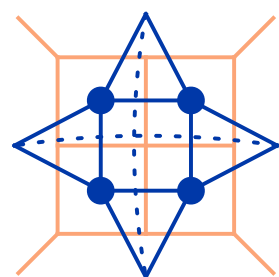

$\left(\mathrm{f}_{2}\right)$

Figure 18: The non-rung rule dual diagrams. A factor of st has been removed. Figure taken from [40].

important step towards extending the use of integrability to the computation of scattering amplitudes.

We studied four-gluon scattering amplitudes. For more than four gluons, one can also define the notion of pseudo-conformal integrals, and the integrals appearing in five- and six-gluon MHV scattering amplitudes up to two loops seem to have this property [76, 92]. This is certainly worth further study.

To summarise, the discovery of this hidden structure in (four-)gluon amplitudes in $\mathcal{N}=4$ SYM is one of the results of this thesis. One can say that the 'conformal rule' (meaning that the amplitude is constructed from a linear combination of 'pseudo-conformal' integrals only) is an improvement over the 'rung-rule' 39, which fails starting from four loops 18. The conformal rule works at least to four loops and possibly at five loops 78 . Will this new rule also fail at some loop order? We like to think that the 'conformal rule' is correct to all loops. Support for this expectation comes from the duality between Wilson loops and gluon amplitudes which will be discussed in section 6. If this duality holds, then the gluon amplitudes inherit the dual conformal properties of the Wilson loops. This might explain why only 'pseudo-conformal' integrals appear in the amplitude. Moreover, it would fix (at least some of) the relative coefficients with which the integrals appear in the amplitude, since then, in addition to (135), the (logarithm of the) sum of the integrals would have to satisfy the same conformal Ward identity as the Wilson loop (see section 8).

\section{Wilson loops and gluon amplitudes at strong coupling}

In this section we summarise work by Alday and Maldacena [79, 80, that motivated the idea of a duality between Wilson loops and gluon amplitudes in $\mathcal{N}=4$ SYM.

\subsection{Prescription for computing scattering amplitudes at strong coupling}

In [79], Alday and Maldacena proposed a way to calculate scattering amplitudes in $\mathcal{N}=4 \mathrm{SYM}$ at strong coupling via the AdS/CFT correspondence [81]. According to [79], gluon scattering at strong coupling is described by the scattering of open strings ending on a $D$-brane in $A d S_{5}$ space with the metric

$$
d s^{2}=R^{2}\left[\frac{d x_{3+1}^{2}+d z^{2}}{z^{2}}\right] .
$$

\footnotetext{
${ }^{18}$ It still predicts correctly the appearance of the rung-rule diagrams, together with theirs prefactors, but it misses other diagrams, as was explained above.
} 


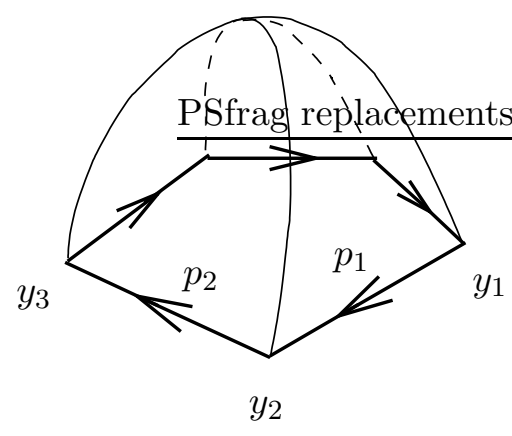

Figure 19: Figure illustrating the minimal surface, whose area $A$ enters Eq. (139).

The $D$-brane is located at $z=z_{\mathrm{IR}}$, where $z_{\mathrm{IR}}$ can be thought of as an infrared regulator. The scattering takes place at fixed angle and at large momentum, and one finds that the solution is dominated by a saddle point of the classical action (see also [170] for a similar computation in flat space).

It turns out that the boundary conditions are easier to describe after changing variables to T-dual coordinates $y^{\mu}$, for which the boundary conditions are simply

$$
\Delta y^{\mu}=2 \pi k^{\mu} .
$$

Notice that this is precisely the relation between momenta of gluons and the dual coordinate space variables 127 from section 3.2 . Importantly, after defining $r=R^{2} / z$, one finds again an $A d S_{5}$ metric in the T-dual space,

$$
d \tilde{s}^{2}=R^{2}\left[\frac{d y_{\mu} d y^{\mu}+d r^{2}}{r^{2}}\right]
$$

So, in summary, the computation of the gluon amplitudes in the original space was related to a computation in a dual coordinate space, which also has an $A d S_{5}$ metric. The new boundary conditions imply that one should compute a minimal 19 surface that ends on a polygon defined by the coordinates $y^{\mu}$ (which are in turn defined by the momenta of the gluons through (137)), as shown in Fig. 19.

$$
\mathcal{A} \propto \exp \left(-\frac{R^{2}}{2 \pi} A_{\text {min }}\right)=\exp \left(-\frac{\sqrt{\lambda}}{2 \pi} A_{\text {min }}\right) .
$$

The proportionality symbol in (139) stands for the dependence of the amplitude $\mathcal{A}$ on the helicity information of the scattering process.

It was further pointed out [79] that the computation of the minimal surface in (139) is formally similar to the computation of a light-like Wilson loop (defined in the dual $A d S_{5}$ space with coordinates $y_{\mu}$ ) at strong coupling. However, from the AdS (and strong coupling) point of view, one might expect the relation between gluon scattering amplitudes and Wilson loops to break down at subleading orders in $1 / \sqrt{\lambda}$.

\subsection{Computation of 4-cusp Wilson loop at strong coupling}

The computation of the minimal surface in (139) is a non-trivial problem and so far has been achieved explicitly only in the case of $n=4$ gluons. Following [79], we first review the onecusp solution obtained in [82, and then present the solution for four gluons. The quantities

\footnotetext{
${ }^{19}$ Strictly speaking, one should say extremal, see a discussion in 171 .
} 


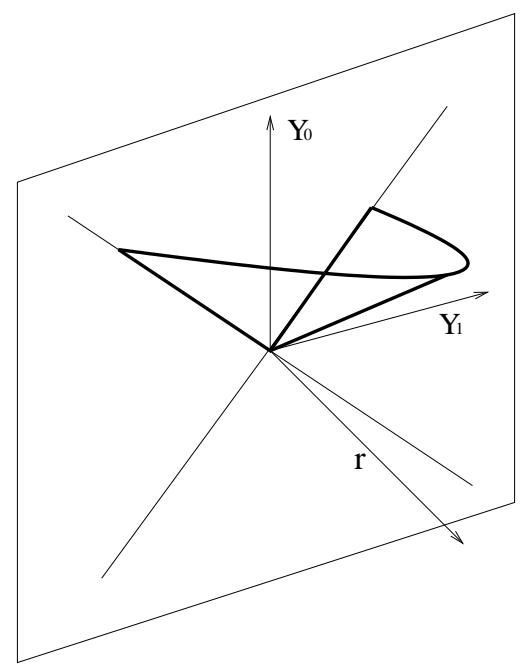

Figure 20: Graphical representation of the one-cusp solution (144). Figure from [79].

entering in (139) are IR divergent, and hence have to be regularised. It turns out that for our purposes it is sufficient to compute the minimal surface for the unregularised string action, and then compute the regularised minimal area by using this unregularised solution. Therefore we present here only the solution of the unregularised minimal surface.

\subsubsection{Single cusp}

Let us consider Wilson lines forming a single cusp on the boundary of $A d S_{5}$. It is sufficient to consider a subspace $A d S_{3}$ of $A d S_{5}$, with the Wilson lines extending along the light-cone,

$$
r=0, \quad y_{1}= \pm y_{0}, \quad y_{0}>0 .
$$

The metric is

$$
d^{2} s=\frac{-d^{2} y_{0}+d^{2} y_{1}+d^{2} r}{r^{2}} .
$$

The Nambu-Goto string action is

$$
S=\frac{R}{2 \pi} \int d s_{1} d s_{2} \sqrt{\operatorname{det}\left(\partial_{a} X^{\mu} \partial_{b} X^{\nu} G_{\mu \nu}\right)} .
$$

In a nonparametric form, where $r$ is expressed through $y_{0}$ and $y_{1}, r=r\left(y_{0}, y_{1}\right)$, it reads

$$
S=\frac{R}{2 \pi} \int d y_{0} d y_{1} \frac{1}{r^{2}} \sqrt{1+\left(\partial_{0} r\right)^{2}+\left(\partial_{1} r\right)^{2}} .
$$

It is straightforward to see that

$$
r\left(y_{0}, y_{1}\right)=\sqrt{2} \sqrt{y_{0}^{2}-y_{1}^{2}}
$$

solves the Euler-Lagrange equations following from (143) with the boundary conditions (140). The minimal surface defined by (144) is shown in Fig. 20.

\subsubsection{Rectangular Wilson loop}

Let us now turn to the rectangular Wilson loop. In the case $s=t$, the minimal surface has to end on a square on the boundary. By scale invariance, the size of the square can be chosen to be 1 , and hence the boundary conditions are

$$
r\left( \pm 1, y_{2}\right)=r\left(y_{1}, \pm 1\right)=0, \quad y_{0}\left( \pm 1, y_{2}\right)= \pm y_{2}, \quad y_{0}\left(y_{1}, \pm 1\right)= \pm y_{1} .
$$


Inspired by the one-cusp solution, Alday and Maldacena found that

$$
y_{0}\left(y_{1}, y_{2}\right)=y_{1} y_{2}, \quad r\left(y_{1}, y_{2}\right)=\sqrt{\left(1-y_{1}^{2}\right)\left(1-y_{2}^{2}\right)}
$$

satisfies the equations of motion following from (142) and solves the boundary condition (145). By applying conformal transformations on the solution (146), one can find the solution for arbitrary $s$ and $t$ [79, 172]. For its explicit expression and more details, we refer the reader to [79].

\subsubsection{Analog of dimensional regularisation at strong coupling}

A subtlety arises when one tries to compute the value of the action (142) by plugging e.g. the solution (146) into it. One finds that the action diverges. Therefore the action has to be regularised. In order to be able to compare to gauge theory results, which are usually derived in dimensional regularisation, Alday and Maldacena introduced an analog of dimensional regularisation at strong coupling. Their regularisation consists in changing the metric (138) to

$$
d s^{2}=\sqrt{\lambda_{D} c_{D}}\left[\frac{d y^{2}+d r^{2}}{r^{2+\epsilon}}\right]
$$

with $\lambda_{D}=\lambda \frac{\mu^{2 \epsilon}}{\left(4 \pi e^{-\gamma} E\right)^{\epsilon}}$ and $c_{D}=2^{4 \epsilon} \pi^{3 \epsilon} \Gamma(2+\epsilon)[79$. This leads to the modified action

$$
S=\frac{\sqrt{\lambda_{D} c_{D}}}{2 \pi} \int \frac{\mathcal{L}_{\epsilon=0}}{r^{\epsilon}}
$$

where $\mathcal{L}_{\epsilon=0}$ is the Lagrangian density for $A d S_{5}$, and $\epsilon<0$ regularised the infrared divergences 20. The Euler-Lagrange equations following from (148) now depend explicitly on $\epsilon$. So, strictly speaking, the solutions of the Euler-Lagrange equations following from (142) obtained in the previous section are no longer valid. In particular, since conformal invariance is broken by the regulator, conformal arguments cannot be used to relate the solution at $s=t$ to that of arbitrary $s$ and $t$.

On the other hand, one can imagine the solutions obtained in this way are still useful if one is only interested in the first few terms of the perturbative expansion in $\epsilon$ of the minimal area. Indeed, Alday and Maldacena argue that one get the accurate value of the minimal surface by plugging the unregularised solution following from (142) into the regularised action, neglecting terms of order $O(\epsilon)$. More precisely, since the divergences come from the cusps, Alday and Maldacena modify the solution near the cusps by an $O(\epsilon)$ term, and in this way they obtain the correct contribution to the finite part of (139). Since this constant is scheme-dependent, we will not be interested in it here. For a more detailed discussion, see [79] 21.

One finds 79 ]

$$
-\frac{\sqrt{\lambda}}{2 \pi} A_{\min }=-\frac{\sqrt{\lambda}}{2 \pi}\left[A_{\mathrm{div}}+\frac{1}{2} \ln ^{2}\left(\frac{s}{t}\right)+C\right],
$$

where $C$ is a scheme-dependent constant. The divergent part is given by $A_{\text {div }}=2 A_{\text {div }, \mathrm{s}}+2 A_{\text {div }, \mathrm{t}}$, where

$$
A_{\mathrm{div}, \mathrm{s}}=\left[-\epsilon^{-2}-\epsilon^{-1} \frac{1-\ln 2}{2}\right]\left(\frac{-s}{\mu^{2}}\right)^{-\epsilon / 2} .
$$

\footnotetext{
${ }^{20}$ Note that in [173] it was found that the dimensional regularisation procedure used in [79] has to be modified at subleading orders in $1 / \sqrt{\lambda}$.

${ }^{21}$ See also 174 for a related discussion of regularisation prescriptions for Wilson loops at strong coupling, when using a cut-off.
} 
Together with the prescription (139), we see that (149) is in agreement with the general form of the divergences of gluon scattering amplitudes, c.f. equation (114). It also reproduces correctly the known strong-coupling value of $\Gamma_{\text {cusp }}$,

$$
\Gamma_{\text {cusp }}(a)=\sqrt{2} \sqrt{a}+O(1,1 / \sqrt{a}), \quad \text { with } \quad a=\frac{g^{2} N}{8 \pi^{2}}=\frac{\lambda}{8 \pi^{2}} .
$$

As was already explained in section 3, the subleading $\epsilon^{-1}$ term and the constant in the finite part of (149) are scheme-dependent, and therefore we are only interested in the functional form of finite part. Most importantly, it is in agreement with the BDS ansatz for the four-gluon amplitude at strong coupling! This constitutes strong evidence that the BDS ansatz holds for the four-gluon amplitude.

The following comments are in order: Extending the result (149) to $n \geq 5$ points turns out to be difficult, and so far no explicit solution for the minimal surface is known. In a later paper, Alday and Maldacena found that the problem simplifies when the number of gluons $n$ is very large. By considering a special contour they were able to relate this problem to a known spacelike contour, and found a disagreement with the BDS ansatz for $n \rightarrow \infty$ [80. This suggests that the BDS ansatz should break down at a certain loop level and for a certain number of gluons. Finally, note that the strong-coupling calculation of [79] is insensitive to the helicity configuration of the scattering amplitude under consideration.

\section{Wilson loops}

Wilson loops were introduced in [175] in the context of quark confinement in strongly coupled QCD. For a Yang-Mills theory with gauge group $S U(N)$, they are defined as

$$
W(C)=\frac{1}{N}\left\langle 0\left|\operatorname{Tr} \mathrm{P} \exp \left(i g \oint_{C} d x^{\mu} A_{\mu}\right)\right| 0\right\rangle .
$$

where $A_{\mu}(x)=A_{\mu}^{a}(x) t^{a}$ is the gauge field, $t^{a}$ are the $S U(N)$ generators in the fundamental representation normalised as $\operatorname{tr}\left(t^{a} t^{b}\right)=\frac{1}{2} \delta^{a b}$, and $\mathrm{P}$ indicates the ordering of the $S U(N)$ indices along the integration contour $C_{n} . W(C)$ is a gauge invariant functional of the contour $C$, along which the gauge field $A_{a}^{\mu}$ is integrated.

\subsection{Renormalisation properties}

\subsubsection{Wilson loops defined on smooth contours}

Let us summarise the renormalisation properties of the Wilson loops (152). In a generic gauge theory with ultraviolet divergences, the gauge field $A_{a}^{\mu}$ is renormalised by multiplicative counterterms, and so is the coupling constant $g$. These counterterms do not depend on the contour $C$. Let us assume that this standard renormalisation has already been carried out and focus on the divergences inherent to the Wilson loop (152).

For smooth contours, the only divergence of (152) is linear (in the cut-off) [36, 176], and proportional to the length of the contour. It can be absorbed in an overall factor,

$$
W(C)=e^{-K L(C)} \times \text { finite },
$$

which can be interpreted as the mass renormalisation of a test particle moving along the contour $C$ [36, 176]. In (153), $L(C)$ is the length of the contour $C$. 
For example, the one-loop correction to (152) is given by 22

$$
W^{(1)}(C)=6^{m} \propto \oint_{C} \oint_{C} \frac{d x_{\mu} d x^{\prime \mu}}{\left(x-x^{\prime}\right)^{2}}
$$

where the double line in the picture denotes the integration contour $C$, and the wiggly line is a free gluon propagator. The integral in (154) has a divergence at $x=x^{\prime}$, which can be regularised by introducing a cut-off $a^{-1}$,

$$
W_{\mathrm{reg}}^{(1)}(C) \propto \oint_{C} \oint_{C} \frac{d x_{\mu} d x^{\prime \mu}}{\left(x-x^{\prime}\right)^{2}+a^{2}}=\int \frac{\dot{x}(s) \cdot \dot{x}(s+t) d s d t}{[x(s+t)-x(s)]^{2}+a^{2}}
$$

Following [36], we choose a parametrisation of the contour with $\dot{x}(s) \cdot \dot{x}(s)=$ const, such that $\dot{x}(s) \cdot \ddot{x}(s)=0$. Since the divergence in (155) comes from the integration near $t=0$, we can write

$$
\begin{aligned}
W_{\text {reg }}^{(1)}(C) & \propto \int d s \dot{x}^{2}(s) \int_{-\Lambda}^{\Lambda} d t \frac{1}{\dot{x}^{2}(s) t^{2}+a^{2}}+\text { finite } \\
& =\frac{\pi}{a} \int d s \sqrt{\dot{x}^{2}(s)}+\text { finite }=\frac{\pi}{a} L(C)+\text { finite } .
\end{aligned}
$$

For smooth contours, there are no divergences apart from the linear divergence [176, 177].

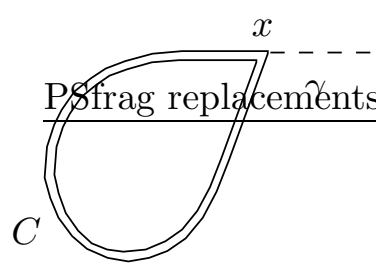

Figure 21: An example of a Wilson loop defined on a contour $C$ having a cusp at the point $x$, the cusp angle being $\gamma$.

\subsubsection{Contours with cusps}

The situation gets more interesting when the contour $C$ is not smooth, but contains one or several cusps, as shown in Fig. 21. In that case, UV (short-distance) divergences associated to each cusp appear [36, 178. For example, a one-loop calculation (for gauge group $S U(N)$ ) gives the result

$$
W(C)=1-2 g^{2} C_{F}[\gamma \cot (\gamma)-1] \ln \left(\frac{L}{a}\right),
$$

where $L$ is the length of the contour, $a$ is a short-distance cut-off, and $\gamma$ is the cusp angle. $C_{F}=$ $t^{a} t^{a}=\left(N^{2}-1\right) /(2 N)$ is the quadratic Casimir of $S U(N)$ in the fundamental representation. The result of [178] is that to any order in the coupling constant, the new divergences can be removed by a multiplicative renormalisation,

$$
W_{R}(C)=Z(\gamma) W(C)
$$

The divergences of $W_{R}(C)$ depend only locally on the contour $C$, through the cusp angle $\gamma$. The locality of the counterterms persists when there are several cusps with cusp angles $\gamma_{i}$. In that case, the multiplicative renormalisation factor

$$
Z\left(\gamma_{1}, \ldots, \gamma_{n}\right)=Z\left(\gamma_{1}\right) \cdots Z\left(\gamma_{n}\right),
$$

\footnotetext{
${ }^{22} \operatorname{In}(154)$ and the following we neglect a trivial normalisation factor .
} 
factorises into a product of renormalisation factors, one for each cusp. Thus, there are no 'anomalous' divergences that depend non-locally on the contour 23.

In [180, 38] a renormalisation group equation for Wilson loops with cusps was derived. It reads

$$
\left(\mu \frac{\partial}{\partial \mu}+\beta\left(g_{\mathrm{R}}\right) \frac{\partial}{\partial g_{\mathrm{R}}}+\Gamma_{\text {cusp }}\left(\gamma, g_{\mathrm{R}}\right)\right) W_{\mathrm{R}}\left(L \mu, \gamma,\{\eta\}, g_{\mathrm{R}}\right)=0 .
$$

Here $g_{\mathrm{R}}$ is the renormalised coupling and

$$
\Gamma\left(\gamma, g_{\mathrm{R}}\right)=\lim _{\epsilon \rightarrow 0} Z \mu \frac{\partial}{\partial \mu} Z^{-1}
$$

From (161) one can derive a renormalisation group equation for $Z^{-1}$,

$$
\left[\beta\left(g_{\mathrm{R}}, \epsilon\right) \frac{\partial}{\partial g_{\mathrm{R}}}-\Gamma\left(\gamma, g_{\mathrm{R}}, \epsilon\right)\right] Z^{-1}\left(\gamma, g_{\mathrm{R}}, \epsilon\right)=0 .
$$

Its solution is

$$
Z^{-1}\left(\gamma, g_{\mathrm{R}}, \epsilon\right)=\exp \left[\int_{0}^{g_{\mathrm{R}}} d g^{\prime} \Gamma\left(\gamma, g^{\prime}, \epsilon\right) / \beta\left(g^{\prime}, \epsilon\right)\right] .
$$

In $\mathcal{N}=4 \mathrm{SYM}$ in dimension $D=4-2 \epsilon$, and when using a scheme where $\beta\left(g_{\mathrm{R}}, \epsilon\right)=-g_{\mathrm{R}} \epsilon$, the integral in (163) can be done explicitly,

$$
Z\left(\gamma, g_{\mathrm{R}}, \epsilon\right)=\exp \left[\sum_{n=1}^{\infty} \frac{g^{2 n}}{2 n} \frac{\Gamma^{(n)}(\gamma, \epsilon)}{\epsilon}\right],
$$

where we expanded $\Gamma\left(\gamma, g_{\mathrm{R}}, \epsilon\right)=\sum_{n=0}^{\infty} g^{2 n} \Gamma^{(n)}(\gamma, \epsilon)$. One can define a $Z$-factor in analogue with the MS-scheme by requiring that $\Gamma\left(\gamma, g_{\mathrm{R}}, \epsilon\right)=\Gamma\left(\gamma, g_{\mathrm{R}}\right)$. So we have seen that the renormalisation group equation (162) implies the exponentiation of the divergent part of the Wilson loop, and in $\mathcal{N}=4 \mathrm{SYM}$ it takes the particular simple form (164).

The Wilson loops studied in this report are defined in Minkowski space, with cusp having an infinite cusp angle. These will be studied in more detail in section 5.4 .

We mention that additional divergences can appear if one considers instead of a closed contour $C$ an open line. In that case one looses gauge independence, and there are logarithmic divergences associated to the endpoints [176].

We also mention the interesting case of crossed contours (i.e., with self-intersections) [178, 181. For these, there are additional divergences associated to the self-intersection. It is found that the loop with the self-intersection can mix with the product of two similar loops [178, which differ from the initial one by a different ordering of the colour indices.

\subsection{Wilson loops in the AdS/CFT correspondence}

The study of Wilson loops in the AdS/CFT correspondence started with the paper [81] (see also [182]). There, Maldacena showed how to compute a particular kind of Wilson loop in $\mathcal{N}=4$ SYM at strong coupling, using the AdS/CFT correspondence. The Maldacena-Wilson loop is similar to (4), but comprises an additional term involving scalars,

$$
W(C)=\frac{1}{N}\left\langle 0\left|\operatorname{Tr} \mathrm{P} \exp \left(i g \oint_{C} d s\left[\dot{x}^{\mu} A_{\mu}+|\dot{x}| \theta^{I} \phi_{I}\right]\right)\right| 0\right\rangle .
$$

\footnotetext{
${ }^{23}$ This can be also understood in the formalism of [179, 177, where the nonlocal Wilson loop is reformulated in terms local fermionic degrees of freedom.
} 
The $\theta^{I}$ in (165) parametrise $S_{5}$ of $A d S_{5} \times S_{5}$, i.e. $\theta^{I} \theta_{I}=1$.

Depending on the shape of the contour, the Maldacena-Wilson loop can be invariant under a certain number of supersymmetries. For example, half of the supersymmetries are preserved if $\dot{x}^{\mu}=$ const, i.e. if the contour $C$ is a straight line 24. Such Wilson loops were studied in 183 . Perturbative calculations up to order $g^{4}$ and a strong coupling calculation using AdS/CFT suggest that

$$
W(C)_{\text {straight line }}=1,
$$

i.e. that the loop corrections vanish identically. This is in close analogy with two- and threepoint functions of $1 / 2$-BPS operators, see section 2.5, which are also invariant under half of the supersymmetry. For Wilson loops that are invariant under less supersymmetry, see e.g. [184].

In this report we will study Wilson loops with light-like contours, i.e. $|\dot{x}|=0$. In this case (165) reduces to the usual definition of the Wilson loop (4). Therefore we will not expand on the discussion of the Maldacena-Wilson loop. For reviews, see [185, 186] and also section 10 of [187.

\subsection{Loop equations}

Another development concerning Wilson loops is the study of loop equations. These describe the behaviour of the loops under small deformations of the contour $C$. We refer the interested reader to the literature [188, 189].

\subsection{Light-like Wilson loops}

\subsubsection{Definitions}

In this report, we consider $\mathcal{N}=4 \mathrm{SYM}$ theory with $S U(N)$ gauge group in Minkowski space. The central object of our consideration is the light-like Wilson loop defined as

$$
W\left(C_{n}\right)=\frac{1}{N}\left\langle 0\left|\operatorname{Tr} \mathrm{P} \exp \left(i \oint_{C_{n}} d x^{\mu} A_{\mu}(x)\right)\right| 0\right\rangle,
$$

where $C_{n}$ is a polygon with $n$ cusps. The $n$ segments of this polygon $C_{n}=\bigcup_{i=1}^{n} \ell_{i}$ joining the cusp points $x_{i}^{\mu}($ with $i=1,2, \ldots, n)$

$$
\ell_{i}=\left\{x^{\mu}\left(\tau_{i}\right)=\tau_{i} x_{i}^{\mu}+\left(1-\tau_{i}\right) x_{i+1}^{\mu} \mid \tau_{i} \in[0,1]\right\},
$$

are all light-like, i.e. $x_{i, i+1}^{2}=0$. Such Wilson loops were considered for the first time in [85] for $n=4$ points in the special kinematics $x_{13}^{2}=-x_{24}^{2}$.

\subsubsection{Cusp singularities}

We already saw in section 5.1 .2 that cusps cause specific ultraviolet divergences (UV) to appear in Wilson loops. The fact that the cusp edges are light-like makes these divergences even more severe [85]. In order to get some insight into the structure of these divergences, we start by giving a one-loop example. Later on in this section we give arguments which lead to the all-order form of the divergences.

\section{One-loop example}

\footnotetext{
${ }^{24}$ One can imagine that this Wilson 'loop' is closed at infinity.
} 


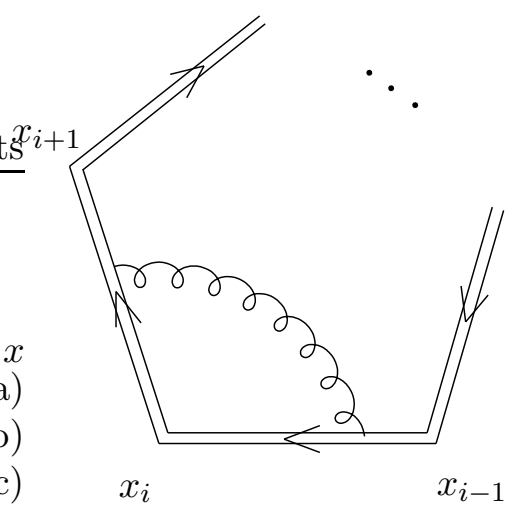

Figure 22: The Feynman diagram contributing to the one-loop divergence at the cusp point $x_{i}$. The double line depicts the integration contour $C_{n}$, the wiggly line the gluon propagator.

Let us first discuss the origin of the cusp singularities in the $n$-gonal Wilson loop $W\left(C_{n}\right)$ to the lowest order in the coupling constant. According to definition (167), it is given by a double contour integral,

$$
W\left(C_{n}\right)=1+\frac{1}{2}(i g)^{2} C_{F} \oint_{C_{n}} d x^{\mu} \oint_{C_{n}} d y^{\nu} D_{\mu \nu}(x-y)+O\left(g^{4}\right) .
$$

Here $C_{F}=\left(N^{2}-1\right) /(2 N)$ is the quadratic Casimir of $S U(N)$ in the fundamental representation and $D_{\mu \nu}(x-y)$ is the gluon propagator in the coordinate representation

$$
\left\langle A_{\mu}^{a}(x) A_{\nu}^{b}(0)\right\rangle=g^{2} \delta^{a b} D_{\mu \nu}(x) .
$$

To regularise the ultraviolet divergences of the integrals entering (169), we use dimensional regularisation with $D=4-2 \epsilon$ and $\epsilon>0$. Also, making use of the gauge invariance of the Wilson loop (167) and for the sake of simplicity, we perform the calculation in the Feynman gauge, where the gluon propagator is given by 25

$$
D_{\mu \nu}(x)=\eta_{\mu \nu} D(x), \quad D(x)=-\frac{\Gamma(1-\epsilon)}{4 \pi^{2}}\left(-x^{2}+i 0\right)^{-1+\epsilon}\left(\mu^{2} \mathrm{e}^{-\gamma_{E}}\right)^{\epsilon} .
$$

The divergences in (169) originate from the integration of the position of the gluon in the vicinity of a light-like cusp in the Feynman diagram shown in Fig. 22 (the other Feynman diagrams at one loop vanish or are finite, see section 6.3). The calculation of this diagram is straightforward [85. Let us compute it for a gluon attached to the edges $p_{i}:=x_{i+1, i}$ and $p_{i-1}:=x_{i, i-1}$, as shown in Fig. 22,

$$
\begin{aligned}
V\left(p_{i-1}, p_{i}\right) & =-\frac{g^{2} C_{F}}{4 \pi^{2}}\left(-e^{-\gamma_{E}} \mu^{2}\right)^{\epsilon} \Gamma(1-\epsilon) \int_{0}^{1} \frac{d x d y\left(p_{i} \cdot p_{i-1}\right)}{\left[\left(p_{i} x+p_{i-1} y\right)^{2}\right]^{1-\epsilon}} \\
& =-\frac{g^{2} C_{F}}{4 \pi^{2}}\left(-e^{-\gamma_{E}} \mu^{2} x_{i+1, i-1}^{2}\right) \frac{\epsilon(1-\epsilon)}{2 \epsilon^{2}} .
\end{aligned}
$$

In (172) we have dropped the $-i 0$ prescription in the gluon propagator because we are only interested in the kinematical region where all $x_{i+1, i-2}^{2}<0$. Adding together the contributions of all cusps we obtain the following one-loop expression for the divergent part of $W\left(C_{n}\right)$ :

$$
W\left(C_{n}\right)=1+\frac{g^{2}}{4 \pi^{2}} C_{F}\left\{-\frac{1}{2 \epsilon^{2}} \sum_{i=1}^{n}\left(-x_{i-1, i+1}^{2} \mu^{2}\right)^{\epsilon}+O\left(\epsilon^{0}\right)\right\}+O\left(g^{4}\right),
$$

\footnotetext{
${ }^{25}$ As in [86], we redefine the conventional dimensional regularization scale as $\mu^{2} \pi \mathrm{e}^{\gamma_{E}} \mapsto \mu^{2}$ to avoid dealing with factors involving $\pi$ and the Euler constant $\gamma_{E}$.
} 
where the periodicity condition

$$
x_{i j}^{2}=x_{i+n, j}^{2}=x_{i, j+n}^{2} \equiv\left(x_{i}-x_{j}\right)^{2}
$$

is tacitly implied. We see from (173) that the leading divergence in $W\left(C_{n}\right)$ is a double pole in $\epsilon$, compared to the single pole in (164). This is due to the light-likeness of the edges defining $C_{n}$, which creates additional divergences. We will discuss the general structure of divergences of light-like Wilson loops in the next section.

\section{All-loop structure}

Generalising (173) to higher loops 26 we find that the cusp singularities appear in $W\left(C_{n}\right)$ at the $l$-th loop-order as poles $\left(a \mu^{2 \epsilon}\right)^{l} / \epsilon^{m}$ with $m \leq 2 l$. Furthermore, $W\left(C_{n}\right)$ can be split into a divergent ('renormalisation') factor $Z_{n}$ and a finite ('renormalised') factor $F_{n}^{(\mathrm{WL})}$ as

$$
\ln W\left(C_{n}\right)=Z_{n}+F_{n}^{(\mathrm{WL})} .
$$

From the studies of renormalisation properties of light-like Wilson loops it is known [85, 190] that cusp singularities exponentiate to all loops and, as a consequence, $Z_{n}$ has the special form 27

$$
Z_{n}=-\frac{1}{4} \sum_{l \geq 1} a^{l} \sum_{i=1}^{n}\left(-x_{i-1, i+1}^{2} \mu^{2}\right)^{l \epsilon}\left(\frac{\Gamma_{\text {cusp }}^{(l)}}{(l \epsilon)^{2}}+\frac{\Gamma^{(l)}}{l \epsilon}\right),
$$

with $a=g^{2} N /\left(8 \pi^{2}\right)$. Here $\Gamma_{\text {cusp }}^{(l)}$ and $\Gamma^{(l)}$ are the expansion coefficients of the cusp anomalous dimension and the so-called collinear anomalous dimension, respectively,

$$
\begin{aligned}
& \Gamma_{\text {cusp }}(a)=\sum_{l \geq 1} a^{l} \Gamma_{\text {cusp }}^{(l)}=2 a-\frac{\pi^{2}}{3} a^{2}+O\left(a^{3}\right), \\
& \Gamma(a)=\sum_{l \geq 1} a^{l} \Gamma^{(l)}=-7 \zeta_{3} a^{2}+O\left(a^{3}\right) .
\end{aligned}
$$

We have already seen at one-loop order that the divergences in $W\left(C_{n}\right)$ are due to the presence of the cusps on the integration contour $C_{n}$. They occur when the gluon propagator in Fig. 22 slides along the contour towards a given cusp point $x_{i}$. The divergences arise when the propagator becomes singular. This happens either when a gluon propagates in the vicinity of the cusp point (short distance divergences), or when a gluon propagates along the light-like segment adjacent to the cusp point (collinear divergences). In these two regimes we encounter, respectively, a double and single pole.

Going to higher orders in the coupling expansion of $W\left(C_{n}\right)$, we immediately realise that the structure of divergences coming from each individual diagram becomes much more complicated. Nevertheless, the divergences originate from the same part of the 'phase space' as at one-loop: from short distances in the vicinity of cusps and from propagation along light-like edges of the contour. The main difficulty in analysing these divergences is due to the fact that in diagrams with several gluons attached to different segments of $C_{n}$ various regimes could be realized simultaneously, thus enhancing the strength of poles in $\epsilon$. This implies, in particular, that individual diagrams could generate higher order poles to $W\left(C_{n}\right)$.

\footnotetext{
${ }^{26}$ For convenience of the presentation, the following formulae are given in the planar limit, with $a=\frac{g^{2} N}{8 \pi^{2}}$. For general $N$, the only difference is that $\Gamma_{\text {cusp }}$ and $\Gamma$ in (177) depend on $N$ as well as on $a$.

${ }^{27}$ Formula (176) follows from the evolution equation (8) of [85].
} 
The simplest way to understand the form of $\ln Z_{n}$ in (176) is to analyse the divergences of the Feynman diagrams not in the Feynman gauge, but in the axial gauge [191] defined as

$$
n \cdot A(x)=0,
$$

with $n^{\mu}$ being an arbitrary vector, $n^{2} \neq 0$. The reason for this is that the contribution from individual Feynman diagrams become less singular in the axial gauge and, most importantly, the potentially divergent graphs have a much simpler topology [192, 193], 28 The axial gauge gluon propagator in the momentum representation is given by the following expression,

$$
\widetilde{D}_{\mu \nu}(k)=-i \frac{d_{\mu \nu}(k)}{k^{2}+i 0}, \quad d_{\mu \nu}^{(A)}(k)=\eta_{\mu \nu}-\frac{k_{\mu} n_{\nu}+k_{\nu} n_{\mu}}{(k n)}+k_{\mu} k_{\nu} \frac{n^{2}}{(k n)^{2}} .
$$

The polarisation tensor satisfies the relation

$$
\eta^{\mu \nu} d_{\mu \nu}^{(A)}(k)=(D-2)+\frac{k^{2} n^{2}}{(k n)^{2}}
$$

(to be compared with the corresponding relation in the Feynman gauge, which is $g^{\mu \nu} d_{\mu \nu}^{(F)}(k)=D$ ) from which we deduce that for $k^{2}=0$, it describes only the physical polarisations of the on-shell gluon. 29

Let us consider a graph in which a gluon is attached to the $i$-th segment. In configuration space, the corresponding effective vertex is described by the contour integral $\int d \tau_{i} p_{i}^{\mu} A_{\mu}\left(x_{i}-\right.$ $\left.p_{i} \tau_{i}\right)$. In the momentum representation, the same vertex reads $\int d \tau_{i} p_{i}^{\mu} \tilde{A}_{\mu}(k) \mathrm{e}^{i k\left(x_{i}-p_{i} \tau_{i}\right)}$ where the field $\tilde{A}_{\mu}(k)$ describes all possible polarisations (2 longitudinal and $D-2$ transverse) of the gluon with momentum $k^{\mu}$. Let us examine the collinear regime, when the gluon propagates along the light-like direction $p_{i}^{\mu}$. The fact that the gluon momentum is collinear, $k^{\mu} \sim p_{i}^{\mu}$, implies that it propagates close to the light-cone and, therefore, has a small virtuality $k^{2}$. Furthermore, since $p_{i}^{\mu} \tilde{A}_{\mu}(k) \sim k^{\mu} \tilde{A}_{\mu}(k)$ we conclude that the contribution of the transverse polarization of the gluon is suppressed as compared to the longitudinal ones. In other words, the most singular contribution in the collinear limit comes from the longitudinal components of the gauge field $\tilde{A}_{\mu}(k)$. The properties of the latter depend on the gauge, however. To see this, let us examine the form of the emission vertex in the Feynman and in the axial gauge. In the underlying Feynman integral, the gauge field $\tilde{A}_{\mu}(k)$ will be replaced by the propagator $\tilde{D}_{\mu \nu}(k)$, with $\nu$ being the polarisation index at the vertex to which the gluon is attached. In this way, we find that

$$
p_{i}^{\mu} \tilde{D}_{\mu \nu}(k) \sim k^{\mu} \tilde{D}_{\mu \nu}(k)=-i \frac{k^{\mu} d_{\mu \nu}(k)}{k^{2}+i 0}=-\frac{i}{k^{2}} \times \begin{cases}k^{\nu}, & \text { Feynman gauge } \\ k^{2}\left[\frac{k^{\nu} n^{2}}{(k n)^{2}}-\frac{n^{\nu}}{(k n)}\right], & \text { axial gauge }\end{cases}
$$

Since $k^{2} \rightarrow 0$ in the collinear limit, we conclude that the vertex is suppressed in the axial gauge, as compared to the Feynman gauge. This is in perfect agreement with our physical intuition - the propagation of longitudinal polarisations of a gluon with momentum $k^{\mu}$ is suppressed for $k^{2} \rightarrow 0$. This property is rather general and it holds not only for gluons attached to the integration contour, but also for the 'genuine' interaction vertices of the $\mathcal{N}=4 \mathrm{SYM}$ Lagrangian [192, 193. It should not be surprising now that the collinear divergences in the light-like Wilson loop come from graphs of very special topology that we shall explain in a moment.

\footnotetext{
${ }^{28}$ Note that the contribution of individual Feynman diagrams is gauge dependent and it is only their total sum that is gauge invariant.

${ }^{29}$ That is the reason why the axial gauge is called physical.
} 


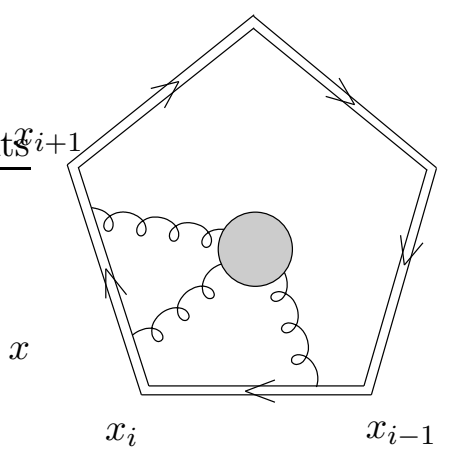

(a)

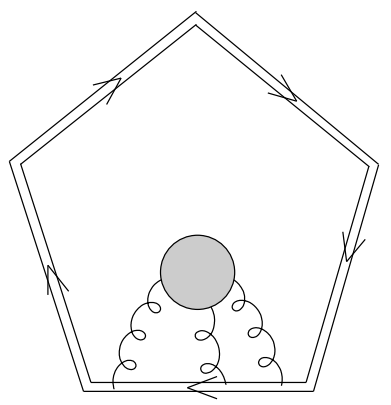

(b)

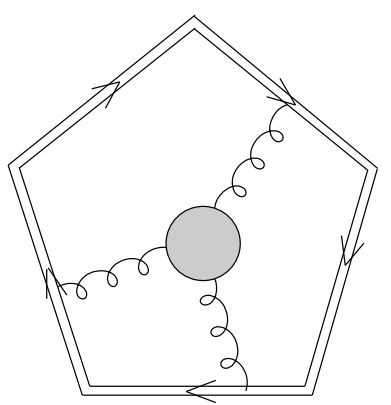

(c)

Figure 23: Maximally non-Abelian Feynman diagrams of different topology ('webs') contributing to $\ln W\left(C_{n}\right)$. In the axial gauge, the vertex-type diagram (a) generates simple pole; the self-energy type diagram (b) generates double pole in $\epsilon$; the diagram (c) with gluons attached to three and more segments is finite.

Another piece of information that will be extensively used in our analysis comes from the non-Abelian exponentiation property of Wilson loops [176, 194, 195]. It follows from the combinatorial properties of the path-ordered exponential and it is not sensitive to the particular form of the Lagrangian of the underlying gauge theory. For an arbitrary integration contour $C$ it can be formulated as follows:

$$
\langle W(C)\rangle=1+\sum_{k=1}^{\infty}\left(\frac{g^{2}}{4 \pi^{2}}\right)^{k} W^{(k)}=\exp \left[\sum_{k=1}^{\infty}\left(\frac{g^{2}}{4 \pi^{2}}\right)^{k} c^{(k)} w^{(k)}\right] .
$$

Here $W^{(k)}$ denote the perturbative corrections to the Wilson loop, while $c^{(k)} w^{(k)}$ are given by the contribution to $W^{(k)}$ from 'webs' $w^{(k)}$ with the 'maximally non-Abelian' color factor $c^{(k)}$. To the first few orders, $k=1,2,3$, the maximally non-Abelian color factor takes the form $c^{(k)}=C_{F} N^{k-1}$, but starting from $k=4$ loops it is not expressible in terms of simple Casimir operators. Naively, one can think of the 'webs' $w^{(k)}$ as of Feynman diagrams with maximally interconnected gluon lines. For the precise definition of 'webs' we refer the interested reader to [194.

Let us now return to the analysis of the cusp divergences of the light-like Wilson loops and take advantage of both the axial gauge and the exponentiation (182). A characteristic feature of the 'webs' following from their maximal non-Abelian nature is that the corresponding Feynman integrals have 'maximally complicated' momentum loop flow, e.g. they cannot be factorised into a product of integrals. When applied to the light-like Wilson loop, this has the following remarkable consequences in the axial gauge:

- the 'webs' do not contain nested divergent subgraphs;

- each web produces a double pole in $\epsilon$ at most;

- the divergent contribution only comes from 'webs' localised at the cusp points as shown in Fig. 23(a) and (b).

These properties imply that the divergent part of $\ln W\left(C_{n}\right)$ is given by a sum over the cusp points $x_{i}^{\mu}$ with each cusp producing a double and single pole contribution. Moreover, the corresponding residue depend on $x_{i-1, i+1}^{2}$ - the only kinematical invariant that one can built out of three vectors $x_{i-1}^{\mu}, x_{i}^{\mu}$ and $x_{i+1}^{\mu}$ satisfying $x_{i-1, i}^{2}=x_{i, i+1}^{2}=0$. In this way, we arrive at the known relation (176) for the divergent part of the light-like Wilson loop. 
Our consideration relied on the analysis of Feynman diagrams at weak coupling. It was recently shown in Refs. [82, 196, 197] that the structure of divergences of $\ln W\left(C_{n}\right)$ remains the same even at strong coupling.

\section{Duality between Wilson loops and gluon amplitudes}

In this section we formulate and discuss the main point of this thesis - the proposed duality relation between planar MHV amplitudes, $\mathcal{M}_{n}^{(\mathrm{MHV})}$, and light-like Wilson loops, $W\left(C_{n}\right)$. As was already mentioned in section 3, it is known that the leading infrared (IR) divergences of gluon amplitudes are in direct equivalence with the leading ultraviolet divergences of Wilson loops. We will illustrate this relationship by a one-loop example in section 6.1. In section 6.2 we state the duality relation between gluon amplitudes and Wilson loops.

\subsection{IR divergences and their relation to Wilson loops}

Infrared divergences of scattering amplitudes can be understood in terms of Wilson loops. For non-Abelian gauge theories, this property was found in [198, 199, 37]. It is known [68, 70] that the IR divergent part of planar scattering amplitudes factorises into a product of form factors, and therefore it is sufficient to understand the relationship between IR divergences of form factors and a particular kind of Wilson lines.

Here, we will give a one-loop example, following to some extent [73] 30. Let us take for simplicity a form factor of massive quarks, with on-shell momenta $p_{1}^{\mu}$ and $-p_{2}^{\mu}, p_{1}^{2}=p_{2}^{2}=m^{2}$. At tree level, this form factor is simply given by

$$
\Gamma_{0}^{\alpha}=f_{a b c} \bar{v}_{2}\left(p_{2}\right) \gamma^{\alpha} u\left(p_{1}\right) \text {. }
$$

At one loop, IR divergences come from the vertex diagram shown in Fig. 24. only. Its contribution

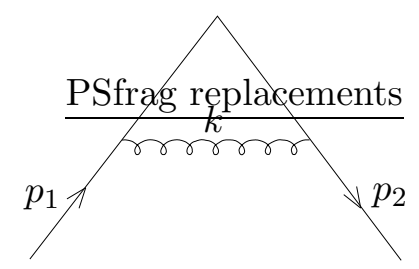

Figure 24: Contribution to the quark form factor at one loop.

is given by

$$
\Gamma_{1}^{\alpha}=f_{a b c} \frac{g^{2}}{2} C_{F} \int \frac{d^{D} k}{(2 \pi)^{D}} \frac{\bar{v}\left(p_{2}\right) \gamma^{\mu}\left(\not p p_{2}-\not k+m\right) \gamma^{\alpha}\left(\not p_{1}-\not k+m\right) \gamma^{\nu} u\left(p_{1}\right)}{\left[\left(p_{1}-k\right)^{2}-m^{2}+i 0\right]\left[\left(p_{2}-k\right)^{2}-m^{2}+i 0\right]} \tilde{D}_{\mu \nu}(k),
$$

where $\tilde{D}^{\mu \nu}(k)$ is the momentum space gluon propagator, e.g. $\tilde{D}^{\mu \nu}(k)=\eta^{\mu \nu} / k^{2}$ in the Feynman gauge. The divergence in (184) originates from the soft region of loop momenta, $k \ll m$. In dimensional regularisation, it manifests itself as a pole in $\epsilon, \int_{\Sigma} d^{D} k / k^{4} \sim 1 /(4-D)$, where $\Sigma$ denotes the integration region for small $k 31$ In that region, we can neglect $k$ in the numerator, and make the approximation

$$
\left(p_{1}-k\right)^{2}-m^{2} \approx-2\left(p_{1} \cdot k\right),
$$

\footnotetext{
${ }^{30}$ I am indebted to Gregory Korchemsky for enlightening explanations, on which this section is based.

${ }^{31}$ Note that for massless quarks, $m^{2}=0$, there are additional collinear divergences, which is why the leading pole is double in that case.
} 
and similarly for the other quark propagator. Using in addition the Dirac equations of motion for the on-shell quarks, e.g. $\left(\not p_{1}-m\right) u\left(p_{1}\right)=0$, we obtain

$$
\Gamma_{1}^{\alpha \mathrm{IR}}=f_{a b c} \frac{g^{2}}{2} C_{F} \bar{v}_{2}\left(p_{2}\right) \gamma^{\alpha} u\left(p_{1}\right) \int_{\Sigma} \frac{d^{D} k}{(2 \pi)^{D}} \frac{p_{1}^{\nu}}{\left(-p_{1} \cdot k+i 0\right)} \frac{p_{2}^{\mu}}{\left(-p_{2} \cdot k+i 0\right)} \tilde{D}_{\mu \nu}(k) .
$$

We can interpret the integral in (186) as coming from the vertex diagram 24, with the quarkantiquark-gluon vertex replaced by the eikonal vertex

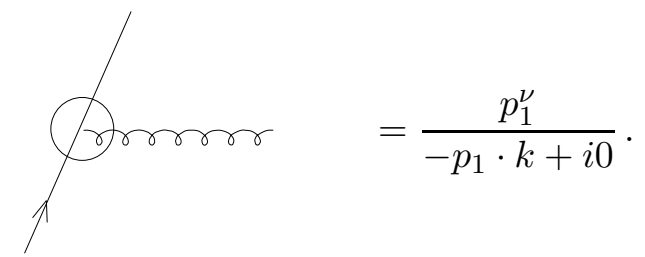

Notice that the quark helicity is not modified by this eikonal vertex, and $\Gamma_{1}^{\alpha \mathrm{IR}}$ is proportional to the tree level form factor in (183). From now on, we will drop the tree-level factor, i.e. we consider

$$
I_{\Sigma}=\frac{g^{2}}{2} C_{F} \int_{\Sigma} \frac{d^{D} k}{(2 \pi)^{D}} \frac{p_{1}^{\nu}}{\left(-p_{1} \cdot k+i 0\right)} \frac{p_{2}^{\mu}}{\left(-p_{2} \cdot k+i 0\right)} \tilde{D}_{\mu \nu}(k)
$$

Note that from the invariance of (188) under rescalings $p_{1} \rightarrow \lambda_{1} p_{1}$ and $p_{2} \rightarrow \lambda_{2} p_{2}$, one can deduce that it depends on $p_{1}^{\mu}$ and $p_{2}^{\mu}$ through the dimensionless variable $\cosh \gamma=\left(p_{1} \cdot p_{2}\right) / \sqrt{p_{1}^{2} p_{2}^{2}}$ only. Geometrically, $\gamma$ corresponds to the cusp angle formed by the momenta $p_{1}$ and $p_{2}$ in Minkowski space.

Now we would like to extend the integration region from $\Sigma$ to the full phase space. In doing so, the integral in (188) acquires additional, artificial ultraviolet (UV) divergences. In dimensional regularisation, the UV and IR divergences cancel, and the integral over the full phase space is zero,

$$
\frac{g^{2}}{2} C_{F} \int \frac{d^{D} k}{(2 \pi)^{D}} \frac{p_{1}^{\nu}}{\left(-p_{1} \cdot k+i 0\right)} \frac{p_{2}^{\mu}}{\left(-p_{2} \cdot k+i 0\right)} \tilde{D}_{\mu \nu}(k)=\Lambda^{\mathrm{IR}}+\Lambda^{\mathrm{UV}}=0 .
$$

So instead of studying the IR divergences of (189) at $k \ll m$, we can equivalently study its UV divergences at $k \gg m$. These UV divergences are well-understood, because they correspond to cusp divergences of Wilson loops, as we will see presently. By using the identity

$$
\frac{1}{-p_{1} \cdot k+i 0}=-i \int_{0}^{\infty} d s_{1} e^{i s_{1}\left(-p_{1} \cdot k+i 0\right)}
$$

and similarly for $-p_{2} \cdot k+i 0$, we can rewrite (189) as

$$
\begin{aligned}
I & =\frac{1}{2}(i g)^{2} C_{F} \int_{0}^{\infty} d s_{1} \int_{0}^{\infty} d s_{2} \int \frac{d^{D} k}{(2 \pi)^{D}} p_{1}^{\mu} p_{2}^{\nu} \tilde{D}_{\mu \nu}(k) e^{i k \cdot\left(-s_{1} p_{1}-s_{2} p_{2}+i 0\right)} \\
& =\frac{1}{2}(i g)^{2} C_{F} \int_{0}^{\infty} d s_{1} \int_{0}^{\infty} d s_{2} p_{1}^{\mu} p_{2}^{\nu} D_{\mu \nu}\left(p_{1} s_{1}+p_{2} s_{2}\right),
\end{aligned}
$$

where in the second line we introduced the Fourier transform of the gluon propagator,

$$
D_{\mu \nu}(x)=\int \frac{d^{D} k}{(2 \pi)^{D}} \tilde{D}_{\mu \nu}(k) e^{i k \cdot(x+i 0)} .
$$

Equation (191) is just the one-loop contribution to a Wilson loop 32

$$
W(C)=\left\langle 0\left|\operatorname{Tr} \mathrm{P} \exp \left(i g \int_{C} d x_{\mu} A^{\mu}(x)\right)\right| 0\right\rangle,
$$

\footnotetext{
${ }^{32}$ Strictly speaking, it is a Wilson line, but one can imagine it is closed at infinity.
} 
defined by the contour $C=C_{1} \cup C_{2}$, where $C_{1}=\left\{s p_{1}, s \in[-\infty, 0]\right\}$ and $C_{2}=\left\{t p_{2}, t \in\right.$ $[0, \infty]\}$. This means that the quarks were effectively replaced by Wilson lines along their classical trajectory.

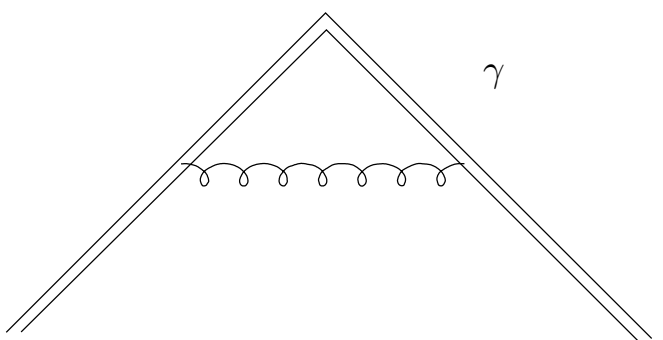

(a)

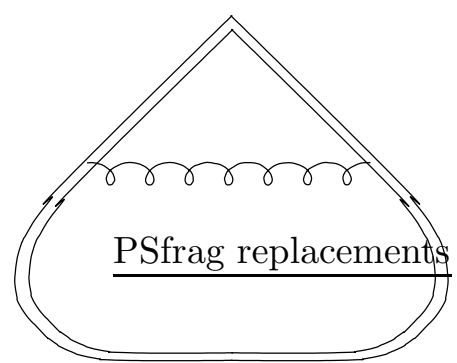

(b)

Figure 25: One-loop contribution to cusped Wilson loop. The double lines denote the integration contour. The UV divergences of the Wilson loop correspond to the IR divergences of the form factor.

After the Fourier transform, the UV divergences of (193) come from the region where $p_{1} s_{1}+$ $p_{2} s_{2} \ll m$, i.e. from the integration region of small $s_{1}, s_{2}$. This corresponds to the situation where the gluon propagator is integrated near the cusp of the Wilson loop. Taking into account the relation between UV and IR divergences (189), we see that the IR divergences of the form factor (184) are equivalently described by the UV divergences of a cusped Wilson loop, as shown in Fig. 25 (a). The latter satisfies a renormalisation group equation [180] (see also section 5.1.2). As explained in section 5.1.2, for $\mathcal{N}=4 \mathrm{SYM}$ this RG equation implies that the divergent part of the Wilson loop (and hence that of the form factor as well) takes the form

$$
Z\left(\gamma, g_{\mathrm{R}}, \epsilon\right)=\exp \left[\sum_{n=1}^{\infty} \frac{g^{2 n}}{2 n} \frac{\Gamma^{(n)}(\gamma, \epsilon)}{\epsilon}\right] .
$$

For an explicit two-loop calculation, see [200].

We remark that since we need only the UV divergences of the Wilson loop shown in Fig. 25 (a), which arise from the integration of gluons near the cusp, we can choose a modified integration contour, as shown in Fig. 25 (b). In this way, the new Wilson loop has only the desired UV divergences, but no IR divergences.

Another important remark is that the definition of the Wilson loop (193) contains an unusual mixture of momenta and coordinates. Indeed, the 'coordinates' of the Wilson loop (193) are defined by the gluon momenta (and formally have 'wrong' canonical dimension). The same is true for the Wilson loops that enter the duality with gluon amplitudes, as we will see in the next section. These Wilson loops 'live' in a dual coordinate space defined by the gluon momenta.

\subsection{Duality relation}

The conjectured duality states that in the planar $\mathcal{N}=4$ SYM theory the finite part of the logarithms of the gluon amplitude and of the Wilson loop are equal (up to an inessential additive constant),

$$
F_{n}^{(\mathrm{MHV})}=F_{n}^{(\mathrm{WL})}+\text { const },
$$

upon the formal identification of the external on-shell gluon momenta in the amplitude with the light-like segments forming the closed polygon $C_{n}$ (the contour of the Wilson loop),

$$
p_{i}^{\mu}:=x_{i+1}^{\mu}-x_{i}^{\mu} .
$$


Thus, the Mandelstam variables for the scattering amplitudes $t_{i}^{[j]}=\left(p_{i}+\ldots p_{i+j-1}\right)^{2}$ are related to the distances $x_{i j}^{2}$ between two cusp points on the integration contour of $W\left(C_{n}\right)$ are follows,

$$
t_{i}^{[j]} / t_{k}^{[l]}:=x_{i, i+j}^{2} / x_{k, k+l}^{2} .
$$

The divergent parts of the scattering amplitudes and the light-like Wilson loops are also related to each other but the relationship is more subtle since the two objects are defined in two different schemes (infrared regularisation for the amplitudes and ultraviolet regularisation for the Wilson loops), both based on dimensional regularisation. From the previous section (see also the more detailed discussion in [91]) we know that the leading IR divergence of the amplitude (the coefficient of the double pole $\epsilon_{\mathrm{IR}}^{-2}$ in equation (114)) coincides with the leading UV divergence of the Wilson loop (the coefficient of the double pole $\epsilon_{\mathrm{UV}}^{-2}$ in equation (176)), since both are controlled by the universal cusp anomalous dimension $\Gamma_{\text {cusp }}(a)$. One can also achieve a matching of the coefficients of the subleading simple poles corresponding to the (non-universal) collinear anomalous dimensions $G(a)$ and $\Gamma(a)$. 33 To this end one relates the parameters of the two different renormalization schemes as follows,

$$
x_{i, i+2}^{2} \mu_{\mathrm{UV}}^{2}:=t_{i}^{[2]} / \mu_{\mathrm{IR}}^{2} e^{\gamma(a)}, \quad \epsilon_{\mathrm{UV}}:=-\epsilon_{\mathrm{IR}} e^{\epsilon_{\mathrm{IR}} \delta(a)} .
$$

Here the functions $\gamma(a)$ and $\delta(a)$ are chosen in a way to compensate the mismatch between $G(a)$ and $\Gamma(a)$, without creating extra $\mu$-dependent finite terms [91. It should be stressed that this procedure is not analogous to comparing two different renormalisation schemes for the computation of the same divergent object. It is rather a change of variables (regularisation parameters) which allows us to compare two different objects computed in two different schemes. Another such change of variables is the identification (196) of the particle momenta with the light-like segments on the Wilson loop contour.

The duality relation (195) was inspired by the prescription of Alday and Maldacena for computing gluon scattering amplitudes at strong coupling [79]. This prescription essentially recasts the amplitudes into light-like Wilson loops in the dual variables (196), as discussed in section 4. A priori, one would expect the strong coupling relation between gluon amplitudes and Wilson loops to receive $1 / \sqrt{\lambda}$ corrections, which might spoil the relation at weak coupling [79]. Nevertheless, in 83 it was found that at one loop and for four points the Wilson loop and the gluon amplitude agree, which lead to the idea that the duality might also be true perturbatively. In 84 the duality at one loop was shown to apply to $n$-point amplitudes as well. These one-loop results will be reviewed in section 6.3 .

\subsection{Duality at one loop}

We already discussed results of perturbative calculations for gluon amplitudes, in section 3.1.4, Here, we review the Wilson loop calculation at one loop. Let us start by computing for $n=4$ points, following [83]. The perturbative expansion of the Wilson loop

$$
W\left(C_{4}\right)=\frac{1}{N}\left\langle 0\left|\operatorname{Tr} \mathrm{P} \exp \left(i \oint_{C_{4}} d x^{\mu} A_{\mu}(x)\right)\right| 0\right\rangle
$$

to the lowest order in the coupling is given by

$$
W\left(C_{4}\right)=1+\frac{1}{2}(i g)^{2} C_{F} \oint_{C_{4}} d x^{\mu} \oint_{C_{4}} d y^{\nu} D^{\mu \nu}(x-y)+O\left(g^{4}\right),
$$

\footnotetext{
${ }^{33}$ We thank Paul Heslop for turning our attention to the incomplete discussion of this point in the first version of 91. We are also grateful to Lance Dixon for a discussion of the different physical interpretations of the IR and UV simple poles 201].
} 
where $D^{\mu \nu}$ is the free gluon propagator and $C_{F}=\left(N_{c}^{2}-1\right) /\left(2 N_{c}\right)$. Its expression in the Feynman gauge was given in (171). Recall that the contour $C_{4}$ consists of four light-like segments $x_{i, i+1}^{\mu}, x_{i, i+1}^{2}=0$ (with the cyclicity condition $i+4 \equiv i$ for the indices). Hence, the Wilson loop can only depend on the two available Lorentz invariant quantities, $x_{13}^{2}$ and $x_{24}^{2}$. Through (196) they correspond to the Mandelstam variables $s$ and $t$ of the four-gluon amplitude, i.e. $x_{13}^{2}=\left(p_{1}+p_{2}\right)^{2}=s$ and $x_{24}^{2}=\left(p_{2}+p_{3}\right)^{2}=t$.

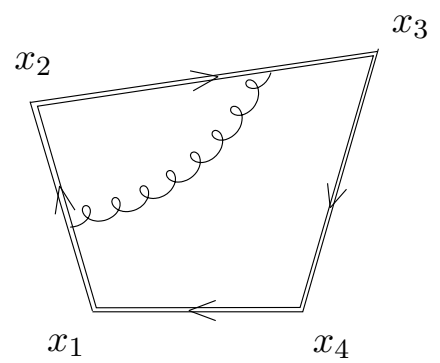

(a)

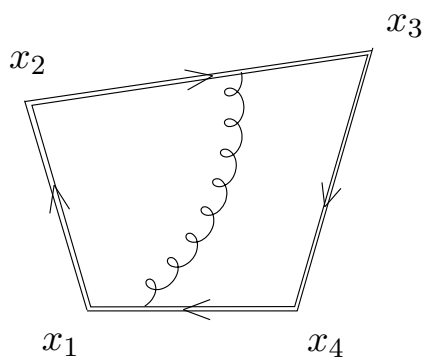

(b)

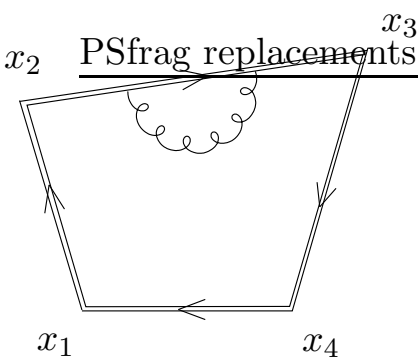

(c)

Figure 26: The Feynman diagrams representation of the integrals contributing to (200). The double line depicts the integration contour $C$ and the wiggly line the gluon propagator. Figure adapted from [83].

The Feynman diagrams contribution to (200) are shown in Fig. 26. In the Feynman gauge, diagram (c) vanishes due to the light-likeness of the edge the gluon is attached to. The vertex type diagram (a) was already calculated in section 5.4.2, In application to the four-point case we find from (173)

$V\left(p_{1}, p_{2}\right)+V\left(p_{2}, p_{3}\right)+V\left(p_{3}, p_{4}\right)+V\left(p_{4}, p_{1}\right)=-\frac{g^{2} C_{F}}{4 \pi^{2}} \frac{\Gamma(1-\epsilon)}{\epsilon^{2}}\left[\left(-e^{-\gamma_{E}} \mu^{2} s\right)^{\epsilon}+\left(-e^{-\gamma_{E}} \mu^{2} t\right)^{\epsilon}\right]$

Let us now turn to diagram (b), in which the gluon is attached to segments $p_{2}$ and $p_{4}$. This diagram is finite for $\varepsilon \rightarrow 0$, so we evaluate it in $D=4$ dimensions

$$
I\left(p_{2}, p_{4}\right)=-\frac{g^{2} C_{F}}{4 \pi^{2}} \int_{0}^{1} \frac{d x d y\left(p_{2} \cdot p_{4}\right)}{\left(p_{2} x+p_{4} y+p_{3}\right)^{2}}=\frac{g^{2} C_{F}}{8 \pi^{2}} \int_{0}^{1} \frac{d x d y(s+t)}{t x+s y-(s+t) x y} .
$$

Integration yields

$$
I\left(p_{2}, p_{4}\right)=-\frac{g^{2} C_{F}}{8 \pi^{2}} \int_{0}^{1} \frac{d x}{x-\frac{s}{s+t}}\left[\ln \frac{s}{t}+\ln \frac{1-x}{x}\right]=\frac{g^{2} C_{F}}{16 \pi^{2}}\left[\ln ^{2}(s / t)+\pi^{2}\right],
$$

where we assumed that $s, t<0$. The other diagram of the same topology, with the gluon going from segment $p_{1}$ to $p_{3}$ gives the same contribution, since it is obtained from interchanging $s$ and $t$. Finally, summing up all contributions and expanding in powers of $\epsilon$, we find

$$
W\left(C_{4}\right)=1+\frac{g^{2} C_{F}}{4 \pi^{2}}\left\{-\frac{1}{\epsilon^{2}}\left[\left(-\mu^{2} x_{13}^{2}\right)^{\epsilon}+\left(-\mu^{2} x_{24}^{2}\right)^{\epsilon}\right]+\frac{1}{2} \ln ^{2}\left(\frac{x_{13}^{2}}{x_{24}^{2}}\right)+\frac{\pi^{2}}{3}\right\}
$$

In order to compare with the corresponding gluon amplitude, we write the finite part of $\ln W\left(C_{4}\right)$ at one loop in the planar limit, which amounts to replacing $C_{F} \rightarrow N / 2$, i.e. $g^{2} C_{F} /\left(4 \pi^{2}\right) \rightarrow a$,

$$
\ln W\left(C_{4}\right)=F_{4}^{(\mathrm{WL})}=a\left[\frac{1}{2} \ln ^{2}\left(\frac{x_{13}^{2}}{x_{24}^{2}}\right)+\frac{\pi^{2}}{3}\right]+O\left(a^{2}\right) .
$$

Comparing to the corresponding expression for the four-gluon amplitude (125) and the one-loop value of the cusp anomalous dimension (177), we see that (205) is indeed in agreement with the duality relation (195). Let us stress again that the additive constant in the finite part of (204) is scheme dependent.

To get the $n$-point result, one has to evaluate the finite diagram (b) in more general kinematics [84]. The result is again in agreement with the duality relation (195). 


\subsection{Checks of the duality at two loops and beyond}

We remark that the one-loop Wilson loop calculations presented in the previous section did not use special properties of $\mathcal{N}=4 \mathrm{SYM}$. Indeed, only the free gluon propagator $D^{\mu \nu}(x)$ entered the calculation, and hence the result in other gauge theories would have been the same.

This motivates to extend the checks of the duality (195) beyond one loop, where one can see a difference between e.g. QCD and $\mathcal{N}=4 \mathrm{SYM}$. The remaining sections of this thesis are devoted to this. We report on results that confirm the duality (195) in several non-trivial cases at two loops and beyond.

In [86, Drummond, Korchemsky, Sokatchev and the present author carried out the two-loop computation of the Wilson loop for $n=4$ points, which we will present in section 7 . Technical details of this calculation can be found in appendix B, where the evaluation of each Feynman diagram is shown explicitly. In section 8 we present the results of the publication [87, where we derive all-order Ward identities for the Wilson loops (4). In section 9, we perform a calculation at six points and two loops and compare to the BDS ansatz and a recently available two loop calculation of the six-gluon MHV amplitude.

\section{Two loop tests of the duality}

\subsection{Rectangular Wilson loop}

In this section we present the result of the calculation of the rectangular Wilson loop

$$
W\left(C_{4}\right)=\frac{1}{N}\left\langle 0\left|\operatorname{Tr} \mathrm{P} \exp \left(i \oint_{C_{4}} d x^{\mu} A_{\mu}(x)\right)\right| 0\right\rangle,
$$

to two loops. The details of the calculation can be found in the appendix.

In order to compute the two-loop Feynman diagrams shown in Fig. 27 we employ the technique described in detail in Refs. [158, 159, 85, 190]. Furthermore, the rectangular light-like Wilson loop under consideration has been already calculated to two loops in [85, 190] in the so-called forward limit $x_{12}^{\mu}=-x_{34}^{\mu}$, or equivalently $x_{13}^{2}=-x_{24}^{2}$, in which the contour $C_{4}$ takes the form of a rhombus.

The vertex-like diagrams shown in Fig. 27(c), 27(d) and 27(f) only depend on the distance $x_{13}^{2}$ and hence we can take the results from [85, 190]. The Feynman diagrams shown in Fig. 27 (h) - 27(l) are proportional to scalar products $\left(x_{12} \cdot x_{34}\right)$ and/or $\left(x_{23} \cdot x_{14}\right)$ and, therefore, they vanish in the limit $x_{12}^{\mu}=-x_{34}^{\mu}$ due to $x_{i, i+1}^{2}=0$. For generic forms of $C_{4}$, these diagrams have to be calculated anew. Similarly, we also have to reexamine the contribution of diagrams shown in Figs. 27(e) and 27(g). Finally, the calculation in Ref. [85, 190] was performed within the conventional dimensional regularisation (DREG) scheme. To preserve supersymmetry, we have to use instead the dimensional reduction (DRED) scheme. The change of scheme only affects the diagrams shown in Figs. 27(c) and 27(k) which involve an internal gluon loop [16].

The results of our calculation can be summarised as follows. Thanks to the non-Abelian exponentiation (182), the two-loop expression for the (unrenormalised) light-like Wilson loop can be represented as

$$
\ln W\left(C_{4}\right)=\frac{g^{2}}{4 \pi^{2}} C_{F} w^{(1)}+\left(\frac{g^{2}}{4 \pi^{2}}\right)^{2} C_{F} N w^{(2)}+O\left(g^{6}\right) .
$$




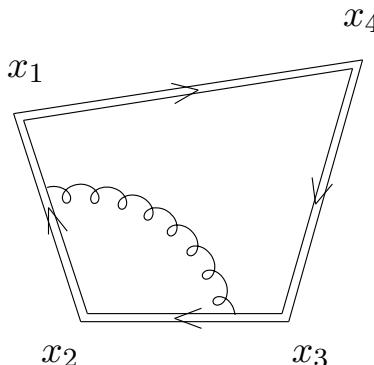

(a)

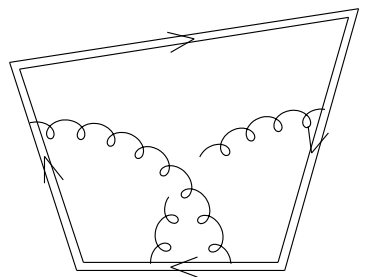

(e)

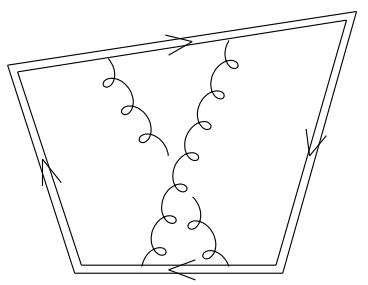

(i)

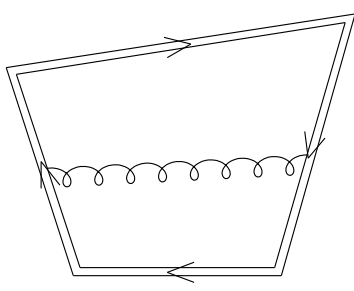

(b)

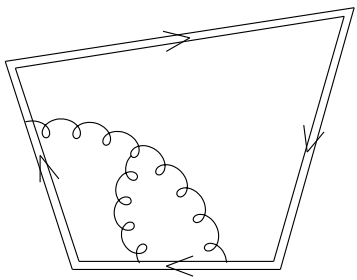

(f)

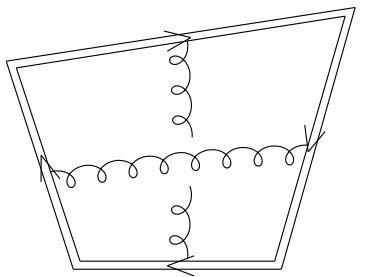

(j)

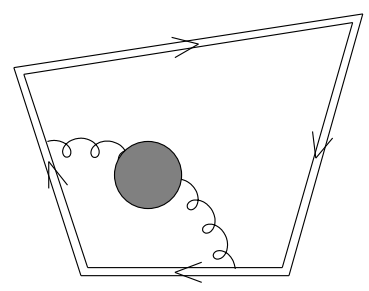

(c)

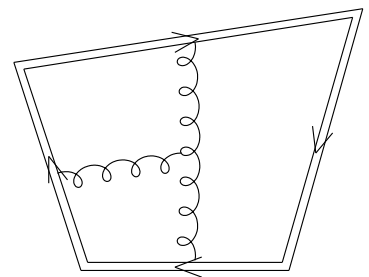

(g)

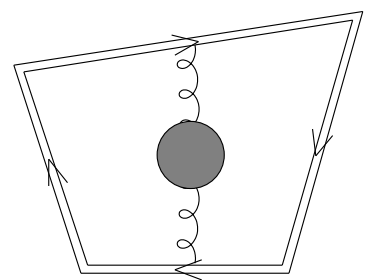

(k)

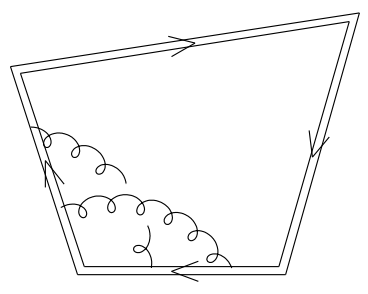

(d)

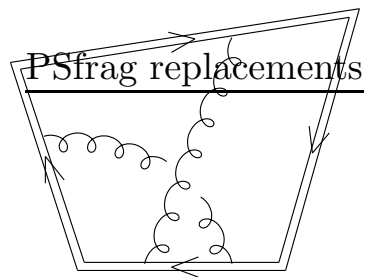

(h)

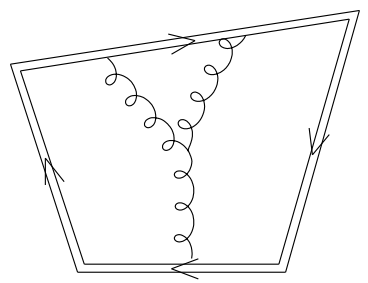

(l)

Figure 27: The Feynman diagrams contributing to $\ln W\left(C_{4}\right)$ to two loops. The double line depicts the integration contour $C_{4}$, the wiggly line the gluon propagator and the blob the one-loop polarization operator.

According to (204) the one-loop correction $w^{(1)}$ is given by

$$
w^{(1)}=-\frac{1}{\epsilon^{2}}\left[\left(-x_{13}^{2} \mu^{2}\right)^{\epsilon}+\left(-x_{24}^{2} \mu^{2}\right)^{\epsilon}\right]+\frac{1}{2} \ln ^{2}\left(\frac{x_{13}^{2}}{x_{24}^{2}}\right)+\frac{\pi^{2}}{3}+O(\epsilon) .
$$

The two-loop correction $w^{(2)}$ is given by a sum over the individual diagrams shown in Fig. 27 plus crossing symmetric diagrams. It is convenient to expand their contributions in powers of $1 / \epsilon$ and separate the UV divergent and finite parts as follows

$$
w^{(2)}=\sum_{\alpha}\left[\left(-x_{13}^{2} \mu^{2}\right)^{2 \epsilon}+\left(-x_{24}^{2} \mu^{2}\right)^{2 \epsilon}\right]\left\{\frac{1}{\epsilon^{4}} A_{-4}^{(\alpha)}+\frac{1}{\epsilon^{3}} A_{-3}^{(\alpha)}+\frac{1}{\epsilon^{2}} A_{-2}^{(\alpha)}+\frac{1}{\epsilon} A_{-1}^{(\alpha)}\right\}+A_{0}^{(\alpha)}+O(\epsilon),
$$

where the sum goes over the two-loop Feynman diagrams shown in Fig. 27(c)-(1). Here $A_{-n}^{(\alpha)}$ (with $0 \leq n \leq 4$ ) are dimensionless functions of the ratio of distances $x_{13}^{2} / x_{24}^{2}$. Making use of (209), we can parametrise the contribution of each individual diagram to the Wilson loop by the set of coefficient functions $A_{-n}^{(\alpha)}$.

- UV divergent $O\left(1 / \epsilon^{4}\right)$ terms only come from the two Feynman diagrams shown in Figs. 27(d) 
and $27(\mathrm{f})$

$$
A_{-4}^{(\mathrm{d})}=-\frac{1}{16}, \quad A_{-4}^{(\mathrm{f})}=\frac{1}{16}
$$

- UV divergent $O\left(1 / \epsilon^{3}\right)$ terms only come from the two Feynman diagrams shown in Figs. 27(c) and $27(f)$

$$
A_{-3}^{(\mathrm{c})}=\frac{1}{8}, \quad A_{-3}^{(\mathrm{f})}=-\frac{1}{8}
$$

- UV divergent $O\left(1 / \epsilon^{2}\right)$ terms only come from the Feynman diagrams shown in Figs. 27(c)27(g)

$$
A_{-2}^{(\mathrm{c})}=\frac{1}{4}, \quad A_{-2}^{(\mathrm{d})}=-\frac{\pi^{2}}{96}, \quad A_{-2}^{(\mathrm{e})}=-\frac{\pi^{2}}{24}, \quad A_{-2}^{(\mathrm{f})}=-\frac{1}{4}+\frac{5}{96} \pi^{2}, \quad A_{-2}^{(\mathrm{g})}=\frac{\pi^{2}}{48}
$$

- UV divergent $O\left(1 / \epsilon^{1}\right)$ terms come from the Feynman diagrams shown in Figs. 27(c)27(h),27(k) and 27(l)

$$
\begin{aligned}
A_{-1}^{(\mathrm{c})} & =\frac{1}{2}+\frac{\pi^{2}}{48}, & A_{-1}^{(\mathrm{d})} & =-\frac{1}{24} \zeta_{3}, \\
A_{-1}^{(\mathrm{e})} & =\frac{1}{2} \zeta_{3}, & A_{-1}^{(\mathrm{f})} & =-\frac{1}{2}-\frac{\pi^{2}}{48}+\frac{7}{24} \zeta_{3}, \\
A_{-1}^{(\mathrm{g})} & =-\frac{1}{8} M_{2}+\frac{1}{8} \zeta_{3}, & A_{-1}^{(\mathrm{h})} & =\frac{1}{4} M_{2}, \\
A_{-1}^{(\mathrm{k})} & =\frac{1}{4} M_{1}, & A_{-1}^{(\mathrm{l})} & =-\frac{1}{4} M_{1}-\frac{1}{8} M_{2}
\end{aligned}
$$

- Finite $O\left(\epsilon^{0}\right)$ terms come from all Feynman diagrams shown in Figs. 27(c)-27(l)

$$
\begin{aligned}
A_{0}^{(\mathrm{c})} & =2+\frac{\pi^{2}}{12}+\frac{1}{6} \zeta_{3}, & A_{0}^{(\mathrm{d})} & =-\frac{7}{2880} \pi^{4}, \\
A_{0}^{(\mathrm{e})} & =-\frac{\pi^{2}}{12} M_{1}-\frac{49}{720} \pi^{4}, & A_{0}^{(\mathrm{f})} & =-2-\frac{\pi^{2}}{12}+\frac{119}{2880} \pi^{4}-\frac{1}{6} \zeta_{3}, \\
A_{0}^{(\mathrm{g})} & =\frac{1}{24} M_{1}^{2}-\frac{1}{4} M_{3}+\frac{7}{360} \pi^{4}, & A_{0}^{(\mathrm{h})} & =\frac{1}{8} M_{1}^{2}+\frac{3}{8} M_{3}+\frac{\pi^{2}}{8} M_{1}, \\
A_{0}^{(\mathrm{i})} & =-\frac{1}{24} M_{1}^{2}, & A_{0}^{(\mathrm{j})} & =-\frac{1}{8} M_{1}^{2} \\
A_{0}^{(\mathrm{k})} & =M_{1}+\frac{1}{2} M_{2}, & A_{0}^{(\mathrm{l})} & =-M_{1}+\frac{\pi^{2}}{24} M_{1}-\frac{1}{2} M_{2}-\frac{1}{8} M_{3} .
\end{aligned}
$$

Here the notation was introduced for the integrals $M_{i}=M_{i}\left(x_{13}^{2} / x_{24}^{2}\right)$

$$
\begin{aligned}
& M_{1}=\int_{0}^{1} \frac{d \beta}{\beta-\bar{\alpha}} \ln \left(\frac{\bar{\alpha} \bar{\beta}}{\alpha \beta}\right)=-\frac{1}{2}\left[\pi^{2}+\ln ^{2}\left(\frac{x_{13}^{2}}{x_{24}^{2}}\right)\right], \\
& M_{2}=\int_{0}^{1} \frac{d \beta}{\beta-\bar{\alpha}} \ln \left(\frac{\bar{\alpha} \bar{\beta}}{\alpha \beta}\right) \ln (\beta \bar{\beta}), \\
& M_{3}=\int_{0}^{1} \frac{d \beta}{\beta-\bar{\alpha}} \ln \left(\frac{\bar{\alpha} \bar{\beta}}{\alpha \beta}\right) \ln ^{2}(\beta \bar{\beta}),
\end{aligned}
$$

with $\bar{\beta}=1-\beta, \bar{\alpha}=1-\alpha$ and $\bar{\alpha} / \alpha=x_{13}^{2} / x_{24}^{2}$. We do not need the explicit expressions for the integrals $M_{2}$ and $M_{3}$ since, as we will see shortly, the contributions proportional to $M_{2}$ and $M_{3}$ cancel in the sum of all diagrams (for completeness, their explicit form can be found in 
Appendix B.3). Note that the integrals (215) vanish in the forward limit $x_{13}^{2}=-x_{24}^{2}$.

We would like to stress that the above results were obtained in the Feynman gauge. Despite the fact that the contribution of each individual Feynman diagram to the light-like Wilson loop (or equivalently, the $A_{-n}^{(\alpha)}$-functions) is gauge-dependent, their sum is gauge-invariant.

Next, we substitute the obtained expressions for the coefficient functions, Eqs. (210) - (214), into (209) and finally arrive at the following remarkably simple expression for the two-loop correction,

$$
w^{(2)}=\left[\left(-x_{13}^{2} \mu^{2}\right)^{2 \epsilon}+\left(-x_{24}^{2} \mu^{2}\right)^{2 \epsilon}\right]\left\{\epsilon^{-2} \frac{\pi^{2}}{48}+\epsilon^{-1} \frac{7}{8} \zeta_{3}\right\}-\frac{\pi^{2}}{24} \ln ^{2}\left(\frac{x_{13}^{2}}{x_{24}^{2}}\right)-\frac{37}{720} \pi^{4}+O(\epsilon) .
$$

We verify that in the forward limit, i.e. for $x_{13}^{2}=-x_{24}^{2}$, this relation is in agreement with the previous calculations of Refs. [85, 190]. The following comments are in order.

Arriving at (216) we notice that the leading UV divergent $O\left(1 / \epsilon^{4}\right)$ and $O\left(1 / \epsilon^{3}\right)$ terms cancel in the sum of all diagrams, in agreement with (176). According to (212), the coefficients in front of $1 / \epsilon^{2}$ are given by a sum of a rational number and $\pi^{2}$-term. The rational terms cancel in the sum of all diagrams. As a consequence, the residue of the double pole in $\epsilon$ of $w^{(2)}$ in Eq. (216) is proportional to $\zeta_{2}$. In a similar manner, the residue of $w^{(2)}$ at the single pole in $\epsilon$ is proportional to $\zeta_{3}$ and this comes about as the result of a cancelation between various terms in (213) containing rational numbers, $\pi^{2}$-terms as well as the integrals $M_{1}$ and $M_{2}$. The most striking simplifications occur in the sum of finite $O\left(\epsilon^{0}\right)$ terms (214). We find that the integrals $M_{2}, M_{3}, M_{1}^{2}$ as well as the rational corrections and the terms proportional to $\pi^{2}$ and $\zeta_{3}$ cancel in the sum of all diagrams leading to $-\frac{7}{720} \pi^{4}+\frac{1}{12} \pi^{2} M_{1}$.

We would like to stress that the two-loop expression (216) satisfies the "maximal transcendentality principle" in $\mathcal{N}=4 \mathrm{SYM}\left[136\right.$. Let us assign transcendentality $n$ to functions $\mathrm{Li}_{n} 34$ and transcendentality 1 to a single pole $1 / \epsilon$. Then it is easy to see from (216) that the coefficient in front of $1 / \epsilon^{n}$ (including the finite $O\left(\epsilon^{0}\right)$ term) has transcendentality equal to $4-n$. In this way, each term in the two-loop expression (216) has transcendentality 4. For the same reason, the one-loop correction to the Wilson loop, Eq. (208) has transcendentality 2. Generalising this remarkable property to higher loops in planar $\mathcal{N}=4 \mathrm{SYM}$, we expect that the perturbative correction to the Wilson loop (167) to order $O\left(g^{2 n}\right)$ should have transcendentality $2 n$.

Notice that in our calculation of the two-loop Wilson loop we did not rely on the multicolour limit. In fact, due to the special form of the maximally non-Abelian colour factors, $c^{(n)}=C_{F} N^{n-1}$, relation (207) is exact for arbitrary $N$. As was already mentioned, these colour factors become more complicated starting from $n=4$ loops, where we should expect terms subleading in $N$.

\subsection{Pentagonal Wilson loop}

In [87], the calculation of the pentagonal Wilson loop at two loops was carried out in order to further test the duality (195). The Feynman diagrams are similar to those shown in Fig. 27. Individual diagrams can depend on ratios of the five Lorentz invariants available from five light-like distances, $x_{13}^{2}, x_{24}^{2}, x_{35}^{2}, x_{41}^{2}$ and $x_{52}^{2}$. We derived (multiple) parameter integral representations for all diagrams. The pole part of all diagrams could be evaluated analytically,

\footnotetext{
${ }^{34}$ E.g. $\ln (x)=\operatorname{Li}_{1}(1-x)$ and $\zeta_{2}=\pi^{2} / 6=\operatorname{Li}_{2}(1)$ are assigned transcendentality 1 and 2, respectively.
} 
and for the finite part we found within good numerical precision the following result:

$$
w_{5}^{(2)}=\left[\frac{1}{\epsilon^{2}} \frac{\pi^{2}}{96}+\frac{1}{\epsilon} \frac{7}{16} \zeta_{3}\right] \sum_{i=1}^{5}\left(-x_{i, i+2}^{2} \mu^{2}\right)^{2 \epsilon}+\frac{\pi^{2}}{48} \sum_{i=1}^{5} \ln \left(\frac{x_{i, i+2}^{2}}{x_{i, i+3}^{2}}\right) \ln \left(\frac{x_{i+1, i+3}^{2}}{x_{i+2, i+4}^{2}}\right)-\frac{\pi^{4}}{144} .
$$

We would like to mention that the calculation of the pole part in [87] was carried out analytically for arbitrary $n$ at two loops, in agreement with the general formula (176). As explained in that paper, one can define 'auxiliary' diagrams to subtract the pole terms, thereby providing a definition of the finite part of the two-loop $n$-cusp Wilson loop in terms of a set of finite parametric integrals.

\subsection{Check of the duality at two loops}

Let us introduce the following notation for the perturbative expansion of $\ln W\left(C_{n}\right)=Z_{n}+F_{n}^{(\mathrm{WL})}$ in the planar limit:

$$
F_{n}^{(\mathrm{WL})}=a F_{n ; 1}^{(\mathrm{WL})}+a^{2} F_{n ; 2}^{(\mathrm{WL})}+\ldots
$$

Then we see from (216) and (217) that we have

$$
\begin{aligned}
& F_{4 ; 2}^{(\mathrm{WL})}=-\frac{\pi^{2}}{24} \ln ^{2}\left(\frac{x_{13}^{2}}{x_{24}^{2}}\right)+\text { const }, \\
& F_{5 ; 2}^{(\mathrm{WL})}=\frac{\pi^{2}}{48} \sum_{i=1}^{5} \ln \left(\frac{x_{i, i+2}^{2}}{x_{i, i+3}^{2}}\right) \ln \left(\frac{x_{i+1, i+3}^{2}}{x_{i+2, i+4}^{2}}\right)+\text { const } .
\end{aligned}
$$

Comparing (219), (220) to (125), (126) it is straightforward to see that the duality relation (195) is verified for $n=4,5$ at two loops.

One may wonder, independently of the duality with gluon amplitudes, why the two-loop results for (the finite part of the logarithm of) the rectangular and pentagonal Wilson loops (219) and (220) are so simple. For example, one could have expected nontrivial multi-variable functions to appear in the 5-point case, such as harmonic polylogarithms. In section 8 we will see that the simplicity observed here is explained by conformal symmetry. There, we will derive all-order broken conformal Ward identities that have stringent implications for $F_{n}^{(\mathrm{WL})}$.

\section{Conformal symmetry of light-like Wilson loops}

In this section we review the derivation of all-order broken conformal Ward identities for the Wilson loops $W\left(C_{n}\right)$ [86, 87].

Recall that $C_{n}$ is a polygonal contour with $n$ light-like edges. The decisive observation is that such contours are stable under conformal transformations. That is, the contour $C_{n}^{\prime}$, which is the image of the contour $C_{n}$ upon a conformal transformation, is also made of $n$ light-like segments with new cusp points $x_{i}^{\prime \mu}$ obtained as the images of the old ones $x_{i}^{\mu}$. This property is rather obvious for translations, rotations and dilatations, but we have to check it for special conformal transformations. Performing a conformal inversion $35, x^{\mu \prime}=x^{\mu} / x^{2}$, of all points belonging to the segment

$$
x^{\mu}\left(\tau_{i}\right)=\tau_{i} x_{i}^{\mu}+\left(1-\tau_{i}\right) x_{i+1}^{\mu},
$$

with $\tau \in[0,1]$, we obtain another segment of the same type,

$$
x^{\prime \mu}\left(\tau_{i}^{\prime}\right)=\tau_{i}^{\prime} x_{i}^{\prime \mu}+\left(1-\tau_{i}^{\prime}\right) x_{i+1}^{\prime \mu},
$$

\footnotetext{
${ }^{35} \mathrm{~A}$ special conformal transformation (boost) is equivalent to an inversion followed by a translation and then another inversion (cf. section 2).
} 
with $\left(x_{i+1}^{\prime}-x_{i}^{\prime}\right)^{2}=0$ and $\tau_{i}^{\prime}=\tau_{i} /\left[\tau_{i}+\left(1-\tau_{i}\right)\left(x_{i}^{\prime}\right)^{2} /\left(x_{i+1}^{\prime}\right)^{2}\right]$.

Were the Wilson loop $W\left(C_{n}\right)$ well defined in $D=4$ dimensional Minkowski space-time, then it would enjoy the (super)conformal invariance of the underlying $\mathcal{N}=4 \mathrm{SYM}$ theory. More precisely, in the absence of conformal anomalies we would conclude that

$$
W\left(C_{n}^{\prime}\right)=W\left(C_{n}\right) .
$$

However, as we already pointed out in section 5.4, the light-like cusps of $C_{n}$ cause specific ultraviolet divergences to appear in the Wilson loop (167). For this reason we use dimensional regularisation with $D=4-2 \epsilon$ and $\epsilon>0$, which breaks the conformal invariance of the action, as we will see presently. In the dimensionally regularised $\mathcal{N}=4 \mathrm{SYM}$ theory, the Wilson loop $W\left(C_{n}\right) \equiv\left\langle W_{n}\right\rangle$ is given by a functional integral

$$
\left\langle W_{n}\right\rangle=\int \mathcal{D} A \mathcal{D} \lambda \mathcal{D} \phi \mathrm{e}^{i S_{\epsilon}[A, \lambda, \phi]} \operatorname{Tr}\left[\mathrm{P} \exp \left(i \oint_{C_{n}} d x \cdot A(x)\right)\right],
$$

where the integration goes over gauge fields, $A$, gaugino, $\lambda$, and scalars, $\phi$, with the action

$$
S_{\epsilon}=\frac{1}{g^{2} \mu^{2 \epsilon}} \int d^{D} x \mathcal{L}(x), \quad \mathcal{L}=\operatorname{Tr}\left[-\frac{1}{2} F_{\mu \nu}^{2}\right]+\text { gaugino }+ \text { scalars }+ \text { gauge fixing }+ \text { ghosts. }
$$

Here $\mu$ is the regularisation scale and we redefined all fields in such a way that $g$ does not appear inside the Lagrangian $\mathcal{L}(x)$. This allows us to keep the canonical dimension of all fields, in particular of the gauge field $A^{\mu}(x)$, and hence to preserve the conformal invariance of the path-ordered exponential entering the functional integral in (224). However, due to the change of dimension of the measure $\int d^{D} x$ in (225) the action $S_{\epsilon}$ is not invariant under dilatations and conformal boosts, which yields an anomalous contribution to the Ward identities.

\subsection{Anomalous conformal Ward identities}

The conformal Ward identities for the light-like Wilson loop $W\left(C_{n}\right)$ can be derived following the standard method [202, 134, 98, by acting on both sides of (225) with generators of conformal transformations.. The expressions of the generators of conformal transformations acting on fields are given in equation (17).

Let us start with the dilatations and perform a change of variables in the functional integral (224), $\phi_{I}^{\prime}(x)=\phi_{I}(x)+\varepsilon \mathbb{D} \phi_{I}(x)$. This change of variables could be compensated by a coordinate transformation $x^{\mu \prime}=(1-\varepsilon) x^{\mu}$. We recall that the path-ordered exponential is invariant under dilatations, whereas the Lagrangian is covariant with canonical weight $\Delta_{\mathcal{L}}=4$. However, the measure $\int d^{D} x$ with $D=4-2 \epsilon$ does not match the weight of the Lagrangian, which results in a non-vanishing variation of the action $S_{\epsilon}$,

$$
\delta_{\mathbb{D}} S_{\epsilon}=\frac{2 \epsilon}{g^{2} \mu^{2 \epsilon}} \int d^{D} x \mathcal{L}(x) .
$$

This variation generates an operator insertion into the expectation value, $\left\langle\delta_{\mathbb{D}} S_{\epsilon} W_{n}\right\rangle$, and yields an anomalous term in the action of the dilatation generator on $\left\langle W_{n}\right\rangle$, i.e.,

$$
\mathbb{D}\left\langle W_{n}\right\rangle=\sum_{i=1}^{n}\left(x_{i} \cdot \partial_{i}\right)\left\langle W_{n}\right\rangle=-\frac{2 i \epsilon}{g^{2} \mu^{2 \epsilon}} \int d^{D} x\left\langle\mathcal{L}(x) W_{n}\right\rangle .
$$

In a similar manner, the anomalous special conformal (or conformal boost) Ward identity is derived by performing transformations generated by the operator $\mathbb{K}^{\nu}$, Eq. (17), on both sides 
of (224). In this case the nonvanishing variation of the action $\delta_{\mathbb{K}^{\mu}} S_{\epsilon}$ again comes from the mismatch of the conformal weights of the Lagrangian and of the measure $\int d^{D} x$. 36 This amounts to considering just the $\Delta_{\phi}$ term in (17) with $\Delta_{\phi}=\Delta_{\mathcal{L}}-D=2 \epsilon$, and hence

$$
\mathbb{K}^{\nu}\left\langle W_{n}\right\rangle=\sum_{i=1}^{n}\left(2 x_{i}^{\nu} x_{i} \cdot \partial_{i}-x_{i}^{2} \partial_{i}^{\nu}\right)\left\langle W_{n}\right\rangle=-\frac{4 i \epsilon}{g^{2} \mu^{2 \epsilon}} \int d^{D} x x^{\nu}\left\langle\mathcal{L}(x) W_{n}\right\rangle .
$$

The relations (227) and (228) can be rewritten for $\ln \left\langle W_{n}\right\rangle$ as

$$
\begin{aligned}
& \mathbb{D} \ln \left\langle W_{n}\right\rangle=-\frac{2 i \epsilon}{g^{2} \mu^{2 \epsilon}} \int d^{D} x \frac{\left\langle\mathcal{L}(x) W_{n}\right\rangle}{\left\langle W_{n}\right\rangle}, \\
& \mathbb{K}^{\nu} \ln \left\langle W_{n}\right\rangle=-\frac{4 i \epsilon}{g^{2} \mu^{2 \epsilon}} \int d^{D} x x^{\nu} \frac{\left\langle\mathcal{L}(x) W_{n}\right\rangle}{\left\langle W_{n}\right\rangle} .
\end{aligned}
$$

To make use of these relations we have to evaluate the ratio $\left\langle\mathcal{L}(x) W_{n}\right\rangle /\left\langle W_{n}\right\rangle$ obtained by inserting the Lagrangian into the Wilson loop expectation value. Due to the presence of $\epsilon$ on the right-hand side of (229), it is sufficient to know its divergent part only.

\subsection{Dilatation Ward identity}

As we will now show, the dilatation Ward identity can be derived by dimensional arguments and this provides a consistency condition for the right-hand side of (229). By definition (224), the dimensionally regularised light-like Wilson loop $\left\langle W_{n}\right\rangle$ is a dimensionless scalar function of the cusp points $x_{i}^{\nu}$ and, as a consequence, it satisfies the relation

$$
\left(\sum_{i=1}^{n}\left(x_{i} \cdot \partial_{i}\right)-\mu \frac{\partial}{\partial \mu}\right) \ln \left\langle W_{n}\right\rangle=0 .
$$

In addition, its perturbative expansion is expressed in powers of the coupling $g^{2} \mu^{2 \epsilon}$ and, therefore,

$$
\mu \frac{\partial}{\partial \mu}\left\langle W_{n}\right\rangle=2 \epsilon g^{2} \frac{\partial}{\partial g^{2}}\left\langle W_{n}\right\rangle=-\frac{2 i \epsilon}{g^{2} \mu^{2 \epsilon}} \int d^{D} x\left\langle\mathcal{L}(x) W_{n}\right\rangle
$$

where the last relation follows from (224). Recall that according to (175), the Wilson loop $\left\langle W_{n}\right\rangle$ can be split into the product of divergent and finite parts

$$
\ln \left\langle W_{n}\right\rangle=Z_{n}+F_{n} .
$$

Notice that the definition of the divergent part is ambiguous as one can always add to $Z_{n}$ a term finite for $\epsilon \rightarrow 0$. Our definition (176) is similar to the conventional MS scheme with the only difference that we choose the expansion parameter to be $a \mu^{2 \epsilon}$ instead of $a$. The reason for this is that $Z_{n}$ satisfies, in our scheme, the same relation $\mu \partial_{\mu} Z_{n}=2 \epsilon g^{2} \partial_{g^{2}} Z_{n}$ as $\left\langle W_{n}\right\rangle$, see (231). Together with (232) and (230), this implies that the finite part of the Wilson loop does not depend on the renormalisation scale, i.e.

$$
\mu \partial_{\mu} F_{n}=O(\epsilon) .
$$

Using (232) and the explicit form of $Z_{n}$ in (176), the relation (231) leads to the following dilatation Ward identity 37

$$
\sum_{i=1}^{n}\left(x_{i} \cdot \partial_{x_{i}}\right) F_{n}=0 .
$$

\footnotetext{
${ }^{36}$ Another source of non-invariance of the action $S_{\epsilon}$ is the gauge-fixing term (and the associated ghost term of the non-Abelian theory) which is not conformally invariant even in four dimensions. However, due to gauge invariance of the Wilson loop, such anomalous terms do not appear on the right-hand side of (228).

${ }^{37}$ In what follows, we shall systematically neglect corrections to $F_{n}$ vanishing as $\epsilon \rightarrow 0$.
} 


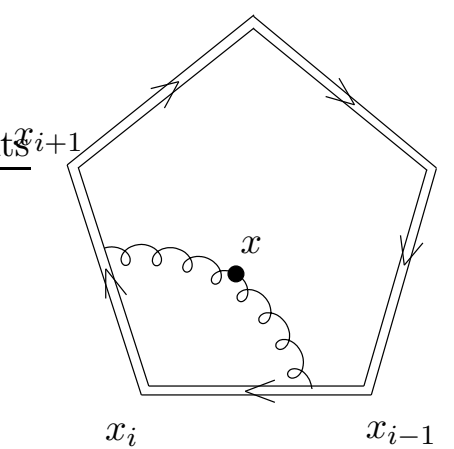

(a)

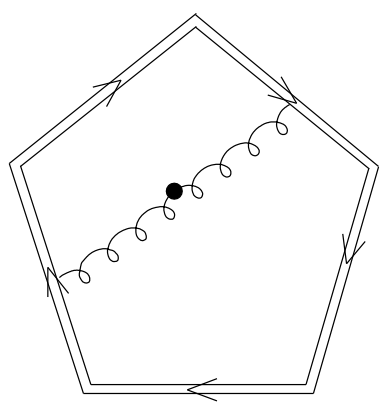

(b)

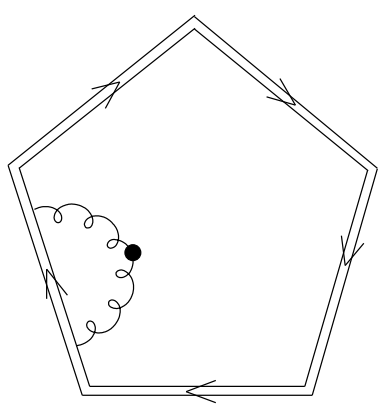

(c)

Figure 28: The Feynman diagrams contributing to $\left\langle\mathcal{L}(x) W_{n}\right\rangle$ to the lowest order in the coupling. The double line depicts the integration contour $C_{n}$, the wiggly line the gluon propagator and the blob the insertion point.

Adding to this the obvious requirement of Poincaré invariance, we conclude that the finite part $F_{n}$ of the light-like Wilson loop can depend on the dimensionless ratios $x_{i j}^{2} / x_{k l}^{2}$ only. In particular, for $n=4$ there is only one independent ratio, i.e. $F_{4}=F_{4}\left(x_{13}^{2} / x_{24}^{2}\right)$.

Furthermore, making use of (232), (176) and (234) we find that the all-loop dilatation Ward identity for $\left\langle W_{n}\right\rangle$ takes the form

$$
\mathbb{D} \ln \left\langle W_{n}\right\rangle=\sum_{i=1}^{n}\left(x_{i} \cdot \partial_{i}\right) \ln \left\langle W_{n}\right\rangle=-\frac{1}{2} \sum_{l \geq 1} a^{l} \sum_{i=1}^{n}\left(-x_{i-1, i+1}^{2} \mu^{2}\right)^{l \epsilon}\left(\frac{\Gamma_{\text {cusp }}^{(l)}}{l \epsilon}+\Gamma^{(l)}\right) .
$$

This relation provides a constraint on the form of the Lagrangian insertion on the right-hand side of (229).

\subsection{One-loop calculation of the anomaly}

The derivation of the dilatation Ward identity (234) relied on the known structure of cusp singularities (176) of the light-like Wilson loop and did not require a detailed knowledge of the properties of Lagrangian insertion $\left\langle\mathcal{L}(x) W_{n}\right\rangle /\left\langle W_{n}\right\rangle$. This is not the case anymore for the special conformal Ward identity.

To start with, let us perform an explicit one-loop computation of $\left\langle\mathcal{L}(x) W_{n}\right\rangle /\left\langle W_{n}\right\rangle$. To the lowest order in the coupling, we substitute $\left\langle W_{n}\right\rangle=1+O\left(g^{2}\right)$ in the denominator and retain inside $\mathcal{L}(x)$ and $W_{n}$ terms quadratic in gauge field only. The result is

$$
\frac{\left\langle\mathcal{L}(x) W_{n}\right\rangle}{\left\langle W_{n}\right\rangle}=-\frac{1}{4 N}\left\langle\operatorname{Tr}\left[\left(\partial_{\mu} A_{\nu}(x)-\partial_{\nu} A_{\mu}(x)\right)^{2}\right] \operatorname{Tr}\left[\left(i \oint_{C_{n}} d y \cdot A(y)\right)^{2}\right]\right\rangle+O\left(g^{6}\right) .
$$

The Wick contractions between gauge fields coming from the Lagrangian and the path-ordered exponential yield a product of two gluon propagators (170), each connecting the point $x$ with an arbitrary point $y$ on the integration contour $C_{n}$. Gauge invariance allows us to choose e.g. the Feynman gauge, in which the gluon propagator is given by (171). To the lowest order in the coupling constant, the right-hand side of (236) receives non-vanishing contributions from Feynman diagrams of three different topologies shown in Figs. 28(a) - (c).

In the Feynman gauge, only the vertex-like diagram shown in Fig. 28(a) develops poles in $\epsilon$. 
Performing the calculation, we find after some algebra 38

$$
\frac{2 i}{g^{2} \mu^{2 \epsilon}} \frac{\left\langle\mathcal{L}(x) W_{n}\right\rangle}{\left\langle W_{n}\right\rangle}=a \sum_{i=1}^{n}\left(-x_{i-1, i+1}^{2} \mu^{2}\right)^{\epsilon}\left\{\epsilon^{-2} \delta^{(D)}\left(x-x_{i}\right)+\epsilon^{-1} \Upsilon^{(1)}\left(x \mid x_{i-1}, x_{i}, x_{i+1}\right)+O\left(\epsilon^{0}\right)\right\},
$$

where $a=g^{2} N /\left(8 \pi^{2}\right)$ and the notation was introduced for

$$
\Upsilon^{(1)}\left(x \mid x_{i-1}, x_{i}, x_{i+1}\right)=\int_{0}^{1} \frac{d s}{s}\left[\delta^{(D)}\left(x-x_{i}-s x_{i-1, i}\right)+\delta^{(D)}\left(x-x_{i}+s x_{i, i+1}\right)-2 \delta^{(D)}\left(x-x_{i}\right)\right] .
$$

We see that the leading double-pole singularities are localised at the cusp points $x=x_{i}$. The subleading single poles are still localised on the contour, but they are 'smeared' along the lightlike edges adjacent to the cusp.

Substitution of (237) into (229) yields

$$
\begin{aligned}
& \mathbb{D} \ln \left\langle W_{n}\right\rangle=-a \sum_{i=1}^{n}\left(-x_{i-1, i+1}^{2} \mu^{2}\right)^{\epsilon} \epsilon^{-1}+O\left(a^{2}\right), \\
& \mathbb{K}^{\nu} \ln \left\langle W_{n}\right\rangle=-2 a \sum_{i=1}^{n} x_{i}^{\nu}\left(-x_{i-1, i+1}^{2} \mu^{2}\right)^{\epsilon} \epsilon^{-1}+O\left(a^{2}\right) .
\end{aligned}
$$

Notice that $\sum_{i=1}^{n} \Upsilon^{(1)}\left(x \mid x_{i-1}, x_{i}, x_{i+1}\right)$ does not contribute to the right-hand sides of these relations by virtue of

$$
\begin{aligned}
& \int d^{D} x \Upsilon^{(1)}\left(x \mid x_{i-1}, x_{i}, x_{i+1}\right)=0, \\
& \int d^{D} x x^{\nu} \Upsilon^{(1)}\left(x \mid x_{i-1}, x_{i}, x_{i+1}\right)=\left(x_{i-1}+x_{i+1}-2 x_{i}\right)^{\nu} .
\end{aligned}
$$

As was already mentioned, the right-hand sides of the Ward identities (239) are different from zero due to the fact that the light-like Wilson loop has cusp singularities.

We verify with the help of (177) that to the lowest order in the coupling, the first relation in (239) is in agreement with (235).

\subsection{Structure of the anomaly to all loops}

To extend the analysis of the special conformal Ward identity to all loops we examine the allloop structure of divergences of $\left\langle\mathcal{L}(x) W_{n}\right\rangle /\left\langle W_{n}\right\rangle$. They arise in a very similar way to those of the Wilson loop itself, which were discussed in section 5.4.2.

It is convenient to couple $\mathcal{L}(x)$ to an auxiliary 'source' $J(x)$ and rewrite the insertion of the Lagrangian into $\left\langle W_{n}\right\rangle$ as a functional derivative

$$
\left\langle\mathcal{L}(x) W_{n}\right\rangle /\left\langle W_{n}\right\rangle=-\left.i \frac{\delta}{\delta J(x)} \ln \left\langle W_{n}\right\rangle_{J}\right|_{J=0}
$$

where the subscript $J$ indicates that the expectation value is taken in the $\mathcal{N}=4 \mathrm{SYM}$ theory with the additional term $\int d^{D} x J(x) \mathcal{L}(x)$ added to the action. This generates new interaction

\footnotetext{
${ }^{38}$ It is advantageous to perform the calculation by taking a Fourier transform with respect to $x$ and later take the inverse transform of the final result.
} 
vertices inside Feynman diagrams for $\left\langle W_{n}\right\rangle_{J}$, but does not affect the non-Abelian exponentiation property (182) 39 The only difference compared to (182) is that the webs $w^{(k)}$ now depend on $J(x)$ through a new interaction vertex. Making use of the non-Abelian exponentiation we obtain

$$
\left\langle\mathcal{L}(x) W_{n}\right\rangle /\left\langle W_{n}\right\rangle=\left.\sum_{k=1}^{\infty}\left(\frac{g^{2}}{4 \pi^{2}}\right)^{k} c^{(k)}\left[-i \frac{\delta w^{(k)}}{\delta J(x)}\right]\right|_{J=0} .
$$

As for $\ln \left\langle W_{n}\right\rangle$, the webs produce at most double poles in $\epsilon$, and are localised at a given cusp. This allows us to write down a general expression for the divergent part of the Lagrangian insertion,

$$
\begin{aligned}
\frac{2 i \epsilon}{g^{2} \mu^{2 \epsilon}} \frac{\left\langle\mathcal{L}(x) W_{n}\right\rangle}{\left\langle W_{n}\right\rangle}= & \sum_{l \geq 1} a^{l} \sum_{i=1}^{n}\left(-x_{i-1, i+1}^{2} \mu^{2}\right)^{l \epsilon} \times \\
& \left\{\frac{1}{2}\left(\frac{\Gamma_{\text {cusp }}^{(l)}}{l \epsilon}+\Gamma^{(l)}\right) \delta^{(D)}\left(x-x_{i}\right)+\Upsilon^{(l)}\left(x ; x_{i-1}, x_{i}, x_{i+1}\right)\right\}+O(\epsilon),
\end{aligned}
$$

which generalises (237) to all loops. The following comments are in order.

In Eq. (243), the term proportional to $\delta^{(D)}\left(x-x_{i}\right)$ comes from the double pole contribution to the web $w^{(k)}$, which is indeed located at short distances in the vicinity of the cusp $x_{i}^{\mu}$. The residue of the simple pole in the right-hand side of (243) is given by the cusp anomalous dimension. This can be shown by substituting (243) into the dilatation Ward identity (227) and comparing with (235).

The contact nature of the leading singularity in (243) can also be understood in the following way. The correlator on the left-hand side can be viewed as a conformal $(n+1)$-point function with the Lagrangian at one point and the rest corresponding to the cusps. In the $\mathcal{N}=4 \mathrm{SYM}$ theory the Lagrangian belongs to the protected stress-tensor multiplet, therefore it has a fixed conformal dimension four. The Wilson loop itself, if it were not divergent, would be conformally invariant. This means that the $n$ cusp points can be regarded as having vanishing conformal weights. Of course, the presence of divergences might make the conformal properties anomalous. However, the conformal behaviour of the leading singularity in (243) cannot be corrected by an anomaly 40 Then we can argue that the only function of space-time points, which has conformal weight four at one point and zero at all other points, is the linear combination of delta functions appearing in (243) 41

As was already emphasised, the residue of the simple pole of $\delta w^{(k)} / \delta J(x)$ at the cusp point $x_{i}$ depends on its position and at most on its two nearest neighbours $x_{i-1}$ and $x_{i+1}$, as well as on the insertion point. It gives rise to a function $\Upsilon^{(l)}\left(x ; x_{i-1}, x_{i}, x_{i+1}\right)$, which is the same for all cusp points due to the cyclic symmetry of the Wilson loop.

Notice that in (243) we have chosen to separate the terms with the collinear anomalous dimension $\Gamma^{(l)}$ from the rest of the finite terms. This choice has implications for the function $\Upsilon^{(l)}\left(x ; x_{i-1}, x_{i}, x_{i+1}\right)$. Substituting the known factor $Z_{n}(176)$ and the particular form of (243)

\footnotetext{
${ }^{39}$ We recall that the non-Abelian exponentiation is not sensitive to the form of the action.

${ }^{40} \mathrm{Such}$ an anomalous contribution should come from a $1 / \epsilon^{3}$ pole in the correlator with two insertions of the Lagrangian, but repeated use of the argument above shows that the order of the poles does not increase with the number of insertions.

${ }^{41}$ The mismatch of the conformal weight four of the Lagrangian and $D=4-2 \epsilon$ of the delta functions does not affect the leading singularity in (243).
} 
into the dilatation Ward identity (227), we derive

$$
\sum_{i=1}^{n} \int d^{D} x \Upsilon^{(l)}\left(x ; x_{i-1}, x_{i}, x_{i+1}\right)=0 .
$$

We can argue that in fact each term in this sum vanishes. Indeed, each term in the sum is a Poincaré invariant dimensionless function of three points $x_{i-1}, x_{i}$ and $x_{i+1}$. Given the lightlike separation of the neighbouring points, the only available invariant is $x_{i-1, i+1}^{2}$. Further, $\Upsilon^{(l)}\left(x ; x_{i-1}, x_{i}, x_{i+1}\right)$ cannot depend on the regularisation scale because $\mu$ always comes in the combination $a \mu^{2 \epsilon}$ and thus contributes to the $O(\epsilon)$ terms in (243). The dimensionless Poincaré invariant $\int d^{D} x \Upsilon^{(l)}\left(x ; x_{i-1}, x_{i}, x_{i+1}\right)$ depends on a single scale and, therefore, it must be a constant, after which (244) implies

$$
\int d^{D} x \Upsilon^{(l)}\left(x ; x_{i-1}, x_{i}, x_{i+1}\right)=0
$$

For $l=1$ this relation is in agreement with the one-loop result (240).

\subsection{Special conformal Ward identity}

We are now ready to investigate the special conformal Ward identity (228). Inserting (243) into its right-hand side, and integrating over $x$ we obtain

$$
\begin{aligned}
\mathbb{K}^{\nu} \ln W_{n} & =\sum_{i=1}^{n}\left(2 x_{i}^{\nu} x_{i} \cdot \partial_{i}-x_{i}^{2} \partial_{i}^{\nu}\right) \ln W_{n} \\
& =-\sum_{l \geq 1} a^{l}\left(\frac{\Gamma_{\text {cusp }}^{(l)}}{l \epsilon}+\Gamma^{(l)}\right) \sum_{i=1}^{n}\left(-x_{i-1, i+1}^{2} \mu^{2}\right)^{l \epsilon} x_{i}^{\nu}-2 \sum_{i=1}^{n} \Upsilon^{\nu}\left(x_{i-1}, x_{i}, x_{i+1}\right)+O(\epsilon)
\end{aligned}
$$

where

$$
\Upsilon^{\nu}\left(x_{i-1}, x_{i}, x_{i+1}\right)=\sum_{l \geq 1} a^{l} \int d^{D} x x^{\nu} \Upsilon^{(l)}\left(x ; x_{i-1}, x_{i}, x_{i+1}\right) .
$$

Next, we substitute $\ln W_{n}=Z_{n}+F_{n}$ into (246), replace $\ln Z_{n}$ by its explicit form (176) and expand the right-hand side in powers of $\epsilon$ to rewrite (246) as follows:

$$
\mathbb{K}^{\nu} F_{n}=\frac{1}{2} \Gamma_{\text {cusp }}(a) \sum_{i=1}^{n} \ln \frac{x_{i, i+2}^{2}}{x_{i-1, i+1}^{2}} x_{i, i+1}^{\nu}-2 \sum_{i=1}^{n} \Upsilon^{\nu}\left(x_{i-1}, x_{i}, x_{i+1}\right)+O(\epsilon) .
$$

Note that the quantities $\Upsilon^{\nu}\left(x_{i-1}, x_{i}, x_{i+1}\right)$ are translation invariant. Indeed, a translation under the integral in (247) only affects the factor $x^{\nu}$ (the functions $\Upsilon^{(l)}\left(x ; x_{i-1}, x_{i}, x_{i+1}\right)$ are translation invariant), but the result vanishes as a consequence of (245). Furthermore, $\Upsilon^{\nu}\left(x_{i-1}, x_{i}, x_{i+1}\right)$ only depends on two neighbouring light-like vectors $x_{i-1, i}^{\mu}$ and $x_{i, i+1}^{\mu}$, from which we can form only one non-vanishing Poincaré invariant, $x_{i-1, i+1}^{2}$. We have already argued that $\Upsilon^{(l)}$ are independent of $\mu$, and so must be $\Upsilon^{\nu}$. Taking into account the scaling dimension one of $\Upsilon^{\nu}$, we conclude that

$$
\Upsilon^{\nu}\left(x_{i-1}, x_{i}, x_{i+1}\right)=\alpha x_{i-1, i}^{\nu}+\beta x_{i, i+1}^{\nu},
$$

where $\alpha, \beta$ only depend on the coupling. The symmetry of the Wilson loop $W_{n}$ under mirror exchange of the cusp points translates into symmetry of (249) under exchange of the neighbours $x_{i-1}$ and $x_{i+1}$ of the cusp point $x_{i}$, which reduces (249) to

$$
\Upsilon^{\nu}\left(x_{i-1}, x_{i}, x_{i+1}\right)=\alpha\left(x_{i-1}^{\nu}+x_{i+1}^{\nu}-2 x_{i}^{\nu}\right),
$$


with $\alpha=a+O\left(a^{2}\right)$ according to (240). Substituting this relation into (248) we find

$$
\sum_{i=1}^{n} \Upsilon^{\nu}\left(x_{i-1}, x_{i}, x_{i+1}\right)=0
$$

This concludes the derivation of the special conformal Ward identity. In the limit $\epsilon \rightarrow 0$ it takes the form (cf. [86])

$$
\sum_{i=1}^{n}\left(2 x_{i}^{\nu} x_{i} \cdot \partial_{i}-x_{i}^{2} \partial_{i}^{\nu}\right) F_{n}=\frac{1}{2} \Gamma_{\text {cusp }}(a) \sum_{i=1}^{n} \ln \frac{x_{i, i+2}^{2}}{x_{i-1, i+1}^{2}} x_{i, i+1}^{\nu} .
$$

\subsection{Solution and implications for $F_{n}$}

Let us now examine the consequences of the conformal Ward identity (252) for the finite part of the Wilson loop $W_{n}$. We find that the cases of $n=4$ and $n=5$ are special because here the Ward identity (252) has a unique solution up to an additive constant. The solutions are, respectively,

$$
\begin{aligned}
& F_{4}=\frac{1}{4} \Gamma_{\text {cusp }}(a) \ln ^{2}\left(\frac{x_{13}^{2}}{x_{24}^{2}}\right)+\text { const }, \\
& F_{5}=-\frac{1}{8} \Gamma_{\text {cusp }}(a) \sum_{i=1}^{5} \ln \left(\frac{x_{i, i+2}^{2}}{x_{i, i+3}^{2}}\right) \ln \left(\frac{x_{i+1, i+3}^{2}}{x_{i+2, i+4}^{2}}\right)+\text { const },
\end{aligned}
$$

as can be easily verified by making use of the identity

$$
\mathbb{K}^{\mu} x_{i j}^{2}=2\left(x_{i}^{\mu}+x_{j}^{\mu}\right) x_{i j}^{2} .
$$

We find that, upon identification of the kinematical invariants

$$
x_{k, k+r}^{2}:=\left(p_{k}+\ldots+p_{k+r-1}\right)^{2},
$$

the relations (253) and (254) are exactly the functional forms of the ansatz of [39] for the finite parts of the four- and five-point MHV amplitudes (or rather the ratio of the amplitude to the corresponding tree amplitude).

The reason why the functional form of $F_{4}$ and $F_{5}$ is fixed up to an additive constant is that there are no conformal invariants one can build from four or five points $x_{i}$ with light-like separations $x_{i, i+1}^{2}=0$. Such invariants take the form of cross-ratios

$$
\frac{x_{i j}^{2} x_{k l}^{2}}{x_{i k}^{2} x_{j l}^{2}}
$$

It is obvious that with four or five points they cannot be constructed. This becomes possible starting from six points, where there are three such cross-ratios, e.g.,

$$
u_{1}=\frac{x_{13}^{2} x_{46}^{2}}{x_{14}^{2} x_{36}^{2}}, \quad u_{2}=\frac{x_{24}^{2} x_{15}^{2}}{x_{25}^{2} x_{14}^{2}}, \quad u_{3}=\frac{x_{35}^{2} x_{26}^{2}}{x_{36}^{2} x_{25}^{2}} .
$$

Hence the general solution of the Ward identity at six cusp points and higher will contain an arbitrary function of the conformal cross-ratios.

Nevertheless, one can prove that the functional form of the ansatz of [39] still provides a particular solution to the Ward identity (252) [86]. The ansatz of [39] for the logarithm of the ratio of the amplitude to the tree amplitude reads

$$
\ln \mathcal{M}_{n}^{(\mathrm{MHV})}=Z_{n}+F_{n}^{(\mathrm{BDS})}+C_{n}+O(\epsilon),
$$


and

$$
F_{n}^{(\mathrm{BDS})}=\frac{1}{2} \Gamma_{\text {cusp }}(a) \mathcal{F}_{n}
$$

where $Z_{n}$ is the IR divergent part, $F_{n}$ is the finite part depending on the Mandelstam variables and $C_{n}$ is the constant term. At four points the proposed form of the finite part, written in the dual notation (196), is

$$
\mathcal{F}_{4}=\frac{1}{2} \ln ^{2}\left(\frac{x_{13}^{2}}{x_{24}^{2}}\right)+4 \zeta_{2}
$$

while for $n \geq 5$ it is

$$
\mathcal{F}_{n}=\frac{1}{2} \sum_{i=1}^{n} g_{n, i}, \quad g_{n, i}=-\sum_{r=2}^{\left\lfloor\frac{n}{2}\right\rfloor-1} \ln \left(\frac{x_{i, i+r}^{2}}{x_{i, i+r+1}^{2}}\right) \ln \left(\frac{x_{i+1, i+r+1}^{2}}{x_{i, i+r+1}^{2}}\right)+D_{n, i}+L_{n, i}+\frac{3}{2} \zeta_{2} .
$$

The functions $D_{n, i}$ and $L_{n, i}$ depend on whether $n$ is odd or even. For $n$ odd, $n=2 m+1$, they are

$$
\begin{aligned}
D_{n, i} & =-\sum_{r=2}^{m-1} \operatorname{Li}_{2}\left(1-\frac{x_{i, i+r}^{2} x_{i-1, i+r+1}^{2}}{x_{i, i+r+1}^{2} x_{i-1, i+r}^{2}}\right), \\
L_{n, i} & =-\frac{1}{2} \ln \left(\frac{x_{i, i+m}^{2}}{x_{i, i+m+1}^{2}}\right) \ln \left(\frac{x_{i+1, i+m+1}^{2}}{x_{i+m, i+2 m}^{2}}\right) .
\end{aligned}
$$

For $n$ even, $n=2 m$, they are

$$
\begin{aligned}
D_{n, i} & =-\sum_{r=2}^{m-2} \operatorname{Li}_{2}\left(1-\frac{x_{i, i+r}^{2} x_{i-1, i+r+1}^{2}}{x_{i, i+r+1}^{2} x_{i-1, i+r}^{2}}\right)-\frac{1}{2} \operatorname{Li}_{2}\left(1-\frac{x_{i, i+m-1}^{2} x_{i-1, i+m}^{2}}{x_{i, i+m}^{2} x_{i-1, i+m-1}^{2}}\right), \\
L_{n, i} & =\frac{1}{4} \ln ^{2}\left(\frac{x_{i, i+m}^{2}}{x_{i+1, i+m+1}^{2}}\right) .
\end{aligned}
$$

We have already seen that at four points and five points the general solution to the Ward identity coincides with (259). We now show that the ansatz (259) is a solution of the Ward identity for arbitrary $n$.

First we observe that the dilogarithmic contributions in (263) and (264) are functions of conformal cross-ratios of the form (257). They are therefore invariant under conformal transformations and we have immediately

$$
K^{\mu} D_{n, i}=0 .
$$

For the logarithmic contributions we use the identity (255). When $n$ is odd we then find

$$
\begin{gathered}
K^{\mu} g_{n, i}=-2 \sum_{r=2}^{m-1}\left[x_{i+r, i+r+1}^{\mu}\left(\ln x_{i+1, i+r+1}^{2}-\ln x_{i, i+r+1}^{2}\right)-x_{i, i+1}^{\mu}\left(\ln x_{i, i+r}^{2}-\ln x_{i, i+r, 2}^{2}\right)\right] \\
-x_{i+m, i+m+1}^{\mu}\left(\ln x_{i+1, i+m+1}^{2}-\ln x_{i+m, i+2 m}^{2}\right)-\left(x_{i+1, i+2 m}^{\mu}-x_{i+m, i+m+1}^{\mu}\right)\left(\ln x_{i, i+m}^{2}-\ln x_{i+m+1, i}^{2}\right) .
\end{gathered}
$$

Changing variables term by term in the sum over $i$ one finds that only the $\ln x_{i, i+2}^{2}$ terms remain and indeed (252) is satisfied. The proof for $n$ even goes exactly the same way except that one obtains

$$
K^{\mu} \mathcal{F}_{n}=\sum_{i=1}^{n}\left[\ln x_{i, i+2}^{2}\left(x_{i}^{\mu}+x_{i+2}^{\mu}-2 x_{i+1}^{\mu}\right)+\frac{1}{2} \ln x_{i, i+m}^{2}\left(x_{i+m-1, i+m+1}^{\mu}-x_{i-1, i+1}^{\mu}\right)\right]
$$

and one has to use the fact that $n=2 m$ to see that the $\ln x_{i, i+m}^{2}$ term vanishes under the sum.

Thus we have seen that the BDS ansatz for the $n$-gluon MHV amplitudes satisfies the conformal Ward identity for the Wilson loop. 


\section{Hexagon Wilson loop and six-gluon MHV amplitude}

In section 7 we saw that the duality (5) is valid at two loops for $n=4,5$ points (or gluons). For $n=4,5$, the Ward identities derived in section 8 for the Wilson loops provide a possible explanation of the duality to all orders in the coupling constant, assuming the BDS ansatz is correct in these cases. This speculation receives support from strong coupling, where the result of [79] is in agreement with the BDS ansatz for $n=4$.

Recall that the authors of [80] found a disagreement with the BDS ansatz at strong coupling for the number of gluons $n$ very large. Given this result it seems natural to ask whether the ansatz already breaks down at some perturbative level and for a finite number of gluons. If we assume the duality with Wilson loops, then $n=4$ and $n=5$ are completely fixed by conformal symmetry. In fact, one might even suspect that the observed validity of the duality relation for $n=4,5$ is true only because both objects have the same conformal symmetry. To put it differently, the proposed duality might be reduced to the weaker (but still highly non-trivial) statement that the MHV gluon amplitudes are governed by dual conformal symmetry. The first real test of the stronger form of the duality (5) is provided by the case $n=6$. In order to distinguish between these possibilities we performed a two-loop calculation of the hexagonal Wilson loop. 42

\subsection{Finite part of the hexagon Wilson loop}

The finite part of the hexagon Wilson loop, $F_{6}^{(\mathrm{WL})}$, does not depend on the renormalisation scale and it is a dimensionless function of the distances $x_{i j}^{2}$. Since the edges of $C_{6}$ are light-like, $x_{i, i+1}^{2}=0$, the only nonzero distances are $x_{i, i+2}^{2}$ and $x_{i, i+3}^{2}$ (with $i=1, \ldots, 6$ and the periodicity condition $x_{i+6}=x_{i}$ ). We saw in section 8 that it has to satisfy the conformal Ward identity (252). Specified to $n=6$, its general solution is given by [86]

$$
F_{6}^{(\mathrm{WL})}=F_{6}^{(\mathrm{BDS})}+R_{6}\left(u_{1}, u_{2}, u_{3} ; a\right) .
$$

Here, upon the identification $p_{i}=x_{i+1}-x_{i}$,

$$
\begin{aligned}
F_{6}^{(\mathrm{BDS})}=\frac{1}{4} \Gamma_{\text {cusp }}(a) \sum_{i=1}^{6} & {\left[-\ln \left(\frac{x_{i, i+2}^{2}}{x_{i, i+3}^{2}}\right) \ln \left(\frac{x_{i+1, i+3}^{2}}{x_{i, i+3}^{2}}\right)\right.} \\
+ & \left.\frac{1}{4} \ln ^{2}\left(\frac{x_{i, i+3}^{2}}{x_{i+1, i+4}^{2}}\right)-\frac{1}{2} \operatorname{Li}_{2}\left(1-\frac{x_{i, i+2}^{2} x_{i+3, i+5}^{2}}{x_{i, i+3}^{2} x_{i+2, i+5}^{2}}\right)\right]
\end{aligned}
$$

while $R_{6}\left(u_{1}, u_{2}, u_{3} ; a\right)$ is an arbitrary function of the three cross-ratios 43

$$
u_{1}=\frac{x_{13}^{2} x_{46}^{2}}{x_{14}^{2} x_{36}^{2}}, \quad u_{2}=\frac{x_{24}^{2} x_{15}^{2}}{x_{25}^{2} x_{14}^{2}}, \quad u_{3}=\frac{x_{35}^{2} x_{26}^{2}}{x_{36}^{2} x_{25}^{2}} .
$$

These variables are invariant under conformal transformations of the coordinates $x_{i}^{\mu}$, and therefore they are annihilated by the conformal boost operator entering the left-hand side of (252). In addition, the Wilson loop $W\left(C_{6}\right)$ is invariant under cyclic $\left(x_{i} \rightarrow x_{i+1}\right)$ and mirror $\left(x_{i} \rightarrow x_{6-i}\right)$ permutations of the cusp points [87]. This implies that $R\left(u_{1}, u_{2}, u_{3}\right)$ is a totally symmetric function of three variables.

\footnotetext{
${ }^{42}$ This section is based on the publications 90 and 91 .

${ }^{43}$ The last term in (269) is a function of cross-ratios only, but we keep it in $F_{6}^{(\mathrm{BDS})}$, because it is part of the BDS conjecture.
} 
The one-loop Wilson loop calculation of [84 has shown that the 'remainder' function $R_{6}$ is just a constant at one loop, which is a confirmation of the Wilson loop/scattering amplitude duality going beyond the scope of conformal symmetry. However, one might suspect just a low loop-order 'accident'. The point is that the function $R_{6}$ must satisfy a further, rather powerful constraint, coming from the collinear limit of gluon amplitudes [52, 56]. It could be that due to the limited choice of loop integrals at this low perturbative level, the function (269) made of them is the only one satisfying both the conformal Ward identity (252) and the collinear limit. If so, at some higher perturbative level new functions with these properties might appear which could spoil the BDS ansatz and/or the Wilson loop/scattering amplitude duality.

Combining together (195) and (268), we conclude that were the BDS conjecture and the duality relation (195) correct for $n=6$, we would expect that $R\left(u_{1}, u_{2}, u_{3}\right)=$ const. The explicit two-loop calculation we report on here shows that this is not true.

For the sake of simplicity we performed the calculation of $W\left(C_{6}\right)$ in the Feynman gauge. In addition, we made use of the non-Abelian exponentiation property of Wilson loops [176, 194, 195] to reduce the number of relevant Feynman diagrams. In application to $R\left(u_{1}, u_{2}, u_{3}\right)$ this property can be formulated as follows (the same property also holds for $F_{6}^{(\mathrm{WL})}$ )

$$
R=\frac{g^{2}}{4 \pi^{2}} C_{F} R^{(1)}+\left(\frac{g^{2}}{4 \pi^{2}}\right)^{2} C_{F} N R^{(2)}+O\left(g^{6}\right)
$$

where $C_{F}=\left(N^{2}-1\right) /(2 N)$ is the Casimir in the fundamental representation of $S U(N)$. The functions $R^{(1)}$ and $R^{(2)}$ do not involve the colour factors and only depend on the distances between the cusp points on $C_{6}$. At one loop, $R^{(1)}\left(u_{1}, u_{2}, u_{3}\right)$ is in fact a constant [84].

As explained in [86], the relation (271) implies that in order to determine the function $F_{6}^{(\mathrm{WL})}$ at two loops (and hence $f^{(2)}$ ) it is sufficient to calculate the contribution to $W\left(C_{6}\right)$ from two-loop diagrams containing the 'maximally non-Abelian' colour factor $C_{F} N$ only. All relevant two-loop graphs are shown in Fig. 29, The finite part of $W\left(C_{6}\right)$ receives contributions from all Feynman
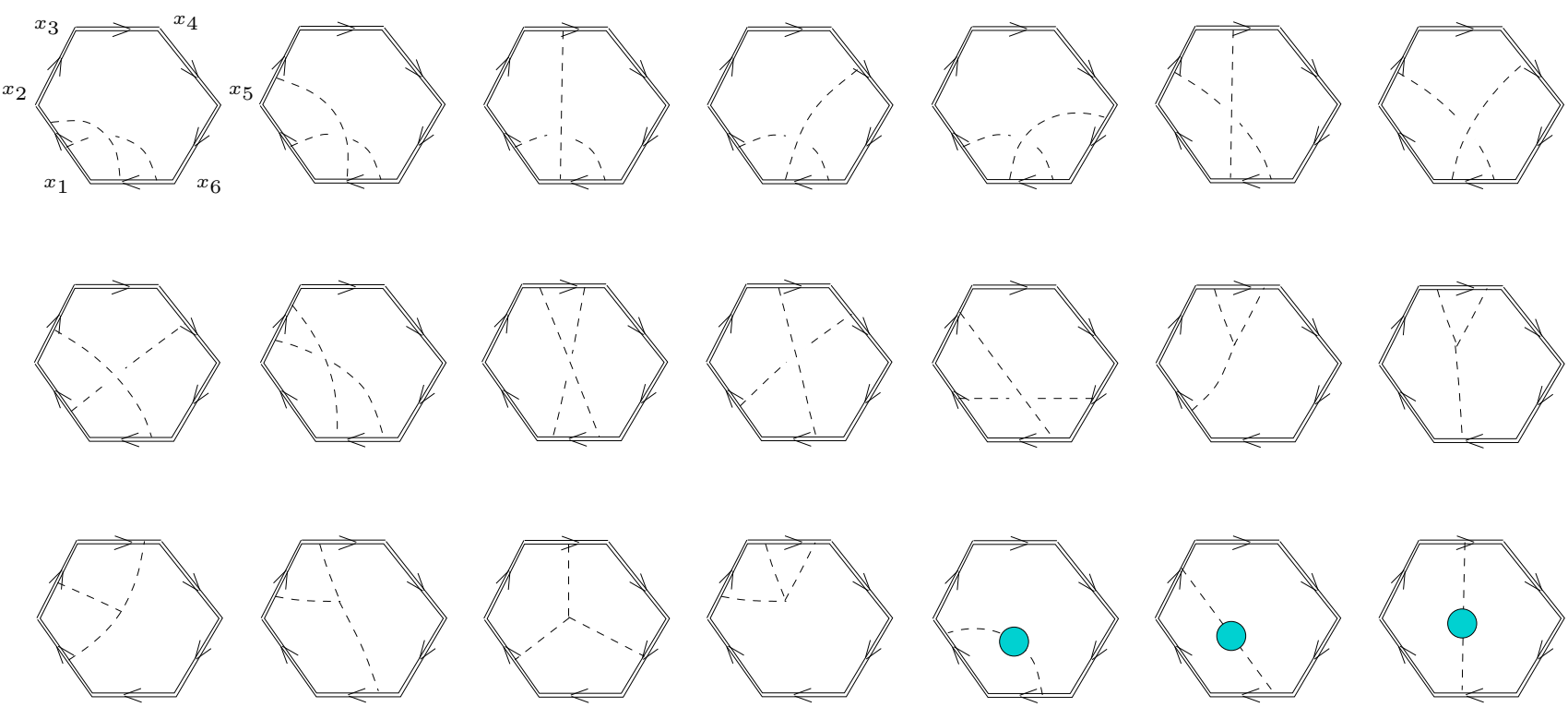

Figure 29: The maximally non-Abelian Feynman diagrams of different topology contributing to $F_{6}^{(\mathrm{WL})}$. The double lines depict the integration contour $C_{6}$, the dashed lines depict the gluon propagator and the blob stands for the one-loop polarisation operator. 
diagrams shown in Fig. 29, We derived parameter integral representations for them, which are denoted by $A_{0}^{(\alpha)}$, so that the two-loop contribution to $F_{6}^{(\mathrm{WL})}=\sum_{p \geq 1} a^{p} F_{6 ; p}^{(\mathrm{WL})}$ is given by

$$
F_{6 ; 2}^{(\mathrm{WL})}=2 \sum_{\alpha=\mathrm{a}, \ldots, \mathrm{u}} A_{0}^{(\alpha)} .
$$

The integrals $A_{0}^{(\alpha)}$ are difficult to compute analytically and so we evaluated them numerically, as described in the next section.

\subsection{Numerical evaluation}

For a given set of kinematical invariants,

$$
K=\left\{x_{13}^{2}, x_{14}^{2}, x_{15}^{2}, x_{24}^{2}, x_{25}^{2}, x_{35}^{2}, x_{26}^{2}, x_{36}^{2}, x_{46}^{2}\right\},
$$

defined as the distances between the vertices of the hexagon $C_{6}$, it is straightforward to evaluate the sum on the right-hand side of (272) numerically. We should take into account, however, that in $D=4$ dimensions there exists a relationship between the distances (273), such that only eight of them are independent. This relation reflects the fact that the five four-dimensional vectors $p_{i}^{\mu}=x_{i+1}^{\mu}-x_{i}^{\mu}($ with $i=1, \ldots, 5)$ are linearly dependent, and hence their Gram determinant vanishes

$$
G[K]=\operatorname{det}\left\|\left(p_{i} \cdot p_{j}\right)\right\|=0, \quad(i, j=1, \ldots, 5) .
$$

Using $p_{i}=x_{i+1}-x_{i}$, the entries of the matrix can be written as linear combinations of the distances (273).

We would like to note that the sum on the right-hand side of (272) defines a function of $x_{i j}^{2}$ even for configurations $K$ which do not satisfy (274). This function can be viewed as a particular continuation of the finite part of the hexagon Wilson loop off the hypersurface defined by (274).

Let us now consider the relation between the hexagon Wilson loop and the BDS ansatz, Eq. (268). To two-loop accuracy it takes the form

$$
R_{\mathrm{W}}\left(u_{1}, u_{2}, u_{3}\right)=F_{6 ; 2}^{(\mathrm{WL})}-F_{6 ; 2}^{(\mathrm{BDS})},
$$

where $R_{\mathrm{W}}$ and $F_{6 ; 2}^{(\mathrm{BDS})}$ denote the two-loop contributions to the remainder function (271) and to the BDS ansatz (269)), respectively. We recall that the functions $F_{6 ; 2}^{(\mathrm{WL})}$ and $F_{6 ; 2}^{(\mathrm{BDS})}$ satisfy the Ward identity (228), and hence $R_{\mathrm{W}}$ is a function of the three conformal cross-ratios (270) only.

The simplest way to check relation (275) is to evaluate the difference $F_{6 ; 2}^{(\mathrm{WL})}-F_{6 ; 2}^{(\mathrm{BDS})}$ for different kinematical configurations $K$ and $K^{\prime}$, related to each other by a conformal transformation of the coordinates $x_{i}^{\mu}$ (with $i=1, \ldots, 6$ ). Since $F_{6 ; 2}^{(\mathrm{WL})}$ and $F_{6 ; 2}^{(\mathrm{BDS})}$ are dimensionless functions of the distances $x_{i j}^{2}$, they are automatically invariant under translations, Lorentz rotations and dilatations of the coordinates $x_{i}^{\mu}$. The only non-trivial transformations are the special conformal transformations (boosts), which are combinations of an inversion, a translation and another inversion.

Let us start with a kinematical configuration $K=K\left(x_{i j}^{2}\right)$, Eq. (273), and perform an inversion of the coordinates, $x_{i}^{\mu} \rightarrow x_{i}^{\mu} / x_{i}^{2}$, to define the new configuration

$$
K^{\prime}=K\left(x_{i j}^{2} /\left(x_{i}^{2} x_{j}^{2}\right)\right) .
$$


Since the variables $u(270)$ are invariant under such transformations, $u_{a}[K]=u_{a}\left[K^{\prime}\right]$, the difference $F_{6 ; 2}^{(\mathrm{WL})}-F_{6 ; 2}^{(\mathrm{BDS})}$ should also be invariant.

As an example, let us consider six light-like four-dimensional vectors $p_{i}^{\mu}$,

$$
\begin{array}{lll}
p_{1}=(1,1,0,0), & p_{2}=(-1, p, p, 0), & p_{3}=(1,-p, p, 0), \\
p_{4}=(-1,-1,0,0), & p_{5}=(1,-p,-p, 0), & p_{6}=(-1, p,-p, 0),
\end{array}
$$

with $p=1 / \sqrt{2}$ and $\sum_{i} p_{i}^{\mu}=0$. These vectors define the external momenta of the gluons in the six-gluon amplitude. Applying the duality relation $p_{i}=x_{i+1}-x_{i}$, we evaluate the corresponding distances (273),

$$
\begin{aligned}
K^{(\mathrm{a})}: & x_{14}^{2}=x_{15}^{2}=x_{24}^{2}=x_{25}^{2}=-2, \quad x_{36}^{2}=-2-2 \sqrt{2}, \\
x_{13}^{2} & =x_{35}^{2}=x_{26}^{2}=x_{46}^{2}=-2-\sqrt{2},
\end{aligned}
$$

and the conformal cross-ratios (270),

$$
u_{1}=u_{3}=\frac{1}{2}+\frac{1}{2} \sqrt{2}, \quad u_{2}=1 .
$$

By construction, this kinematical configuration satisfies the Gram determinant constraint (274). To define the conformal transformations (276), we choose an arbitrary reference four-vector $x_{1}^{\mu}=\left(x_{1}^{0}, x_{1}^{1}, x_{1}^{2}, x_{1}^{3}\right)$ and reconstruct the remaining $x$-vectors according to $x_{i+1}^{\mu}=x_{i}^{\mu}+p_{i}^{\mu}$ (due to translation invariance, the Wilson loop does not depend on the choice of $x_{1}^{\mu}$ ). Then, relation (275) implies that the function $R_{\mathrm{W}}$ evaluated for the kinematical configuration (276) should be the same as for the original configuration $K$.

As an example, we choose $x_{1}^{\mu}=(1,1,1,1)$ and apply the conformal transformation (276) to $K^{(a)}$ defined in (278) to obtain the new kinematical configuration

$$
\begin{aligned}
K^{(\mathrm{b})}: & x_{14}^{2}=x_{15}^{2}=x_{24}^{2}=x_{25}^{2}=-\frac{1}{2}-\frac{1}{4} \sqrt{2}, \quad x_{36}^{2}=-1-\frac{3}{4} \sqrt{2}, \\
& x_{13}^{2}=-\frac{3}{2}-\sqrt{2}, \quad x_{35}^{2}=-\frac{5}{2}-\frac{7}{4} \sqrt{2}, \quad x_{26}^{2}=-\frac{1}{4}-\frac{1}{8} \sqrt{2}, \\
& x_{46}^{2}=-\frac{3}{8}-\frac{1}{4} \sqrt{2} .
\end{aligned}
$$

The results of our numerical tests are summarised in Table 1, They clearly show that $F_{6 ; 2}^{(\mathrm{WL})}$ and $F_{6 ; 2}^{(\mathrm{BDS})}$ vary under conformal transformations, whereas their difference $R_{\mathrm{W}}=F_{6 ; 2}^{(\mathrm{WL})}-F_{6 ; 2}^{(\mathrm{BDS})}$ stays invariant.

We recall that in four dimensions the kinematical invariants (273) have to verify the Gram determinant constraint (274). This relation $G[K]=0$ is invariant under the conformal transformations (276), simply because the conformal boosts map six light-like vectors $p_{i}^{\mu}$ into another set of light-like vectors. There exist, however, certain kinematical configurations $K^{\prime}$ for which $u_{i}[K]=u_{i}\left[K^{\prime}\right]$ but $G\left[K^{\prime}\right] \neq 0$. Since the difference function $R_{\mathrm{W}}$ only depends on the $u$-variables, its value should be insensitive to the Gram determinant condition 44 . For example, consider the following kinematical configuration

$$
\begin{aligned}
K^{(\mathrm{c})}: & x_{14}^{2}=x_{15}^{2}=-1, \quad x_{24}^{2}=x_{25}^{2}=-2, \quad x_{36}^{2}=-2-2 \sqrt{2}, \\
& x_{13}^{2}=-1-1 / \sqrt{2}, \quad x_{35}^{2}=x_{26}^{2}=x_{46}^{2}=-2-\sqrt{2} .
\end{aligned}
$$

The corresponding conformal cross-ratios (270) are given by (279), but $G\left[K^{(\mathrm{c})}\right] \neq 0$. We verified numerically that $R_{\mathrm{W}}\left[K^{(\mathrm{a})}\right]=R_{\mathrm{W}}\left[K^{(\mathrm{b})}\right]=R_{\mathrm{W}}\left[K^{(\mathrm{c})}\right]$ with accuracy $<10^{-5}$ (see Table 1). This observation allows us to study the function $R_{\mathrm{W}}\left(u_{1}, u_{2}, u_{3}\right)$ without any reference to the Gram determinant condition.

\footnotetext{
${ }^{44}$ We recall that the functions entering $\left[(275)\right.$ can be defined for configurations $K^{\prime}$ satisfying $G\left[K^{\prime}\right] \neq 0$.
} 


\begin{tabular}{||c||c|c|c||}
\hline \hline Kinematical point & $F_{6 ; 2}^{(\mathrm{WL})}$ & $F_{6 ; 2}^{(\mathrm{BDS})}$ & $R_{\mathrm{W}}$ \\
\hline \hline$K^{(\mathrm{a})}$ & -5.014825 & -14.294864 & 9.280039 \\
\hline$K^{(\mathrm{b})}$ & -6.414907 & -15.694947 & 9.280040 \\
\hline$K^{(\mathrm{c})}$ & -5.714868 & -14.994906 & 9.280038 \\
\hline
\end{tabular}

Table 1: Two-loop contributions to the hexagon Wilson loop, $F_{6 ; 2}^{(\mathrm{WL})}$, to the BDS ansatz, $F_{6 ; 2}^{(\mathrm{BDS})}$, and to their difference, $R_{\mathrm{W}}$, evaluated for three kinematical configurations (278), (280) and (281) corresponding to the same values of $u_{1}, u_{2}$ and $u_{3}$, Eq. (279).

\subsection{Collinear behaviour}

We recall that for the six-gluon amplitude $\mathcal{M}_{6}$ depending on light-like momenta, $\sum_{i=1}^{6} p_{i}^{\mu}=0$ and $p_{i}^{2}=0$, the collinear limit amounts to letting, e.g. $p_{5}^{\mu}$ and $p_{6}^{\mu}$ be nearly collinear (see e.g. 552 for more details), so that $\left(p_{5}+p_{6}\right)^{2} \rightarrow 0$ and

$$
p_{5}^{\mu} \rightarrow z P^{\mu}, \quad p_{6}^{\mu} \rightarrow(1-z) P^{\mu},
$$

with $P^{2}=0$ and $0<z<1$ being the momentum fraction. Using the identification $p_{i}^{\mu}=$ $x_{i+1}^{\mu}-x_{i}^{\mu}$, we translate these relations into properties of the corresponding Wilson loop $W\left(C_{6}\right)$. We find that the cusp at point 6 is 'flattened' in the collinear limit and the contour $C_{6}$ reduces to one with five cusps. In terms of the distances $x_{i j}^{2}$, the collinear limit amounts to

$$
\begin{aligned}
x_{15}^{2} & \rightarrow 0, & x_{36}^{2} & \rightarrow z x_{13}^{2}+(1-z) x_{35}^{2}, \\
x_{46}^{2} & \rightarrow z x_{14}^{2}, & x_{26}^{2} & \rightarrow(1-z) x_{25}^{2},
\end{aligned}
$$

while the other distances $x_{13}^{2}, x_{24}^{2}, x_{25}^{2}, x_{35}^{2}$ remain unchanged. For the conformal cross-ratios the relation (283) implies

$$
u_{1} \rightarrow u, \quad u_{2} \rightarrow 0, \quad u_{3} \rightarrow 1-u,
$$

with $u=z x_{13}^{2} /\left(z x_{13}^{2}+(1-z) x_{35}^{2}\right)$ fixed. As was already mentioned, the relation (268) is consistent with the collinear limit of the six-gluon amplitude provided that, in the limit (284), the function $R\left(u_{1}, u_{2}, u_{3}\right)$ approaches a finite value independent of the kinematical invariants. The same property can be expressed as follows (we recall that the function $R\left(u_{1}, u_{2}, u_{3}\right)$ is totally symmetric)

$$
R(0, u, 1-u)=c,
$$

with $c$ being a constant. Using our two-loop results for the finite part $F_{6}$, we performed thorough numerical tests of the relation (285) for different kinematical configurations of the contour $C_{6}$.

We found that, in agreement with (285), the limiting value of the function $R^{(2)}(\gamma, u, 1-u)$ as $\gamma \rightarrow 0$ does not depend on $u$. Since the duality relation (198) is not sensitive to the value of this constant, it is convenient to subtract it from $R^{(2)}(\gamma, u, 1-u)$ and introduce the function

$$
\widehat{R}^{(2)}(\gamma, u, 1-u)=c-R^{(2)}(\gamma, u, 1-u)
$$

which satisfies $\widehat{R}^{(2)}(0, u, 1-u)=0$. To summarise our findings, in Fig. 30 we plot the function $\widehat{R}^{(2)}(\gamma, u, 1-u)$ against $\gamma$ for different choices of the parameter $0<u<1$ and in Fig. 31 the same function against $u$ for different choices of the parameter $\gamma$. The important region for the collinear limit is where $\gamma$ is close to zero. We also give numerical values for a range of values of $\gamma$ 


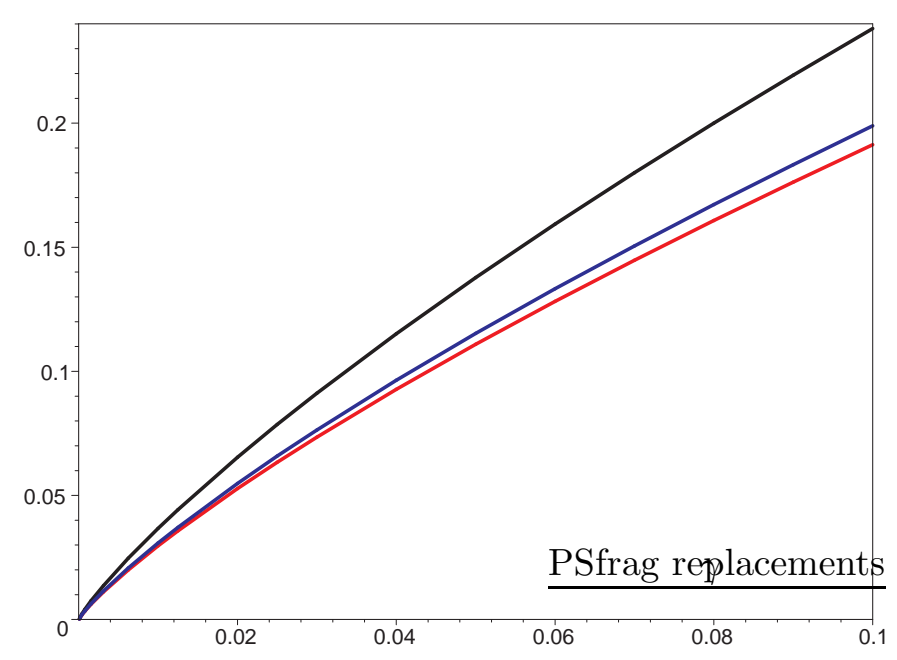

Figure 30: The $\gamma$-dependence of the function $\widehat{R}^{(2)}(\gamma, u, 1-u)$, Eq. (286), for different values of the parameter $u=0.5$ (lower curve), $u=0.3$ (middle curve) and $u=0.1$ (upper curve).

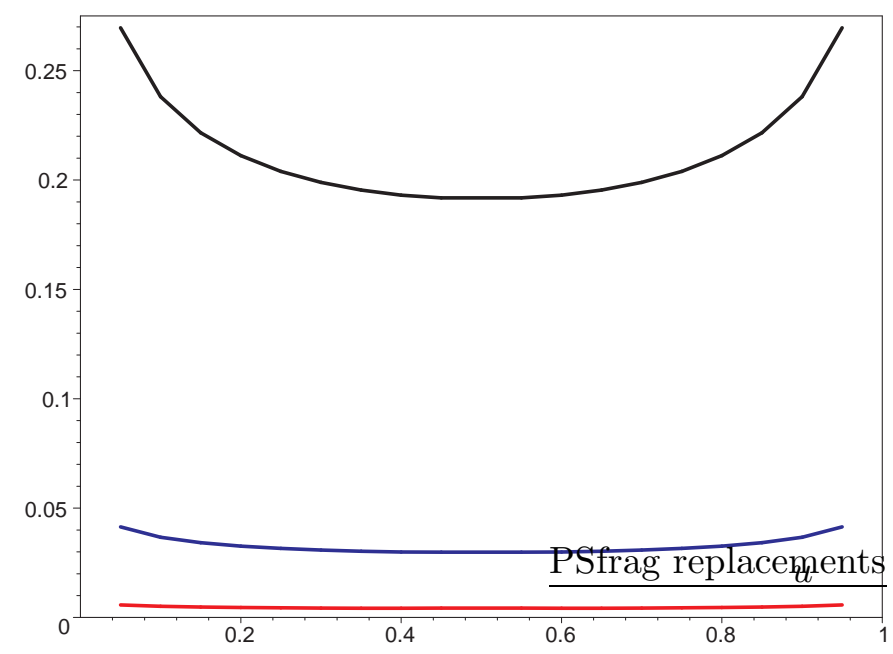

Figure 31: The $u$-dependence of the function $\widehat{R}^{(2)}(\gamma, u, 1-u)$, Eq. (286), for different values of the parameter $\gamma=0.001$ (lower curve), $\gamma=0.01$ (middle curve) and $\gamma=0.1$ (upper curve).

such that one can see how the function $R^{(2)}\left(u_{1}, u_{2}, u_{3}\right)$ varies in the particular parametrisation $u_{1}=\gamma, u_{2}=u, u_{3}=1-u$.

So, in conclusion, our explicit $n=6$ Wilson loop calculation [90] has shown that at two loops there exists a non-trivial 'remainder' function $R_{6}$ satisfying the conformal Ward identities and the collinear limit condition. The crucial test then was to compare the results of our Wilson loop calculation with a parallel two-loop six-gluon amplitude calculation, in order to check whether the proposed duality between Wilson loops and gluon amplitudes continues to hold at this level (which, if true, automatically implies the breakdown of the BDS ansatz). The results of the six-gluon calculation have become available very recently [92, and we present the comparison in section 9.4 . 


\subsection{The hexagon Wilson loop versus the six-gluon MHV amplitude}

The recently completed two-loop six-gluon amplitude calculation 92 found that indeed the BDS ansatz fails at that level, i.e.

$$
R_{\mathrm{A}}=F_{6 ; 2}^{(\mathrm{MHV})}-F_{6 ; 2}^{(\mathrm{BDS})} .
$$

with a nontrivial 'remainder function'. We now compare numerically the 'remainder' function $R_{\mathrm{A}}$ with the analogous quantity defined for the hexagonal Wilson loop in (275). In order to test the duality relation (195), we have to show that for general kinematical configurations $K$ in (273), we have

$$
R_{\mathrm{W}}[K]-R_{\mathrm{A}}[K]=c_{\mathrm{W}} .
$$

Since $R_{\mathrm{W}}$ is a function of $u_{1}, u_{2}, u_{3}$ only, this relation would imply that so is $R_{\mathrm{A}}$.

To get rid of the constant $c_{\mathrm{W}}$, which has lower numerical precision than the evaluations for generic kinematics, we subtract from (288) the same relation evaluated for some reference kinematical configuration $K^{(0)}$,

$$
R_{\mathrm{A}}[K]-R_{\mathrm{A}}\left[K^{(0)}\right]=R_{\mathrm{W}}[K]-R_{\mathrm{W}}\left[K^{(0)}\right] .
$$

The numerical tests of this relation for different kinematical configurations are summarised in Table 2 ,

\begin{tabular}{||c|c||c|c||}
\hline \hline Kinematical point & $\left(u_{1}, u_{2}, u_{3}\right)$ & $R_{\mathrm{W}}-R_{\mathrm{W}}^{(0)}$ & $R_{\mathrm{A}}-R_{\mathrm{A}}^{(0)}$ \\
\hline \hline$K^{(1)}$ & $(1 / 4,1 / 4,1 / 4)$ & $<10^{-5}$ & $-0.018 \pm 0.023$ \\
\hline$K^{(2)}$ & $(0.547253,0.203822,0.88127)$ & -2.75533 & $-2.753 \pm 0.015$ \\
\hline$K^{(3)}$ & $(28 / 17,16 / 5,112 / 85)$ & -4.74460 & $-4.7445 \pm 0.0075$ \\
\hline$K^{(4)}$ & $(1 / 9,1 / 9,1 / 9)$ & 4.09138 & $4.12 \pm 0.10$ \\
\hline$K^{(5)}$ & $(4 / 81,4 / 81,4 / 81)$ & 9.72553 & $10.00 \pm 0.50$ \\
\hline
\end{tabular}

Table 2: Comparison of the deviation from the BDS ansatz of the Wilson loop, $R_{\mathrm{W}}$, and of the six-gluon amplitude, $R_{\mathrm{A}}$, evaluated for the kinematical configurations (290). Here, $R_{\mathrm{W}}^{(0)}=$ $R_{\mathrm{W}}(1 / 4,1 / 4,1 / 4)=13.26530$ and $R_{\mathrm{A}}^{(0)}=R_{\mathrm{A}}(1 / 4,1 / 4,1 / 4)=1.0937 \pm 0.0057$ denote the same quantities evaluated at the reference kinematical point $K^{(0)}$. The numerical results for $R_{\mathrm{A}}$ and $R_{\mathrm{A}}^{(0)}$ are taken from Ref. [92]. 


$$
\begin{aligned}
K^{(0)}: & x_{i, i+2}^{2}=-1, \quad x_{i, i+3}^{2}=-2 ; \\
K^{(1)}: & x_{13}^{2}=-0.7236200, \quad x_{24}^{2}=-0.9213500, \quad x_{35}^{2}=-0.2723200, \quad x_{46}^{2}=-0.3582300, \\
& x_{15}^{2}=-0.4235500, \quad x_{26}^{2}=-0.3218573, \quad x_{14}^{2}=-2.1486192, \quad x_{25}^{2}=-0.7264904, \\
& x_{36}^{2}=-0.4825841 ; \\
K^{(2)}: & x_{13}^{2}=-0.3223100, \quad x_{24}^{2}=-0.2323220, \quad x_{35}^{2}=-0.5238300, \quad x_{46}^{2}=-0.8237640, \\
& x_{15}^{2}=-0.5323200, \quad x_{26}^{2}=-0.9237600, \quad x_{14}^{2}=-0.7322000, \quad x_{25}^{2}=-0.8286700 \\
& x_{36}^{2}=-0.6626116 ; \quad x_{14}^{2}=-1 / 2, \quad x_{25}^{2}=-5 / 8, \quad x_{36}^{2}=-17 / 14 ; \\
K^{(3)}: & x_{i, i+2}^{2}=-1, \quad x_{i, i+3}^{2}=-3 ; \\
K^{(4)}: & x_{i, i+2}^{2}=-1, \quad x_{i, i+3}^{2}=-9 / 2 .
\end{aligned}
$$

Among the six configurations in equation (290) only the first four verify the Gram determinant condition 274. Also, the configurations $K^{(0)}$ and $K^{(1)}$ are related to each other by a conformal transformation. This explains why the first entry in the third column of Table 2 is almost zero, and also reflects the high precision of the numerical evaluation for the Wilson loop. Comparing the numerical values for the six-gluon amplitude and the hexagon Wilson loop, we observe that their finite parts coincide within the error bars. Therefore, we conclude that the duality relation (195) is satisfied to two loops at least. We consider this very strong evidence that the duality should hold to all orders in the coupling, although the 'remainder' function $R_{n}$ is likely to receive corrections at each loop order.

\section{Conclusions and outlook}

In this report, we studied a new duality between Wilson loops and gluon amplitudes. The latter was motivated mainly by work of Alday and Maldacena, which showed that there is a relation between gluon amplitudes and Wilson loops at strong coupling, using the AdS/CFT correspondence. Unexpectedly, the duality seems to hold also at weak coupling. Previous studies of the duality were restricted to one loop. In this thesis we computed the expectation value of the rectangular and pentagonal Wilson loops at two loops. A comparison with the corresponding MHV gluon amplitudes shows that the duality holds.

As was emphasised in this report, broken (dual) conformal symmetry plays an important role on both sides of the duality. We showed that the integrals contributing to the loop corrections of the four-gluon amplitude up to four loops are all 'pseudo-conformal' in the sense explained in section 3.2. The origin of the dual conformal symmetry for gluon amplitudes remains puzzling. For the Wilson loops, however, it is completely understood and controlled by an all-orders Ward identity, which we derived in this thesis.

The case $n=6$ provides a test of the duality relation beyond (dual) conformal symmetry. What makes the hexagonal Wilson loops and the six-gluon MHV amplitude agree? It seems natural to conjecture that (dual) conformal symmetry is part of a larger symmetry group, which is yet to be discovered. Such a speculation is further supported by the fact that both Wilson loops and gluon amplitudes are intimately related to the cusp anomalous relation, which in turn is governed by integrability. 
The results obtained give strong support for the duality between Wilson loops and gluon scattering amplitudes in $\mathcal{N}=4 \mathrm{SYM}$. In our opinion, given the evidence presented here, it is likely that the duality is true to all orders in the coupling constant, and for an arbitrary number of points/gluons. This duality gives rise to several intriguing possibilities.

The calculation of the pole part of the $n$-cusp Wilson loop in [87] was carried out analytically for arbitrary $n$ at two loops, in agreement with the general formula (176). As explained in [87, one can define 'auxiliary' diagrams to subtract the pole terms, thereby providing a definition of the finite part of the two-loop $n$-cusp Wilson loop in terms of a set of finite parametric integrals. We studied the latter numerically in the $n=5,6$ case, as discussed in this report, and a generalisation to $n>6$ should not be difficult.

It would be very interesting if the arguments of Alday and Maldacena that related gluon scattering amplitudes and Wilson loops at strong coupling could be extended to arbitrary values of the coupling constant. This would constitute a proof of the duality via the AdS/CFT correspondence, or conversely, such a derivation would be an impressive test of the AdS/CFT correspondence.

So far, the duality is limited to maximally helicity violating (MHV) amplitudes. It is natural to wonder whether or how the duality extends to amplitudes with different helicity configurations, as for example NMHV. An obvious question is then how to match the helicity structure of the NMHV amplitudes to the Wilson loops, which are helicity independent. One may speculate that there could exist suitable modifications of the Wilson loops such that they match those amplitudes. For example, one could consider Wilson loops with local operator insertions.

A related question is what the dual conformal properties of NMHV amplitudes are. This will be the subject of a forthcoming paper together with J. Drummond, G. Korchemsky and E. Soktachev.

Another interesting possibility, in our opinion, would be to consider a different point of the moduli space of $\mathcal{N}=4 \mathrm{SYM}$, such that some particles become massive. One can ask the question whether scattering amplitudes of massive on-shell gluons are still dual to the corresponding Wilson loops, whose sides are no longer light-like (because of the mass). On the other hand, for finite masses the conformal symmetry that was important in our analysis would be lost for such a configuration.

\section{Acknowledgements}

First of all, it is a pleasure to thank my advisor, Emery Sokatchev, for initiating me to this interesting area of research and for sharing his knowledge. Special thanks are also due to Andrei Belitsky, James Drummond and Gregory Korchemsky for numerous discussions and encouragement. I have also profitted from discussions with Costas Bachas, Benjamin Basso, Niklas Beisert, Massimo Bianchi, Fawzi Boudjema, Lance Dixon, Vladimir Dobrev, Burkhard Eden, Johanna Erdmenger, Giovanni Feverati, Laurent Gallot, François Gieres, Jean-Phillipe Guillet, Paul Heslop, Candide Jarczak, Dieter Müller, Eric Pilon, Jan Plefka, Eric Ragoucy, Paul Sorba, Yassen Stanev, Kelly Stelle, Raymond Stora, Frank Thuiller, Ivan Todorov and Arkady Tseytlin. I am grateful to Nans Baro, Livia Ferro, Charlotte Grosse-Wiesmann and Konstantin Wiegandt for pointing out typos in the draft to me.

I would like to thank the Laboratoire d'Annecy-le-Vieux de Physique Théorique (LAPTH) and its director, Patrick Aurenche, for providing a stimulating working environment during the 
last three years. The secretaries, Nicole Berger, Véronique Jonnery, Dominique Turc and Virginie Malaval have helped considerably to simplify the administrative part of this thesis. It has been great to share the experience of doing a $\mathrm{PhD}$ with the fellow students Nans Baro, Samuel Belliard, Christophe Bernicot, Candide Jarczak, Ninh Le Duc, Grégory Sanguinetti, Wessel Valkenburg. They and the other members of LAPTH made the three years much more enjoyable and worthwile.

This research was supported in part by the French Agence Nationale de la Recherche under grant ANR-06-BLAN-0142. Partial financial support from the Université de Savoie through an Explora'Doc grant and travel money through an APS grant (2005) is gratefully acknowledged. It is a pleasure to acknowledge the warm hospitality extended to me by the Theory Group of the Dipartimento di Fisica, Università di Roma "Tor Vergata", where part of this work was done (from April 2007 to September 2007). Special thanks are due to Yassen Stanev for his hospitality and numerous enlightening discussions.

I would like to thank the theoretical physics sections of the Università di Roma "Tor Vergata", the Università Torino, the Univerisità Milano Bicocca, the ETH Zürich, and Imperial College London, where part of this work was presented, for their seminar invitations. In particular I would like to thank the participants of these seminars for their comments and for discussions, which certainly improved the way the material is presented in this thesis.

Finally, this thesis would not have been written had it not been for the university education I received. At the Universität Augsburg I would like to thank in particular Reinhard Schertz and Gert-Ludwig Ingold for their excellent lectures that aroused my interest in mathematics and theoretical physics, and similarly Daniel Décamp at the Univerité de Savoie for initiating me to the world of elementary particle physics. Finally, I would like to thank Luc Frappat, Frank Thuiller and Jean-Claude Le Guillou for their help in organising my Erasmus year at the Université de Savoie, and Frainçois Delduc for the organisation of the theoretical physics master's programme at the Ecole Normale Supérieure de Lyon.

\section{A Alternative proof of $\Phi^{(3)}=\Psi^{(3)}$ using the Mellin-Barnes rep- resentation}

In this section we show how the above identity between the off shell triple box and tennis court integrals derived in section 2.6 can also be obtained by means of the method of Mellin-Barnes (MB) representation.

\section{A.1 Introduction to the Mellin-Barnes technique}

This method is one of the most powerful methods of evaluating individual Feynman integrals. It is especially successful for evaluating four-point Feynman integrals. For massless off-shell four-point integrals, first results were obtained by means of MB representation in [143, 144]. In the context of dimensional regularisation, with the space-time dimension $d=4-2 \epsilon$ as a regularisation parameter, two alternative strategies for resolving the structure of singularities in $\epsilon$ were suggested in [203, 204 where first results on evaluating four-point on-shell massless Feynman integrals were obtained. Then these strategies were successfully applied to evaluate massless on-shell double [203, 204, 205, 206, 207, 208, 209] and triple 210, 211, 39] boxes, with results written in terms of harmonic polylogarithms 212, double boxes with one leg off shell [213, 214] and massive on-shell double boxes [215, 216, 217, 218] (see also chapter 5 of [219]). It 


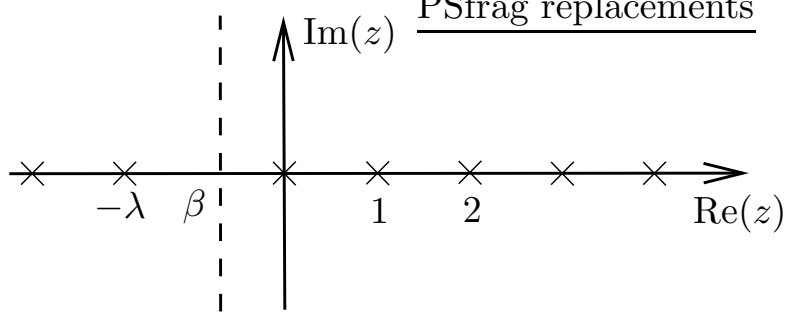

Figure 32: Position of the integration contour (dashed line) and poles of the integrand (crosses) in formula (291), for real $\lambda$.

is based on the MB representation

$$
\frac{1}{(X+Y)^{\lambda}}=\frac{1}{\Gamma(\lambda)} \frac{1}{2 \pi i} \int_{\beta-i \infty}^{\beta+i \infty} \frac{Y^{z}}{X^{\lambda+z}} \Gamma(\lambda+z) \Gamma(-z) \mathrm{d} z
$$

applied to replace a sum of terms raised to some power by their product to some powers. The integration in (291) goes along a straight line parallel to the imaginary axis, with $-\operatorname{Re}(\lambda)<$ $\beta<0$, so that the poles of $\Gamma(\lambda+z)$ lie on its left and those of $\Gamma(-z)$ on its right, as shown in Fig. 32. Formula (291) is easy to understand. If $Y<X$, we can close the integration contour on the right. By the residue theorem, we will pick up contributions from the poles of $\Gamma(-z)$, leading to

$$
\frac{1}{\Gamma(\lambda)} \frac{1}{2 \pi i} \int_{\beta-i \infty}^{\beta+i \infty} \frac{Y^{z}}{X^{\lambda+z}} \Gamma(\lambda+z) \Gamma(-z) \mathrm{d} z=X^{-\lambda} \sum_{n=0}^{\infty}(-1)^{n} \frac{\Gamma(\lambda+n)}{\Gamma(\lambda)}\left(\frac{Y}{X}\right)^{n} .
$$

The right-hand side of (292) is indeed the series expansion of $(X+Y)^{-\lambda}$ for $Y<X$. However equation (291) is also valid for $X<Y$, which can be seen by closing the integration contour on the left.

The first step of the method is the derivation of an appropriate MB representation. It is very desirable to do this for general powers of the propagators (indices) and irreducible numerators. On the one hand, this provides crucial checks of a given MB representation using simple partial cases. (For example, one can shrink either horizontal or vertical lines to points, i.e. set the corresponding indices to zero, and obtain simple diagrams quite often expressed in terms of gamma functions.) On the other hand, such a general derivation provides unambiguous prescriptions for choosing integration contours (see details in [219]).

\section{A.2 Example: One-loop integral}

As an example, let us come back to the one-loop integral $\Phi^{(1)}$. Using the relation to the threepoint integral (73) it is straightforward to obtain the following parameter integral representation:

$$
\Phi^{(1)}(u, v)=(2 \pi i)^{-2} \int_{0}^{1} \frac{d \beta_{1} d \beta_{2} d \beta_{3} \delta\left(1-\beta_{1}-\beta_{2}-\beta_{3}\right)}{\beta_{1} \beta_{2} u+\beta_{2} \beta_{3} v+\beta_{1} \beta_{3}},
$$

or, after a change of variables,

$$
\Phi^{(1)}(u, v)=(2 \pi i)^{-2} \int_{0}^{1} d x \int_{0}^{1} d \lambda \frac{\lambda}{\lambda(x u+\bar{x} v)+\bar{\lambda} x \bar{x}},
$$

where $\bar{x}=1-x$ and $\bar{\lambda}=1-\lambda$. Then, one uses the Mellin Barnes formula (291) twice in order to factorise the denominator in (293), i.e.

$$
\frac{1}{a+b+c}=\int_{-i \infty}^{i \infty} d z_{1} \int_{-i \infty}^{i \infty} d z_{2} \Gamma\left(-z_{1}\right) \Gamma\left(-z_{2}\right) \Gamma\left(1+z_{1}+z_{2}\right) a^{z_{1}} b^{z_{2}} c^{\left(-1-z_{1}-z_{2}\right)}
$$


As usual, the integration contours are straight vertical lines, and they are chosen between the poles of the $\Gamma$ functions, i.e.

$$
\operatorname{Re}\left(z_{1}\right)<0, \quad \operatorname{Re}\left(z_{2}\right)<0, \quad \operatorname{Re}\left(z_{1}\right)+\operatorname{Re}\left(z_{2}\right)>-1
$$

so one could choose $\operatorname{Re}\left(z_{1}\right)=-0.2$ and $\operatorname{Re}\left(z_{2}\right)=-0.4$ for example. The integration over the parametric integrals is now straightforward, and we obtain the MB representation [143] (see also [219])

$$
\Phi^{(1)}(u, v)=(2 \pi i)^{-2} \int_{-i \infty}^{i \infty} d z_{1} \int_{-i \infty}^{i \infty} d z_{2} u^{z_{1}} v^{z_{2}} \Gamma^{2}\left(-z_{1}\right) \Gamma^{2}\left(-z_{2}\right) \Gamma^{2}\left(1+z_{1}+z_{2}\right) .
$$

For $0<u, v<1$ one can close the integration contours in (297) on the right and use the residue theorem to compute the integrals. This yields the result as a series expansion in $u$ and $v$.

The representation (297) is also useful for studying the limiting behaviour of $\Phi^{(1)}$. As an example, consider the OPE limit $x_{12} \rightarrow$ 0, i.e. $u \rightarrow 0$, cf. (77). Since $\operatorname{Re}\left(z_{1}\right)<0$ the limit cannot be taken naively in the integrand of (297). Therefore, we deform the contour such that $0<\operatorname{Re}\left(z_{1}\right)<1$. In doing so, we cross a pole at $u=0$ and hence pick up a contribution from the residue of $u^{z_{1}} \Gamma^{2}\left(-z_{1}\right) \Gamma^{2}\left(1+z_{1}+z_{2}\right)$. Neglecting terms that vanish as $u \rightarrow 0$, we obtain

$$
\begin{aligned}
\Phi^{(1)}(u, v)= & -(2 \pi i)^{-1} \ln (u) \int_{-i \infty}^{i \infty} v^{z_{2}} \Gamma^{2}\left(-z_{2}\right) \Gamma^{2}\left(1+z_{2}\right) d z_{2} \\
& -(2 \pi i)^{-1} \int_{-i \infty}^{i \infty} v^{z_{2}} \Gamma^{2}\left(-z_{2}\right) \Gamma^{2}\left(1+z_{2}\right)\left[\Psi\left(1+z_{2}\right)+\gamma_{E}\right] d z_{2}+O(u) .
\end{aligned}
$$

In the second line $\gamma_{E}$ is the Euler constant and for integer $v$ the expression $\Psi(1+v)+\gamma_{E}$ is equal to an harmonic sum $S_{v}=\sum_{n=1}^{v} 1 / n$. Computing the integrals in (298) by closing the contour on the right we pick up residues from $v=0,1, \ldots$

$$
\begin{aligned}
\operatorname{Res}_{z_{2}=n}\left[v^{z_{2}} \Gamma^{2}\left(-z_{2}\right) \Gamma^{2}\left(1+z_{2}\right)\right] & =v^{n} \ln v \\
\operatorname{Res}_{z_{2}=n}\left[v^{z_{2}} \Gamma^{2}\left(-z_{2}\right) \Gamma^{2}\left(1+z_{2}\right)\left(\Psi\left(1+z_{2}\right)+\gamma_{E}\right)\right] & =2 v^{n}\left[\Psi^{\prime}(1+n)+S_{n} \ln v\right] .
\end{aligned}
$$

The resulting series can be summed. The first is just a geometrical series, and the second one can be done as well (e.g. using Mathematica). We obtain

$$
\Phi^{(1)}(u, v)=\frac{1}{1-v}\left[\ln (u) \ln (v)-2 \ln (v) \ln (1-u)+\frac{\pi^{2}}{3}-2 \mathrm{Li}_{2}(v)\right]+O(u),
$$

or equivalently (still for $0<v<1$ ),

$$
\Phi^{(1)}(u, v)=\frac{1}{1-v}\left[\ln (u) \ln (v)+2 \operatorname{Li}_{2}(1-v)\right]+O(u),
$$

in agreement with (77). Although this expression was obtained for $0<v<1$, it can be analytically continued to all $v>0$.

Many other examples of the application of the Mellin-Barnes technique to the evaluation of Feynman integrals can be found in the book [219].

\section{A.3 Alternative proof of the magic identity at three loops}

Let us now use the MB technique to find an alternative proof of the 'turning identity' $\Phi^{(3)}=\Psi^{(3)}$. We consider the off shell triple box and tennis court labelled as shown in Figs. 33 and 34, with 


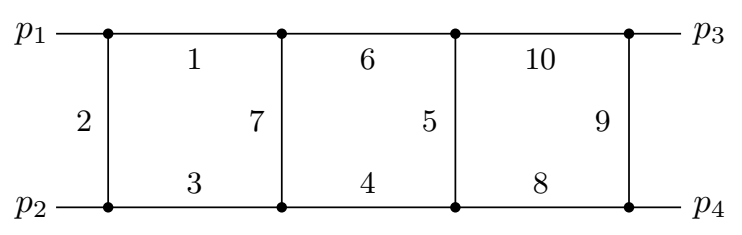

Figure 33: Labelled triple box.

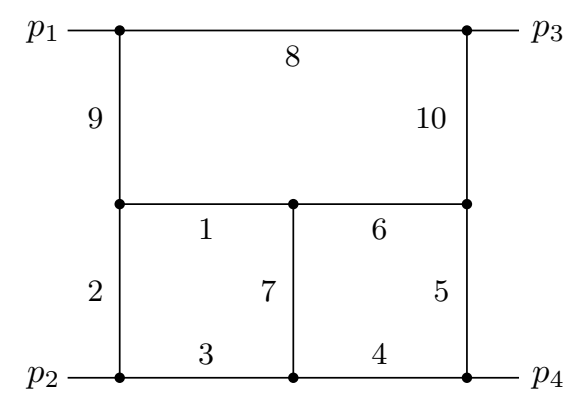

Figure 34: Labelled tennis court.

general powers of the propagators and one irreducible numerator in the tennis court chosen as $\left[\left(l_{1}+l_{3}\right)^{2}\right]^{-a_{11}}$, where $l_{1,3}$ are the momenta flowing through lines 1 and 3 in the same direction.

Experience shows that a minimal number of MB integrations for planar diagrams is achieved if one introduces MB integrations loop by loop, i.e. one derives a MB representation for a oneloop subintegral, inserts it into a higher two-loop integral, etc. This straightforward strategy provides the following 15-fold MB representations for the dimensionally regularised off-shell triple box and tennis court with general indices:

$$
\begin{aligned}
T_{1} & \left(a_{1}, \ldots, a_{10} ; s, t, p_{1}^{2}, p_{2}^{2}, p_{3}^{2}, p_{4}^{2} ; \epsilon\right)=\frac{\left(i \pi^{d / 2}\right)^{3}(-1)^{a}(-s)^{6-a-3 \epsilon}}{\prod_{j=2,4,5,6,7,9} \Gamma\left(a_{j}\right) \Gamma\left(4-2 \epsilon-a_{4,5,6,7}\right)} \\
& \times \frac{1}{(2 \pi i)^{15}} \int_{-i \infty}^{+i \infty} \prod_{j=1}^{15} \mathrm{~d} z_{j} \frac{\left(-p_{1}^{2}\right)^{z_{12}}\left(-p_{2}^{2}\right)^{z_{13}}\left(-p_{3}^{2}\right)^{z_{4,9,14}}\left(-p_{4}^{2}\right)^{z_{5,10,15}}(-t)^{z_{11}}}{(-s)^{z_{4,5,9,10,11,12,13,14,15}}} \\
& \times \frac{\Gamma\left(a_{9}+z_{11,12,13}\right) \Gamma\left(a_{7}+z_{1,2,3}\right) \Gamma\left(2-\epsilon-a_{5,6,7}-z_{1,2,4}\right) \Gamma\left(2-\epsilon-a_{4,5,7}-z_{1,3,5}\right)}{\Gamma\left(a_{1}-z_{2}\right) \Gamma\left(a_{3}-z_{3}\right) \Gamma\left(4-2 \epsilon-a_{1,2,3}+z_{1,2,3}\right)} \\
& \times \frac{\Gamma\left(a_{5}+z_{1,4,5}\right) \Gamma\left(a_{4,5,6,7}+\epsilon-2+z_{1,2,3,4,5}\right) \Gamma\left(z_{11,14,15}-z_{6}\right)}{\Gamma\left(a_{8}-z_{7}\right) \Gamma\left(a_{10}-z_{8}\right) \Gamma\left(4-2 \epsilon-a_{8,9,10}+z_{6,7,8}\right)} \\
& \times \Gamma\left(2-\epsilon-a_{8,9}+z_{6,7}-z_{11,12,14}\right) \Gamma\left(2-a_{2,3}-\epsilon+z_{1,3}-z_{6,8,10}\right) \\
& \times \Gamma\left(a_{8,9,10}+\epsilon-2+z_{11,12,13,14,15}-z_{6,7,8}\right) \Gamma\left(2-\epsilon-a_{9,10}+z_{6,8}-z_{11,13,15}\right) \\
& \times \Gamma\left(a_{2}+z_{6,7,8}\right) \Gamma\left(2-\epsilon-a_{1,2}+z_{1,2}-z_{6,7,9}\right) \\
& \times \Gamma\left(z_{6,9,10}-z_{1}\right) \Gamma\left(a_{1,2,3}+\epsilon-2-z_{1,2,3}+z_{6,7,8,9,10}\right) \prod_{j=2,3,4,5,7, \ldots, 15} \Gamma\left(-z_{j}\right) ;
\end{aligned}
$$




$$
\begin{aligned}
& T_{2}\left(a_{1}, \ldots, a_{11} ; s, t, p_{1}^{2}, p_{2}^{2}, p_{3}^{2}, p_{4}^{2} ; \epsilon\right)=\frac{\left(i \pi^{d / 2}\right)^{3}(-1)^{a}(-s)^{6-a-3 \epsilon}}{\prod_{j=2,4,5,6,7,9} \Gamma\left(a_{j}\right) \Gamma\left(4-2 \epsilon-a_{4,5,6,7}\right)} \\
& \times \frac{1}{(2 \pi i)^{15}} \int_{-i \infty}^{+i \infty} \prod_{j=1}^{15} \mathrm{~d} z_{j} \frac{\left(-p_{1}^{2}\right)^{z_{12}}\left(-p_{2}^{2}\right)^{z_{13}}\left(-p_{3}^{2}\right)^{z_{5,10,14}}\left(-p_{4}^{2}\right)^{z_{15}+z_{8}}(-t)^{z_{11}}}{(-s)^{z_{5,8,10,11,12,13,14,15}}} \prod_{j=2}^{15} \Gamma\left(-z_{j}\right) \\
& \times \frac{\Gamma\left(a_{9}+z_{11,12,13}\right) \Gamma\left(a_{7}+z_{1,2,3}\right) \Gamma\left(2-a_{5,6,7}-\epsilon-z_{1,2,4}\right) \Gamma\left(2-a_{4,5,7}-\epsilon-z_{1,3,5}\right)}{\Gamma\left(a_{1}-z_{2}\right) \Gamma\left(a_{3}-z_{3}\right) \Gamma\left(4-2 \epsilon-a_{1,2,3}+z_{1,2,3}\right) \Gamma\left(a_{10}-z_{7}\right)} \\
& \times \frac{\Gamma\left(a_{5}+z_{1,4,5}\right) \Gamma\left(a_{4,5,6,7}+\epsilon-2+z_{1,2,3,4,5}\right) \Gamma\left(2-a_{2,3}-\epsilon+z_{1,3}-z_{6,8,10}\right)}{\Gamma\left(8-4 \epsilon-a-z_{5,6,8,10}\right) \Gamma\left(a_{8}-z_{4,9}\right) \Gamma\left(a_{1,2,3,4,5,6,7,11}+2 \epsilon-4+z_{4,5,6,7,8,9,10}\right)} \\
& \times \Gamma\left(6-a+a_{10}-3 \epsilon-z_{5,6,7,8,10,11,12,14}\right) \Gamma\left(a+3 \epsilon-6+z_{5,6,8,10,11,12,13,14,15}\right) \\
& \times \Gamma\left(a_{2}+z_{6,7,8}\right) \Gamma\left(2-\epsilon-a_{1,2}+z_{1,2}-z_{6,7,9}\right) \Gamma\left(6-3 \epsilon-a+a_{8}-z_{4,5,6,8,9,10,11,13,15}\right) \\
& \times \Gamma\left(z_{6,9,10}-z_{1}\right) \Gamma\left(a_{1,2,3}+\epsilon-2-z_{1,2,3}+z_{6,7,8,9,10}\right) \\
& \times \Gamma\left(a_{1,2,3,4,5,6,7,11}+2 \epsilon-4+z_{4,5,6,7,8,9,10,11,14,15}\right) .
\end{aligned}
$$

Here $a_{4,5,6,7}=a_{4}+a_{5}+a_{6}+a_{7}, a=\sum a_{i}, z_{11,12,13}=z_{11}+z_{12}+z_{13}$, etc. Moreover, the letters $s$ and $t$ denote the usual Mandelstam variables $s=\left(p_{1}+p_{2}\right)^{2}$ and $t=\left(p_{1}+p_{3}\right)^{2}$.

These representations are written for the Feynman integrals in Minkowski space. (This is rather convenient, in particular this allows one to put some of the legs on-shell.) The corresponding Euclidean versions are obtained by the replacements $-s \rightarrow s,-t \rightarrow t,-p_{1}^{2} \rightarrow p_{1}^{2}, \ldots$ and by omitting the prefactors $(-1)^{a}$ and $i^{3}$.

To calculate the triple box we need, i.e. $T_{1}^{(0)}=T_{1}(1, \ldots, 1)$ at $d=4$, we simply set all the indices $a_{i}$ to one. We cannot immediately set $\epsilon=0$ because there is $\Gamma(-2 \epsilon)$ in the denominator. The value of the integral is, of course, non-zero, so that some poles in $\epsilon$ arise due to the integration. To resolve the structure of poles one can apply Czakon's code [220], which provides the following value of the integral in the limit $\epsilon \rightarrow 0$ after relabelling the variables by $z_{10} \rightarrow$ $z_{2}, z_{14} \rightarrow z_{3}, z_{15} \rightarrow z_{4}, z_{11} \rightarrow z_{5}, z_{12} \rightarrow z_{6}$ :

$$
\begin{aligned}
T_{1}^{(0)} & =\frac{\left(i \pi^{2}\right)^{3}}{(2 \pi i)^{6}} \int_{-i \infty}^{+i \infty} \prod_{j=1}^{6} \mathrm{~d} z_{j} \frac{\left(-p_{1}^{2}\right)^{z_{6}}\left(-p_{2}^{2}\right)^{-1-z_{5,6}}\left(-p_{3}^{2}\right)^{-1-z_{5,6}\left(-p_{4}^{2}\right)^{z_{6}}(-t)^{z_{5}}}}{(-s)^{2-z_{5}}} \\
& \times \frac{\Gamma\left(1+z_{3,4}\right) \Gamma\left(1+z_{1}-z_{3,4,5}\right) \Gamma\left(z_{2,3,4,5}-z_{1}\right) \Gamma\left(z_{4}-z_{6}\right)}{\Gamma\left(1+z_{4}-z_{6}\right) \Gamma\left(1+z_{2,4}-z_{6}\right) \Gamma\left(2+z_{1,6}-z_{2,4}\right) \Gamma\left(2+z_{3,5,6}\right)} \prod_{j} \Gamma\left(-z_{j}\right) \\
& \times \Gamma\left(z_{2,4}-z_{6}\right)^{2} \Gamma\left(1+z_{1,6}-z_{2,4}\right)^{2} \Gamma\left(1+z_{5,6}\right) \Gamma\left(1+z_{3,5,6}\right) .
\end{aligned}
$$

To calculate the tennis court we need, i.e. $T_{2}^{(0)}=T_{2}(1, \ldots, 1,-1)$ at $d=4$, we proceed like in the previous case. Czakon's code provides the following integral (after relabelling $z_{10} \rightarrow z_{2}, z_{14} \rightarrow$ $\left.z_{3}, z_{15} \rightarrow z_{4}, z_{11} \rightarrow z_{5}, z_{12} \rightarrow z_{6}\right)$ :

$$
\begin{aligned}
T_{2}^{(0)}= & \frac{\left(i \pi^{2}\right)^{3}}{(2 \pi i)^{6}} \int_{-i \infty}^{+i \infty} \prod_{j=1}^{6} \mathrm{~d} z_{j} \frac{\left(-p_{1}^{2}\right)^{z_{6}}\left(-p_{2}^{2}\right)^{-1-z_{5}-z_{6}}\left(-p_{3}^{2}\right)^{-1-z_{5}-z_{6}}\left(-p_{4}^{2}\right)^{z_{6}}(-t)^{z_{5}}}{(-s)^{1-z_{5}}} \\
& \times \frac{\Gamma\left(1+z_{3,4}\right) \Gamma\left(1+z_{1}-z_{3,4,5}\right) \Gamma\left(z_{2,3,4,5}-z_{1}\right) \Gamma\left(z_{4}-z_{6}\right)}{\Gamma\left(1+z_{4}-z_{6}\right) \Gamma\left(1+z_{1}-z_{2,3,5,6}\right) \Gamma\left(2+z_{3,5,6}\right) \Gamma\left(2+z_{2,3,5,6}\right)} \prod_{j} \Gamma\left(-z_{j}\right) \\
& \times \Gamma\left(z_{1}-z_{2,3,5,6}\right)^{2} \Gamma\left(1+z_{5,6}\right) \Gamma\left(1+z_{3,5,6}\right) \Gamma\left(1+z_{2,3,5,6}\right)^{2} .
\end{aligned}
$$

Now the simple change of variables $z_{2} \rightarrow-z_{2}+z_{1}-z_{3}-z_{4}-z_{5}$ in (306) leads to an expression identical to (305) up to a factor of $u$ and we obtain the identity $T_{2}^{(0)}=s T_{1}^{(0)}$, which corresponds to the identity $\Phi^{(3)}=\Psi^{(3)}$ of section 2.6.2. (Observe that the factor $s$ here appears because the 
general integrals (303) and (304) are defined without the appropriate prefactors present in the definitions of $\Phi^{(3)}$ and $\Psi^{(3)}$.

Let us stress that one can also apply the technique of MB representation in a similar way in various situations where a given four-point off-shell Feynman integral cannot be reduced to ladder integrals.

\section{B Two-loop calculation of the rectangular light-like Wilson loop}

In this appendix we present the detailed calculation [95, 86] of the two-loop four-point Wilson loop.

\section{B.1 Computation of individual diagrams}

Due to non-Abelian exponentiation, we only need the diagrams containing then non-Abelian colour factor $\sim C_{F} N_{c}$. The two-loop correction $w^{(2)}$ is given by a sum over the individual diagrams shown in Fig. 27 plus crossing symmetric diagrams. It is convenient to expand their contributions in powers of $1 / \epsilon$ and separate the UV divergent and finite parts as follows

$$
w^{(2)}=\sum_{\alpha}\left[\left(-x_{13}^{2} \tilde{\mu}^{2}\right)^{2 \epsilon}+\left(-x_{24}^{2} \tilde{\mu}^{2}\right)^{2 \epsilon}\right]\left\{\frac{1}{\epsilon^{4}} A_{-4}^{(\alpha)}+\frac{1}{\epsilon^{3}} A_{-3}^{(\alpha)}+\frac{1}{\epsilon^{2}} A_{-2}^{(\alpha)}+\frac{1}{\epsilon} A_{-1}^{(\alpha)}\right\}+A_{0}^{(\alpha)}+O(\epsilon),
$$

where the sum goes over the two-loop Feynman diagrams shown in Fig. 27(c)-(l). Here $A_{-n}^{(\alpha)}$ (with $0 \leq n \leq 4$ ) are dimensionless functions of the ratio of distances $x_{13}^{2} / x_{24}^{2}$. Making use of (307), we can parameterise the contribution of each individual diagram to the Wilson loop by the set of coefficient functions $A_{-n}^{(\alpha)}$. In (307) $\tilde{\mu}$ is defined by $\tilde{\mu}^{2}=\mu^{2} e^{\gamma_{\mathrm{E}}} \pi$, where $\gamma_{\mathrm{E}}$ is the Euler constant. The gluon propagator in the Feynman gauge is

$$
D^{\mu \nu}(x)=\eta^{\mu \nu} D(x), \quad D(x)=-\frac{\Gamma(1-\epsilon)}{4 \pi^{2}}\left(e^{-\gamma_{\mathrm{E}}} \tilde{\mu}^{2}\right)^{\epsilon}\left(-x^{2}+i 0\right)^{-1+\epsilon} .
$$

For simplicity we will consider the Wilson loop in the region where $x_{13}^{2}>0, x_{24}^{2}>0$, which allows us to drop the $+i 0$ prescription in the gluon propagator (308). Let us also introduce some notations that will be used throughout this section:

$$
\alpha=x_{13}^{2} /\left(x_{13}^{2}+x_{24}^{2}\right), \quad \bar{\alpha}=1-\alpha, \quad \gamma=\alpha / \bar{\alpha} .
$$

In addition, we will sometimes use the notation

$$
p_{i}^{\mu}=x_{i+1}^{\mu}-x_{i}^{\mu} .
$$

Finally, we will drop a factor of $\left(-\mu^{2}\right)^{2 \epsilon}$ in intermediate steps of the calculation, since it can be easily reinstated at the end.

\section{B.1.1 Contributions independent of the kinematics}

In [85], the two-loop computation was carried out in the special kinematics $x_{13}^{2}=-x_{24}^{2}=1$. Since diagrams $I^{(c)}, I^{(d)}$ and $I^{(f)}$ only depend in the kinematics through trivial prefactors like $\left(-x_{13}^{2} \tilde{\mu}^{2}\right)^{2 \epsilon}$, we can use the results of [85], and find the following contributions 45 to the coefficient

\footnotetext{
${ }^{45}$ For diagram $I^{(c)}$ we take into account the difference between the DREG scheme used in 85] and DRED scheme used here, see [16].
} 
functions $A_{-n}^{(\alpha)}$ defined in (307).

$$
\begin{aligned}
& A_{-4}^{(c)}=0, \quad A_{-3}^{(c)}=\frac{1}{8}, \quad A_{-2}^{(c)}=\frac{1}{4}, \quad A_{-1}^{(c)}=\frac{1}{2}+\frac{\pi^{2}}{48}, \quad A_{0}^{(c)}=2+\frac{\pi^{2}}{12}+\frac{1}{6} \zeta_{3} . \\
& A_{-4}^{(d)}=-\frac{1}{16}, \quad A_{-3}^{(d)}=0, \quad A_{-2}^{(d)}=-\frac{\pi^{2}}{96}, \quad A_{-1}^{(d)}=-\frac{1}{24} \zeta_{3}, \quad A_{0}^{(d)}=-\frac{7}{2880} \pi^{4} . \\
& A_{-4}^{(f)}=\frac{1}{16}, \quad A_{-3}^{(f)}=-\frac{1}{8}, \quad A_{-2}^{(f)}=-\frac{1}{4}+\frac{5}{96} \pi^{2}, \quad A_{-1}^{(f)}=-\frac{1}{2}-\frac{\pi^{2}}{48}+\frac{7}{24} \zeta_{3}, \\
& A_{0}^{(f)}=-2-\frac{\pi^{2}}{12}+\frac{119}{2880} \pi^{4}-\frac{1}{6} \zeta_{3} .
\end{aligned}
$$

Let us now consider the diagrams containing two crossed gluon propagators, i.e. $I^{(e)}, I^{(h)}, I^{(i)}, I^{(j)}$. Their group theory factor is $\operatorname{Tr}\left(t_{a} t_{b} t_{a} t_{b}\right)=C_{F}\left(C_{F}-\frac{1}{2} N_{c}\right)$, of which we keep the part $-\frac{1}{2} C_{F} N_{c}$ only because of non-Abelian exponentiation.

\section{B.1.2 Diagram $I^{(e)}$}

We consider the crossed diagram $\left[p_{1}, p_{4}\right]\left[p_{4}, p_{3}\right]$ (the square brackets indicate the two segments to which the gluon is attached) shown in Fig. 27(e). The other diagrams of the same topology will be taken account of by including a symmetry factor 4 for the contribution to $w^{(2)}$. The contribution of the diagram takes the form

$$
\begin{aligned}
I^{(e)} & =-\frac{1}{2} C_{F} N_{c}(i g)^{4} \int_{0}^{1} d t_{1} \int_{t_{1}}^{1} d t_{2} \int_{0}^{1} d s_{1} \int_{0}^{1} d s_{2} \\
& \times\left(p_{1} \cdot p_{4}\right)\left(p_{3} \cdot p_{4}\right) D\left(p_{1} s_{2}+p_{4} t_{2}\right) D\left(p_{4}\left(1-t_{1}\right)+p_{3} s_{1}\right) .
\end{aligned}
$$

Taking into account the light-likeness of the edges of the Wilson loop are light-like, i.e. $p_{i}^{2}=$ $x_{i, i+1}^{2}=0$, we find

$$
I^{(e)}=-\frac{C_{F} N_{c} g^{4}}{2\left(8 \pi^{2}\right)^{2}} \Gamma^{2}(1-\epsilon)\left(x_{13}^{2} x_{24}^{2} e^{-2 \gamma_{\mathrm{E}}}\right)^{\epsilon} \int_{0}^{1} d t_{1} \int_{t_{1}}^{1} d t_{2} \int_{0}^{1} d s_{1} \int_{0}^{1} d s_{2}\left(s_{2} t_{2}\left(1-t_{1}\right) s_{1}\right)^{-1+\epsilon}
$$

Doing the integrals, one finds that the contribution of the diagram is

$$
I^{(e)}=-\frac{C_{F} N_{c} g^{4}}{2\left(8 \pi^{2}\right)^{2}} \Gamma^{2}(1-\epsilon)\left(x_{13}^{2} x_{24}^{2} e^{-2 \gamma_{\mathrm{E}}}\right)^{\epsilon} \frac{1}{\epsilon^{4}}\left[1-\frac{\Gamma^{2}(1+\epsilon)}{\Gamma(1+2 \epsilon)}\right]
$$

It develops a double pole in $\epsilon$. Including the symmetry factor, its contribution to $w^{(2)}$ is

$$
A_{-2}^{(e)}=-\frac{\pi^{2}}{24}, \quad A_{-1}^{(e)}=\frac{1}{2} \zeta_{3}, \quad A_{0}^{(e)}=-\frac{\pi^{2}}{12} M_{1}-\frac{49}{720} \pi^{4} .
$$

\section{B.1.3 Diagram $I^{(h)}$}

The contribution of this diagram takes the form

$$
\begin{aligned}
I^{(h)} & =-\frac{1}{2} N_{c} C_{F}(i g)^{4} \int_{0}^{1} d t_{1} \int_{t_{1}}^{1} d t_{2} \int_{0}^{1} d s_{1} \int_{0}^{1} d s_{2} \\
& \times\left(p_{1} \cdot p_{4}\right)\left(p_{2} \cdot p_{4}\right) D\left(p_{1} s_{2}+p_{4} t_{2}\right) D\left(p_{1}+p_{2} s_{1}+p_{4} t_{1}\right)
\end{aligned}
$$

Integration over $s_{1}, s_{2}$ and $t_{2}$ yields

$$
\begin{aligned}
I^{(h)} & =\frac{1}{2} N_{c} C_{F} \frac{g^{4}}{\left(8 \pi^{2}\right)^{2}} \Gamma^{2}(1-\epsilon)\left(e^{-2 \gamma_{\mathrm{E}}} x_{24}^{2}\right)^{\epsilon}\left(x_{13}^{2}+x_{24}^{2}\right) \\
& \times \frac{1}{\epsilon^{3}} \int_{0}^{1} \frac{d t_{1}\left(1-t_{1}^{\epsilon}\right)}{x_{13}^{2}-\left(x_{13}^{2}+x_{24}^{2}\right) t_{1}}\left[\left(x_{13}^{2}\left(1-t_{1}\right)\right)^{\epsilon}-\left(x_{24}^{2} t_{1}\right)^{\epsilon}\right]
\end{aligned}
$$


This expression in not symmetric under $\alpha \rightarrow 1-\alpha$. Its symmetrisation gives (upon substitution $x \rightarrow 1-x)$

$$
\begin{aligned}
I^{(h)(\mathrm{sym})=} & \frac{1}{2} C_{F} N_{c} \frac{g^{4}}{\left(8 \pi^{2}\right)^{2}}\left\{\frac{1}{4 \epsilon}\left[\left(x_{13}^{2}\right)^{2 \epsilon}+\left(x_{24}^{2}\right)^{2 \epsilon}\right] \int_{0}^{1} \frac{d x}{x-\alpha} \ln \left(\frac{\alpha \bar{x}}{\bar{\alpha} x}\right) \ln (x \bar{x})\right. \\
& \left.+\frac{1}{4} \int_{0}^{1} \frac{d x}{x-\alpha} \ln \left(\frac{\alpha \bar{x}}{\bar{\alpha} x}\right)\left[\frac{3}{2} \ln ^{2}(x \bar{x})-\frac{1}{2} \ln ^{2}\left(\frac{\bar{\alpha}}{\alpha}\right)+\frac{1}{2} \ln ^{2}\left(\frac{\alpha \bar{x}}{\bar{\alpha} x}\right)\right]+O(\epsilon)\right\},
\end{aligned}
$$

and in the notation of the basic integrals of section B.3

$$
\begin{aligned}
I^{(h)(\mathrm{sym})}= & \frac{1}{8} C_{F} N_{c}\left(\frac{g^{2}}{8 \pi^{2}}\right)^{2}\left\{\frac{1}{\epsilon}\left[\left(x_{13}^{2}\right)^{2 \epsilon}+\left(x_{24}^{2}\right)^{2 \epsilon}\right] M_{2}\right. \\
& \left.+\left[\frac{3}{2} M_{3}-\frac{1}{2} \ln ^{2}\left(\frac{\bar{\alpha}}{\alpha}\right) M_{1}+\frac{1}{2} M_{9}\right]+O(\epsilon)\right\} .
\end{aligned}
$$

Including the combinatorial factor corresponding to this diagram, $c^{(h)}=8$, we have

$$
A_{-1}^{(h)}=\frac{1}{4} M_{2}, \quad A_{0}^{(h)}=\frac{1}{8} M_{1}^{2}+\frac{3}{8} M_{3}+\frac{\pi^{2}}{8} M_{1} .
$$

\section{B.1.4 Diagram $I^{(i)}$}

The contribution of this diagram takes the form

$$
\begin{aligned}
I^{(i)} & =-\frac{1}{2} N_{c} C_{F}(i g)^{4} \int_{0}^{1} d t_{1} \int_{t_{1}}^{1} d t_{2} \int_{0}^{1} d s_{2} \int_{s_{2}}^{1} d s_{1} \\
& \times\left(p_{2} \cdot p_{4}\right)^{2} D\left(p_{1}+p_{2} s_{1}+p_{4} t_{1}\right) D\left(p_{1}+p_{2} s_{2}+p_{4} t_{2}\right)
\end{aligned}
$$

It is finite for $\epsilon \rightarrow 0$ and, therefore, the calculation can be performed in $D=4$ dimensions

$$
I^{(i)}=-\frac{1}{2} N_{c} C_{F} \frac{g^{4}}{\left(8 \pi^{2}\right)^{2}} J
$$

with

$$
J=\int_{0}^{1} d t_{1} \int_{t_{1}}^{1} d t_{2} \int_{0}^{1} d s_{2} \int_{s_{2}}^{1} d s_{1}\left[\left(\alpha s_{1}+\bar{\alpha} t_{1}-s_{1} t_{1}\right)\left(\alpha s_{2}+\bar{\alpha} t_{2}-s_{2} t_{2}\right)\right]^{-1}
$$

Then, after integrating over $s_{2}$ and $t_{1}$ and rescaling $s_{1} \rightarrow \bar{\alpha} s_{1}$ and $t_{2} \rightarrow \alpha t_{2}$ one gets

$$
J=\int_{0}^{1 / \alpha} \int_{0}^{1 / \bar{\alpha}} \frac{d s_{1} d t_{2}}{\left(1-s_{1}\right)\left(1-t_{2}\right)} \ln \left(1+s_{1} \frac{1-t_{2}}{t_{2}}\right) \ln \left(1+t_{2} \frac{1-s_{1}}{s_{1}}\right)
$$

It seems natural to simplify the integrand by changing integration variables

$$
x=s_{1} \frac{1-t_{2}}{t_{2}}, \quad y=t_{2} \frac{1-s_{1}}{s_{1}},
$$

which leads to

$$
J=\int_{\Sigma} d x d y f(x, y), \quad f(x, y)=\frac{1-x y}{x(1+x) y(1+y)} \ln (1+x) \ln (1+y)
$$

where the integration domain $\Sigma$ is defined by

$$
0 \leq \frac{1-x y}{1+y} \leq 1+\frac{1}{\gamma}, \quad 0 \leq \frac{1-x y}{1+x} \leq 1+\gamma, \quad \gamma=\frac{\alpha}{\bar{\alpha}}=\frac{s}{t}>0 .
$$


This can be written more conveniently as

$$
\begin{aligned}
y & \leq \frac{1}{x}, \quad(x>0) \\
y & \leq-\left(1+\gamma+\frac{\gamma}{x}\right), \quad(-1<x<0) \\
y & \geq-\frac{1}{1+\gamma+\gamma x} .
\end{aligned}
$$

Thus the integration over $\Sigma$ can be parametrised as follows:

$$
J=\underbrace{\int_{0}^{\infty} d x \int_{0}^{1 / x} d y f(x, y)}_{J_{1}}+\underbrace{\int_{0}^{\infty} d x \int_{-\frac{1}{1+\gamma+\gamma x}}^{0} d y f(x, y)}_{J_{2}}+\underbrace{\int_{-1}^{0} d x \int_{\frac{-1}{1+\gamma+\gamma x}}^{-(1+\gamma+\gamma / x)} d y f(x, y)}_{J_{34}}
$$

The integration regions lie in the first, second and third and fourth quadrant (clockwise). The first integration can be easily done using

$$
\int^{y} d z f(x, z)=\frac{\ln (1+x)}{x(1+x)}\left[-\frac{1}{2}(1+x) \ln ^{2}(1+y)-\operatorname{Li}_{2}(-y)\right] .
$$

The final integration is harder, but all integrals can be expressed in terms of polylogarithms [221]. After some algebra we find

$$
\begin{aligned}
J_{1}= & \frac{\pi^{4}}{180} \\
J_{2}= & \frac{19 \pi^{4}}{360}+\frac{\pi^{2}}{12} \ln ^{2}(\gamma)+\frac{1}{24} \ln ^{4}(\gamma)+\frac{\pi^{2}}{12} \ln ^{2}(1+\gamma)+\frac{1}{6} \ln (\gamma) \ln ^{3}(1+\gamma)-\frac{1}{12} \ln ^{4}(1+\gamma) \\
& -\left[-\frac{\pi^{2}}{3}-\frac{1}{2} \ln ^{2}(\gamma)+\frac{1}{2} \operatorname{Li}_{2}(-\gamma)\right] \operatorname{Li}_{2}(-\gamma) \\
& -\ln \left(\frac{\gamma}{1+\gamma}\right)\left[\operatorname{Li}_{3}(-\gamma)+\zeta_{3}\right]-\operatorname{Li}_{4}\left(\frac{1}{1+\gamma}\right)-\operatorname{Li}_{4}\left(\frac{\gamma}{1+\gamma}\right) \\
J_{34}= & -\frac{\pi^{4}}{60}-\frac{\pi^{2}}{12} \ln ^{2}(1+\gamma)-\frac{1}{6} \ln (\gamma) \ln ^{3}(1+\gamma)+\frac{1}{12} \ln ^{4}(1+\gamma) \\
& +\left[-\frac{\pi^{2}}{3}-\frac{1}{2} \ln ^{2}(\gamma)+\frac{1}{2} \operatorname{Li}_{2}(-\gamma)\right] \operatorname{Li}_{2}(-\gamma) \\
& +\ln \left(\frac{\gamma}{1+\gamma}\right)\left[\operatorname{Li}_{3}(-\gamma)+\zeta_{3}\right]+\operatorname{Li}_{4}\left(\frac{1}{1+\gamma}\right)+\operatorname{Li}_{4}\left(\frac{\gamma}{1+\gamma}\right)
\end{aligned}
$$

Here we used frequently polylogarithm identities like those given in appendix B.4 in order to simplify the individual expressions. Summing them up, we see that most terms cancel between $J_{2}$ and $J_{34}$, and we obtain the simple result

$$
J=\frac{1}{24}\left[\pi^{2}+\ln ^{2}(\gamma)\right]^{2} .
$$

Including the symmetry factor 2 , we find

$$
A_{0}^{(i)}=-\frac{1}{24} M_{1}^{2} .
$$

\section{B.1.5 Factorised diagram $I^{(j)}$}

The contribution of this diagram takes the form

$$
\begin{aligned}
I^{(j)} & =-\frac{1}{2} C_{F} N_{c}(i g)^{4} \int_{0}^{1} d t_{1} \int_{0}^{1} d t_{2} \int_{0}^{1} d s_{2} \int_{0}^{1} d s_{1} \\
& \times\left(p_{2} \cdot p_{4}\right)\left(p_{1} \cdot p_{3}\right) D\left(p_{2}+p_{1} s_{1}+p_{3} t_{1}\right) D\left(p_{1}+p_{2} s_{2}+p_{4} t_{2}\right)
\end{aligned}
$$


and the integral factorises into the product of finite one-loop integrals,

$$
I^{(j)}=-\frac{1}{2} N_{c} C_{F}\left(\frac{g^{2}}{4 \pi^{2}}\right)^{2}\left[\frac{1}{2} \int_{0}^{1} \frac{d x d y\left(x_{13}^{2}+x_{24}^{2}\right)}{x_{24}^{2} x+x_{13}^{2} y-\left(x_{13}^{2}+x_{24}^{2}\right) x y}\right]^{2} .
$$

Evaluating the integral we find

$$
A_{0}^{(j)}=-\frac{1}{8} M_{1}^{2}
$$

\section{B.1.6 Self-energy diagram $I^{(k)}$}

The contribution of this diagram reads

$$
I^{(k)}=-g^{2} C_{F}\left(p_{2} \cdot p_{4}\right) \int_{0}^{1} d s_{1} \int_{0}^{1} d t_{1} D^{(1)}\left(p_{1}+p_{2} s_{1}+p_{4} t_{1}\right),
$$

where the gluon propagator in the Feynman gauge, with the one-loop correction included, is

$$
D^{(1)}(x)=\frac{g^{2}}{64 \pi^{D}}\left[(3 D-2-2 \epsilon) N_{c}-2(D-2) n_{f}-n_{s}\right] \frac{\Gamma^{2}(D / 2-1)}{(D-4)(D-3)(D-1)}\left(-x^{2}+i 0\right)^{3-D}
$$

with $D=4-2 \epsilon$. Here we added the contribution both of $\epsilon$-scalars in the DRED scheme and of $n_{s}$ scalars. Then,

$$
I^{(k)}=\frac{g^{4}}{2 \cdot 64 \pi^{D}} C_{F}\left[(3 D-2-2 \epsilon) N_{c}-2(D-2) n_{f}-n_{s}\right] \frac{\Gamma^{2}(D / 2-1)}{(D-4)(D-3)(D-1)} J^{(k)},
$$

with

$$
J^{(k)}=\left(x_{13}^{2}+x_{24}^{2}\right) \int_{0}^{1} d s_{1} \int_{0}^{1} d t_{1}\left[-x_{13}^{2} s_{1}-x_{24}^{2} t_{1}+\left(x_{13}^{2}+x_{24}^{2}\right) s_{1} t_{1}\right]^{3-D}
$$

The integration is straightforward. In $\mathcal{N}=4 \mathrm{SYM}$, for $n_{f}=4 N_{c}$ and $n_{s}=6 N_{c}$,

$$
I^{(k)}=\frac{1}{8}\left(\frac{g^{2}}{4 \pi^{2}}\right)^{2} C_{F} N_{c}\left\{\epsilon^{-1}\left[\left(x_{13}^{2}\right)^{2 \epsilon}+\left(x_{24}^{2}\right)^{2 \epsilon}\right] M_{1}+4 M_{1}+2 M_{2}+O(\epsilon)\right\} .
$$

The combinatorial factor corresponding to this diagram is $c^{(k)}=2$, so we have

$$
A_{-1}^{(k)}=\frac{1}{4} M_{1}, \quad A_{0}^{(k)}=M_{1}+\frac{1}{2} M_{2} .
$$

\section{B.1.7 'Mercedes-Benz' diagram $I^{(l)}$}

We assume that two gluon legs are attached to the segment $p_{2}$ and the third leg is attached to the segment $p_{4}$

$$
\begin{aligned}
I^{(l)}= & \frac{1}{2} g^{4} C_{F} N_{c} \int_{0}^{1} d t_{1} \int_{0}^{1} d t_{2} \int_{t_{2}}^{1} d t_{3} \\
& \times p_{4}^{\mu_{1}} p_{2}^{\mu_{2}} p_{2}^{\mu_{3}} \Gamma_{\mu_{1} \mu_{2} \mu_{3}}\left(-i \partial_{z_{1}},-i \partial_{z_{2}},-i \partial_{z_{3}}\right) \int d^{D} z_{4} \prod_{i=1}^{3} D\left(z_{i}-z_{4}\right),
\end{aligned}
$$

where

$$
z_{1}^{\mu}=-p_{1}^{\mu}-p_{4}^{\mu} t_{1}, \quad z_{2}^{\mu}=p_{2}^{\mu} t_{2}, \quad z_{3}^{\mu}=p_{2}^{\mu} t_{3},
$$

and the three-gluon vertex is given by

$$
p_{4}^{\mu_{1}} p_{2}^{\mu_{2}} p_{2}^{\mu_{3}} \Gamma_{\mu_{1} \mu_{2} \mu_{3}}\left(-i \partial_{z_{1}},-i \partial_{z_{2}},-i \partial_{z_{3}}\right)=i\left(p_{2} \cdot p_{4}\right)\left(p_{2} \partial_{z_{2}}-p_{2} \partial_{z_{3}}\right)=i\left(p_{2} \cdot p_{4}\right)\left(\partial_{t_{2}}-\partial_{t_{3}}\right) .
$$


Then,

$$
I^{(l)}=\frac{i}{2} g^{4} C_{F} N\left(p_{2} \cdot p_{4}\right) \int_{0}^{1} d t_{1} \int_{0}^{1} d t_{2} \int_{t_{2}}^{1} d t_{3}\left(\partial_{t_{2}}-\partial_{t_{3}}\right) J\left(z_{1}, z_{2}, z_{3}\right),
$$

where the three-point integral $J\left(z_{1}, z_{2}, z_{3}\right)$ is defined in appendix B.2. By virtue of $z_{23}^{2}=0$

$$
J\left(z_{1}, z_{2}, z_{3}\right)=\frac{i^{1-2 D}}{32 \pi^{D}} \frac{\Gamma(D-3)}{4-D} \int_{0}^{1} d \tau(\tau \bar{\tau})^{D / 2-2}\left[\left(-z_{1}+\tau z_{2}+\bar{\tau} z_{3}\right)^{2}\right]^{3-D}
$$

Using the explicit expression for the $z$-coordinates in (346) and equation (349) we find

$$
\begin{aligned}
I^{(l)} & =i^{2-2 D} \frac{g^{4} C_{F} N}{128 \pi^{D}} \frac{\Gamma(D-3)}{(4-D)^{2}} \int_{0}^{1} \frac{d t_{1}\left(-x_{13}^{2}-x_{24}^{2}\right)}{x_{13}^{2} \bar{t}_{1}-x_{24}^{2} t_{1}} \int_{0}^{1} d \tau(\tau \bar{\tau})^{D / 2-2} \\
& \times\left\{\left(2-\frac{1}{\tau}\right)\left[\left(x_{13}^{2} \bar{t}_{1}\right)^{4-D}-\left(x_{24}^{2} t_{1}\right)^{4-D}\right]+\left(\frac{1}{\tau}-\frac{1}{\bar{\tau}}\right)\left(x_{13}^{2} \bar{\tau}_{1}\right)^{4-D}\right\} .
\end{aligned}
$$

Notice that in the second term, one can integrate by parts using

$$
(\tau \bar{\tau})^{D / 2-2}\left(\frac{1}{\tau}-\frac{1}{\bar{\tau}}\right)=(D / 2-2)^{-1} \frac{d}{d \tau}(\tau \bar{\tau})^{D / 2-2} .
$$

After some algebra one arrives at

$$
\begin{aligned}
I^{(l)} & =\frac{g^{4} C_{F} N}{64 \pi^{4}} \frac{\Gamma^{2}(1-\epsilon)}{(2 \epsilon)^{3}(1-2 \epsilon)} e^{-2 \epsilon \gamma_{\mathrm{E}}} \\
& \times \int_{0}^{1} \frac{d t}{\alpha-t}\left[\left(-x_{13}^{2}\right)^{2 \epsilon}(1-t)^{2 \epsilon}-\left(-x_{24}^{2}\right)^{2 \epsilon} t^{2 \epsilon}\right]\left[1-\frac{\Gamma(2-2 \epsilon)}{\Gamma^{2}(1-\epsilon)}(t(1-t))^{-\epsilon}\right],
\end{aligned}
$$

where we replaced $D$ by $4-2 \epsilon$. Expanding in powers of $\epsilon$ we find

$$
I^{(l)}=-\frac{g^{4} C_{F} N}{64 \pi^{4}}\left\{\frac{1}{8 \epsilon}\left[\left(x_{13}^{2}\right)^{2 \epsilon}+\left(x_{24}^{2}\right)^{2 \epsilon}\right]\left(2 M_{1}+M_{2}\right)+\left(1-\frac{1}{24} \pi^{2}\right) M_{1}+\frac{1}{2} M_{2}+\frac{1}{8} M_{3}+O(\epsilon)\right\},
$$

where we used the basic integrals of section B.3. The combinatorial factor corresponding to this diagram is $c^{(l)}=4$, so we have

$$
A_{-1}^{(l)}=-\frac{1}{4} M_{1}-\frac{1}{8} M_{2}, \quad A_{0}^{(l)}=-M_{1}+\frac{\pi^{2}}{24} M_{1}-\frac{1}{2} M_{2}-\frac{1}{8} M_{3} .
$$

\section{B.1.8 T-shaped diagram}

We assume that two gluon legs are attached to the segment $p_{2}$ and the third leg is attached to the segment $p_{4}$

$$
\begin{aligned}
I^{(g)}= & \frac{1}{2} g^{4} c_{F} N_{c} \int_{0}^{1} d t_{1} \int_{0}^{1} d t_{2} \int_{0}^{1} d t_{3} \\
& \times p_{4}^{\mu_{1}} p_{1}^{\mu_{2}} p_{2}^{\mu_{3}} \Gamma_{\mu_{1} \mu_{2} \mu_{3}}\left(-i \partial_{z_{1}},-i \partial_{z_{2}},-i \partial_{z_{3}}\right) \int d^{D} z_{4} \prod_{i=1}^{3} D\left(z_{i}-z_{4}\right),
\end{aligned}
$$

where

$$
z_{1}=-p_{4} t_{1}, \quad z_{2}=p_{1} t_{2}, \quad z_{3}=p_{1}+p_{2} t_{3}
$$

and three-gluon vertex is given by

$$
\begin{aligned}
\Gamma_{3} & \equiv p_{4}^{\mu_{1}} p_{1}^{\mu_{2}} p_{2}^{\mu_{3}} \Gamma_{\mu_{1} \mu_{2} \mu_{3}}\left(-i \partial_{z_{1}},-i \partial_{z_{2}},-i \partial_{z_{3}}\right) \\
& =i\left(p_{1} \cdot p_{4}\right)\left(p_{2} \cdot\left(\partial_{z_{2}}-\partial_{z_{1}}\right)\right)+i\left(p_{1} \cdot p_{2}\right)\left(p_{4} \cdot\left(\partial_{z_{3}}-\partial_{z_{2}}\right)\right)+i\left(p_{2} \cdot p_{4}\right)\left(p_{1} \cdot\left(\partial_{z_{1}}-\partial_{z_{3}}\right)\right) .
\end{aligned}
$$


Translation invariance of the $z_{4}$-integral can be used to simplify the vertex to

$\Gamma_{3}=-i\left(p_{1} \cdot p_{4}\right) \partial_{t_{3}}-i\left(p_{1} \cdot p_{2}\right) \partial_{t_{1}}-2 i\left(p_{1} \cdot p_{4}\right)\left(p_{2} \cdot \partial_{z_{1}}\right)+2 i\left(p_{1} \cdot p_{2}\right)\left(p_{4} \cdot \partial_{z_{3}}\right)+i\left(p_{2} \cdot p_{4}\right)\left(p_{1} \cdot\left(\partial_{z_{1}}-\partial_{z_{3}}\right)\right)$

Let us rewrite the integral as $I^{(g)}=I_{1}^{(g)}+I_{2}^{(g)}$, where

$$
\begin{aligned}
& I_{1}^{(g)}=\frac{i}{2} g^{4} c_{F} N_{c} \int_{0}^{1} d t_{1} \int_{0}^{1} d t_{2} \int_{0}^{1} d t_{3}\left[\frac{1}{2} x_{24}^{2} \partial_{t_{3}}+\frac{1}{2} x_{13}^{2} \partial_{t_{1}}\right] V\left(z_{1}, z_{2}, z_{3}\right) \\
& I_{2}^{(g)}=\frac{i}{2} g^{4} c_{F} N_{c} \int_{0}^{1} d t_{1} \int_{0}^{1} d t_{2} \int_{0}^{1} d t_{3} \\
& \times\left[-x_{24}^{2}\left(\left(p_{2} \partial_{z_{1}}\right)+\partial_{t_{3}}\right)+x_{13}^{2}\left(\left(p_{4} \cdot \partial_{z_{3}}\right)-\partial_{t_{1}}\right)-\frac{1}{2}\left(x_{13}^{2}+x_{24}^{2}\right)\left(p_{1} \cdot\left(\partial_{z_{1}}-\partial_{z_{3}}\right)\right)\right] V\left(z_{1}, z_{2}, z_{3}\right)
\end{aligned}
$$

The first contribution $I_{1}^{(g)}$ can be split into a divergent and a finite part, $I_{1}^{(g)}=I_{1 d}^{(g)}+I_{1 f}^{(g)}$

$$
\begin{aligned}
I_{1 d}^{(g)} & =\frac{i}{2} g^{4} c_{F} N_{c} \int_{0}^{1} d t_{2}\left[-\frac{1}{2} x_{13}^{2} \int_{0}^{1} d t_{3} V\left(0, z_{2}, z_{3}\right)-\frac{1}{2} x_{24}^{2} \int_{0}^{1} d t_{1} V\left(z_{1}, z_{2}, p_{1}\right)\right], \\
I_{1 f}^{(g)} & =\frac{i}{2} g^{4} c_{F} N_{c} \int_{0}^{1} d t_{2}\left[+\frac{1}{2} x_{13}^{2} \int_{0}^{1} d t_{3} V\left(-p_{4}, z_{2}, z_{3}\right)+\frac{1}{2} x_{24}^{2} \int_{0}^{1} d t_{1} V\left(z_{1}, z_{2}, p_{1}+p_{2}\right)\right] .
\end{aligned}
$$

Divergences come from the two integrals in the first line only since the three arguments of the $V$-functions in second line cannot be light-like. The divergent integrals can be easily evaluated as

$$
I_{1 d}^{(g)}=\left(\frac{g^{2}}{32 \pi^{2}}\right)^{2} C_{F} N \frac{\Gamma^{2}(1-\epsilon) e^{-2 \epsilon \gamma_{\mathrm{E}}}}{\epsilon^{4}}\left[\left(x_{13}^{2}\right)^{2 \epsilon}+\left(x_{24}^{2}\right)^{2 \epsilon}\right]\left[1-\frac{\Gamma(1+\epsilon) \Gamma(1-2 \epsilon)}{\Gamma(1-\epsilon)}\right] .
$$

Next, we examine the finite part of $I_{1}$. it can be written as

$$
I_{1 f}^{(g)}=\frac{g^{4} C_{F} N_{c}}{4 \cdot 64 \pi^{4}} \int[d \beta]_{3} \int_{0}^{1} d t_{2} \int_{0}^{1} d t_{1} \frac{x_{24}^{2}}{\beta_{3}\left(\beta_{2} \bar{t}_{2}+\beta_{1} \bar{t}_{1}\right) x_{13}^{2}+\beta_{1} t_{1} \beta_{2} t_{2} x_{24}^{2}}+\left[x_{14}^{2} \leftrightarrows x_{13}^{2}\right]
$$

Here $[d \beta]_{3}=d \beta_{1} d \beta_{2} d \beta_{3} \delta\left(1-\beta_{1}-\beta_{2}-\beta_{3}\right)$. After changing variables, we have

$$
I_{1 f}^{(g)}=\frac{g^{4} C_{F} N_{c}}{4 \cdot 64 \pi^{4}} J_{1 f}^{(g)},
$$

where

$$
J_{1 f}^{(g)}=\int_{0}^{1} d x \int_{0}^{1} d \lambda \int_{0}^{1} d t_{2} \int_{0}^{1} d t_{1} \frac{1}{\bar{\lambda}\left(x \bar{t}_{2}+\bar{x} \bar{t}_{1}\right) \gamma+\lambda x \bar{x} t_{1} t_{2}}+(\gamma \rightarrow 1 / \gamma)
$$

After some algebra one finds

$$
\begin{aligned}
J_{1 f}^{(g)}= & \left\{\frac{7}{60} \pi^{4}+\frac{\pi^{2}}{3} \ln ^{2}(\gamma)+\frac{1}{12} \ln ^{4}(\gamma)-\frac{2}{3} \pi^{2} \ln (\gamma) \ln (1+\gamma)-\frac{1}{3} \ln ^{3}(\gamma) \ln (1+\gamma)\right. \\
& +\frac{2}{3} \pi^{2} \ln ^{2}(1+\gamma)+\frac{1}{2} \ln ^{2}(\gamma) \ln ^{2}(1+\gamma)-\frac{1}{6} \ln ^{4}(1+\gamma)+\frac{\pi^{2}}{3} \operatorname{Li}_{2}(-\gamma) \\
& \left.+2 \ln (\gamma) \operatorname{Li}_{3}\left(\frac{\gamma}{1+\gamma}\right)-2 \ln (\gamma) \zeta_{3}+2 \operatorname{Li}_{4}(-\gamma)-2 \operatorname{Li}_{4}\left(\frac{1}{1+\gamma}\right)-2 \operatorname{Li}_{4}\left(\frac{\gamma}{1+\gamma}\right)\right\} \\
& +(\gamma \rightarrow 1 / \gamma) .
\end{aligned}
$$

Let us now study $I_{2}^{(g)}$. For $D=4-2 \epsilon$ it is given by

$$
\begin{aligned}
I_{2}^{(g)} & =-\frac{g^{4} C_{F} N_{c}}{4 \cdot 64 \pi^{4}} J_{2}^{(g)}, \\
J_{2}^{(g)} & =-(1-2 \epsilon) \Gamma(1-2 \epsilon) \int_{0}^{1} d t_{1} \int_{0}^{1} d t_{2} \int_{0}^{1} d t_{3} \int_{0}^{1}[d \beta]_{3}\left(\beta_{1} \beta_{2} \beta_{3}\right)^{-\epsilon} \\
& \times \frac{\left(x_{13}^{2}+x_{24}^{2}\right)\left[\beta_{1} t_{1}\left(\beta_{2}-2 \beta_{3}\right) x_{24}^{2}+\beta_{3} t_{3}\left(\beta_{2}-2 \beta_{1}\right) x_{13}^{2}\right]}{\left[\beta_{3} t_{3}\left(\beta_{2} \bar{t}_{2}+\beta_{1} \bar{t}_{1}\right) x_{13}^{2}+\beta_{1} t_{1}\left(\beta_{2} t_{2}+\beta_{3} \bar{t}_{3}\right) x_{24}^{2}\right]^{2-2 \epsilon} .}
\end{aligned}
$$


Then, we change the integration variables according to

$$
\beta_{1}=\lambda x, \quad \beta_{2}=\bar{\lambda}, \quad \beta_{3}=\lambda \bar{x},
$$

and integrate over $t_{2}$, which leads to

$$
\begin{aligned}
J_{2}^{(g)} & =\left(x_{13}^{2}\right)^{2 \epsilon}(1+\gamma) \int_{0}^{1} d t_{1} d t_{3} \int_{0}^{1} d \lambda \bar{\lambda}^{-1-\epsilon} \int_{0}^{1} d x(x \bar{x})^{-\epsilon} \frac{\bar{x} t_{3}(\bar{\lambda}-2 \lambda x)+x t_{1}(\bar{\lambda}-2 \lambda \bar{x}) \gamma}{x t_{1} \gamma-\bar{x} t_{3}} \\
& \times\left\{x^{-1+2 \epsilon}\left[\bar{x} t_{3} \lambda \bar{t}_{1}+t_{1}\left(\bar{\lambda}+\lambda \bar{x} \bar{t}_{3}\right) \gamma\right]^{-1+2 \epsilon}-\bar{x}^{-1+2 \epsilon}\left[t_{3}\left(\bar{\lambda}+\lambda x \bar{t}_{1}\right)+x t_{1} \lambda \bar{t}_{3} \gamma\right]^{-1+2 \epsilon}\right\}
\end{aligned}
$$

We notice that integration over small $x$ and $\bar{x}$ produces a single pole in $\epsilon$. We split up $J_{2}^{(g)}$ into a simplified divergent integral and several convergent integrals, in which we neglect $O(\epsilon)$ terms.

$$
\begin{aligned}
J_{2 d}^{(g)}= & \left(x_{13}^{2}\right)^{2 \epsilon}(1+\gamma) \int_{0}^{1} d t_{1} d t_{3} \int_{0}^{1} d \lambda \bar{\lambda}^{-\epsilon} \int_{0}^{1} d x \\
\times & \left\{-x^{-1+\epsilon}\left[t_{3} \lambda \bar{t}_{1}+t_{1}\left(\bar{\lambda}+\lambda \bar{t}_{3}\right) \gamma\right]^{-1+2 \epsilon}-\bar{x}^{-1+\epsilon}\left[t_{3}\left(\bar{\lambda}+\lambda \bar{t}_{1}\right)+t_{1} \lambda \bar{t}_{3} \gamma\right]^{-1+2 \epsilon}\right\} \\
J_{2 f}^{(g)}= & (1+\gamma) \int_{0}^{1} d t_{1} d t_{3} \int_{0}^{1} d \lambda \int_{0}^{1} d x \\
\times & \left\{-2 \frac{\lambda}{\bar{\lambda}} \frac{t_{3}+t_{1} \gamma}{x t_{1} \gamma-\bar{x} t_{3}}\left[\frac{x}{\bar{x} t_{3} \lambda \bar{t}_{1}+t_{1}\left(\bar{\lambda}+\lambda \bar{x} \bar{t}_{3}\right) \gamma}-\frac{t_{3}\left(\bar{\lambda}+\lambda x \bar{t}_{1}\right)+x t_{1} \lambda \bar{t}_{3} \gamma}{t_{3}}\right]\right. \\
& +2 \frac{1}{x t_{1} \gamma-\bar{x} t_{3}}\left[\frac{t_{3}}{\bar{x} t_{3} \lambda \bar{t}_{1}+t_{1}\left(\bar{\lambda}+\lambda \bar{x} \bar{t}_{3}\right) \gamma}-\frac{1}{t_{3}\left(\bar{\lambda}+\lambda x \bar{t}_{1}\right)+x t_{1} \lambda \bar{t}_{3} \gamma}\right] \\
& -\frac{1}{x}\left[\frac{1}{\bar{x} t_{3} \lambda \bar{t}_{1}+t_{1}\left(\bar{\lambda}+\lambda \bar{x} \bar{t}_{3}\right) \gamma}-\frac{1}{t_{3} \lambda \bar{t}_{1}+t_{1}\left(\bar{\lambda} \lambda \bar{t}_{3}\right) \gamma}\right] \\
& \left.-\frac{1}{\bar{x}}\left[\frac{1}{t_{3}\left(\bar{\lambda}+\lambda x \bar{t}_{1}\right)+x t_{1} \lambda \bar{t}_{3} \gamma}-\frac{1}{t_{3}\left(\bar{\lambda}+\lambda \bar{t}_{1}\right)+t_{1} \lambda \bar{t}_{3} \gamma}\right]\right\}
\end{aligned}
$$

To summarize,

$$
J_{2}^{(g)}=J_{2 d}^{(g)}+J_{2 f}^{(g)}+O(\epsilon)
$$

where only the first term produces poles in $\epsilon$. Calculating $J_{2 d}^{(g)}$ we get

$$
\begin{aligned}
J_{2 d}^{(g)}= & \frac{1}{\epsilon}\left(x_{13}^{2}+x_{24}^{2}\right)^{2 \epsilon} \int_{0}^{1} \frac{d x}{x-\alpha} \ln \frac{x \bar{\alpha}}{\bar{x} \alpha} \ln (x \bar{x}) \\
& -\int_{0}^{1} \frac{d x}{x-\alpha}\left[\ln (x \bar{x})\left(\ln ^{2}(\bar{x} \alpha)-\ln ^{2}(x \bar{\alpha})\right)+\ln \frac{x \bar{\alpha}}{\bar{x} \alpha}\left(\frac{\pi^{2}}{6}+\ln x \ln \bar{x}\right)\right],
\end{aligned}
$$

or equivalently, writing $J_{2 d}^{(g)}=J_{2 d d}^{(g)}+J_{2 d f}^{(g)}$,

$$
\begin{aligned}
J_{2 d d}^{(g)} & =\frac{1}{2 \epsilon}\left[\left(x_{13}^{2}\right)^{2 \epsilon}+\left(x_{24}^{2}\right)^{2 \epsilon}\right] M_{2} \\
J_{2 d f}^{(g)} & =-\ln (\alpha \bar{\alpha}) M_{2}-\int_{0}^{1} \frac{d x}{x-\alpha}\left[\ln (x \bar{x})\left(\ln ^{2}(\bar{x} \alpha)-\ln ^{2}(x \bar{\alpha})\right)+\ln \frac{x \bar{\alpha}}{\bar{x} \alpha}\left(\frac{\pi^{2}}{6}+\ln x \ln \bar{x}\right)\right]
\end{aligned}
$$

where the integral $M_{2}$ is defined in (377). From (356) and (362) we can read off the contribution of $I^{(g)}$ to the pole coefficients (taking into account the combinatorial factor 4 for this diagram),

$$
A_{-2}^{(g)}=\frac{\pi^{2}}{48}, \quad A_{-1}^{(g)}=-\frac{1}{8} M_{2}+\frac{1}{8} \zeta_{3} .
$$


Let us now turn to the finite part. All integrals appearing in it can be evaluated in terms of polylogarithms. After a lot of algebra, one finds

$$
\begin{aligned}
J_{2 d f}^{(g)}= & \left\{\frac{11}{60} \pi^{4}+\frac{\pi^{2}}{4} \ln ^{2}(\gamma)+\frac{1}{12} \ln ^{4}(\gamma)-\frac{3}{2} \pi^{2} \ln (\gamma) \ln (1+\gamma)-\frac{1}{2} \ln ^{3}(\gamma) \ln (1+\gamma)\right. \\
& +2 \pi^{2} \ln ^{2}(1+\gamma)+\frac{3}{2} \ln ^{2}(\gamma) \ln ^{2}(1+\gamma)-\frac{1}{2} \ln ^{4}(1+\gamma)+3 \ln (\gamma) \operatorname{Li}_{3}(-\gamma) \\
& \left.6 \ln (\gamma) \operatorname{Li}_{3}\left(\frac{\gamma}{1+\gamma}\right)-3 \ln (\gamma) \zeta_{3}-6 \operatorname{Li}_{4}\left(\frac{1}{1+\gamma}\right)-6 \operatorname{Li}_{4}\left(\frac{\gamma}{1+\gamma}\right)\right\}
\end{aligned}
$$

and

$$
\begin{aligned}
J_{2 f}^{(g)}= & \left\{\frac{47}{120} \pi^{2}+\frac{\pi^{2}}{2} \ln ^{2}(\gamma)+\frac{1}{8} \ln ^{4}(\gamma)-\frac{3}{2} \pi^{2} \ln (\gamma) \ln (1+\gamma)-\frac{1}{2} \ln ^{3}(\gamma) \ln (1+\gamma)\right. \\
& +2 \pi^{2} \ln ^{2}(1+\gamma)+\frac{3}{2} \ln ^{2}(\gamma) \ln ^{2}(1+\gamma)-\frac{1}{2} \ln ^{4}(1+\gamma)+3 \ln (\gamma) \operatorname{Li}_{3}(-\gamma) \\
& \left.+6 \ln (\gamma) \operatorname{Li}_{3}\left(\frac{\gamma}{1+\gamma}\right)-3 \ln (\gamma) \zeta_{3}-6 \operatorname{Li}_{4}\left(\frac{1}{1+\gamma}\right)-6 \operatorname{Li}_{4}\left(\frac{\gamma}{1+\gamma}\right)\right\}
\end{aligned}
$$

Summing up (360), (365) and (366) we find

$$
-J_{1 f}^{(g)}+J_{2 d f}^{(g)}+J_{2 f}^{(g)}=\frac{1}{6} M_{1}^{2}+\frac{11}{90} \pi^{4}-M_{3} .
$$

Thus, taking into account the symmetry factor 4 and a constant contribution coming from equation (362), we find the total contribution to the finite part of this diagram to be

$$
A_{0}^{(g)}=\frac{1}{24} M_{1}^{2}-\frac{1}{4} M_{3}+\frac{7}{360} \pi^{4} .
$$

This completes the calculation. Summing up all contributions, we find formula (307).

\section{B.2 Useful formulae for diagrams with three-gluon vertex}

In configuration space, these diagrams involve three propagators joined at the same point $z$ which is integrated out

$$
V\left(z_{1}, z_{2}, z_{3}\right)=\mu^{-2 \epsilon} \int d^{D} z D\left(z-z_{1}\right) D\left(z-z_{2}\right) D\left(z-z_{3}\right)
$$

with $D=4-2 \epsilon$ and $D(z)$ being the gluon propagator in the Feynman gauge

$$
D(z)=-\frac{i^{-1+\epsilon}}{4 \pi^{2}}\left(\pi \mu^{2}\right)^{\epsilon} \int_{0}^{\infty} d s s^{-\epsilon} \mathrm{e}^{-i s z^{2}} .
$$

Shifting variable $z \rightarrow z+z_{1}$ and performing the integration one gets

$$
V\left(z_{1}, z_{2}, z_{3}\right)=\frac{i^{2 \epsilon}}{64 \pi^{4}}\left(\pi \mu^{2}\right)^{2 \epsilon} \int_{0}^{\infty} d s_{1} d s_{2} d s_{3} \frac{\left(s_{1} s_{2} s_{3}\right)^{-\epsilon}}{\left(s_{1}+s_{2}+s_{3}\right)^{2-\epsilon}} \exp \left(i A\left(s_{1}, s_{2}, s_{3}\right)\right),
$$

with

$$
A\left(s_{1}, s_{2}, s_{3}\right)=\frac{\left(s_{2} z_{21}+s_{3} z_{31}\right)^{2}}{s_{1}+s_{2}+s_{3}}-s_{2} z_{21}^{2}-s_{3} z_{31}^{2}=-\frac{s_{1} s_{2} z_{12}^{2}+s_{2} s_{3} z_{23}^{2}+s_{3} s_{1} z_{31}^{2}}{s_{1}+s_{2}+s_{3}} .
$$

Changing integration variables according to $s_{i}=\lambda \beta_{i}$ with $\sum_{i} \beta_{i}=1$ and $0 \leq \beta_{i} \leq 1$, one obtains

$$
V\left(z_{1}, z_{2}, z_{3}\right)=-\frac{i^{1+4 \epsilon}}{64 \pi^{4}}\left(\pi \mu^{2}\right)^{2 \epsilon} \Gamma(1-2 \epsilon) \int_{0}^{1} \frac{[d \beta]_{3}\left(\beta_{1} \beta_{2} \beta_{3}\right)^{-\epsilon}}{\left[\beta_{1} \beta_{2} z_{12}^{2}+\beta_{2} \beta_{3} z_{23}^{2}+\beta_{3} \beta_{1} z_{31}^{2}\right]^{1-2 \epsilon}} .
$$


Let us consider the integral in the kinematics $\left(z_{2}-z_{3}\right)^{2}=0$, where it can be further simplified. In that case,

$$
A\left(s_{1}, s_{2}, s_{3}\right)=-\frac{s_{1}\left(s_{2}+s_{3}\right)}{s_{1}+s_{2}+s_{3}}\left[z_{1}-\frac{s_{2} z_{2}+s_{3} z_{3}}{s_{2}+s_{3}}\right]^{2}
$$

Then, we introduce standard parametrization

$$
s_{i}=\rho \beta_{i}, \quad \beta_{1}=1-\lambda, \quad \beta_{2}=\lambda x, \quad \beta_{2}=\lambda(1-x)
$$

and find

$$
V\left(z_{1}, z_{2}, z_{3}\right)=-\frac{i^{1+4 \epsilon}}{64 \pi^{4}}\left(\pi \mu^{2}\right)^{2 \epsilon} \frac{\Gamma(1-2 \epsilon)}{\epsilon} \int_{0}^{1} \frac{d x(x \bar{x})^{-\epsilon}}{\left[\left(x z_{21}+\bar{x} z_{31}\right)^{2}\right]^{1-2 \epsilon}}
$$

\section{B.3 Basic integrals}

Here we give explicit expressions for integrals encountered in the two-loop calculation.

$$
\begin{aligned}
M_{1}= & \int_{0}^{1} \frac{d x}{x-\bar{\alpha}} \ln \left(\frac{\bar{\alpha} \bar{x}}{\alpha x}\right)=-\frac{1}{2}\left[\pi^{2}+\ln ^{2}\left(\frac{\alpha}{\bar{\alpha}}\right)\right] \\
M_{2}= & \int_{0}^{1} \frac{d x}{x-\bar{\alpha}} \ln \left(\frac{\bar{\alpha} \bar{x}}{\alpha x}\right) \ln (x \bar{x}) \\
= & -\frac{\pi^{2}}{2} \ln (\alpha \bar{\alpha})+2 \operatorname{Li}_{3}(1)-\operatorname{Li}_{3}\left(-\frac{\alpha}{\bar{\alpha}}\right)-\operatorname{Li}_{3}\left(-\frac{\bar{\alpha}}{\alpha}\right)-\ln \left(\frac{\alpha}{\bar{\alpha}}\right)\left[\operatorname{Li}_{2}(\alpha)-\operatorname{Li}_{2}(\bar{\alpha})\right] \\
M_{3}= & \int_{0}^{1} \frac{d x}{x-\bar{\alpha}} \ln \left(\frac{\bar{\alpha} \bar{x}}{\alpha x}\right) \ln ^{2}(x \bar{x}) \\
= & -\frac{49}{180} \pi^{4}-\frac{1}{3} \pi^{2}\left[\ln ^{2}(\alpha)+6 \ln (\alpha) \ln (\bar{\alpha})+\ln ^{2}(\bar{\alpha})\right] \\
& -\frac{1}{12}\left[\ln ^{4}(\alpha)+\ln ^{4}(\bar{\alpha})+4 \ln (\bar{\alpha}) \ln ^{3}(\alpha)-18 \ln ^{2}(\alpha) \ln ^{2}(\bar{\alpha})+4 \ln (\alpha) \ln ^{3}(\bar{\alpha})\right] \\
& -4 \ln \left(\frac{\alpha}{\bar{\alpha}}\right)\left[\operatorname{Li}_{3}(\alpha)-\operatorname{Li}_{3}(\bar{\alpha})\right]+8\left[\operatorname{Li}_{4}(\alpha)+\operatorname{Li}_{4}(\bar{\alpha})\right] \\
M_{9}= & \int_{0}^{1} \frac{d x}{x-\alpha} \ln ^{3}\left(\frac{\alpha \bar{x}}{\bar{\alpha} x}\right)=-\frac{1}{4}\left(\ln ^{2} \frac{\alpha}{\bar{\alpha}}+\pi^{2}\right)^{2}=-M_{1}^{2},
\end{aligned}
$$

where $\bar{\alpha}=1-\alpha$ and $\operatorname{Li}_{n}(z)$ (with $n=2,3,4$ ) are polylogarithms [221].

\section{B.4 Identities for polylogarithms of related arguments}

Most (or all) of the following polylogarithm identities can be found in Lewin's book [221]. They can be easily proved by differentiating (the integration constant can be fixed by evaluating the identity for a particular value of $z$ ).

$$
\begin{gathered}
\operatorname{Li}_{2}(-z)+\operatorname{Li}_{2}\left(-\frac{1}{z}\right)=-\frac{1}{2} \ln ^{2}(z)-\frac{\pi^{2}}{6}, \quad z>0 . \\
\operatorname{Li}_{2}(z)+\mathrm{Li}_{2}(1-z)=-\ln (z) \ln (1-z)+\frac{\pi^{2}}{6}, \quad 0<z<1 .
\end{gathered}
$$

It follows from (382), by putting $z \rightarrow 1 /(1+z)$,

$$
\operatorname{Li}_{2}\left(\frac{1}{1+z}\right)+\operatorname{Li}_{2}\left(\frac{z}{1+z}\right)=-\ln \left(\frac{1}{1+z}\right) \ln \left(\frac{z}{1+z}\right)+\frac{\pi^{2}}{6}, \quad z>0 .
$$

Similar useful identities are

$$
\operatorname{Li}_{2}(1-z)+\operatorname{Li}_{2}\left(1-\frac{1}{z}\right)=-\frac{1}{2} \ln ^{2}(z), \quad z>0 .
$$


and

$$
\operatorname{Li}_{2}(-z)+\operatorname{Li}_{2}\left(\frac{z}{1+z}\right)=-\frac{1}{2} \ln ^{2}(1+z), \quad z>0 .
$$

Using (381), (383) and (385) the functions $\mathrm{Li}_{2}\left(-\frac{1}{z}\right), \mathrm{Li}_{2}\left(\frac{1}{1+z}\right), \mathrm{Li}_{2}\left(\frac{z}{1+z}\right)$ can all be reexpressed in terms of $\mathrm{Li}_{2}(-z)$ and logarithms. The inversion identity for $\mathrm{Li}_{3}$ reads

$$
\mathrm{Li}_{3}(-z)-\mathrm{Li}_{3}\left(-\frac{1}{z}\right)=-\frac{\pi^{2}}{6} \ln (z)-\frac{1}{6} \ln ^{3}(z), \quad z>0 .
$$

There is also

$$
\begin{aligned}
& \operatorname{Li}_{3}(-z)+\operatorname{Li}_{3}\left(\frac{1}{1+z}\right)+\operatorname{Li}_{3}\left(\frac{z}{1+z}\right)-\zeta_{3} \\
= & \frac{1}{3} \ln ^{3}(1+z)-\frac{1}{2} \ln (z) \ln ^{2}(1+z)-\frac{\pi^{2}}{6} \ln (1+z), \quad z>0 .
\end{aligned}
$$

For $\mathrm{Li}_{4}$ there is just the inversion identity:

$$
\mathrm{Li}_{4}(-z)+\mathrm{Li}_{4}\left(-\frac{1}{z}\right)=-\frac{\pi^{2}}{12} \ln ^{2}(z)-\frac{1}{24} \ln ^{4}(z)-\frac{7 \pi^{4}}{360}, \quad z>0 .
$$

\section{References}

[1] G. 't Hooft, Nucl. Phys. B72 (1974) 461.

[2] L.N. Lipatov, Phys. Lett. B309 (1993) 394.

[3] L.D. Faddeev and G.P. Korchemsky, Phys. Lett. B342 (1995) 311, hep-th/9404173.

[4] J.M. Maldacena, Adv. Theor. Math. Phys. 2 (1998) 231, hep-th/9711200.

[5] A.V. Belitsky, Phys. Lett. B453 (1999) 59, hep-ph/9902361.

[6] A.V. Belitsky, Nucl. Phys. B558 (1999) 259, hep-ph/9903512.

[7] A.V. Belitsky, Nucl. Phys. B574 (2000) 407, hep-ph/9907420.

[8] V.M. Braun, S.E. Derkachov and A.N. Manashov, Phys. Rev. Lett. 81 (1998) 2020, hep-ph/9805225.

[9] V.M. Braun et al., Nucl. Phys. B553 (1999) 355, hep-ph/9902375.

[10] S.E. Derkachov, G.P. Korchemsky and A.N. Manashov, Nucl. Phys. B566 (2000) 203, hep-ph/9909539.

[11] J.A. Minahan and K. Zarembo, JHEP 03 (2003) 013, hep-th/0212208.

[12] N. Beisert et al., Phys. Lett. B558 (2003) 229, hep-th/0212269.

[13] N. Beisert, C. Kristjansen and M. Staudacher, Nucl. Phys. B664 (2003) 131, hep-th/0303060.

[14] N. Beisert and M. Staudacher, Nucl. Phys. B670 (2003) 439, hep-th/0307042.

[15] N. Beisert, Nucl. Phys. B682 (2004) 487, hep-th/0310252.

[16] A.V. Belitsky, A.S. Gorsky and G.P. Korchemsky, Nucl. Phys. B667 (2003) 3, hep-th/0304028. 
[17] N. Beisert, Nucl. Phys. B676 (2004) 3, hep-th/0307015.

[18] N. Beisert, JHEP 09 (2003) 062, hep-th/0308074.

[19] L. Dolan, C.R. Nappi and E. Witten, JHEP 10 (2003) 017, hep-th/0308089.

[20] G. Arutyunov and M. Staudacher, JHEP 03 (2004) 004, hep-th/0310182.

[21] L. Dolan, C.R. Nappi and E. Witten, (2004), hep-th/0401243.

[22] A.V. Belitsky et al., Phys. Lett. B594 (2004) 385, hep-th/0403085.

[23] A.V. Belitsky et al., Nucl. Phys. B708 (2005) 115, hep-th/0409120.

[24] A.V. Ryzhov and A.A. Tseytlin, Nucl. Phys. B698 (2004) 132, hep-th/0404215.

[25] S.A. Frolov, R. Roiban and A.A. Tseytlin, JHEP 07 (2005) 045, hep-th/0503192.

[26] R.R. Metsaev and A.A. Tseytlin, Nucl. Phys. B533 (1998) 109, hep-th/9805028.

[27] I. Bena, J. Polchinski and R. Roiban, Phys. Rev. D69 (2004) 046002, hep-th/0305116.

[28] B.C. Vallilo, JHEP 03 (2004) 037, hep-th/0307018.

[29] N. Berkovits, JHEP 03 (2005) 041, hep-th/0411170.

[30] N. Beisert, B. Eden and M. Staudacher, J. Stat. Mech. 0701 (2007) P021, hep-th/0610251.

[31] M. Staudacher, JHEP 05 (2005) 054, hep-th/0412188.

[32] G. Arutyunov, S. Frolov and M. Staudacher, JHEP 10 (2004) 016, hep-th/0406256.

[33] N. Beisert and M. Staudacher, Nucl. Phys. B727 (2005) 1, hep-th/0504190.

[34] R.A. Janik, Phys. Rev. D73 (2006) 086006, hep-th/0603038.

[35] N. Beisert, R. Hernandez and E. Lopez, JHEP 11 (2006) 070, hep-th/0609044.

[36] A.M. Polyakov, Nucl. Phys. B164 (1980) 171.

[37] G.P. Korchemsky and A.V. Radyushkin, Phys. Lett. B171 (1986) 459.

[38] G.P. Korchemsky and A.V. Radyushkin, Nucl. Phys. B283 (1987) 342.

[39] Z. Bern, L.J. Dixon and V.A. Smirnov, Phys. Rev. D72 (2005) 085001, hep-th/0505205.

[40] Z. Bern et al., Phys. Rev. D75 (2007) 085010, hep-th/0610248.

[41] S.S. Gubser, I.R. Klebanov and A.M. Polyakov, Nucl. Phys. B636 (2002) 99, hep-th/0204051.

[42] S. Frolov and A.A. Tseytlin, JHEP 06 (2002) 007, hep-th/0204226.

[43] R. Roiban and A.A. Tseytlin, JHEP 11 (2007) 016, 0709.0681.

[44] M.K. Benna et al., Phys. Rev. Lett. 98 (2007) 131603, hep-th/0611135.

[45] B. Basso, G.P. Korchemsky and J. Kotanski, Phys. Rev. Lett. 100 (2008) 091601, 0708.3933.

[46] I. Kostov, D. Serban and D. Volin, JHEP 08 (2008) 101, 0801.2542. 
[47] S.J. Parke and T.R. Taylor, Phys. Rev. Lett. 56 (1986) 2459.

[48] F. Berends and W. Giele, Nucl. Phys. B306 (1988) 759.

[49] R. Britto, F. Cachazo and B. Feng, Nucl. Phys. B715 (2005) 499, hep-th/0412308.

[50] R. Britto et al., Phys. Rev. Lett. 94 (2005) 181602, hep-th/0501052.

[51] E. Witten, Commun. Math. Phys. 252 (2004) 189, hep-th/0312171.

[52] Z. Bern et al., Nucl. Phys. B425 (1994) 217, hep-ph/9403226.

[53] Z. Bern et al., Nucl. Phys. B435 (1995) 59, hep-ph/9409265.

[54] R.E. Cutkosky, J. Math. Phys. 1 (1960) 429.

[55] Z. Bern, J.S. Rozowsky and B. Yan, Phys. Lett. B401 (1997) 273, hep-ph/9702424.

[56] C. Anastasiou et al., Phys. Rev. Lett. 91 (2003) 251602, hep-th/0309040.

[57] Z. Bern, L.J. Dixon and D.A. Kosower, Annals Phys. 322 (2007) 1587, 0704.2798.

[58] R. Akhoury, Phys. Rev. D19 (1979) 1250.

[59] A.H. Mueller, Phys. Rev. D20 (1979) 2037.

[60] J.C. Collins, Phys. Rev. D22 (1980) 1478.

[61] J.C. Collins, D.E. Soper and G. Sterman, Adv. Ser. Direct. High Energy Phys. 5 (1988) 1, hep-ph/0409313.

[62] J.C. Collins, Adv. Ser. Direct. High Energy Phys. 5 (1989) 573, hep-ph/0312336.

[63] A. Sen, Phys. Rev. D24 (1981) 3281.

[64] A. Sen, Phys. Rev. D28 (1983) 860.

[65] G. Sterman, Nucl. Phys. B281 (1987) 310.

[66] S. Catani and L. Trentadue, Nucl. Phys. B327 (1989) 323.

[67] S. Catani and L. Trentadue, Nucl. Phys. B353 (1991) 183.

[68] L. Magnea and G. Sterman, Phys. Rev. D42 (1990) 4222.

[69] S. Catani, Phys. Lett. B427 (1998) 161, hep-ph/9802439.

[70] G. Sterman and M.E. Tejeda-Yeomans, Phys. Lett. B552 (2003) 48, hep-ph/0210130.

[71] J. Botts and G. Sterman, Nucl. Phys. B325 (1989) 62.

[72] G.P. Korchemsky, Phys. Lett. B220 (1989) 629.

[73] G.P. Korchemsky, Phys. Lett. B217 (1989) 330.

[74] G.P. Korchemsky and G. Marchesini, Phys. Lett. B313 (1993) 433.

[75] I.A. Korchemskaya and G.P. Korchemsky, Phys. Lett. B387 (1996) 346, hep-ph/9607229.

[76] Z. Bern et al., Phys. Rev. Lett. 97 (2006) 181601, hep-th/0604074.

[77] J.M. Drummond et al., JHEP 01 (2007) 064, hep-th/0607160. 
[78] Z. Bern et al., Phys. Rev. D76 (2007) 125020, 0705.1864.

[79] L.F. Alday and J.M. Maldacena, JHEP 06 (2007) 064, 0705.0303.

[80] L.F. Alday and J.M. Maldacena, JHEP 11 (2007) 068, 0710.1060.

[81] J.M. Maldacena, Phys. Rev. Lett. 80 (1998) 4859, hep-th/9803002.

[82] M. Kruczenski, JHEP 12 (2002) 024, hep-th/0210115.

[83] J.M. Drummond, G.P. Korchemsky and E. Sokatchev, Nucl. Phys. B795 (2008) 385, 0707.0243.

[84] A. Brandhuber, P. Heslop and G. Travaglini, Nucl. Phys. B794 (2008) 231, 0707.1153.

[85] I.A. Korchemskaya and G.P. Korchemsky, Phys. Lett. B287 (1992) 169.

[86] J.M. Drummond et al., Nucl. Phys. B795 (2008) 52, 0709.2368.

[87] J.M. Drummond et al., (2007), 0712.1223.

[88] F. Alday and J. Maldacena, (2007), unpublished.

[89] Z. Komargodski, JHEP 05 (2008) 019, 0801.3274.

[90] J.M. Drummond et al., Phys. Lett. B662 (2008) 456, 0712.4138.

[91] J.M. Drummond et al., (2008), 0803.1466.

[92] Z. Bern et al., Phys. Rev. D78 (2008) 045007, 0803.1465.

[93] R.C. Brower et al., (2008), 0801.3891.

[94] J. Bartels, L.N. Lipatov and A.S. Vera, (2008), 0802.2065.

[95] J.M. Drummond et al., (2007), unpublished.

[96] I.T. Todorov, M.C. Mintchev and V.B. Petkova, Pisa, Italy: Sc. Norm. Sup. (1978) 273p.

[97] E.S. Fradkin and M.Y. Palchik, Phys. Rept. 44 (1978) 249.

[98] V.M. Braun, G.P. Korchemsky and D. Mueller, Prog. Part. Nucl. Phys. 51 (2003) 311, hep-ph/0306057.

[99] F. Gliozzi, J. Scherk and D. Olive, Nucl. Phys. B122 (1977) 253.

[100] L. Brink, J.H. Schwarz and J. Scherk, Nucl. Phys. B121 (1977) 77.

[101] P. Di Francesco, P. Mathieu and D. Senechal, New York, USA: Springer (1997) 890 p.

[102] J.C. Collins, Cambridge, Uk: Univ. Pr. (1984) 380p.

[103] D.I. Kazakov, Phys. Lett. B133 (1983) 406.

[104] Y.M. Makeenko, Sov. J. Nucl. Phys. 33 (1981) 440.

[105] J. Henn, C. Jarczak and E. Sokatchev, Nucl. Phys. B730 (2005) 191, hep-th/0507241.

[106] E.J. Schreier, Phys. Rev. D3 (1971) 980.

[107] J. Erdmenger and H. Osborn, Nucl. Phys. B483 (1997) 431, hep-th/9605009. 
[108] G.M. Sotkov and R.P. Zaikov, Rept. Math. Phys. 12 (1977) 375.

[109] G.M. Sotkov and R.P. Zaikov, Rept. Math. Phys. 19 (1984) 335.

[110] J. Wess and J. Bagger, Princeton, USA: Univ. Pr. (1992) 259 p.

[111] D. Bailin and A. Love, Bristol, UK: IOP (1994) 322 p. (Graduate student series in physics).

[112] M.F. Sohnius, Phys. Rept. 128 (1985) 39.

[113] J.H. Park, Nucl. Phys. B559 (1999) 455, hep-th/9903230.

[114] M. Grisaru, M. Rocek and W. Siegel, Phys. Rev. Lett. 45 (1980) 1063.

[115] W.E. Caswell and D. Zanon, Phys. Lett. B100 (1981) 152.

[116] L.V. Avdeev and O.V. Tarasov, Phys. Lett. B112 (1982) 356.

[117] P.S. Howe, K.S. Stelle and P.K. Townsend, Nucl. Phys. B214 (1983) 519.

[118] S. Mandelstam, Nucl. Phys. B213 (1983) 149.

[119] L. Brink, O. Lindgren and B.E.W. Nilsson, Phys. Lett. B123 (1983) 323.

[120] P.S. Howe, K.S. Stelle and P.C. West, Phys. Lett. B124 (1983) 55.

[121] P.S. Howe, K.S. Stelle and P.K. Townsend, Nucl. Phys. B236 (1984) 125.

[122] A.S. Galperin et al., Cambridge, UK: Univ. Pr. (2001) 306 p.

[123] B. Eden et al., Nucl. Phys. B607 (2001) 191, hep-th/0009106.

[124] B. Eden, Nucl. Phys. B738 (2006) 409, hep-th/0501234.

[125] F. Delduc and J. McCabe, Class. Quant. Grav. 6 (1989) 233.

[126] M. Bianchi et al., Nucl. Phys. B584 (2000) 216, hep-th/0003203.

[127] L. Andrianopoli et al., Adv. Theor. Math. Phys. 4 (2000) 1149, hep-th/9912007.

[128] M. Bianchi, Nucl. Phys. Proc. Suppl. 102 (2001) 56, hep-th/0103112.

[129] T.E. Clark, O. Piguet and K. Sibold, Nucl. Phys. B143 (1978) 445.

[130] K. Konishi, Phys. Lett. B135 (1984) 439.

[131] T. Ohrndorf, Nucl. Phys. B198 (1982) 26.

[132] N.S. Craigie, V.K. Dobrev and I.T. Todorov, Ann. Phys. 159 (1985) 411.

[133] S.J. Brodsky et al., Phys. Rev. D33 (1986) 1881.

[134] D. Mueller, Phys. Rev. D49 (1994) 2525.

[135] A.V. Belitsky et al., Phys. Rev. D77 (2008) 045029, 0707.2936.

[136] A.V. Kotikov et al., Phys. Lett. B595 (2004) 521, hep-th/0404092.

[137] B. Eden, C. Schubert and E. Sokatchev, Phys. Lett. B482 (2000) 309, hep-th/0003096.

[138] G. Arutyunov, S. Frolov and A. Petkou, Nucl. Phys. B586 (2000) 547, hep-th/0005182. 
[139] F.A. Dolan and H. Osborn, Annals Phys. 321 (2006) 581, hep-th/0412335.

[140] S. Penati, A. Santambrogio and D. Zanon, Nucl. Phys. B593 (2001) 651, hep-th/0005223.

[141] D.J. Broadhurst, Phys. Lett. B307 (1993) 132.

[142] G. 't Hooft and M.J.G. Veltman, Nucl. Phys. B153 (1979) 365.

[143] N.I. Usyukina and A.I. Davydychev, Phys. Lett. B298 (1993) 363.

[144] N.I. Usyukina and A.I. Davydychev, Phys. Lett. B305 (1993) 136.

[145] G. Duplancic and B. Nizic, Eur. Phys. J. C24 (2002) 385, hep-ph/0201306.

[146] Z. Bern et al., (2008), 0803.0494.

[147] H. Kawai, D.C. Lewellen and S.H.H. Tye, Nucl. Phys. B269 (1986) 1.

[148] Z. Bern, Living Rev. Rel. 5 (2002) 5, gr-qc/0206071.

[149] Z. Bern et al., Phys. Rev. Lett. 98 (2007) 161303, hep-th/0702112.

[150] Z. Bern and D.A. Kosower, Nucl. Phys. B379 (1992) 451.

[151] Z. Bern et al., Phys. Rev. D66 (2002) 085002, hep-ph/0202271.

[152] K. Risager, (2008), 0804.3310.

[153] L.J. Dixon, (1996), hep-ph/9601359.

[154] M.T. Grisaru, H.N. Pendleton and P. van Nieuwenhuizen, Phys. Rev. D15 (1977) 996.

[155] M.T. Grisaru and H.N. Pendleton, Nucl. Phys. B124 (1977) 81.

[156] N. Kidonakis, G. Oderda and G. Sterman, Nucl. Phys. B525 (1998) 299, hep-ph/9801268.

[157] N. Kidonakis, G. Oderda and G. Sterman, Nucl. Phys. B531 (1998) 365, hep-ph/9803241.

[158] G.P. Korchemsky, Mod. Phys. Lett. A4 (1989) 1257.

[159] G.P. Korchemsky and G. Marchesini, Nucl. Phys. B406 (1993) 225, hep-ph/9210281.

[160] S. Catani and M. Grazzini, Nucl. Phys. B570 (2000) 287, hep-ph/9908523.

[161] F. Cachazo, M. Spradlin and A. Volovich, Phys. Rev. D76 (2007) 106004, 0707.1903.

[162] G. Passarino and M.J.G. Veltman, Nucl. Phys. B160 (1979) 151.

[163] R.J. Eden et al., Cambridge University Press .

[164] R. Britto, F. Cachazo and B. Feng, Nucl. Phys. B725 (2005) 275, hep-th/0412103.

[165] F. Cachazo, M. Spradlin and A. Volovich, Phys. Rev. D74 (2006) 045020, hep-th/0602228.

[166] M. Nakanishi, Routledge; 1 edition (January 1, 1971) .

[167] F. Cachazo, (2008), 0803.1988.

[168] D. Nguyen, M. Spradlin and A. Volovich, Phys. Rev. D77 (2008) 025018, 0709.4665.

[169] F. Cachazo and D. Skinner, (2008), 0801.4574. 
[170] D.J. Gross and P.F. Mende, Phys. Lett. B197 (1987) 129.

[171] J. McGreevy and A. Sever, JHEP 02 (2008) 015, 0710.0393.

[172] S. Ryang, JHEP 05 (2008) 021, 0803.3855.

[173] M. Kruczenski et al., Nucl. Phys. B791 (2008) 93, 0707.4254.

[174] N. Drukker, D. Gross and H. Ooguri, Phys. Rev. D60 (1999) 125006, hep-th/9904191.

[175] K.G. Wilson, Phys. Rev. D10 (1974) 2445.

[176] V.S. Dotsenko and S.N. Vergeles, Nucl. Phys. B169 (1980) 527.

[177] J.L. Gervais and A. Neveu, Nucl. Phys. B163 (1980) 189.

[178] R.A. Brandt, F. Neri and M.A. Sato, Phys. Rev. D24 (1981) 879.

[179] I.Y. Arefeva, Phys. Lett. B93 (1980) 347.

[180] R.A. Brandt et al., Phys. Rev. D26 (1982) 3611.

[181] I.A. Korchemskaya and G.P. Korchemsky, Nucl. Phys. B437 (1995) 127, hep-ph/9409446.

[182] S.J. Rey, S. Theisen and J.T. Yee, Nucl. Phys. B527 (1998) 171, hep-th/9803135.

[183] J. Erickson, G. Semenoff and K. Zarembo, Nucl. Phys. B582 (2000) 155, hep-th/0003055.

[184] G.W. Semenoff and D. Young, Phys. Lett. B643 (2006) 195, hep-th/0609158.

[185] H. Ooguri, Class. Quant. Grav. 17 (2000) 1225, hep-th/9909040.

[186] G.W. Semenoff and K. Zarembo, Nucl. Phys. Proc. Suppl. 108 (2002) 106, hep-th/0202156.

[187] H. Nastase, (2007), 0712.0689.

[188] Y.M. Makeenko and A.A. Migdal, Phys. Lett. B88 (1979) 135.

[189] Y. Makeenko and A.A. Migdal, Nucl. Phys. B188 (1981) 269.

[190] A. Bassetto et al., Nucl. Phys. B408 (1993) 62, hep-ph/9303314.

[191] G. Leibbrandt, Rev. Mod. Phys. 59 (1987) 1067.

[192] R.K. Ellis et al., Nucl. Phys. B152 (1979) 285.

[193] Y.L. Dokshitzer, D. Diakonov and S.I. Troian, Phys. Rept. 58 (1980) 269.

[194] J.G.M. Gatheral, Phys. Lett. B133 (1983) 90.

[195] J. Frenkel and J.C. Taylor, Nucl. Phys. B246 (1984) 231.

[196] E.I. Buchbinder, Phys. Lett. B654 (2007) 46, 0706.2015.

[197] L.F. Alday and J.M. Maldacena, JHEP 11 (2007) 019, 0708.0672.

[198] S.V. Ivanov and G.P. Korchemsky, Phys. Lett. B154 (1985) 197.

[199] S.V. Ivanov, G.P. Korchemsky and A.V. Radyushkin, Yad. Fiz. 44 (1986) 230.

[200] G.P. Korchemsky and A.V. Radyushkin, Phys. Lett. B279 (1992) 359, hep-ph/9203222. 
[201] L.J. Dixon, L. Magnea and G. Sterman, JHEP 08 (2008) 022, 0805.3515.

[202] S. Sarkar, Nucl. Phys. B83 (1974) 108.

[203] V.A. Smirnov, Phys. Lett. B460 (1999) 397, hep-ph/9905323.

[204] J.B. Tausk, Phys. Lett. B469 (1999) 225, hep-ph/9909506.

[205] E.W.N. Glover and M.E. Tejeda-Yeomans, Nucl. Phys. Proc. Suppl. 89 (2000) 196, hep-ph/0010031.

[206] T. Gehrmann and E. Remiddi, Nucl. Phys. Proc. Suppl. 89 (2000) 251, hep-ph/0005232.

[207] C. Anastasiou, J.B. Tausk and M.E. Tejeda-Yeomans, Nucl. Phys. Proc. Suppl. 89 (2000) 262, hep-ph/0005328.

[208] V.A. Smirnov and O.L. Veretin, Nucl. Phys. B566 (2000) 469, hep-ph/9907385.

[209] C. Anastasiou et al., Nucl. Phys. B580 (2000) 577, hep-ph/0003261.

[210] V.A. Smirnov, Phys. Lett. B547 (2002) 239, hep-ph/0209193.

[211] V.A. Smirnov, Phys. Lett. B567 (2003) 193, hep-ph/0305142.

[212] E. Remiddi and J.A.M. Vermaseren, Int. J. Mod. Phys. A15 (2000) 725, hep-ph/9905237.

[213] V.A. Smirnov, Phys. Lett. B491 (2000) 130, hep-ph/0007032.

[214] V.A. Smirnov, Phys. Lett. B500 (2001) 330, hep-ph/0011056.

[215] V.A. Smirnov, Phys. Lett. B524 (2002) 129, hep-ph/0111160.

[216] V.A. Smirnov, Nucl. Phys. Proc. Suppl. 135 (2004) 252, hep-ph/0406052.

[217] G. Heinrich and V.A. Smirnov, Phys. Lett. B598 (2004) 55, hep-ph/0406053.

[218] M. Czakon, J. Gluza and T. Riemann, Acta Phys. Polon. B36 (2005) 3319, hep-ph/0511187.

[219] V.A. Smirnov, Springer Tracts Mod. Phys. 211 (2004) 1.

[220] M. Czakon, Comput. Phys. Commun. 175 (2006) 559, hep-ph/0511200.

[221] L. Lewin, North-Holland, Amsterdam (1981), 359 p. 